Florida International University FIU Digital Commons

\title{
Quantitative Spatial Upscaling of Categorical Data in the Context of Landscape Ecology: A New Scaling Algorithm
}

Daniel Gann

Florida International University, gannd@fiu.edu

DOI: $10.25148 /$ etd.FIDC006878

Follow this and additional works at: https:// digitalcommons.fiu.edu/etd

Part of the Biology Commons, and the Ecology and Evolutionary Biology Commons

\section{Recommended Citation}

Gann, Daniel, "Quantitative Spatial Upscaling of Categorical Data in the Context of Landscape Ecology: A New Scaling Algorithm" (2018). FIU Electronic Theses and Dissertations. 3641.

https://digitalcommons.fiu.edu/etd/3641 


\section{FLORIDA INTERNATIONAL UNIVERSITY}

Miami, Florida

QUANTITATIVE SPATIAL UPSCALING OF CATEGORICAL DATA IN THE CONTEXT OF LANDSCAPE ECOLOGY: A NEW SCALING ALGORITHM

A dissertation submitted in partial fulfillment of

the requirements for the degree of

DOCTOR OF PHILOSOPHY

in

BIOLOGY

by

Daniel Gann

2018 
To: Dean Michael R. Heithaus

College of Arts, Sciences and Education

This dissertation, written by Daniel Gann, and entitled Quantitative Spatial Upscaling of Categorical Data in the Context of Landscape Ecology: A New Scaling Algorithm, having been approved in respect to style and intellectual content, is referred to you for judgment.

We have read this dissertation and recommend that it be approved.

Steve Oberbauer

Leonard Pearlstine

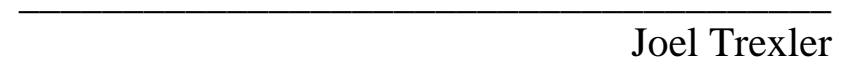

Jennifer H. Richards, Co-Major Professor

Keqi Zhang, Co-Major Professor

Date of Defense: June 28, 2018

The dissertation of Daniel Gann is approved.

Dean Michael R. Heithaus

College of Arts, Sciences and Education

Andrés G. Gil

Vice President for Research and Economic Development and Dean of the University Graduate School

Florida International University, 2018 


\section{ABSTRACT OF THE DISSERTATION \\ QUANTITATIVE SPATIAL UPSCALING OF CATEGORICAL DATA IN \\ THE CONTEXT OF LANDSCAPE ECOLOGY: A NEW SCALING ALGORITHM \\ by}

Daniel Gann

Florida International University, 2018

Miami, Florida

Professor Jennifer H. Richards, Co-Major Professor

Professor Keqi Zhang, Co-Major Professor

Spatially explicit ecological models rely on spatially exhaustive data layers that have scales appropriate to the ecological processes of interest. Such data layers are often categorical raster maps derived from high-resolution, remotely sensed data that must be scaled to a lower spatial resolution to make them compatible with the scale of ecological analysis. Statistical functions commonly used to aggregate categorical data are majority-, nearest-neighbor- and random-rule. For heterogeneous landscapes and large scaling factors, however, use of these functions results in two critical issues: (1) ignoring large portions of information present in the high-resolution grid cells leads to high and uncontrolled loss of information in the scaled dataset; and (2) maintaining classes from the high-resolution dataset at the lower spatial resolution assumes validity of the classification scheme at the low-resolution scale, failing to represent recurring mixes of heterogeneous classes present in the low-resolution grid cells. The proposed new scaling algorithm resolves these issues, aggregating categorical data while simultaneously controlling for 
information loss by generating a non-hierarchical, representative, classification system valid at the aggregated scale.

Implementing scaling parameters, that control class-label precision effectively reduced information loss of scaled landscapes as class-label precision increased. In a neutral-landscape simulation study, the algorithm consistently preserved information at a significantly higher level than the other commonly used algorithms. When applied to maps of real landscapes, the same increase in information retention was observed, and the scaled classes were detectable from lower-resolution, remotely sensed, multi-spectral reflectance data with high accuracy. The framework developed in this research facilitates scalingparameter selection to address trade-offs among information retention, label fidelity, and spectral detectability of scaled classes.

When generating high spatial resolution land-cover maps, quantifying effects of sampling intensity, feature-space dimensionality and classifier method on overall accuracy, confidence estimates, and classifier efficiency allowed optimization of the mapping method. Increase in sampling intensity boosted accuracies in a reasonably predictable fashion. However, adding a second image acquired when ground conditions and vegetation phenology differed from those of the first image had a much greater impact, increasing classification accuracy even at low sampling intensities, to levels not reached with a single season image. 


\section{TABLE OF CONTENTS}

CHAPTER

PAGE

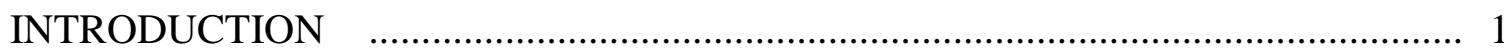

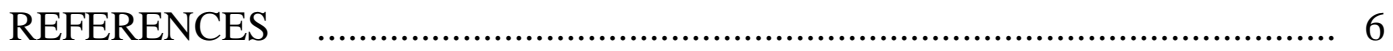

CHAPTER I QUANTITATIVE SPATIAL UPSCALING OF

CATEGORICAL INFORMATION: THE MULTI-DIMENSIONAL

GRID POINT SCALING ALGORITHM _........................................................ 7

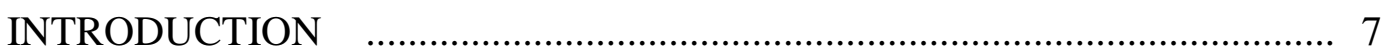

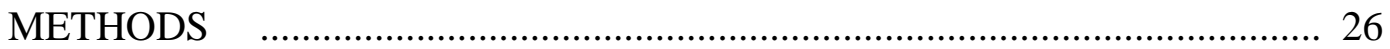

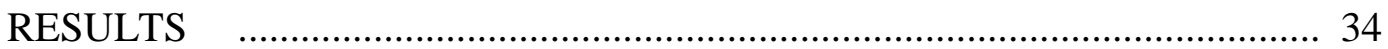

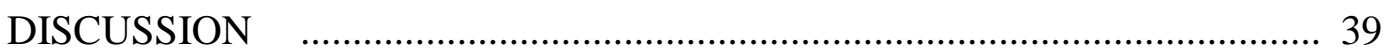

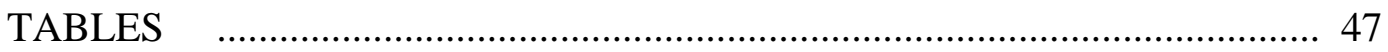

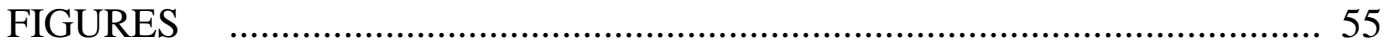

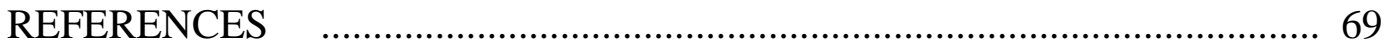

CHAPTER II QUANTITATIVE SPATIAL UPSCALING OF

CLASSIFICATION SYSTEMS USING A MULTI-DIMENSIONAL GRID

POINT CLASSIFIER - REAL LANDSCAPE APPLICATIONS ............................ 76

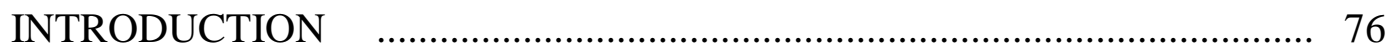

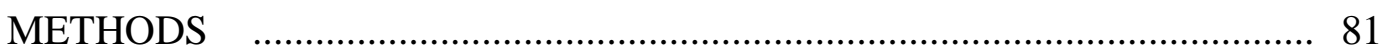

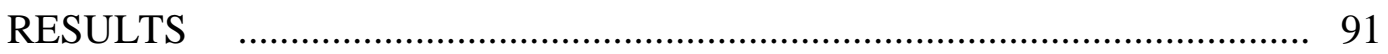

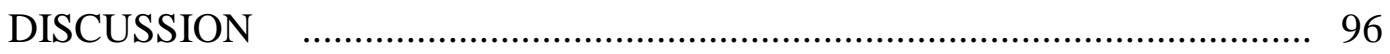

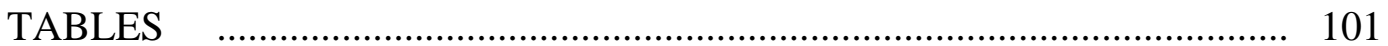

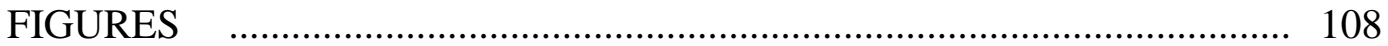

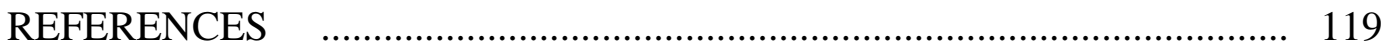

CHAPTER III EFFECTS OF SAMPLING INTENSITY, SEASONALITY

AND CLASSIFIER SELECTION ON CLASSIFICATION

ACCURACY, CONFIDENCE AND EFFICIENCY IN WETLAND

VEGETATION MAPPING

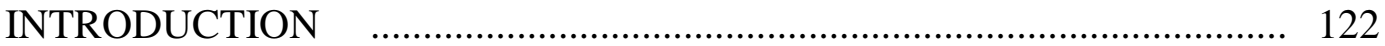

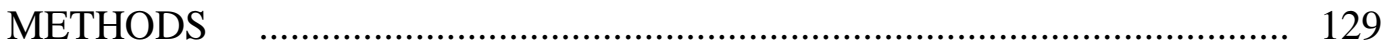

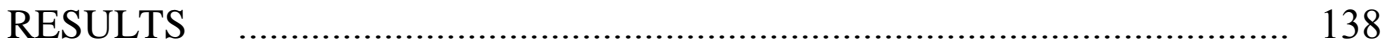

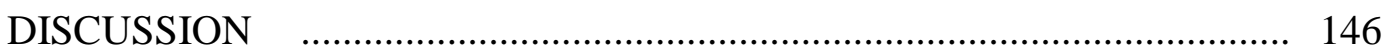

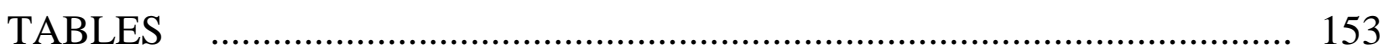

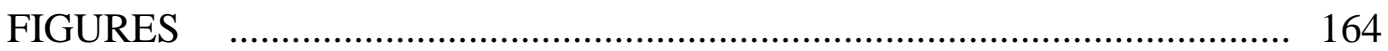

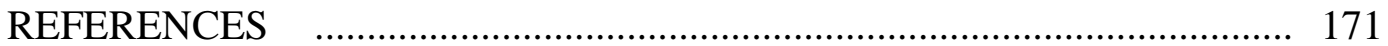

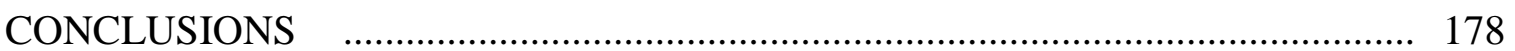

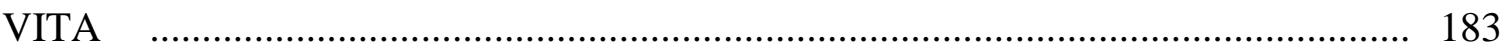




\section{LIST OF TABLES}

TABLE

PAGE

\section{CHAPTER I}

1.1. Number of weak compositions for compositions with constraint of exactly $100 \%$ coverage.

1.2. Number of constrained combinations and precision limits for equal-part partitioning of $\mathrm{n}$ dimensions (richness).

1.3. Mean information retention \pm standard deviations across landscape iterations and random origin for random-rule, majority-rule and MDGP-scaling algorithm with class-label precision of $33 \%$.

1.4. Consistency of the MDGP-scaling algorithm assessed for four levels of classlabel precision and four scale factors.

1.5. Effects of class-label precision and scale factor on information retention for 24 landscape types varying in class-abundance distribution, richness, and spatial aggregation.

1.6. Differences in mean information retention as class-label precision increases for 24 landscape types varying in class-abundance distribution, richness, and spatial aggregation.

1.7. Effects of class-label precision and scale factor on class-label fidelity for 24 landscape types varying in class-abundance distribution, richness, and spatial aggregation.

1.8. Differences in class-label fidelity as class-label precision increases for 24 landscape types varying in class-abundance distribution, richness, and spatial aggregation.

1.9. Optimization of class-label precision parameter for a scale factor of 15 when considering change in information retention, class-label fidelity and in- to output class-count ratio as class-label precision increases.

\section{CHAPTER II}

2.1. Classification scheme of high-resolution plant community map for WCA3A and mapped class proportions for the study area.

2.2. Classification scheme of high-resolution plant community map for NESRS and 
mapped class proportions for the study area.

2.3. WCA3A scaling results for varying class-label precisions and landscape representativeness thresholds.

2.4. Scaling effects on spectral detection accuracies for WCA3A for varying classlabel precisions and landscape representativeness thresholds.

2.5. WCA3A Class schema and class proportions for two good scaling solutions of a 3-part class-label precision with a landscape representativeness of $15 \%$ and a 4-part class-label precision with a representativeness of $15 \%$.

2.6. NESRS scaling results for varying class-label precisions and landscape representativeness thresholds.

2.7. Scaling effects on spectral detection accuracies for NESRS for varying classlabel precisions and landscape representativeness threshold.

2.8. NESRS class schema and class proportions for an optimal scaling solution of a 3-part class-label precision with a landscape representativeness of $10 \%$.

\section{CHAPTER III}

3.1. Classification scheme for the high-resolution plant community maps of the ridge and slough landscape in Water Conservation Area 3A.

3.2. The 72 model sets for each combination of a full factorial design of two classifiers, six variable sets and six sampling intensities $(2 \times 6 \times 6)$.

3.3. Comparison of overall accuracy for naïve Bayes and random forest classifiers by variable set and sampling intensity.

3.4. Comparison of overall accuracy differences for the naïve Bayes classifier when comparing variable sets by sampling intensity.

3.5. Comparison of overall accuracy differences for the random forest classifier when comparing variable sets by sampling intensity.

3.6. Location-specific confidence $\geq 90 \%$ aggregated for all pixels across landscape by variable set and sampling intensity. Increase in confidence diminishes with sampling intensity (bottom)

3.7. Class-specific mean and standard error estimates of producer's and user's accuracies in percent by variable set. 
3.8. Class-specific user's accuracy differences by variable set. Upper and lower $95 \%$ confidence estimates and median difference estimates.

3.9. Class-specific producer's accuracy differences by variable set. Upper and lower $95 \%$ confidence estimates and median difference estimates. 


\section{LIST OF FIGURES}

FIGURE

PAGE

\section{CHAPTER I}

1.1. Two common issues associated with majority-, nearest-neighbor- and randomrule scaling algorithms.

1.2. Ternary plots for three classes and a scale factor of three with 55 possible combinations of the three classes and a scale factor of five with 351 weak combinations.

1.3. Ternary plots of three classes for multi-dimensional grid points of 2, 3, 4 and 5 parts representing $50 \%, 33.3 \%, 25 \%$ and $20 \%$ class-label precisions.

1.4. Schema of framework to test the effects of landscape characteristics, scale factor and class-label precision on information retention, class-count consistency and classlabel fidelity in a full factorial design.

1.5. Neutral landscapes with equal class-abundance distribution of three classes and nine classes for the lowest spatial aggregation factor 0.0 , an aggregation of 0.3 , and the highest aggregation of 1.0.

1.6. Neutral landscapes with geometric class-abundance distribution of three classes and nine classes for the lowest spatial aggregation factor 0.0 , an aggregation of 0.3 , and the highest aggregation of 1.0 .

1.7. Algorithm comparison for mean information retention with increasing scale factor for three scaling algorithms in simulated landscapes with equal classabundance distribution.

1.8. Algorithm comparison of mean information retention with increasing scale factor for three scaling algorithms in simulated landscapes with geometric classabundance distribution.

1.9. Sensitivity of information retention to landscape characteristics and scaling parameters for landscapes with equal class-abundance distribution

1.10. Sensitivity of information retention to landscape characteristics and scaling parameters for landscapes with geometric class-abundance distribution.

1.11. Sensitivity of class-label fidelity evaluated across all landscape iterations with the same characteristics for five random origins for landscapes with equal class-abundance distribution. 
1.12. Sensitivity of class-label fidelity evaluated across all landscape iterations with the same characteristics for five random origins for landscapes with geometric class-abundance distribution.

1.13. Relationships of information retention to class-count ratio and class-label fidelity for landscapes with equal class-abundance distribution and a scale factor of 15.

1.14. Relationships of information retention to class-count ratio and class-label fidelity for landscapes with geometric class-abundance distribution and a scale factor of 15 .

\section{CHAPTER II}

2.1. Study areas in Water Conservation Area 3A and Northeast Shark River Slough within the boundaries of Everglades National Park.

2.2. High-resolution plant communities for WCA3A.

2.3. High-resolution plant communities for NESRS.

2.4. Class-label fidelity and information retention for WCA3A. Mean probability of class-label recurrence across all random-origin scaling results vs. mean landscapelevel information retention.

2.5. Cross-validated overall accuracy for WCA3A. For the same class-label precision, as representativeness increases and small classes are removed, information retention decreases and classification accuracy increases.

2.6. Scaled plant communities for WCA3A and location-specific information retention in percent for the assigned community class label when compared to the high-resolution map.

2.7. Scaled plant-community classes predicted from Landsat spectral data and location-specific classifier probability for class-label assignment for WCA3A.

2.8. Class-label fidelity and information retention for NESRS. Mean probability of class-label recurrence across all random-origin scaling results vs. mean landscapelevel information retention.

2.9. Cross-validated overall accuracy for NESRS. For the same class-label precision, as representativeness increases and small classes are removed, information retention decreases and classification accuracy increases. 
2.10. Scaled plant communities for NESRS and location-specific information retention in percent for the assigned community class label when compared to the high-resolution map.

2.11. Scaled plant-community classes predicted from Landsat spectral data and location-specific classifier probability for class-label assignment for NESRS.

\section{CHAPTER III}

3.1. Study area: Two $1-\mathrm{km}^{2}$ areas located in southern WCA3A and three examples of ridge and slough vegetation types. Aerial photography ( $\sim \mathrm{cm}$ resolution) was acquired by a fixed-wing Unmanned Aerial System in 2012.

3.2. Overall accuracy by sampling intensity and variable set. Diminished returns regression model for random forest classifier and naïve Bayes classifier.

3.3. Training time in seconds as a function of sampling intensity, number of features and number of classes for Naïve Bayes and Random Forest classifiers.

3.4. Prediction times for Naïve Bayes and Random Forest classifiers as a function of optimal number of selected features and number of classes.

3.5. Percentage of map area for which location-specific confidence $\geq 90 \%$.

3.6. Location-specific class membership confidence by sampling intensity for subregion East.

3.7. Class-specific user's and producer's accuracies and 95\% confidence intervals for ridge classes.

3.8. Class-specific user's and producer's accuracies and 95\% confidence intervals for slough classes. 


\section{ABBREVIATIONS AND ACRONYMS}

\begin{tabular}{|c|c|}
\hline Agg. & Aggregation \\
\hline aSpblF & Aquatic Submerged - Periphyton - Broadleaved Floating \\
\hline AVHRR & Advanced Very-High-Resolution Radiometer \\
\hline blFaS & Broadleaved Floating - Aquatic Submerged \\
\hline blFNy & Broadleaved Floating Nymphaea \\
\hline Brdlv. & Broadleaved \\
\hline CAD & Class-Abundance Distribution \\
\hline $\mathrm{CI}$ & Confidence Interval \\
\hline CIL & Lower Confidence Interval Limit \\
\hline CIU & Upper Confidence Interval Limit \\
\hline CLF & Class-Label Fidelity \\
\hline CLFm & Class-Label Fidelity mean \\
\hline CLFp & Class-Label Fidelity proportion \\
\hline clsLS & Class count across landscape \\
\hline clsMn & Class mean \\
\hline clsMono & Monotypic classes \\
\hline clsNum & Number of classes \\
\hline clsRO & Class count for Random Origin \\
\hline clsSD & Class standard deviation \\
\hline clsTot & Total number of classes \\
\hline Conf. & Confidence \\
\hline $\mathrm{cvOA}$ & Cross-validated overall classification accuracy \\
\hline
\end{tabular}




\begin{tabular}{ll} 
Dns. & Dense \\
Emrg. & Emergent \\
Est. Diff. & Estimated Difference \\
Eqs. & Equations \\
FLAASH & Fast Line-of-sight Atmospheric Analysis of Hypercubes \\
Float. & Floating \\
FPAR & Fraction of Photosynthetically Active Radiation \\
GE & Greater Equal \\
GP & Grid Point \\
gMblE & Graminoid Marsh - Broadleaved Emergent \\
gMCl & Graminoid Marsh Cladium \\
gMCID & Graminoid Marsh Cladium Dense \\
gMClS & Graminoid Marsh Cladium Sparse \\
gMD & Graminoid Marsh Dense \\
gMS & Graminoid Marsh Sparse \\
Grm. & Graminoid \\
IR & Information Retention \\
irMn & Mean Information Retention \\
irSD & Standard Deviation of Information Retention \\
KE & Kernel Estimate \\
\hline LAI & Leaf Area Index \\
\hline Landscape \\
Less Than
\end{tabular}




\begin{tabular}{|c|c|}
\hline $\max$ & Maximum \\
\hline MAJ-1 & Majority-rule algorithm \\
\hline MDGP & Multi-Dimensional Grid Point \\
\hline $\min$. & Minimum \\
\hline Mrsh. & Marsh \\
\hline NESRS & Northeast Shark River Slough \\
\hline $\mathrm{OA}$ & Overall Accuracy \\
\hline OSI & Optimal Scaling Index \\
\hline OSIa & Optimal Scaling Index for class detection accuracy \\
\hline Prc. & Percent \\
\hline Prb. & Probability \\
\hline Prp. & Proportion \\
\hline Rch. & Richness \\
\hline Refl. & Reflective \\
\hline RL & Realized Landscape \\
\hline RND & Random rule \\
\hline RO & Random Origin \\
\hline Rpr. & Representativeness \\
\hline s & Shrub \\
\hline Smp. & Sample \\
\hline Samp. Int. & Sampling Intensity \\
\hline sB & Shrub Bayhead \\
\hline SD & Standard Deviation \\
\hline
\end{tabular}




$\begin{array}{ll}\text { SE } & \text { Standard Error } \\ \text { SF } & \text { Scale Factor } \\ \text { Sign. } & \text { Significance } \\ \text { Sprs. } & \text { Sparse } \\ \text { sptAgg } & \text { Spatial Aggregation } \\ \text { sSa } & \text { Shrub Salix } \\ \text { tB } & \text { Tree Bayhead } \\ \text { TM } & \text { Thematic Mapper } \\ \text { Txt. } & \text { Texture } \\ \text { Var. } & \text { Variables } \\ \text { WCA3A } & \text { Water Conservation Area 3A }\end{array}$




\section{INTRODUCTION}

Spatially explicit models of ecological processes across a landscape are useful for understanding naturally occurring environmental trends and disturbances or responses to management practices. Such models depend on accurate detection of spatially explicit change in land-cover at adequate spatial and temporal resolutions. Detection and monitoring of land-cover are common applications of remote sensing, but the reliable interpretation of changes in spectral reflectance patterns, either as they relate to biophysical parameters of the land surface or as changes in categorical land-cover classes, depends on the accurate identification of land-cover at the spatial, temporal and thematic precision at which changes are modeled. Often, however, spatially explicit models of change patterns integrate datasets that have been acquired and/or interpreted at different spatial scales and therefore require reconciliation of scales by either upscaling the higher- or downscaling the lower-resolution data.

The relationships between spectral reflectance patterns of electromagnetic radiation within the instantaneous field of view (i.e., pixel) recorded at a remote sensor and the biophysical parameters they relate to depend on the heterogeneity of the surface area covered by a single pixel. For landscapes that display high heterogeneity in cover types relative to the spatial resolution of the remote sensor from which biophysical parameters are derived, the integration of highly variable reflectance patterns within a pixel leads to large errors and uncertainty in the estimation of those biophysical parameters.

Complexities of spatial heterogeneity and reliable estimation of biophysical parameters using remotely sensed data have been identified and described for a suite of parameters and applications. For instance, Leaf Area Index (LAI), which estimates green 
leaf area per unit ground, and Fraction of Photosynthetically Active Radiation (FPAR), which estimates the fraction of radiant energy absorbed by of plants, are two important biophysical variables in ecosystem productivity models. Estimates of both from remotely sensed data rely on prior knowledge of land-cover, biome or vegetation type (Ganguly et al. 2012; Le Maire et al. 2012; Steltzer and Welker 2006). Consequently, as spatial resolution decreases, integrating over increasingly heterogeneous land surfaces with less precise knowledge of mixed-pixel compositions, leads to increase in error and uncertainty of estimated biophysical parameters.

Another application where scaling of land-cover information is required is the modelling of land-cover change across long temporal extents. For a specific geographic region, the classification schemes that capture frequent co-occurrence patterns of vegetation classes vary with scale. Spatial and spectral resolution of a remote sensor determine adequacy of a sensor to differentiate the classes of a classification scheme. Availability of adequate remotely sensed data at each time step, therefore, dictate the spatial and thematic resolutions and, therefore, the scales at which maps can be reliably delineated from those data. Since the spatial resolution of remote sensors increased by several magnitudes over the past four decades, low-resolution, mixed-pixel classes can now be represented by pure pixels of their constituent class components, resulting in land-cover maps with high spatial and thematic precisions. Multi-spectral datasets of sensors, available since the late 1970s, have steadily increased in spatial resolution. The datasets range from low-resolution data of about 1,000 m (e.g., Advanced Very High Resolution Radiometer), to medium resolutions of 10-50 m (e.g., Landsat, Satellite Pour l'Observation de la Terre, Sentinel 2, etc.), to high resolutions of less than $5 \mathrm{~m}$ for commercial and private 
satellites (e.g., RapidEye, WorldView, GeoEye, etc.). Airborne multi- and hyper-spectral sensors mounted on manned or unmanned aerial platforms even allow for mapping at very high sub-meter resolutions. To combine categorical land-cover maps that were derived at different spatial resolutions, a reliable scaling algorithm is needed that retains as much information as possible from the higher resolution, while generating representative classification schemes that are valid at the lower spatial resolutions.

However, the most common aggregation methods to match categorical vegetation maps to the scale at which biophysical parameters are often analyzed, or at which landcover changes are monitored, are the mode or majority rule, the nearest-neighbor rule, and the random rule. Since none of these spatial aggregation methods account for scale sensitivity of classification systems, their application leads to uncontrolled loss of information content in aggregated maps, and, subsequently, ecological fallacy in ecological models that make use of these aggregated data. Spatial scaling of categorical data, therefore, needs to be combined with re-classification and substitution of the original classification system. No spatial aggregation algorithm, to date, considers re-classification of the categorical class system.

This dissertation develops and explores application of a new spatial scaling algorithm that accounts for scale sensitivity of classification systems and information loss as spatial data aggregation occurs. Chapter I addresses the requirements of a scaling algorithm that produces representative classification schemes at specific scales. Developing representative classification systems from quantitative measures of species cooccurrence patterns has a long history in the fields of phytosociology, vegetation classification and community ecology (Braun-Blanquet 1964; Van Der Maarel 1979). 
Drawing from these sub-disciplines of ecology, a new scaling method and an algorithm to implement the method were developed. In a first step, the theoretical sample space of gridbased categorical data was explored and characteristics of landscape and scaling parameters that affect the sample space were identified. On the basis of the sample space, the new algorithm was developed. A testing framework was introduced that allowed for testing of the effects that landscape characteristics and scaling parameters have on algorithm performance. Effects were tested for a set of simulated neutral landscapes with known properties and a range for each of the scaling parameters.

In Chapter II, the proposed scaling algorithm was applied to high-resolution categorical raster datasets to evaluate the effects of algorithm parameters on scaling consistency and detection of scaled classes from multi-spectral satellite datasets with lower resolution than the scaled high-resolution maps. The goal was to optimize scaling parameters to reduce information loss, increase classification scheme consistency, and maximize classification accuracy. Effects were evaluated for two natural landscapes within the greater Everglades (FL, USA) ecosystem whose vegetation had been mapped from WorldView-2 (WV-2) multi-spectral data at a spatial resolution of $2 \mathrm{~m}$. The maps were scaled to $30 \mathrm{~m}$, the resolution of multi-spectral Landsat data and detectability of the scaled classes from Landsat data was evaluated.

Because successful scaling of high-resolution categorical maps relies on accurate representations of the landscape, Chapter III assessed effects of classification methods on classification accuracy, confidence and method efficiency. In chapter III, I explored the trade-offs of training sample size and feature-space dimensionality on overall accuracy, location-specific classification confidence, class-specific accuracies and classifier training 
and prediction times. A framework to model the interactions of training sample intensity, feature space, and their effects on mapping accuracies and processing efficiency was developed and then used to optimize a mapping method for wetland plant communities from high-resolution multi-spectral satellite data. 


\section{REFERENCES}

Braun-Blanquet, J. 1964. Pflanzensoziologie, Grundzüge Der Vegetationskunde. 3. Auflage. Wien: Springer Verlag.

Ganguly, S., R. R. Nemani, G. Zhang, H. Hashimoto, C. Milesi, A. Michaelis, W. Wang, et al. 2012. "Generating Global Leaf Area Index from Landsat: Algorithm Formulation and Demonstration." Remote Sensing of Environment 122:185-202. https://doi.org/10.1016/j.rse.2011.10.032.

Le Maire, G., C. Marsden, Y. Nouvellon, J. L. Stape, and F. J. Ponzoni. 2012. "Calibration of a Species-Specific Spectral Vegetation Index for Leaf Area Index (LAI) Monitoring: Example with MODIS Reflectance Time-Series on Eucalyptus Plantations.” Remote Sensing 4 (12):3766-80. https://doi.org/10.3390/rs4123766.

Steltzer, H., and J. M. Welker. 2006. "Modeling the Effect of Photosynthetic Vegetation Properties on the NDVI-LAI Relationship.” Ecology 87 (11):2765-72. https://doi.org/10.1890/0012-9658(2006)87[2765:MTEOPV]2.0.CO;2.

Van Der Maarel, E. 1979. "Transformation of Cover-Abundance Values in Phytosociology and Its Effects on Community Similarity." Plant Ecology 39 (2):97-114. https://doi.org/10.1007/bf00052021.

Zhao, J., Y. Wang, H. Zhang, Z. Zhang, X. Guo, S. Yu, and W. Du. 2016. "Spatially and Temporally Continuous LAI Datasets Based on the Mixed Pixel Decomposition Method.” SpringerPlus 5:516. https://doi.org/10.1186/s40064-016-2166-9. 


\title{
CHAPTER I
}

\section{QUANTITATIVE SPATIAL UPSCALING OF CATEGORICAL INFORMATION: THE MULTI-DIMENSIONAL GRID POINT SCALING}

\begin{abstract}
ALGORITHM
Definitions (in alphabetic order)

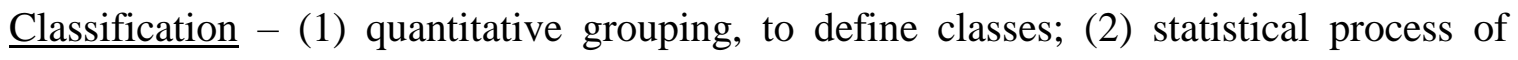
assigning objects or sample units to pre-defined categorical classes.

Community - Frequently occurring association patterns of plant species with similar relative abundances and physiognomic characteristics for spatially defined geographic units (grain size) within a defined regional extent (landscape).

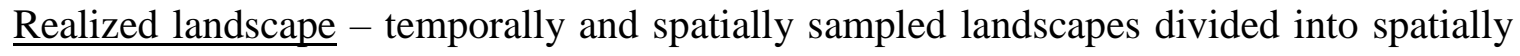
and temporally discrete units through quantization of space and time continuum.

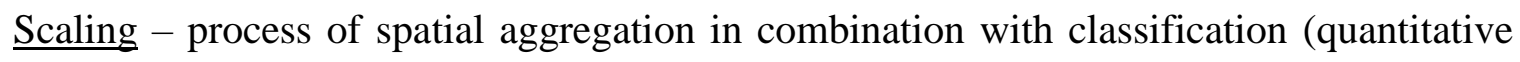
grouping).

Spatial aggregation - (1) spatial distribution of objects across a landscape; antonym: dispersion; (2) process of aggregating smaller geographic units into larger units, assigning new values based on a decision-rule algorithm.
\end{abstract}

\section{INTRODUCTION}

Spatially explicit ecological models rely on spatially exhaustive data layers that have appropriate scales for the ecological processes of interest (Lam and Quattrochi 1992; Mas, Gao, and Pacheco 2010; Quattrochi 1991; Sayre 2005). Such exhaustive data layers are often categorical raster maps that were derived from high-resolution, remotely sensed 
data and that require upscaling to a lower spatial resolution to make them compatible with the scale of ecological analysis. Quantitative scaling of spatially explicit, continuous, raster datasets has received considerable attention (Wu and $\mathrm{Li}$ 2009), but several issues associated with the scaling of categorical data have not been resolved. Statistical functions for spatial data aggregation that are meaningful for continuous data include sum, minimum, maximum, mean or median values of local neighborhoods, but none of these functions are valid for categorical data.

Statistical functions most often used when aggregating categorical data are the mode, also known as the majority rule, the nearest-neighbor rule and the random rule. Majority rule follows the simple majority or plurality decision rule, where the assigned output class label is the input class with the highest proportion of sub-samples (mode); the nearest-neighbor rule assigns the class label of the original cell closest to the center of the scaled grid cell; and the random rule assigns the output class at random from the classes present within the output grid cell, either with the same probability for each class present or with probability proportional to class abundance. Application of these three algorithms to the same input data can result in different class assignments of the up-scaled grid cell (Fig. 1.1).

Two critical issues arise with the use of these functions: (1) ignoring large portions of information present in the high-resolution grid cells leads to high uncontrolled loss of information in the scaled dataset; and (2) maintaining classes from the high-resolution dataset at the lower spatial resolution assumes validity of the classification scheme at the lower resolution, failing to represent recurring mixes of heterogeneous classes present in 
lower-resolution grid cells (Fig. 1.1). The new scaling method proposed in this paper addresses both of these issues.

\section{Spatial Data Aggregation Algorithms and Information Loss}

Spatial upscaling of categorical raster data aggregates information to a coarser spatial resolution by combining information from multiple original map objects (i.e., pixels, grid cells) from the initial resolution. As data are aggregated, information is generalized, leading to information loss (He, Ventura, and Mladenoff 2002; Turner 1989). Different spatial aggregation methods result in very different aggregated map products (Gann, Richards, and Biswas 2012; Ju, Gopal, and Kolaczyk 2005), over- or underestimating class abundances and often oversimplifying complex spatial patterns (Francis and Klopatek 2000; O’Neill et al. 1988; Wu 1999, 2004). As a result of generalization, disjunction of information between scales can result in misleading conclusions about extant landscape patterns and their changes over time (Johnson et al. 2001; Lam and Quattrochi 1992; Mas, Gao, and Pacheco 2010; O’Neill et al. 1996; Ostapowicz et al. 2008; Riitters et al. 1995; Scheiner et al. 2000; Tischendorf 2001; Turner 1989; Wu et al. 2002).

Incoherence between fine-scale maps and their spatially aggregated versions needs to be addressed to avoid faulty inference from models that use spatially aggregated categorical maps that are not representative at the scales that they are generated. Since aggregation generalizes with the goal to maintain only information that is crucial to the analysis of a phenomenon at the aggregated scale, it is of interest not only to quantify the amount of information retained in the aggregated product, but also to control information loss. Hence, to achieve optimal levels of generalization relevant to a scientific question, it 
is essential for an aggregation algorithm to control information loss in a predictable and reproducible fashion and to quantify the information retained in the scaled product in a spatially explicit fashion.

Classification schemes are only valid for the range of spatial scales for which they were defined. Commonly applied spatial aggregation methods, such as the majority rule, nearest-neighbor rule, and random rule, do not account for scale sensitivity of classification systems. These algorithms tend to increase abundance of dominant classes and reduce or eliminate rare classes (He, Ventura, and Mladenoff 2002) (Fig. 1.1), and even more complex spatial aggregation methods, such as scan statistics (Coulston et al. 2014) only consider the original, high-resolution, classification system classes when class labels are assigned to the scaled, larger, spatial units. These aggregation methods presume validity of class descriptors for the aggregated lower-resolution product, regardless of the scaling factor, which leads to uncontrolled loss of information content in aggregated maps (Fig. 1.1), and, subsequently, ecological fallacy in ecological models that make use of these aggregated data.

Scaling methods that acknowledge class variability with spatial scales often use hierarchical class systems that aim at generating more general classes as aggregation occurs (Wu and David 2002). Ju et al. (2005) developed the multi-scale, multi-granular framework that allows for scaling in the spatial domain using quad-tree data structures to increase flexibility for aggregation in the spatial domain and in the categorical domain. The categorical domain, however, was limited to hierarchical class labels that aggregate to coarser, all-inclusive, pre-determined class labels (Ju, Gopal, and Kolaczyk 2005). Hierarchical classification systems, by definition, aggregate linearly. However, co- 
occurrence patterns of classes can result from processes that operate at different scales and do not necessarily lead to hierarchical systems. Hierarchical classification systems, therefore, over-simplify complex patterns of spatial heterogeneity and mechanisms that lead to co-occurrence patterns. In addition, hierarchical systems often lack the quantitative definitions required for automated scaling or aggregation algorithms. Spatial scaling of categorical data, therefore, needs to go beyond aggregation of spatial units. It needs to be conducted in combination with re-classification (grouping) of the original classes of the high-resolution classification system. No aggregation algorithm, to date, considers these aspects of spatial aggregation of categorical data.

An aggregation algorithm that generates scale-specific classification schemes as spatial aggregation occurs needs to be consistent in delivering class descriptors (labels) that are reproducible and representative for the entire population. In the case of rasterized landscapes, representativeness is the recognition of entities or classes that recur frequently at a specific scale. To attain adequate precision in the thematic domain that supports the use of the aggregated product in subsequent ecological models, the scaling algorithm should also provide a control mechanism for information retention in the thematic domain as the scaled classification scheme is generated.

\section{Ecology - the Unifying Framework for Scaling}

Several sub-disciplines of ecology have addressed similar issues that arise with scaling of categorical data. Developing representative classification systems that are based on quantitative measures (i.e., species co-occurrence patterns) has a long history in the field of phytosociology, vegetation classification and community ecology (Braun-Blanquet 1932, 1964; Van Der Maarel 1975, 1979). The processes that determine co-occurrence of 
plant species within a geographic region and the meaning of the term "communities" are debated. What determines the meaning of the term community is whether community is perceived as a climax community (Clements 1916), as individuals of species associating independently in response to environmental gradients (Gleason 1939; Tansley 1935; Whittaker 1962), or as co-location of individuals that are mainly driven by stochastic events (Alonso and McKane 2004; Hubbell 2005, 2006; Rosindell, Hubbell, and Etienne 2011). However, regardless of which processes are responsible for community assembly, the use of the term "community" as a reference to recurring patterns of plant associations is practical and meaningful for operational purposes (Boutin and Keddy 1993; Keddy 1992, 1993). The definition of the term "community" itself depends on the interest and focus of a study (Parker 2001) and, therefore, relies on a set of clearly defined criteria.

In the context of categorical data scaling, more important than the processes that lead to association patterns is the recognition that the quantitative co-occurrence patterns of species or vegetation types are dependent on spatial scale. Association patterns of species, when randomly sampled on a $1-\mathrm{m}^{2}$ scale across a defined spatial extent are expected to differ from the association patterns of the same species on a $50-\mathrm{m}^{2}$ scale (O'Neill et al. 1996; Schlup and Wagner 2008). Consequently, plant community classifications vary along the continuum of spatial scales. Recognizing this variability in association patterns at different spatial scales, when upscaling and aggregating classes, captures the results of the various processes that lead to association patterns at the different scales (Cingolani et al. 2007; Shipley and Keddy 1987).

Multi-scale, non-hierarchical classification schemes that allow vegetation classes to account for the full variability of co-occurrence patterns across scales are more apt to 
account for patterns that vary across scales, especially when vegetation responses to environmental gradients and importance of stochastic events across scales are unknown. Such classification systems allow for a more dynamic view of changing landscapes (e.g., novel landscapes) at multiple scales. Common aggregation methods do not recognize this need for non-hierarchical, scale-specific, classification systems and ignore ecological concepts of community assembly and scale dependence of patterns and processes (Jonsson, Moen, and Gunnar 1998), and, therefore, fail to recognize the possible invalidity of their aggregates.

Samples drawn from a categorical raster map for a specific grid cell size (e.g., $3 \times 3$ aggregation kernel) resemble relevé data of species abundance for quadrats or plots, where each sampled grid cell represents a plot of a relevé set. The process of classification or quantitative grouping of relative species abundance of relevé plot data results in vegetation or community classification schemes. Hence, a spatial aggregation algorithm that is tasked with the generation of a classification scheme faces similar challenges identified in the field of phytosociology: (1) how to classify communities or vegetation consistently when using sample data; (2) how to validate classes and class systems across the larger landscape; and (3) how to reliably assign unknown samples to pre-defined classes (De Cáceres and Wiser 2012; De Cáceres et al. 2009; Tichý, Chytrý, and S̆marda 2011; Tichý et al. 2010; Wildi 2010).

A commonly used statistical grouping method is cluster analysis. Most of the classic cluster methods that have been used in community ecology and phytosociology consist of a two-step procedure. The first step establishes a measure of similarity or dissimilarity between all samples, which is usually based on distance metrics (e.g., 
Euclidean, Manhattan, Canberra, etc.). In a second step, samples are organized into clusters (groups) with either some form of hierarchical, k-means, density or probabilitybased algorithm. Distance-based algorithms aim at grouping objects to maximize distance of group object attributes and to minimize distances among objects assigned to the same group. The number of statistically significant supported clusters can be determined based on a variety of validity indices (Arbelaitz et al. 2013).

Accurate detection of vegetation requires that classification results are reproducible and consistent in determining a reasonable number of clusters that are representative of common vegetation-association patterns (De Cáceres and Wiser 2012; De Cáceres et al. 2009; Mucina, Schaminée, and Rodwell 2000). However, cluster results are highly dependent on cluster algorithm, distance metric and index selection, which leads to low confidence in cluster results. Sampling error of cluster results has been addressed using bootstrap methods (Tichý, Chytrý, and S̆marda 2011), but representativeness of resulting classification systems derived from only a small sample across the larger landscape are rarely evaluated. Further, the number of statistically supported clusters or classes is highly dependent on the nature of the underlying data pattern and its effects on the statistical methods that are applied (i.e., methods that rely on distance metrics). It is therefore crucial to understand these effects to evaluate the applicability and limitations of these methods (Robertson 1980).

\section{Defining the Sample Space}

Data type, measurement level, and distribution patterns of the data have a strong effect on statistical results, which means that no method is universally superior or inferior (Milligan and Cooper 1985; Vendramin, Campello, and Hruschka 2010) and that data 
analysis methods, including scaling algorithms, need to be evaluated in context. Statistical data type, level of measurement, and distributions that rise from sampling a specific sample space are critical when selecting appropriate mathematical and statistical methods for inference.

The sample space in the case of spatially explicit categorical data, as presented in raster maps, is a finite, discrete, sample space, where samples for spatial sub-units of local neighborhoods result in count frequencies of classes. This sample space depends on (1) the spatial and thematic characteristics of the landscape and (2) the scale factor. To develop a valid algorithm and to be able to evaluate algorithm performance, it is essential to understand how landscape characteristics and scale factor affect the sample data type and distributions.

Two landscape characteristics that have an effect on the relative abundance distributions of samples are diversity and spatial arrangement or configuration of a landscape. Diversity is represented by richness $(r c h)$ and evenness, while the spatial distribution patterns of the classes across the landscape can be summarized with a spatial aggregation metric (sptAgg) (referring to the characteristic, not the process). Richness simply relates to the number of distinct object types that are differentiated at the sample scale. For vegetated landscapes, object types can refer to species, assemblages, communities, or vegetation classes. Evenness refers to the relative abundance of object types (i.e., species or classes) across the landscape. Numerous ecological models attempt to explain the shape of relative species-abundance distributions, and mathematical models (e.g., log-normal series, log-series, or geometric series) are fit to model these distributions (McGill et al. 2007). Spatial aggregation, as a characteristic of the landscape, refers to the 
spatial distribution of object types and can range from systematic to random and from highly dispersed to completely aggregated, when the number of patches is equal to the number of classes.

Scale factor $(s f)$ is the ratio of the spatial resolution of the scaled grid to the resolution of the original or high-resolution grid (Eq. 1.1), and the number of sub-units or grid cells within a sample $\left(N_{s m p}\right)$ is the scale factor squared (Eq. 1.2).

$$
\begin{aligned}
& s f=\frac{\text { resolution }(\text { scaled grid })}{\text { resolution(original grid) }} \\
& N_{s m p}=s f^{2}
\end{aligned}
$$

For instance, if the scaled grid resolution is $10 \mathrm{~m}$ and the resolution of the original data grid is $2 \mathrm{~m}$ then $s f=5$ and the number of original cells in each scaled sample unit is 25 . Let richness $(r c h)$ be greater than 1 and the scale factor $(s f)$ be a positive integer with odd parity greater than 1 (i.e., $\{3,5,7 \ldots\}$ ). Then, the number of possible distinct outcomes of a sample is the number of restricted or weak compositions with binomial coefficients,

$$
\left(\begin{array}{c}
N_{s m p}+r c h-1 \\
r c h-1
\end{array}\right), \text { or } \frac{\left(N_{s m p}+r c h-1\right) !}{(r c h-1) ! *\left(N_{s m p}+r c h-1-(r c h-1)\right) !} \quad \text { Equation } 1.3
$$

Since percent-cover per sample is constrained to exactly $100 \%$ (exclusive and exhaustive cell occupancy, without vertical stratification), the precision of relative class abundances is calculated according to equation 1.4.

$$
\operatorname{Prc}_{c}=\frac{100}{N_{s m p}}
$$

Equation 1.4

For instance, let $r c h=2$, and apply a scale factor $s f=3$ (kernel of $3 \times 3$ ). The number of sample units $N_{s m p}=9$ and the unique sample compositions (combinations of relative cover options) is 


$$
\left(\begin{array}{c}
9+2-1 \\
2-1
\end{array}\right), \text { or } \frac{(9+2-1) !}{(2-1) ! *(9+2-1-(2-1)) !}=10
$$

The 9 sub-sample units with 2 class occupancy options for each sub-sample has 10 possible frequency combinations of the two classes (i.e., 4,5; 5,4;3,6;6,3; 7,2; 2,7; 8,1; 1,8; 9,0 and 0,9) with a class precision of $\operatorname{Prc}_{c}=\frac{100}{9}=11.1 \%$. Thus, there are 10 possible new classes or class labels for the scaled grid cell.

Table 1.1 presents the relationship of the richness and scale factor to the number of constrained unique compositions and their sample precisions. For a given $s f>1$, as a landscape increases in richness (Tbl. 1.1, columns), or for a given $r c h>1$, as $s f$ increases (Tbl. 1.1, rows), the number of unique compositions per sampling unit increases rapidly. Precision of relative cover differences (percent intervals) is determined by scale factor only (Eqs. 1.1, 1.2 and 1.4) and increases with $s f$. For a given landscape, the frequency distribution of each possible composition with specific richness and scaled with a specific scale factor is then a function of diversity, which includes evenness of the class distribution and spatial class aggregation across the landscape.

Presence and frequency of zeroes in the sample data also depend on landscape and sampling characteristics. For example, for $s f=3$, the number of sub-sample units $=9$, and if $r c h=10$, every sample will produce compositions with at least one class being absent. Consequently, each observation includes at least one zero in the sample vector. Observations with zeroes are therefore anticipated whenever the scale factor is small relative to the number of classes and are always present when a scale factor produces a sub-sample unit count less than the number of original classes. The number of zeroes in 
each observation is expected to increase with richness and spatial aggregation of classes across the landscape.

In summary, when sampling categorical raster models of landscapes with a square grid of dimensions $n \times n$ with $n>1$, richness and scale factor determine the number of possible sample compositions, and evenness and spatial aggregation characteristics of the landscape determine the expected frequency distributions of each possible sample combination. The constraint that the sum of all sample proportions $=1$ makes the data compositional in nature. Further, the presence of zeroes in any given observation is very probable. Hence, a scaling algorithm that generates classification schemes as spatial units are aggregated has to be robust in dealing with compositional data (i.e., data that are percentages or proportions) and with the presence of a high frequency of zeroes in the data samples.

\section{Simplex Space}

The sample space of compositional data is called the simplex or $\mathrm{S}^{\mathrm{D}}$ (Aitchison 1986).

$$
S^{D}=\left\{\mathrm{x}=\left[x_{1} ; \ldots ; x_{D}\right] \mid x_{i} \geq 0 \text { and } \sum_{i=0}^{D} x_{i}=\kappa\right\}
$$

Simplex space is constrained to all $x_{i} \geq 0$, and the sum of all $x_{i}=\kappa=1$. Compositional data are scale invariant, consequently, statistical methods that group objects should result in the same groups and class labels if relative compositions are the same and only totals differ (e.g., total area sampled as scale factor increases). When dealing with compositional data, and more specifically, count compositions (i.e., integers), quantitative grouping or classification methods (e.g., cluster algorithms, discriminant functions, etc.) that use 
distance metrics of the real space $\mathrm{R}$ and that assume multivariate normal distributions are inadequate (Van Den Boogaart and Tolosana-Delgado 2008). Proposed solutions for statistical analysis of compositional data are log-ratio transformations of compositional data (Aitchison 1986; Aitchison and J. Egozcue 2005). Several log-ratio transformations have been proposed to convert compositional data from the simplex space $\mathrm{S}$ of $\mathrm{D}$ dimensions to the real space R with D+1 dimensions (Aitchison 1986; Egozcue et al. 2003), which then allows for application of analytical methods that are valid in $\mathrm{R}$ space. The alternative approach is to stay in the simplex space for data analysis, which has the advantage that the modeled relationships of data structures are direct and more comprehensible. The distance that is valid in the simplex space is the Aitchison distance, $d_{A}$.

$$
d_{A}=\left[\sum_{k=1}^{D}\left(\log \left(\frac{x_{h k}}{g\left(x_{h}\right)}\right)-\log \left(\frac{x_{i k}}{g\left(x_{i}\right)}\right)\right)^{2}\right]^{\frac{1}{2}}
$$

The geometric mean of the composition $\mathrm{x}$ (Aitchison 1986) is then

$$
g(x)=\left(\prod_{k=1}^{D} x_{k}\right)^{\frac{1}{D}}
$$

Log-ratio transformations and Aitchison distance, however, are not valid for compositions with components of zero and several methods have been proposed to deal with zero count data (Aitchison et al. 2000; Martín-Fernández, Barceló-Vidal, and Pawlowsky-Glahn 2003; Martín-Fernández et al. 2014). In the case of true absence, though, any of the proposed methods adds noise to the data and suggests presence of a class when, in fact, the class is absent. 
In summary, common spatial aggregation algorithms (e.g., majority rule, random rule) lack robust quantitative scaling mechanisms that acknowledge scale-dependence of classification systems. In addition, currently used algorithms do not estimate spatially explicit information loss or allow for control of information retention (thematic precision), potentially leading to excessive loss of information when scaling across large spatial units. Common statistical procedures and methods to establish vegetation classifications from sample data (i.e., cluster algorithms that rely on Euclidean distance metrics) are unsuitable for the data patterns that arise from the grid sampling design of categorical data, that produce multivariate compositional data structures with zero variance (sum 1 constraint), and that have a high frequency of zeroes.

Therefore, the new scaling algorithm proposed here aims at conducting spatial aggregation of categorical data (e.g., land cover, land use, vegetation classes), while (1) simultaneously generating a non-hierarchical representative classification system valid at the aggregated scale and (2) allowing for control of information loss.

\section{The Multi-Dimensional Grid Point (MDGP) Scaling Algorithm}

The proposed scaling algorithm performs two main tasks: (1) the classification (grouping) of landscape objects that is valid for the scale of spatial aggregation, resulting in a scale-specific classification system; and (2) classification (assignment) of spatially aggregated units of the landscape to one of the classes of the new classification system. The first task creates a valid classification system at the specified scale, while the second task assigns the new classes in that system to the aggregated units of the finer scale data. The two tasks are integrated. 
The algorithm recognizes the multi-dimensional feature space spanned by the compositional data structure space $S^{D}$, the polyhedron, where the number of variables or features of the polyhedron define the vertices (i.e., richness, the number of original classes). Number and location of the regularly spaced grid points in the solid space of the polyhedron represent the finite number of discrete unique count compositions for a given richness and scale factor (Tbl. 1.1, Fig. 1.2). As richness and scale factor increase, the number of possible class combinations (i.e., scaled richness) increases (Tbl. 1.1, Fig. 1.2), leading to an apparent shift from count composition (multinomial) distributions to distributions that resemble the multi-dimensional continuous scale (Van Den Boogaart and TolosanaDelgado 2008). As the number of possible output classes rapidly increases to an unmanageable number, however, precision of the potential output class labels increases beyond ecological and, in many cases, statistical significance (Tbl. 1.1, Precision).

The solution to reducing insignificant precision that is offered by the proposed algorithm is to limit the label precision by implementing a partitioning parameter, which reduces the number of grid points in the compositional feature space. The precision parameter partitions the sample space (0-100\%) into x equal parts for each class, for which class precision $\operatorname{Prc}_{c}=100 / x$. The result is a polyhedron with regularly spaced multidimensional grid points, where the number of dimensions is still equal to the number of features (i.e., classes), but the number of grid points is now limited by the number of equal parts instead of by the scale factor, thus reducing the number of possible weak combinations. The effects of the partitioning parameter on the number of grid points and class-label precision are demonstrated in Table 1.2 and Figure 1.2. Comparing Tables 1.1 
and 1.2 illustrates the reduction in number of theoretical grid points and its effect on thematic class precision.

The second step, classification or assignment of each scaled grid cell to a multidimensional grid point, requires a decision rule. The decision criterion proposed here is the minimization of information loss. Let information loss be defined as the difference between relative frequency distribution of the sample data and the generalized, scaled representation, i.e., the multi-dimensional grid point. The percent agreement of each class component in the abundance data and the grid point percentage constitutes the percent agreement of information; the reduction of information in an aggregated unit is then the difference between the abundance data (converted to proportions or percent) of the sample and the nominal aggregation percentages of the generalized grid point. When none of the original classes are represented in the generalized class, agreement is zero with no information retained; if all proportions of the original composition are maintained in the aggregated class label, agreement is $100 \%$, and no information loss has occurred.

Hence, information retention (IR) for each scaled grid cell can then be defined as the sum of minimum agreement of each class component in the sample $(S m p)$ and the grid point $(G P)$, where $P_{i}=$ relative abundance of class $i$ in percent and $N=$ the number of classes in the sample data of the scaled grid cell.

$$
I R=\sum_{i=1}^{N} \min \left(\mathrm{P}_{i} S m p, \mathrm{P}_{i} G P\right)
$$

For instance, implementing a class-label precision of 50\% (part $=2)$ for a classification scheme of three original classes A, B, and C, produces six 3-dimensional grid points, including the following two combinations: A50_B50_C0 $(\mathrm{A}=50 \%$, class $\mathrm{B}=50 \%$ and 
class $\mathrm{C}=0 \%)$ and A50_B0_C50 $(\mathrm{A}=50 \%$, class $\mathrm{B}=0 \%$ and class $\mathrm{C}=50 \%)$. Calculating IR for a grid sample with abundance percentages of class $A=40 \%$, class $B=10 \%$ and class $\mathrm{C}=50 \%$ results in $50 \%$ and $90 \%$ IR for grid points one and two, respectively. Assigning the sample to grid point two maximizes IR, which is equivalent to minimizing information loss. The integrated class-specific and landscape-level retained information content can then be estimated with standard descriptive statistical parameters (mean, standard deviation, median and percentile ranges). Information retention is expected to be positively correlated with the partition or precision parameter and thus to function as a control parameter for information loss. Since IR is a bounded metric $(0-100 \%)$, independent of class number and scale factor, it can be compared across scaling results of different algorithms.

A third aspect, class representativeness, was introduced to further reduce the number of final classes in the scaled classification scheme. To allow for removal of nonessential scaled classes that have a very low frequency across the re-scaled landscape, the minimum representativeness threshold parameter was implemented. Applying this parameter, output classes (grid points) that are below the user-defined threshold are iteratively removed, and the grid cells that had been assigned to them are re-assigned to the remaining grid point that maximizes IR. The process repeats until no class is below the representativeness threshold. The threshold of a minimum percent representativeness across the landscape is optional but meaningful, especially when the non-representative classes are not of interest ecologically.

A fourth parameter, also optional, allows for maintaining homogenous classes if they fall below the representativeness threshold and would be eliminated in the process of 
grid point removal. The homogeneity threshold defines is the minimum class percentage in a sample to declare it a homogenous or monotypic class. If samples are encountered where the homogeneity threshold is reached for any of the classes, the grid point representing that class will be maintained in the final class set as a homogenous class $(100 \%)$, even if it is below the landscape level representativeness threshold, which allows for retention of rare classes.

\section{Application of the MDGP-Scaling Algorithm to Categorical Raster Data}

Applying the MDGP-scaling algorithm to a categorical raster map requires six inputs. These are a categorical raster map, scale factor (sf), the grid origin of the aggregated map, the partitioning parameter (part) representing the thematic class-label precision, and the optional threshold parameters for landscape representativeness threshold and homogeneity threshold as proportion. The automated steps of applying the algorithm to a raster map are:

(1) Generate a kernel with dimensions $s f$ x $s f$.

(2) Generate all enumerated scaled grid cells for the given scale factor $(s f)$ for either a random origin (random selection of cells in the top left quadrant of the map to account for edge effect) or a user-defined origin. The number of grid cells accounting for edge effects is the number of original cells minus the edge of the kernel along all four edges divided by the scale factor.

$$
\frac{\operatorname{cell} s(x)-\frac{2 *(s f-1)}{2}}{s f} * \frac{\operatorname{cells}(y)-\frac{2 *(s f-1)}{2}}{s f}
$$

(3) Extract cell values from map for all enumerated scaled grid cells.

(4) Generate relative abundance of extracted values for all scaled grid cells. 
(5) Generate multi-dimensional grid points $(M D G P)$ based on user-defined partitioning parameter (part) that determines the thematic class precision of significant difference in relative percent cover (Tbl. 1.2, Fig. 1.3).

(6) Remove scaled grid cells that are above the homogeneity threshold, calculate IR (Eq. 1.8) and add to a list of homogenous classes.

(7) Generate IR for each heterogeneous composition in relation to all nominal MDGP compositions (Eq. 1.8).

(8) Assign each scaled grid cell to the MDGP that maximizes IR.

(9) Calculate relative abundance distribution of all MDGP classes (scaled output classes).

(10) Remove the class (grid point) with the lowest percentage cover that is below the user-defined threshold unless the class is on list of homogenous classes.

(11) Repeat steps (7) to (10) until all classes are above the landscape representativeness threshold and all scaled grid cells are assigned to a grid point.

(12) Generate class descriptors (labels) for each grid point based on class dominance and percent rounded to the nearest integer (e.g., A60_B20_C20 represents a grid point with 3 dimensions (original classes A, B, and C) and precision of $20 \%($ part $=5$ ) with class abundance $A=60 \%$, class $B=20 \%$ and class $\mathrm{C}=20 \%$ ).

(13) Assign class labels to each $M D G P$-classified scaled grid cell.

The objective of the following simulation study was to compare spatial aggregation products of commonly applied aggregation algorithms to the proposed MDGP-scaling 
algorithm and then to conduct a consistency and sensitivity analysis of the MDGP-scaling to the scaling parameters scale factor and class-label precision.

\section{METHODS}

Validity and efficacy of the MDGP-scaling algorithm was evaluated in comparison to other commonly applied aggregation algorithms. It was also assessed with respect to consistency and sensitivity of information retention, class count and class-label fidelity as a function of scaling parameters and landscape properties. To evaluate the proposed algorithm and compare it to other algorithms, a conceptual framework was developed that included a simulation component to generate artificial neutral random landscapes with known properties. The landscape properties or characteristics that were controlled were richness, class-abundance distribution (CAD) and spatial aggregation of classes across the landscape. Landscapes were simulated in a full factorial design for a range of values for each of these properties.

\section{Test Framework: Neutral Landscape Models}

Neutral models have been used in ecology to explore interactions between processes and patterns (Gotelli and Graves 1996; O’Neill et al. 1988). In the landscape context, neutral models have been expanded to the spatial domain, where neutral refers to random landscape models with known properties that are process neutral (Gardner 1991; O’Neill et al. 1988). Testing the performance of algorithms on samples taken from complex landscapes with known properties sets the statistical benchmark for applying them to real populations (With and King 1997). Generating multiple replicas of landscapes with similar properties but that vary in spatial pattern allows for evaluation of consistency and sensitivity of algorithms to the variability of a variety of properties (Fahrig 1991). The 
test framework integrated the generation of neutral landscape models, scaling of the highresolution landscapes, and evaluation of scaling results when applied to the different combinations of landscape properties and scaling parameter options. The framework consisted of three sub-routines (Fig. 1.4):

(1) Generation of random neutral landscapes using the Python library nlmpy, in a full factorial design of value lists that were provided for the four parameters:
a. Landscape model type $\{$ mid-point OR cluster $\}$
b. Richness (class number) $\{2,3 \ldots$ )
c. Spatial aggregation weight $\{$ mid-point: $0-1$; cluster: $0-0.58\}$
d. Class-abundance distribution model \{ equal, geometric, log-series, gamma, negative binomial, log-normal \}

(2) Raw sample data extraction and grid-cell level tabulation of relative class abundances for each generated landscape and scale factor. Required inputs are:

a. Scale factor list $\{$ odd integers $\}$

b. Random origin count $\{$ integer $\}$; Random origins were generated from a list of cells that were in the northwest quadrant of the first aggregation cell, including the center of that cell (e.g., for scale factor 3, the list of 9 cells was limited to row and column $(1,1 ; 1,2 ; 2,1 ; 2,2)$.

c. For each scale factor and random origin, grid values are extracted from each landscape and relative class abundances are tabulated for all lower resolution grid cells. 
(3) Scaling of the data to generate new class labels for the scaled output grid and an associated classification scheme for a set of algorithms of interest. Required parameters are:

a. Scaling algorithm list $\{$ majority, random, $M D G P\}$

For the $M D G P$-scaling algorithm, additional parameters are:

b. Class-label precision list $\{2,3 \ldots\}$; At least one is required

c. Landscape representativeness as proportion $\{0-1\}$; Optional with default 0.01

d. Monotypic class threshold as proportion $\{0-1\}$; Optional with default 0.9

e. Each algorithm listed is applied to the set of cell-specific relative abundance data for each landscape, and evaluation variables for analysis are generated and written to file.

The framework (Fig. 1.4) implements a full factorial design of all parameter lists. The number of landscapes that are generated is the number of class-abundance distributions $\mathrm{x}$ the richness levels $\mathrm{x}$ the number of spatial aggregation levels of classes $\mathrm{x}$ the number of replicates of each landscape. The number of scaled landscape versions is then the number of landscapes $\mathrm{x}$ the number of scale factors $\mathrm{x}$ the number of random origins for scaling $\mathrm{x}$ the number of class-label precisions.

\section{Landscape Parameter Settings for Simulation}

For the simulation study, neutral landscape models were produced with a mid-point replacement algorithm (Fournier, Fussell, and Carpenter 1982; Palmer 1992), which produces landscapes that resemble landscapes with environmental gradients. Variability 
of spatial patterns was generated for three levels of richness (3, 6, and 9 classes), two models of evenness (equal and geometric CAD models), and four levels of spatial aggregation $(0,0.3,0.6,1)$. Equal abundance of cover types, although very unlikely in natural systems, provided the most neutral random landscape type. The equal distribution models were contrasted with simple geometric class distribution models on the basis of ecological resource limitation theory (Motomura 1932), but any other statistical distribution model (e.g., log-series, gamma, negative binomial, log-normal) could have been implemented. Proportions of class presence across the landscape were calculated according to equation 1.10 for equal class distributions and according to equation 1.11 for the geometric class distributions.

$$
\begin{aligned}
\mathrm{P}_{c} & =\frac{1}{N} \\
\mathrm{P}_{c} & =\frac{2^{N-1}}{\left(2 * 2^{N-1}-1\right) * 2^{c-1}}
\end{aligned}
$$

Equation 1.10

The total number of classes is $\mathrm{N}$, and $\mathrm{P}_{c}$ is the proportion of class $c$. Spatial aggregation for the landscapes was achieved with class aggregation parameters. For the mid-point replacement models, aggregation parameter $p$ ranges from 0 to 1 . For this study, four levels of spatial aggregation were evaluated: 0 for low aggregation and $0.3,0.6$ and 1.0 for increasingly more aggregated landscapes.

A full factorial design, using the three richness levels, two class distribution models and four spatial aggregation settings, defined 24 landscape types. For a subset of 12 of the 24 landscape types, Figures 1.5 and 1.6 illustrate the effects on spatial pattern for richness of three and nine classes, and spatial aggregations of $0,0.3$ and 1 . The two figures separate landscapes by class-abundance distributions (evenness), resulting in the subset of six 
landscape plots for both the equal (Fig. 1.5) and geometric (Fig. 1.6) CAD modeled landscapes.

For each of the 24 landscape types with unique characteristics 10 replicates with $1,000 \times 1,000$ cells (total number of cell $=1,000,000$ ) were generated, resulting in 240 neutral landscapes with known properties. Aggregation algorithms were evaluated for four scale factors $-5,9,15$ and $25(25,81,225$, and 625 original grid cells per scaled grid cell, respectively). Origin of the scaled grid was randomized five times for each scale factor and landscape to evaluate effects of the arbitrary origin of scaled grids on class-label fidelity. Applying scale factor and random origin to each of the 240 landscapes resulted in 4,800 datasets of tabulated relative class abundances for each scaled grid cell.

\section{Algorithm Comparison}

Efficacy of the proposed MDGP-scaling algorithm was first evaluated in comparison to two commonly-used, categorical, spatial-aggregation algorithms, the majority rule and the random rule. Majority rule is the simple majority or plurality decision rule, where the assigned output class label is the input class with the highest proportion of sub-samples (mode). Hence, the majority rule can be equated to the simplest case of an $M D G P$-scaling algorithm with a single part or the lowest possible class-label precision of $100 \%$. The random rule assigned the output class at random from the set of sub-samples, which means that class label probability was proportional to class abundance.

Since one of the main concerns with spatial scaling was loss of information content, the three classifiers were compared on the basis of mean information retention at the landscape scale. Information retention was assessed to determine if differences in information retention were significantly higher for the $M D G P$-scaling algorithm across the 
24 landscape types and 4 scale factors that were evaluated in this study. Retention of information content in a landscape was calculated as the mean IR of all classified grid cells. Landscape-scale mean IR was compared for the MDGP-scaling algorithm vs. the majorityand random-rule algorithm IR using pairwise-paired Wilcoxon rank-sign tests (Wilcoxon 1945) because not all compared subsets were normally distributed. Test $p$-values were adjusted using the Bonferroni correction for multiple comparisons by multiplying $p$-values by the number of comparisons. Since there were 10 replicates for each landscape type with five iterations of random origin for each landscape, the number of paired samples for each landscape was $50(N=50)$. Parameters for the MDGP-scaling algorithm for this comparison were class-label precision of $33.3 \%$ (part $=3$ ) and a representativeness threshold of $1 \%$. The total number of scaled landscape models that were evaluated was 14,400 (three algorithms x 4,800 aggregated landscape datasets).

MDGP-Scaling Algorithm Consistency and Sensitivity to Scaling Parameters and Landscape Characteristics

The second objective was to evaluate consistency of the MDGP-scaling algorithm and to assess the effects of landscape characteristics and scaling parameters on IR, class count and class-label fidelity. Consistency of an algorithm is crucial to confidence in the results it delivers. The assumption was that a consistent and reliable algorithm produced similar results when presented with similar data patterns. Consistency in this study was defined as reproducibility of scaling output characteristics across different simulated random landscapes that were congruent in the key characteristics of richness, evenness and spatial aggregation. For upscaling landscapes, it was expected that the scaled landscapes originating at arbitrary grid origins of the same original landscape would produce similar 
scaled data patterns, as reflected in low variability of scaling results, but that variability would increase with scale factor as heterogeneity of grid cells increased.

Three indicators that were expected to display low variance for a consistent algorithm, and thus were suitable for algorithm consistency assessment, were (1) information retention, (2) class count, and (3) class-label fidelity or the consistency in classification schemes. Class-label fidelity (CLF) was defined as (1) the mean probability of class label recurrence across all class labels produced for the five random origins of each landscape $\left(\mathrm{CLF}_{\mathrm{mnPrb}}\right)$ and (2) the proportion of classes for which recurrence probability was one $\left(\mathrm{CLF}_{\mathrm{prp} 1}\right)$. The higher $\mathrm{CLF}_{\mathrm{mnPrb}}$ and $\mathrm{CLF}_{\mathrm{prp} 1}$, the more consistent and reproducible the scaled classification schemes. Consequently, the more stable a classification system was across random origin realizations, the higher the probability that scaled classes were detectable at that scale for continuous landscapes.

Consistency in CLF was evaluated with the variability of mean class probability of label recurrence calculated at the landscape level $(N=5)$ and then summarized with the mean and standard deviation across the 10 simulated iterations of each landscape type. Consistency was then compared across all landscape types by scale factor and class-label precision. Mean information retention and class count consistency were evaluated at the landscape level with the standard deviations calculated across the five scaled random grid origin results $(N=5)$ for each random landscape. Standard deviations were then summarized and compared across all 10 simulated landscapes for the 24 landscape types. For a consistent algorithm, even when scaling results were compared across different simulations of random landscapes that were generated with the same properties (i.e., 
richness, class-abundance distribution and spatial aggregation), the scaled landscapes were expected to display similar properties and to have little variation.

To evaluate robustness to random configuration differences for landscapes with the same characteristics, standard deviations of information retention and class count were also calculated across the five random origins for all 10 simulations of each landscape type ( $N$ $=50)$ and summarized with mean standard deviation across all landscapes. Variance was expected to increase slightly but to stay low if the algorithm was robust to slight differences in landscape configurations that did not differ in the core properties of richness, classabundance distribution or spatial aggregation.

Sensitivity of the MDGP-scaling algorithm to scaling parameters and landscape characteristics was assessed with the magnitude of effects on IR and CLF evaluated by landscape type. Information retention was expected to significantly increase, while CLF was expected to decrease with increasing class-label precision, regardless of landscape type and scale factor. Significance of differences in IR and CLF between class-label precisions was tested with pairwise-paired Wilcoxon rank-sign tests (Wilcoxon 1945), and $p$-values were adjusted using the Bonferroni correction. The magnitude of differences between class-label precisions for both variables, however, was expected to vary with scale factor and landscape characteristics.

Since it was expected that IR increased with class-label precision, but that, simultaneously, CLF decreased, I also investigated the relationships between these variables and the scaled output class count. Predictability in the relationship between IR, class count and CLF establishes the foundation for a formal definition of algorithm optimization such that selection of the class-label precision parameter optimizes IR while 
minimizing scaled class count and maximizing CLF. Consistency and sensitivity of the three indicators (IR, class count and CLF) were assessed for four scale factors $(5,9,15$ and 25) and four class-label precisions, ranging from two to five parts (i.e., 2, 3, 4, and 5 representing class-label precisions of 50,33.3, 25, and $20 \%$, respectively). For the simulation study, the landscape level representativeness threshold was maintained constant at $1 \%$ and class homogeneity at $90 \%$. The total number of scaled landscape models that were evaluated was 24,000 (five class-label precisions x 4,800 aggregated landscape datasets).

The MDGP-scaling algorithm, simulation and test framework, data analysis and visualization was scripted in R ( $\mathrm{R}$ Core Team 2013), making extensive use of packages "raster" (Hijmans and van Etten 2010), "rgdal" (Bivand, Keitt, and Rowlingson 2013), "compositions" (Van Den Boogaart and Tolosana-Delgado 2008), "foreach" and "doParallel" (Revolution Analytics and Weston 2013). Neutral landscape generation and scaled data aggregation for random landscape origins for the different scale factors was scripted in Python 2.7 (Python Software Foundation, https://www.python.org/). The neutral landscapes were generated utilizing the Python module "nlmpy" (Etherington, Holland, and O'Sullivan 2015), which implemented the mid-point displacement algorithm (Fournier, Fussell, and Carpenter 1982; Palmer 1992). All data processing was performed with the high-performance-computing cluster (HPCC) of the Instructional \& Research Computing Center (IRCC) at Florida International University.

\section{RESULTS}

Results of algorithm comparison are reported first, comparing information retention and class-label fidelity of the proposed MDGP-scaling algorithm to those of majority- and 
random-rule algorithms. Then, results for algorithm consistency and sensitivity of the MDGP-scaling algorithm to landscape characteristics, class-label precision and scale factor are presented.

\section{Algorithm Comparison}

A comparison of IR for the three algorithms showed that mean IR was significantly higher and that its standard deviation was significantly lower for MDGP-scaled vs. majority- or random-rule aggregated datasets for all 24 landscape types and for all four scale factors (pairwise-paired Wilcoxon rank-sign tests; Bonferroni adjusted $p<0.001 ; N$ $=50)($ Tbl. 1.3; and Figs. 1.7 for equal CAD models and 1.8 for geometric CAD models). Difference in information loss was consistently less between MDGP and majority-rule vs. random-rule aggregated landscapes (Figs. 1.7 and 1.8, Tbl. 1.3). Differences of mean IR between $M D G P$ and the other two algorithms were greater for more dispersed landscapes, monotonously decreasing as spatial aggregation increased, regardless of richness, classabundance distribution, and scale factor (Figs. 1.7 and 1.8, Tbl. 1.3). Scale factor had a greater effect on IR for majority- and random-rule aggregated landscapes than MDGPscaled landscapes for all landscapes with equal and geometric CAD with spatial aggregation factors greater than zero (Figs. 1.7 and 1.8, Tbl. 1.3). The difference in IR between MDGP-scaled and majority-rule aggregated landscapes increased with scale factor for all landscapes (Figs. 1.7 and 1.8, Tbl. 1.3).

Standard deviation of IR was consistently lower for MDGP-scaled landscapes for all landscapes and scale factors, except for two landscapes with equal class-abundance distribution, when richness was greater than three and spatial aggregation was zero (Fig. 1.7, Tbl. 1.3). On average, standard deviation of mean IR was low for all MDGP-scaled 
landscapes $(0.7 \pm 0.6)$, whereas majority- and random-rule aggregated landscapes, on average, had significantly greater standard deviations ( $1.8 \pm 1.1$ and $2.2 \pm 1.3$, respectively) (pairwise-paired Wilcoxon rank-sign tests; Bonferroni adjusted $p<0.001$ ).

\section{MDGP-Scaling Algorithm Consistency}

Consistency of the MDGP-scaling algorithm was high for all three evaluated parameters (information retention, class count and class-label fidelity). Mean variability of standard deviations of landscape-specific IR evaluated across landscapes ranged from $0.036 \pm 0.046 \%$ to $0.13 \pm 0.08 \%$, increasing with scale factor (Tbl. 1.4). Mean of standard deviations evaluated across landscape types and scale factors increased with class-label precision. Variability in class count ranged from $0.12 \pm 0.23$ to $0.54 \pm 0.45$ classes (Tbl. 1.4). As expected, variability increased for both parameters when evaluating the same parameters at the landscape type level, pooling mean estimates across all simulated landscapes and random origins $(N=50)$. Mean standard deviations for information retention on average increased by $0.64 \pm 0.29 \%$ and that of class count by $0.68 \pm 0.23$ (Tbl 1.4). Consistency in class-label fidelity varied from $0.02 \pm 0.02$ to $0.06 \pm 0.05$ for variability of mean class occurrence evaluated by landscape type $(N=10, S D$ across 10 simulations with mean probability calculated for the scaled landscapes with random origin $(N=5))($ Tbl. 1.4). Consistency in the proportion of classes with probability 1 of recurrence across all compared scaling results was on average only $0.05 \pm 0.02$ lower than the consistency for mean probability (Tbl. 1.4).

\section{MDGP-Scaling Sensitivity - Mean Information Retention}

Evaluating the effects of landscape properties and scaling parameters on mean IR indicates consistent patterns for all landscape characteristics and algorithm parameters that 
were assessed. Concerning landscape properties, mean IR was significantly lower (pairwise-paired Wilcoxon rank-sign test; $p<0.001$ ) for landscapes with equal CAD when compared to those with geometric $\mathrm{CAD}$ with richness and spatial aggregation held constant (plots in Fig. 1.9 for equal CAD vs. 1.10 for geometric CAD models, Tbl. 1.5). Mean IR decreased as richness increased and as spatial aggregation decreased (plots in Figs. 1.9 for equal CAD vs. 1.10 for geometric CAD models, Tbl. 1.5).

Considering the effects of scaling parameters within a landscape type, IR consistently increased with class-label precision within a scale factor, but decreased with increasing scale factor (Figs. 1.9 and 1.10, Tbl. 1.5). The magnitude of gain in IR for increasing class-label precision diminished across all landscapes and for all scale factors (Figs. 1.9 and 1.10, Tbl. 1.6). Largest gains in IR were consistently observed when increasing class-label precision from 1-part to 2-part solutions (majority rule or $100 \%$ to $M D G P-2$ or $50 \%$ precision). The gain in IR with increasing class-label precision diminished as spatial aggregation of a landscape increased, and it increased with richness (Figs. 1.9 and 1.10, Tbl. 1.6).

\section{MDGP-Scaling Sensitivity - Class-Label Fidelity}

Class-label fidelity evaluated with the mean probability of recurring class labels produced across scaling iterations was very high for all landscape types, scale factors and class-label precisions (Figs. 1.11 for equal CAD models and 1.12 for geometric CAD, Tbl. 1.7). Classlabel fidelity generally decreased from lower to higher class-label precisions, for lowrichness landscapes and for high spatial aggregation (Figs. 1.11 and 1.12, Tbl. 1.7). When increasing class-label precision, the largest losses of class-label fidelity were encountered for fully aggregated landscapes, regardless of richness and class-abundance distribution 
(Figs. 1.11 and 1.12, Tbl. 1.8). For landscapes with geometric CAD and low spatial aggregation, class-label fidelity actually increased with class-label precision as richness increased to nine classes and scale factors were high (Figs. 1.11 and 1.12, Tbl. 1.8).

Mean probability of class label recurrence for landscapes with equal CAD ranged from $0.99 \pm 0.02$ for a class-label precision of $50 \%$, decreasing to $0.91 \pm 0.1$ for a precision of $20 \%$ (Fig. 1.11, Tbl. 1.7). For landscapes with geometric CAD, a mean probability reduction of $0.02 \pm 0.05$ was observed when compared to the equal CAD landscape version (plots Fig. 1.11 vs. 1.12, Tbl. 1.7).

MDGP-Scaling Sensitivity - Optimization of Class-Label Precision

Combining the results for information retention, class-label fidelity, and scaled vs. original class-count ratios indicates that for most landscapes and scale factors, class-count ratios and IR increased with class-label precision, while CLF declined (Tbl. 1.9). However, this relationship was not uniform across all landscapes, with most drastic differences in behavior observed for differences in class-abundance distribution of the landscape and for high scale factors. For instance, in the case of landscapes with a scale factor of 15, a nonlinear behavior was observed for the relationship between IR and class-count ratio for landscapes with high richness and low spatial aggregation (Figs. 1.13 and 1.14, left panels). The relationship of IR and class-count ratio varied from a monotonic asymptotic behavior, which was observed for most landscapes, to sigmoid and non-monotonic patterns. In cases of non-monotonic changes (e.g., Fig. 1.14, Tbl. 1.9; richness of six or nine classes and spatial aggregation of 0), a strong increase in IR was achieved with no increase or even with a decrease in scaled class count. Instead of increasing the number of classes, the algorithm generated more precise class-label definitions of class compositions, which led 
to an increase in IR while reducing scaled class count. Considering class-label fidelity, only in the case of landscapes with six classes was label mean probability reduced slightly, whereas in the case of nine classes, CLF did not change significantly or increased (Tbl. 1.9). Optimal class precision decreased with spatial aggregation and was lower for landscapes with geometric CAD (Tbl. 1.9). For landscapes with a spatial aggregation of 1 and low class count, the majority rule (precision 100\%) was preferred, because the gain in IR on average was less than 2\% (Figs. 1.13 and 1.14, Tbl. 1.9).

\section{DISCUSSION}

When aggregating data, information is generalized and, therefore, information is lost. Generalization of fine-scale data is often necessary to support coarser-scale modeling efforts, but the optimal degree of generalization is subjective. Validity of generalized data is application-specific, and the scientific question posed by the researcher is ultimately the deciding factor in determining the data's adequacy. In the context of categorical data scaling, two thresholds are of interest: the minimum level of thematic class precision that is required to maintain enough information to answer the scientific question; and the threshold for a class's representativeness, beyond which it is of no ecological interest at the aggregated scale. The minimum level of class-label precision is the point beyond which generalization reduces the information content to levels where the question of interest can no longer be adequately addressed. Both parameters, precision and representativeness, need to be determined with respect to ecological validity and significance.

All categorical raster maps represent generalizations of real landscapes. The degree of generalization of each map depends on the precision in the spatial and thematic domains of the map. The process of spatial aggregation further generalizes, reducing the 
information content for each aggregated scaled unit and, when integrated across the landscape, reducing information content at the landscape scale. The amount of information lost in a scaled and aggregated map unit is zero when no generalization occurs (a full detailed description of each aggregated unit is provided), but information loss increases rapidly with the reduction of class-label precision. However, commonly used spatial aggregation algorithms neither estimate spatially explicit information loss nor quantify information loss at the aggregated landscape scale. These algorithms also do not provide parameter options that permit control of information loss.

The purpose of my study was to develop a new scaling algorithm that addresses the problem of uncontrolled information loss. The proposed MDGP-scaling algorithm was developed in the context of landscape ecology and integrates concepts of community ecology and phytosociology, acknowledging variability in co-occurrence patterns of species or community classes as spatial scales change (i.e., cell size increases). This research strongly supports the application of the newly developed algorithm to scale categorical landscape representations to lower (coarser) spatial resolutions. The algorithm overcomes the limitations of cluster algorithms for quantitative grouping, which are often employed in the fields of community ecology and phytosociology. The proposed criteria for evaluation of algorithm consistency were location-specific information retention integrated across the landscape, scaled class-count consistency and class-label fidelity. Information retention was introduced as an effective means to compare agreement of categorical data vectors; this metric can also be used to classify samples in a classification system with quantitatively defined classes. Information retention is a true metric and, hence, is also a valid evaluation parameter that facilitates direct comparison of scaling 
results between algorithms or among results for different parameter settings and varying landscape characteristics.

The simulation study built confidence in the scaling properties of the algorithm and the applicability across a large range of landscape settings and spatial data-scaling needs. The algorithm was robust in consistently generating representative class labels while significantly increasing information retention for the scaled landscapes when compared to other commonly used algorithms. Low variability in class-count ratios and class-label fidelity provided the foundation for confidence in reproducibility and reliability of the MDGP-scaling algorithm.

The framework developed here allows expansion to new evaluation parameters. Future inclusion of parameters that address class-proportion consistency and consistency in spatially explicit class-label agreement will further increase confidence in scaling results. More formalized indices and criteria that consider trade-offs among information retention, class-label fidelity, class abundance, and spatially explicit class consistency are needed to implement statistically sound optimization routines. Implementing an integrated tuning method to optimize the selection of the class-label precision parameter can then lead to algorithm-generated recommendations on parameter selection for specific landscapes.

\section{MDGP-Scaling Sensitivity to Landscape Characteristics and Scaling Parameters}

Simulation results suggested that, especially when dealing with less aggregated, patchy landscapes, the MDGP-scaling algorithm was very successful in retaining information at a high level when other algorithms (i.e., majority and random rule) failed to do so. Information loss for an aggregated spatial unit of a scaled map increased drastically with richness of a landscape and with scale factor. Implementing a scaling parameter that 
controls class-label precision effectively reduced information loss of scaled landscapes as class-label precision was increased. Information retention gain was especially high for landscapes with high richness and low to medium spatial aggregation levels, which had high information reduction in landscapes aggregated with majority- and random-rule algorithms.

The MDGP-scaling algorithm generates scale-specific classification systems. The more stable a classification system is, the higher the probability that scaled classes are detectable at that scale at random locations across the continuous landscape. The demonstrated high proportion of classes with recurrence probability of one across random landscapes and the low variance across scaled landscapes with random origins instills high confidence in class-label representation across the larger landscape. Class-label fidelity was generally high even for high label precisions and scale factors but was most sensitive to spatial aggregation of the landscape. A sharp reduction in class-label fidelity with increasing class-label precision was observed for landscapes that were highly aggregated.

Gain in information retention with increasing class-label precision was not linear but rather followed the law of diminishing returns. Richness in scaled classification systems generally increased with class-label precision, and simultaneously, class-label fidelity diminished. Combining the effects of class-label precision on information retention and class-label fidelity it was demonstrated that it was possible to reduce classlabel precision, which in many cases lowered information retention only marginally, while significantly enhancing class-label fidelity and reducing class count.

Reducing class-label precision lead to more general classification schemes, resulting in higher representativeness of a class schema to different realizations of the same 
landscape (random origin) or even realizations of sets of landscapes with similar characteristics. However, it was also demonstrated that an increase in class-label precision does not always increase scaled class count or reduce class-label fidelity. Cases where an increase in class-label precision increased information retention but, unexpectedly, also reduced scaled class count and increased class-label fidelity demonstrate that landscape characteristics can have a strong effect on the scaling results and that the parameter that controls class-label precision needs to be optimized for each specific landscape and scale factor.

\section{Applications of the MDGP-Scaling Algorithm}

The motivation for this study was two-fold: (1) to make improvements in spatially explicit ecological modeling; and (2) to support the application of remote sensing to multiscale mapping. When developing ecological models, a crucial aspect is the scale of analysis; depending on environmental and ecological processes, results can vary significantly when evaluated at different scales (Scheiner et al. 2000). Essential components for the reliable interpretation of results are selecting the appropriate scale of analysis at which ecological processes of interest operate and choosing the required precision of the data.

Spatially explicit maps at high spatial resolutions provide the precision required to separate and distinguish borders of units of the smallest meaningful spatial unit (i.e., land cover or land use, or other categorized landscape domains). These high-resolution data layers contain fine-scale spatially explicit information. Efficient upscaling of these highinformation data layers requires more than just spatial aggregation of discrete spatial units 
of the original classes; it requires classification of landscape objects into meaningful and scale-specific representative classes.

The MDGP-scaling algorithm is the first algorithm that generates data-driven scaleappropriate classification schemes while conducting spatial data aggregation. My study demonstrated that the algorithm consistently delivers representative class descriptors (labels), generating new, scale-specific classification systems. To attain adequate precision in the thematic domain that supports the use of the aggregated product in subsequent ecological models, the algorithm provides a control parameter that allows for optimization of information retention and class-label fidelity in the thematic domain.

\section{Implications for Landscape Ecology Modeling}

Spatial aggregation and scaling of high-resolution maps for use in spatially explicit landscape models that model at lower spatial resolutions requires data upscaling. The effects of $M D G P$-scaling on accuracy and precision of ecological modeling still needs to be demonstrated. However, the consistent gain in information retention of $M D G P$-scaled landscapes and their associated reproducible classification systems strongly suggest that the increased precision of scaled maps will improve ecological models that use these scaled maps when compared to maps that have been scaled with algorithms that do not consider scale-dependent classification systems and do not optimize information retention.

The major advantages of the proposed MDGP-scaling algorithm are the userdefined parameters for class-label precision, which facilitate optimization of scaling results and allow molding these results to the needs of a specific research scope. The ability to also produce spatially explicit and exhaustive layers of information loss can be a valuable input for ecological models that consider the propagation of uncertainty and error. 


\section{Implications for Remote Sensing Applications}

With increasing availability of remotely sensed data, ranging from very high spatial resolutions on the order of centimeters (e.g., airborne sensors mounted on Unmanned Aerial Systems) to low-resolution satellite data with spatial resolutions greater than 1 kilometer (e.g., the Advanced Very High Resolution Radiometer (AVHRR)), there is a need for robust and reliable aggregation methods for categorical data. Detection of landcover change over time will benefit from properly scaled landscapes.

The MDGP-scaling method unifies the classification system with the scaling process while maintaining the highest level of information content possible. I expect that the effects of increased location-specific information content combined with a landscapespecific, representative, classification scheme increases the detectability of the scaled classes from spectral signatures of remotely sensed data.

Scaling high-resolution maps to the spatial resolution of a sensor with lower spatial resolution (e.g., WorldView-2 2x2 m pixels scaled to Landsat 30x30 m pixels) with the purpose of detecting the scaled classes from remotely sensed data of the lower resolution sensor will benefit from high-precision classification schemes that capture frequently recurring co-occurrence patterns at the spatial resolution of the sensor. Such high-precision classification schemes at low spatial resolutions are not easy to establish ad-hoc. The automated establishment of a classification system with quantitative class definitions is expected to increase classification accuracy. The effects of MDGP-scaling algorithm aggregation on the spectral separability of scaled classes from lower resolution remotely sensed reflectance patterns still needs to be demonstrated. 


\section{Implications for Community Ecology and Phytosociology}

In community ecology and phytosociology, it is common practice to establish community classes or to classify co-occurrence patterns through clustering algorithms of samples gathered at a specified resolution (e.g., $1 \mathrm{~m}^{2}$ ) in the field. Natural groupings in data that represent frequently co-occurring patterns rely on cluster algorithm consistency and reproducibility of results. The new MDGP-scaling algorithm can be applied to any multi-dimensional data pattern that is produced by relevés. Future work will focus on the sampling intensities required to generate representative and robust classes valid for the sampled landscape at the sampled scale. This will increase the confidence in applying the algorithm in the field of phytosociology and community ecology, where sample data are the norm and census is the exception. 


\section{TABLES}

Table 1.1. Number of weak compositions for compositions with constraint of exactly $100 \%$ coverage.

\begin{tabular}{c|rrrrrr}
\hline & \multicolumn{7}{|c}{ Scale Factor } \\
Richness & 3 & 5 & 7 & 9 & 15 & 25 \\
\hline $\mathbf{2}$ & 10 & 26 & 50 & 82 & 226 & 626 \\
$\mathbf{3}$ & 55 & 351 & 1,275 & 3,403 & 25,651 & 196,251 \\
$\mathbf{4}$ & 220 & 3,276 & 22,100 & 95,284 & $1,949,476$ & $41,081,876$ \\
$\mathbf{5}$ & 715 & 23,751 & 292,825 & $2,024,785$ & $111,607,501$ & \\
$\mathbf{6}$ & 2,002 & 142,506 & $3,162,510$ & $34,826,302$ & & \\
$\mathbf{7}$ & 5,005 & 736,281 & $28,989,675$ & & & \\
$\mathbf{8}$ & 11,440 & $3,365,856$ & & & & \\
$\mathbf{9}$ & 24,310 & $13,884,156$ & & & & \\
$\mathbf{1 0}$ & 48,620 & $52,451,256$ & & & & \\
\hline Precision & $11.11 \%$ & $4 \%$ & $2.04 \%$ & $1.23 \%$ & $0.44 \%$ & $0.16 \%$
\end{tabular}

Table 1.2. Number of constrained combinations and precision limits for equal-part partitioning of $\boldsymbol{n}$ dimensions (richness).

\begin{tabular}{c|rrrrrr}
\hline & \multicolumn{7}{|c}{ Part (partitions) } \\
Richness & 1 & 2 & 3 & 4 & 5 & 6 \\
\hline \hline $\mathbf{2}$ & 2 & 3 & 4 & 5 & 6 & 7 \\
$\mathbf{3}$ & 3 & 6 & 10 & 15 & 21 & 28 \\
$\mathbf{4}$ & 4 & 10 & 20 & 35 & 56 & 84 \\
$\mathbf{5}$ & 5 & 15 & 35 & 70 & 126 & 210 \\
$\mathbf{6}$ & 6 & 21 & 56 & 126 & 252 & 462 \\
$\mathbf{7}$ & 7 & 28 & 84 & 210 & 462 & 924 \\
$\mathbf{8}$ & 8 & 36 & 120 & 330 & 792 & 1716 \\
$\mathbf{9}$ & 9 & 45 & 165 & 495 & 1287 & 3003 \\
$\mathbf{1 0}$ & 10 & 55 & 220 & 715 & 2002 & 5005 \\
\hline Precision & $100 \%$ & $50 \%$ & $33.33 \%$ & $25 \%$ & $20 \%$ & $16.67 \%$
\end{tabular}


Table 1.3. Mean information retention (IR) \pm standard deviations across landscape iterations and random origin $(N=50)$ for randomrule (RND), majority-rule (MAJ-1) and MDGP-scaling algorithm with label precision of 33\% (MDGP-3). CAD = Class-Abundance Distribution; rch = Richness; sptAgg. = Spatial Aggregation. Pairwise-paired Wilcoxon rank-sign tests indicate that for all landscapes and scale factors (SF), mean IR for MDGP-scaling was significantly greater and standard deviation of information retention was significantly lower for MDGP-scaled landscapes when compared to majority- and random-rule scaled landscapes.

\begin{tabular}{|c|c|c|c|c|c|c|c|c|c|c|c|c|c|c|}
\hline \multirow{2}{*}{\multicolumn{3}{|c|}{ CAD rch sptAgg }} & \multicolumn{3}{|c|}{ SF - 5} & \multicolumn{3}{|c|}{ SF - 9} & \multicolumn{3}{|c|}{ SF - 15} & \multicolumn{3}{|c|}{ SF - 25} \\
\hline & & & RND & MAJ-1 & MDGP-3 & RND & MAJ-1 & MDGP-3 & RND & MAJ-1 & MDGP-3 & RND & MAJ-1 & MDGP-3 \\
\hline \multirow{12}{*}{ equal } & \multirow{4}{*}{3} & 0 & $57.6 \pm 1.6$ & $66.4 \pm 1.4$ & $89.5 \pm 0.3$ & $52.8 \pm 1.7$ & $62.5 \pm 1.6$ & $88.3 \pm 0.2$ & $49.9 \pm 1.8$ & $59.8 \pm 1.7$ & $88 \pm 0.1$ & $47.3 \pm 1.9$ & $57.3 \pm 1.9$ & $88 \pm 0.3$ \\
\hline & & 0.3 & $83.1 \pm 3.7$ & $87.7 \pm 2.7$ & $95.3 \pm 1$ & $79.3 \pm 4.4$ & $85.2 \pm 3.3$ & $94.3 \pm 1.1$ & $76.1 \pm 5$ & $82.7 \pm 3.9$ & $93.5 \pm 1.1$ & $72.4 \pm 5.5$ & $79.9 \pm 4$ & $92.7 \pm 1.1$ \\
\hline & & 0.6 & $94.6 \pm 1.6$ & $96.1 \pm 1.2$ & $98.5 \pm 0.5$ & $92.4 \pm 2.2$ & $94.6 \pm 1.6$ & $97.9 \pm 0.6$ & $89.9 \pm 3$ & $92.8 \pm 2.1$ & $97.1 \pm 0.8$ & $86.5 \pm 4$ & $90.4 \pm 2.9$ & $96.2 \pm 1.1$ \\
\hline & & 1 & $98.6 \pm 0.4$ & $99 \pm 0.3$ & $99.2 \pm 0.1$ & $97.7 \pm 0.6$ & $98.3 \pm 0.4$ & $99.2 \pm 0.1$ & $96.3 \pm 0.9$ & $97.4 \pm 0.7$ & $99 \pm 0.2$ & $94.3 \pm 1.4$ & $95.9 \pm 1$ & $98.4 \pm 0.4$ \\
\hline & \multirow{4}{*}{6} & 0 & $35.4 \pm 1.5$ & $45.2 \pm 1.4$ & $80.6 \pm 1$ & $30.5 \pm 1.5$ & $40.2 \pm 1.5$ & $78 \pm 1.6$ & $28.1 \pm 1.6$ & $37.2 \pm 1.6$ & $75.4 \pm 1.9$ & $26 \pm 1.5$ & $34.8 \pm 1.7$ & $72.9 \pm 2.1$ \\
\hline & & 0.3 & $60.1 \pm 3.9$ & $68.9 \pm 3.6$ & $89.9 \pm 1$ & $53.8 \pm 4.1$ & $63.6 \pm 3.9$ & $88 \pm 1.2$ & $49.1 \pm 4$ & $59 \pm 4$ & $86.5 \pm 1.2$ & $44.6 \pm 3.9$ & $54.5 \pm 4$ & $85.1 \pm 1.1$ \\
\hline & & 0.6 & $89.5 \pm 1.8$ & $92.4 \pm 1.3$ & $96.9 \pm 0.5$ & $85.4 \pm 2.5$ & $89.6 \pm 1.8$ & $95.9 \pm 0.7$ & $80.7 \pm 3.2$ & $86.3 \pm 2.3$ & $94.6 \pm 0.8$ & $74.8 \pm$ & $81.8 \pm 3$ & $93.1 \pm 1$ \\
\hline & & 1 & $96.9 \pm 0.7$ & $97.8 \pm 0.5$ & $98.2 \pm 0.3$ & $94.7 \pm 1.2$ & $96.2 \pm 0.9$ & $98 \pm 0.1$ & $91.7 \pm 1.8$ & $94 \pm 1.3$ & $97.5 \pm 0.4$ & $86.9 \pm 2.9$ & $90.6 \pm 2$ & $96.4 \pm 0.7$ \\
\hline & \multirow{4}{*}{9} & 0 & $26.5 \pm 1.3$ & $36.1 \pm 1.3$ & $70.6 \pm 1.6$ & $21.9 \pm 1.3$ & $30.9 \pm 1.4$ & $64.5 \pm 2.2$ & $|19.7 \pm 1.4|$ & $27.9 \pm 1.5$ & $60.5 \pm 2.4$ & $18 \pm 1.4$ & $25.7 \pm 1.6$ & \pm 2.6 \\
\hline & & 0.3 & $52.4 \pm 3.2$ & $61.9 \pm 3.2$ & $87.7 \pm 1$ & $45.9 \pm 3.1$ & $56 \pm 3.3$ & $85.6 \pm 1$ & $41.4 \pm 3$ & $51.4 \pm 3.3$ & $84 \pm 1$ & $37.1 \pm 2.8$ & $46.8 \pm 3.2$ & $82.1 \pm 1.6$ \\
\hline & & 0.6 & $83.6 \pm 3.9$ & $88.1 \pm 2.9$ & $95.2 \pm 1$ & $77.3 \pm 5.1$ & $83.7 \pm 3.9$ & $93.7 \pm 1.3$ & $70.9 \pm 6$ & $78.6 \pm 4.9$ & $92.1 \pm 1.5$ & $63.4 \pm 6.6$ & $72.3 \pm 5.7$ & $90.3 \pm 1.7$ \\
\hline & & 1 & $95 \pm 0.9$ & $96.4 \pm 0.7$ & $97.1 \pm 0.3$ & $91.4 \pm 1.5$ & $93.9 \pm 1.1$ & $96.9 \pm 0.2$ & $86.6 \pm 2.4$ & $90.4 \pm 1.7$ & $96.1 \pm 0.5$ & $79.2 \pm 3.7$ & $84.9 \pm 2.7$ & $94.4 \pm 0.9$ \\
\hline \multirow{12}{*}{ geom } & \multirow{4}{*}{3} & 0 & $64.2 \pm 1.3$ & $72.1 \pm 1.1$ & $90.9 \pm 0.3$ & $60 \pm 1.4$ & $69 \pm 1.2$ & $89.7 \pm 0.3$ & $57.6 \pm 1.5$ & $66.9 \pm 1.2$ & $89 \pm 0.2$ & $55.4 \pm 1.6$ & $65.2 \pm 1.3$ & $88.8 \pm 0.2$ \\
\hline & & 0.3 & $85.2 \pm 2.2$ & $89.2 \pm 1.6$ & $95.9 \pm 0.6$ & $81.9 \pm 2.6$ & $87.1 \pm 1.9$ & $95 \pm 0.6$ & $79 \pm 2.9$ & $84.9 \pm 2.3$ & $94.2 \pm 0.6$ & $75.8 \pm 3.4$ & $82.5 \pm 2.7$ & $93.5 \pm 0.7$ \\
\hline & & 0.6 & $95.9 \pm 1.1$ & $97 \pm 0.8$ & $98.8 \pm 0.3$ & $94.2 \pm 1.5$ & $95.9 \pm 1.1$ & $98.4 \pm 0.4$ & $92.3 \pm 2$ & $94.6 \pm 1.4$ & $97.8 \pm 0.5$ & $89.8 \pm 2.5$ & $92.8 \pm 1.9$ & $97.2 \pm 0.7$ \\
\hline & & 1 & $98.9 \pm 0.3$ & $99.2 \pm 0.3$ & $99.3 \pm 0.1$ & $98 \pm 0.6$ & $98.6 \pm 0.4$ & $99.3 \pm 0.1$ & $96.9 \pm 0.9$ & $97.8 \pm 0.6$ & $99 \pm 0.2$ & $95.2 \pm 1.4$ & $96.5 \pm 1$ & $98.7 \pm 0.4$ \\
\hline & \multirow{4}{*}{6} & & $52.1 \pm 1.3$ & $61 \pm 1.1$ & $86.1 \pm 0.6$ & $47.8 \pm 1.3$ & $57.8 \pm 1$ & $84.9 \pm 0.7$ & $45.6 \pm 1.3$ & $56 \pm 0.9$ & $84 \pm 0.7$ & $43.7 \pm 1.4$ & $54.7 \pm 0.9$ & $83.1 \pm 0.8$ \\
\hline & & 0.3 & $76.7 \pm 2.3$ & $82.6 \pm 1.9$ & $93.7 \pm 0.5$ & $72.2 \pm 2.5$ & $79.4 \pm 2.1$ & $92.5 \pm 0.6$ & $68.7 \pm 2.6$ & $76.4 \pm 2.3$ & $91.5 \pm 0.7$ & $65 \pm 2.6$ & $73.2 \pm 2.5$ & $90.5 \pm 0.7$ \\
\hline & & 0.6 & $92.4 \pm 1.3$ & $94.5 \pm 0.9$ & $97.5 \pm 0.3$ & $89.4 \pm 1.7$ & $92.5 \pm 1.3$ & $96.7 \pm 0.4$ & $86 \pm 2.3$ & $90 \pm 1.7$ & $95.9 \pm 0.5$ & $81.6 \pm 2.8$ & $86.8 \pm 2.1$ & $94.7 \pm 0.6$ \\
\hline & & 1 & $98.2 \pm 0.6$ & $98.7 \pm 0.4$ & $98.8 \pm 0.2$ & $96.9 \pm 1$ & $97.8 \pm 0.7$ & $98.5 \pm 0.2$ & $95.2 \pm 1.5$ & $96.6 \pm 1.1$ & $98.2 \pm 0.3$ & $92.6 \pm 2.3$ & $94.7 \pm 1.7$ & $97.6 \pm 0.6$ \\
\hline & \multirow{4}{*}{9} & 0 & $51.4 \pm 1$ & $60.4 \pm 0.8$ & $85.1 \pm 0.4$ & $47.3 \pm 1.1$ & $57.3 \pm 0.9$ & $83.8 \pm 0.5$ & $45.2 \pm 1.2$ & $55.6 \pm 0.8$ & $82.9 \pm 0.5$ & $43.1 \pm 1.2$ & $54.3 \pm 0.8$ & $82.2 \pm 0.5$ \\
\hline & & 0.3 & $72.8 \pm 3.4$ & $79.1 \pm 2.8$ & $92.4 \pm 0.9$ & $68.2 \pm 3.7$ & $75.6 \pm 3.2$ & $91.2 \pm 1$ & $64.7 \pm 4$ & $72.6 \pm 3.4$ & $90.2 \pm 1.1$ & $61.2 \pm 4.1$ & $69.6 \pm 3.6$ & $88.9 \pm 1.2$ \\
\hline & & 0.6 & $91.3 \pm 1$ & $93.7 \pm 0.7$ & $96.9 \pm 0.3$ & $88 \pm 1.4$ & $91.4 \pm 1$ & $96 \pm 0.4$ & $84.5 \pm 1.8$ & $88.8 \pm 1.3$ & $95.1 \pm 0.5$ & $80 \pm 2.2$ & $85.4 \pm 1.6$ & $94.1 \pm 0.6$ \\
\hline & & 1 & $97.1 \pm 0.7$ & $97.9 \pm 0.5$ & $98.3 \pm 0.3$ & $95 \pm 1.1$ & $96.4 \pm 0.8$ & $97.9 \pm 0.3$ & $92.3 \pm 1.6$ & $94.4 \pm 1.2$ & $97.1 \pm 0.4$ & $88.3 \pm 2.3$ & $91.5 \pm 1.7$ & $96.1 \pm 0.5$ \\
\hline
\end{tabular}


Table 1.4. Consistency of the MDGP-scaling algorithm assessed for four levels of class-label precision (MDGP-2 to 5) and four scale factors (SF). Indicator parameters were standard deviation of information retention (in percent) and class count evaluated by landscape $(N=$ 240) and landscape type $(N=24)$. Indicator parameters for class-label fidelity were mean probability of class occurrence (Mean Prb.) and proportion of classes with recurrence probability of 1 (Prp. of Prb.1). Both indictors were calculated across the five random origins of each landscape. Standard deviation of class-label fidelity was evaluated by individual landscape $(N=10)$ and results were summarized by landscape type $(N=24)$.

\begin{tabular}{|c|c|c|c|c|c|c|c|c|c|c|}
\hline & & & SF & & SF & & SF - & & SF & \\
\hline & & & Mean & SD & Mean & SD & Mean & SD & Mean & SD \\
\hline & & MDGP-2 & 0.036 & 0.046 & 0.045 & 0.032 & 0.077 & 0.049 & 0.126 & 0.073 \\
\hline & Inforn & MDGP-3 & 0.036 & 0.041 & 0.054 & 0.047 & 0.070 & 0.055 & 0.109 & 0.077 \\
\hline & Reter & MDGP-4 & 0.047 & 0.050 & 0.061 & 0.059 & 0.068 & 0.045 & 0.103 & 0.078 \\
\hline 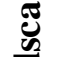 & & MDGP-5 & 0.053 & 0.054 & 0.059 & 0.055 & 0.072 & 0.060 & 0.094 & 0.073 \\
\hline 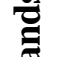 & & MDGP-2 & 0.177 & 0.271 & 0.117 & 0.229 & 0.141 & 0.279 & 0.176 & 0.320 \\
\hline & Class Count & MDGP-3 & 0.214 & 0.284 & 0.257 & 0.329 & 0.251 & 0.355 & 0.328 & 0.360 \\
\hline & (SD) & MDGP-4 & 0.356 & 0.348 & 0.394 & 0.383 & 0.384 & 0.394 & 0.499 & 0.434 \\
\hline & & MDGP-5 & 0.409 & 0.370 & 0.457 & 0.394 & 0.541 & 0.445 & 0.606 & 0.465 \\
\hline & & MDGP-2 & 0.782 & 0.503 & 0.973 & 0.618 & 1.197 & 0.710 & 1.422 & 0.820 \\
\hline & Imior & MDGP-3 & 0.558 & 0.377 & 0.660 & 0.524 & 0.761 & 0.566 & 0.909 & 0.582 \\
\hline & & MDGP-4 & 0.418 & 0.260 & 0.513 & 0.388 & 0.598 & 0.468 & 0.732 & 0.503 \\
\hline & & MDGP-5 & 0.348 & 0.216 & 0.388 & 0.270 & 0.453 & 0.378 & 0.565 & 0.424 \\
\hline & & MDGP-2 & 0.722 & 0.505 & 0.588 & 0.498 & 0.461 & 0.427 & 0.465 & 0.479 \\
\hline & Class Count & MDGP-3 & 0.846 & 0.625 & 1.066 & 1.070 & 0.913 & 0.775 & 0.883 & 0.652 \\
\hline$E$ & (SD) & MDGP-4 & 1.229 & 0.890 & 1.285 & 0.967 & 1.173 & 0.870 & 0.975 & 0.619 \\
\hline$\stackrel{\Xi}{=}$ & & MDGP-5 & 1.261 & 0.787 & 1.473 & 0.850 & 1.540 & 1.031 & 1.365 & 0.866 \\
\hline g & Class Label & MDGP-2 & 0.031 & 0.027 & 0.019 & 0.023 & 0.023 & 0.028 & 0.027 & 0.030 \\
\hline $\bar{\xi}$ & Fidelity - & MDGP-3 & 0.043 & 0.033 & 0.038 & 0.033 & 0.037 & 0.027 & 0.043 & 0.029 \\
\hline$\stackrel{乛}{=}$ & Mean Prb. & MDGP-4 & 0.047 & 0.036 & 0.041 & 0.031 & 0.043 & 0.035 & 0.046 & 0.020 \\
\hline & (SD) & MDGP-5 & 0.063 & 0.049 & 0.046 & 0.027 & 0.051 & 0.037 & 0.052 & 0.026 \\
\hline & Class Label & MDGP-2 & 0.054 & 0.041 & 0.036 & 0.042 & 0.042 & 0.043 & 0.051 & 0.048 \\
\hline & Fidelity - & MDGP-3 & 0.079 & 0.064 & 0.072 & 0.062 & 0.070 & 0.052 & 0.082 & 0.042 \\
\hline & Prp. of Prb. 1 & MDGP-4 & 0.084 & 0.060 & 0.075 & 0.054 & 0.074 & 0.060 & 0.079 & 0.042 \\
\hline & (SD) & MDGP-5 & 0.106 & 0.081 & 0.080 & 0.043 & 0.087 & 0.054 & 0.096 & 0.053 \\
\hline
\end{tabular}


Table 1.5. Effects of class-label precision and scale factor (SF) on information retention for 24 landscape types varying in classabundance distribution (CAD), richness (rch) and spatial aggregation (sptAgg). Class-label precisions of 50, 33, 25, and 20 percent correspond to $M D G P-2,3,4$ and 5 , respectively.

\begin{tabular}{|c|c|c|c|c|c|c|c|c|c|c|c|c|c|c|c|c|c|c|}
\hline \multirow[b]{2}{*}{ CAD } & \multirow[b]{2}{*}{ rch } & \multirow[b]{2}{*}{ sptAgg } & \multicolumn{4}{|c|}{ SF 5} & \multicolumn{4}{|c|}{ SF 9} & \multicolumn{4}{|c|}{ SF 15} & \multicolumn{4}{|c|}{ SF 25} \\
\hline & & & 50 & 33 & 25 & 20 & 50 & 33 & 25 & 20 & 50 & 33 & 25 & 20 & 50 & 33 & 25 & 20 \\
\hline \multirow{12}{*}{ equal } & \multirow{4}{*}{3} & 0 & 85.4 & 89.5 & 92.2 & 93.7 & 84.2 & 88.3 & 91.4 & 93.0 & $\overline{882.9}$ & 88.0 & 90.7 & 92.8 & 81.6 & $\overline{88.0}$ & $\overline{899.9}$ & 92.8 \\
\hline & & 0.3 & 93.3 & 95.3 & 96.4 & 97.2 & 91.8 & 94.3 & 95.7 & 96.5 & 90.6 & 93.5 & 95.0 & 95.9 & 89.4 & 92.7 & 94.4 & 95.4 \\
\hline & & 0.6 & 97.8 & 98.5 & 98.8 & 99.0 & 97.0 & 97.9 & 98.3 & 98.6 & 96.0 & 97.1 & 97.8 & 98.2 & 94.7 & 96.2 & 97.1 & 97.6 \\
\hline & & 1 & 99.2 & 99.2 & 99.2 & 99.3 & 99.0 & 99.2 & 99.2 & 99.2 & 98.6 & 99.0 & 99.1 & 99.2 & 97.8 & 98.4 & 98.8 & 99.0 \\
\hline & \multirow{4}{*}{6} & 0 & 68.2 & 80.6 & 85.4 & 88.1 & 62.7 & 78.0 & 85.0 & 87.2 & 59.4 & 75.4 & 84.7 & 86.9 & 56.8 & 72.9 & 83.8 & 87.1 \\
\hline & & 0.3 & 85.6 & 89.9 & 92.2 & 93.7 & 83.7 & 88.0 & 90.8 & 92.4 & 81.4 & 86.5 & 89.6 & 91.4 & 78.1 & 85.1 & 88.3 & 90.4 \\
\hline & & 0.6 & 95.7 & 96.9 & 97.5 & 97.9 & 94.1 & 95.9 & 96.7 & 97.3 & 92.3 & 94.6 & 95.8 & 96.5 & 90.1 & 93.1 & 94.7 & 95.6 \\
\hline & & 1 & 98.1 & 98.2 & 98.2 & 98.3 & 97.7 & 98.0 & 98.1 & 98.1 & 96.7 & 97.5 & 97.9 & 98.0 & 94.9 & 96.4 & 97.1 & 97.5 \\
\hline & \multirow{4}{*}{9} & 0 & 56.8 & 70.6 & 78.4 & 82.7 & 50.1 & 64.5 & 75.1 & 81.5 & 46.2 & 60.5 & 71.9 & 79.8 & 43.3 & 57.4 & 69.1 & 77.8 \\
\hline & & 0.3 & 82.7 & 87.7 & 90.3 & 92.0 & 79.8 & 85.6 & 88.7 & 90.7 & 76.3 & 84.0 & 87.3 & 89.4 & 71.8 & 82.1 & 85.9 & 88.1 \\
\hline & & 0.6 & 93.3 & 95.2 & 96.0 & 96.6 & 91.1 & 93.7 & 95.0 & 95.7 & 88.8 & 92.1 & 93.8 & 94.7 & 86.2 & 90.3 & 92.3 & 93.5 \\
\hline & & 1 & 97.1 & 97.1 & 97.2 & 97.3 & 96.4 & 96.9 & 97.0 & 97.0 & 94.7 & 96.1 & 96.5 & 96.8 & 92.0 & 94.4 & 95.5 & 96.0 \\
\hline \multirow{12}{*}{ geom } & \multirow{4}{*}{3} & 0 & 87.3 & 90.9 & 93.1 & 94.5 & 86.2 & 89.7 & 92.4 & 93.7 & 85.0 & 89.0 & 91.7 & 93.4 & 83.7 & 88.8 & 91.0 & 93.2 \\
\hline & & 0.3 & 94.1 & 95.9 & 96.8 & 97.5 & 92.8 & 95.0 & 96.1 & 96.8 & 91.6 & 94.2 & 95.6 & 96.4 & 90.6 & 93.5 & 95.0 & 95.9 \\
\hline & & 0.6 & 98.3 & 98.8 & 99.0 & 99.1 & 97.7 & 98.4 & 98.7 & 98.9 & 96.9 & 97.8 & 98.3 & 98.6 & 95.9 & 97.2 & 97.8 & 98.2 \\
\hline & & 1 & 99.3 & 99.3 & 99.3 & 99.4 & 99.1 & 99.3 & 99.2 & 99.3 & 98.7 & 99.0 & 99.1 & 99.2 & 98.1 & 98.7 & 98.9 & 99.0 \\
\hline & & 0 & 78.9 & 86.1 & 89.1 & 91.2 & 75.9 & 84.9 & 88.0 & 90.3 & 74.1 & 84.0 & 87.1 & 89.6 & 72.8 & 83.1 & 86.4 & 88.8 \\
\hline & & 0.3 & 91.0 & 93.7 & 95.0 & 95.8 & 89.5 & 92.5 & 94.1 & 95.1 & 88.3 & 91.5 & 93.4 & 94.5 & 86.9 & 90.5 & 92.5 & 93.7 \\
\hline & & 0.6 & 96.7 & 97.5 & 97.8 & 98.1 & 95.6 & 96.7 & 97.3 & 97.6 & 94.4 & 95.9 & 96.7 & 97.1 & 92.7 & 94.7 & 95.7 & 96.4 \\
\hline & & 1 & 98.8 & 98.8 & 98.8 & 98.9 & 98.4 & 98.5 & 98.5 & 98.5 & 97.8 & 98.2 & 98.3 & 98.3 & 96.9 & 97.6 & 97.8 & 97.9 \\
\hline & \multirow{4}{*}{9} & 0 & 77.8 & 85.1 & 88.4 & 90.5 & 75.0 & 83.8 & 87.1 & 89.6 & 73.3 & 82.9 & 86.1 & 88.8 & 72.2 & 82.2 & 85.5 & 88.0 \\
\hline & & 0.3 & 89.4 & 92.4 & 93.9 & 94.9 & 87.6 & 91.2 & 93.1 & 94.2 & 86.1 & 90.2 & 92.3 & 93.5 & 84.3 & 88.9 & 91.3 & 92.8 \\
\hline & & 0.6 & 96.1 & 96.9 & 97.3 & 97.5 & 94.8 & 96.0 & 96.6 & 97.0 & 93.5 & 95.1 & 96.0 & 96.5 & 91.9 & 94.1 & 95.2 & 95.8 \\
\hline & & 1 & 98.2 & 98.3 & 98.3 & 98.3 & 97.6 & 97.9 & 97.9 & 97.8 & 96.5 & 97.1 & 97.4 & 97.5 & 95.0 & 96.1 & 96.6 & 96.8 \\
\hline
\end{tabular}


Table 1.6. Differences in mean information retention (IR) as class-label precision increases (rows within scale factors (SF)) for 24 landscape types varying in class-abundance distribution (CAD), richness (rch) and spatial aggregation (sptAgg). Initial reference for $M D G P$-scaling algorithm of two parts (precision $=\mathbf{5 0 \%}$ ) was majority-rule (precision $=\mathbf{1 0 0 \%}$ ). Mean IR increase diminished with increasing class-label precision by landscape type and scale factor (Mean; $N=50$ ). Largest gain was always observed for increase from 1-part to 2-part solutions (majority-rule $100 \%$ to $M D G P-2$ or $50 \%$ ). Class-label precisions of 50, 33, 25 , and 20 percent correspond to MDGP-2, 3, 4 and 5, respectively. Colors indicate classified IR change as class-label precision increases from 100 to 50 , 33, 25 and 20 percent.

\begin{tabular}{|c|c|c|c|c|c|c|c|c|c|c|c|c|c|c|c|c|c|c|}
\hline \multirow[b]{2}{*}{ CAD } & \multirow[b]{2}{*}{ rch } & \multirow[b]{2}{*}{ sptAgg } & \multicolumn{4}{|c|}{ SF 5} & \multicolumn{4}{|c|}{ SF 9} & \multicolumn{4}{|c|}{ SF 15} & \multicolumn{4}{|c|}{ SF 25} \\
\hline & & & 50 & 33 & 25 & 20 & 50 & 33 & 25 & 20 & 50 & 33 & 25 & 20 & 50 & 33 & 25 & 20 \\
\hline \multirow{12}{*}{ equal } & \multirow{4}{*}{3} & $\begin{array}{l}0 \\
0\end{array}$ & 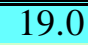 & 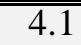 & 2.7 & \begin{tabular}{c|}
1.6 \\
\end{tabular} & 221.7 & $\bar{~} 4.2$ & 3.1 & 101.6 & 23.2 & $\bar{~} 5.0$ & 2.7 & 2.1 & 24.3 & 6.4 & $\bar{~} 1.9$ & 2.8 \\
\hline & & 0.3 & 5.6 & 2.1 & 1.1 & 0.8 & 6.6 & 2.5 & 1.3 & 0.8 & 7.8 & 3.0 & 1.5 & 0.9 & 9.5 & 3.4 & 1.6 & 1.0 \\
\hline & & 0.6 & 1.7 & 0.7 & 0.3 & 0.2 & 2.3 & 0.9 & 0.5 & 0.3 & 3.1 & 1.2 & 0.7 & 0.4 & 4.3 & 1.6 & 0.8 & 0.5 \\
\hline & & 1 & 0.2 & 0.0 & 0.0 & 0.0 & 0.7 & 0.1 & 0.0 & 0.0 & 1.2 & 0.4 & 0.2 & 0.1 & 1.9 & 0.7 & 0.3 & 0.2 \\
\hline & \multirow{4}{*}{6} & 0 & 23.0 & 12.4 & 4.9 & 2.7 & 22.5 & 15.3 & 7.0 & 2.2 & 22.2 & 16.0 & 9.3 & 2.2 & 22.0 & 16.2 & 10.9 & 3.3 \\
\hline & & 0.3 & 16.7 & 4.3 & 2.3 & 1.5 & 20.1 & 4.3 & 2.8 & 1.6 & 22.4 & 5.0 & 3.2 & 1.8 & 23.6 & 6.9 & 3.2 & 2.1 \\
\hline & & 0.6 & 3.3 & 1.2 & 0.6 & 0.3 & 4.5 & 1.7 & 0.9 & 0.5 & 6.1 & 2.3 & 1.2 & 0.7 & 8.3 & 3.0 & 1.5 & 1.0 \\
\hline & & 1 & 0.4 & 0.0 & 0.0 & 0.1 & 1.4 & 0.4 & 0.0 & 0.0 & 2.7 & 0.8 & 0.4 & 0.1 & 4.3 & 1.5 & 0.7 & 0.4 \\
\hline & \multirow{4}{*}{9} & $\overline{0}$ & 20.7 & 13.9 & 7.8 & 4.3 & 19.2 & 14.4 & 10.6 & 6.4 & 18.3 & 14.3 & 11.4 & 7.9 & 17.6 & 14.1 & 11.7 & 8.8 \\
\hline & & 0.3 & 20.8 & 5.1 & 2.5 & 1.7 & 23.8 & 5.8 & 3.1 & 2.0 & 24.9 & 7.7 & 3.3 & 2.1 & 24.9 & 10.3 & 3.8 & 2.2 \\
\hline & & 0.6 & 5.3 & 1.8 & 0.8 & 0.6 & 7.4 & 2.7 & 1.3 & 0.7 & 10.2 & 3.4 & 1.7 & 0.9 & 13.9 & 4.1 & 2.0 & 1.2 \\
\hline & & 1 & 0.7 & 0.1 & 0.1 & 0.1 & 2.5 & 0.6 & 0.0 & 0.0 & 4.3 & 1.4 & 0.5 & 0.3 & 7.1 & 2.3 & 1.1 & 0.5 \\
\hline \multirow{12}{*}{ geom } & \multirow{4}{*}{3} & 0 & 15.3 & 3.5 & 2.3 & 1.3 & 17.2 & 3.5 & 2.7 & 1.4 & 18.1 & 4.1 & 2.7 & 1.6 & 18.5 & 5.2 & 2.2 & 2.1 \\
\hline & & 0.3 & 4.8 & 1.8 & 1.0 & 0.7 & 5.7 & 2.2 & 1.2 & 0.7 & 6.7 & 2.6 & 1.3 & 0.8 & 8.1 & 3.0 & 1.5 & 0.9 \\
\hline & & 0.6 & 1.3 & 0.5 & 0.2 & 0.1 & 1.8 & 0.7 & 0.3 & 0.2 & 2.4 & 0.9 & 0.5 & 0.3 & 3.2 & 1.2 & 0.6 & 0.4 \\
\hline & & 1 & 0.1 & 0.1 & 0.0 & 0.0 & 0.5 & 0.2 & 0.0 & 0.0 & 1.0 & 0.3 & 0.1 & 0.0 & 1.6 & 0.6 & 0.2 & 0.1 \\
\hline & \multirow{4}{*}{6} & 0 & 17.9 & 7.2 & 3.0 & 2.1 & 18.1 & 8.9 & 3.2 & 2.2 & 18.1 & 9.8 & 3.1 & 2.4 & 18.1 & 10.3 & 3.3 & 2.3 \\
\hline & & 0.3 & 8.4 & 2.7 & 1.4 & 0.8 & 10.1 & 3.0 & 1.7 & 0.9 & 11.9 & 3.2 & 1.9 & 1.0 & 13.7 & 3.6 & 2.0 & 1.2 \\
\hline & & 0.6 & 2.2 & 0.8 & 0.4 & 0.3 & 3.1 & 1.1 & 0.6 & 0.3 & 4.3 & 1.6 & 0.7 & 0.4 & 6.0 & 2.0 & 1.0 & 0.7 \\
\hline & & 1 & 0.1 & 0.0 & 0.0 & 0.0 & 0.6 & 0.1 & 0.0 & 0.0 & 1.3 & 0.3 & 0.1 & 0.1 & 2.2 & 0.7 & 0.3 & 0.1 \\
\hline & \multirow{4}{*}{9} & 0 & 17.4 & 7.3 & 3.3 & 2.2 & 17.7 & 8.8 & 3.3 & 2.5 & 17.7 & 9.6 & 3.2 & 2.7 & 17.9 & 10.1 & 3.2 & 2.5 \\
\hline & & 0.3 & 10.2 & 3.1 & 1.5 & 1.0 & 12.0 & 3.6 & 1.9 & 1.1 & 13.4 & 4.1 & 2.2 & 1.2 & 14.7 & 4.6 & 2.4 & 1.5 \\
\hline & & 0.6 & 2.4 & 0.9 & 0.4 & 0.2 & 3.4 & 1.2 & 0.6 & 0.4 & 4.7 & 1.6 & 0.9 & 0.5 & 6.6 & 2.1 & 1.1 & 0.6 \\
\hline & & 1 & 0.4 & 0.1 & 0.0 & 0.0 & 1.2 & 0.3 & 0.0 & 0.1 & 2.1 & 0.6 & 0.2 & 0.1 & 3.5 & 1.1 & 0.5 & 0.2 \\
\hline
\end{tabular}

Parameter Change

$$
\begin{array}{|l|l|l|l|l|l|l|}
>20 & 15-20 & 10-15 & 5-10 & 2-5 & 0-2 & <0 \\
\hline
\end{array}
$$


Table 1.7. Effects of class-label precision (rows within SF) and scale factor (SF) on class-label fidelity for 24 landscape types varying in class-abundance distribution (CAD), richness (rch) and spatial aggregation (sptAgg). Class-label precisions of 50, 33, 25, and 20 percent correspond to $M D G P-2,3,4$ and 5 , respectively.

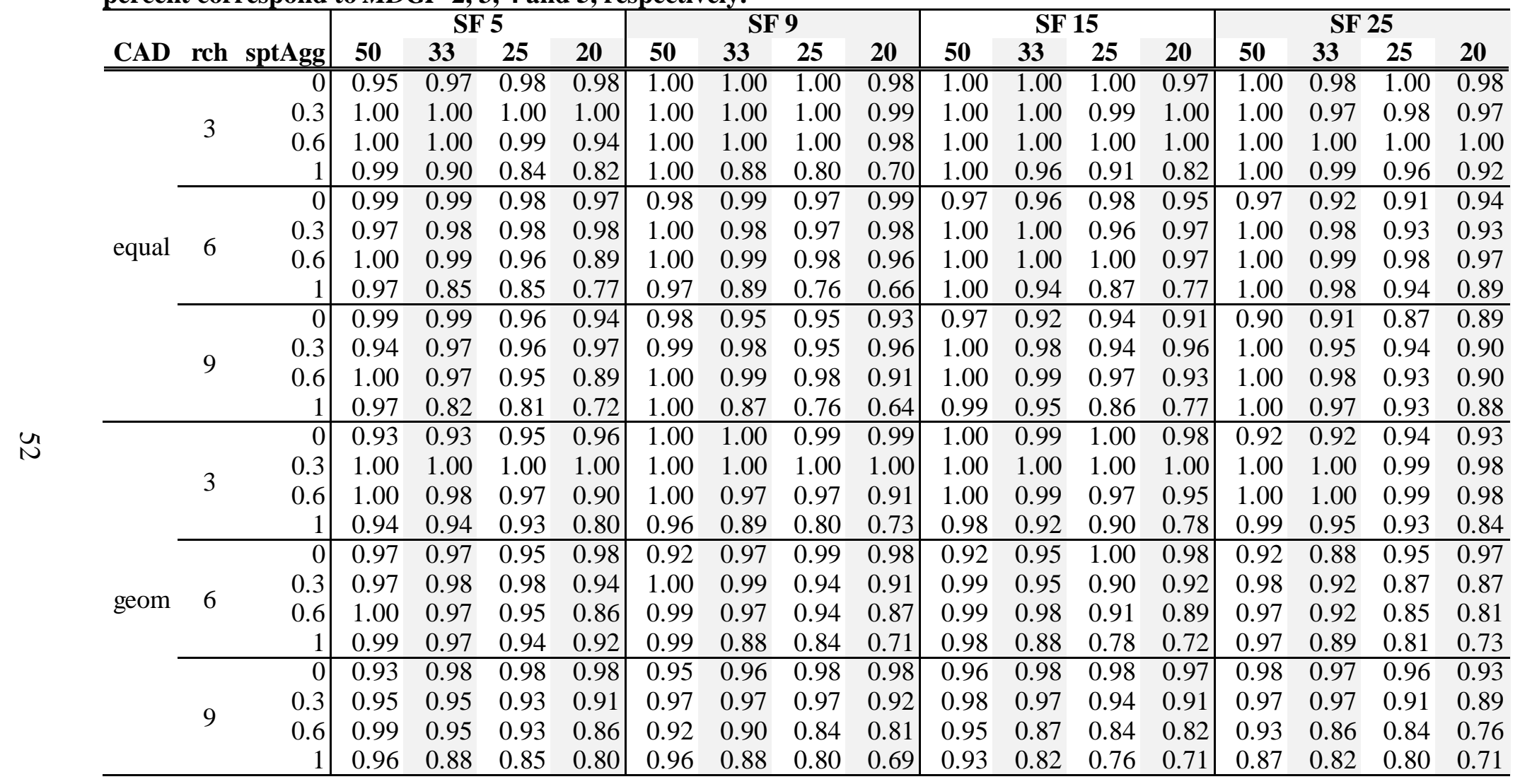


Table 1.8. Differences in class-label fidelity as class-label precision increases (rows within scale factors (SF)) for 24 landscape types varying in class-abundance distribution (CAD), richness ( $\mathrm{rch}$ ) and spatial aggregation (sptAgg). Initial reference for $M D G P$-scaling algorithm of two parts (precision $=\mathbf{5 0 \%}$ ) compared to majority-rule (precision $=100 \%$ ). Class-label precisions of $50,33,25$ and 20 \begin{tabular}{c|c|c|c|c|} 
percent. Colors indicate classified class-label fidelity change as class-label precision increases from 100 to 50, 33, 25 and 20 percent. & SF 15 & SF 25
\end{tabular}

\begin{tabular}{|c|c|c|c|c|c|c|c|c|c|c|c|c|c|c|c|c|c|c|}
\hline \multirow[b]{2}{*}{ CAD } & \multirow[b]{2}{*}{ rch } & \multirow[b]{2}{*}{ sptAgg } & \multicolumn{4}{|c|}{ SF 5} & \multicolumn{4}{|c|}{ SF 9} & \multicolumn{4}{|c|}{ SF 15} & \multicolumn{4}{|c|}{ SF 25} \\
\hline & & & 50 & 33 & 25 & 20 & 50 & 33 & 25 & 20 & 50 & 33 & 25 & 20 & 50 & 33 & 25 & 20 \\
\hline \multirow{12}{*}{ equal } & \multirow{4}{*}{3} & $\begin{array}{ll} & 0\end{array}$ & -0.05 & "0.02 & "0.01 & "0.00 & "0.00 & "0.00 & "0.00 & -0.02 & "0.00 & 0.00 & 0.00 & -0.03 & "0.00 & -0.02 & 0.02 & -0.02 \\
\hline & & 0.3 & 0.00 & 0.00 & 0.00 & 0.00 & 0.00 & 0.00 & 0.00 & 0.00 & 0.00 & 0.00 & -0.01 & 0.01 & 0.00 & -0.03 & 0.01 & -0.02 \\
\hline & & 0.6 & 0.00 & 0.00 & -0.01 & -0.05 & 0.00 & 0.00 & 0.00 & -0.02 & 0.00 & 0.00 & 0.00 & 0.00 & 0.00 & 0.00 & 0.00 & 0.00 \\
\hline & & 1 & -0.02 & -0.09 & -0.06 & -0.01 & 0.00 & -0.12 & -0.09 & -0.09 & 0.00 & -0.04 & -0.05 & -0.09 & 0.00 & -0.01 & -0.03 & -0.04 \\
\hline & \multirow{4}{*}{6} & 0 & -0.01 & 0.00 & -0.01 & -0.01 & -0.02 & 0.01 & -0.03 & 0.03 & -0.03 & -0.01 & 0.02 & -0.03 & -0.03 & -0.05 & -0.01 & 0.03 \\
\hline & & 0.3 & -0.03 & 0.02 & 0.00 & -0.01 & 0.00 & -0.02 & -0.02 & 0.01 & -0.01 & 0.00 & -0.04 & 0.01 & 0.00 & -0.02 & -0.05 & 0.00 \\
\hline & & 0.6 & 0.00 & -0.01 & -0.03 & -0.07 & 0.00 & -0.01 & -0.02 & -0.02 & 0.00 & 0.00 & 0.00 & -0.03 & 0.00 & -0.01 & 0.00 & -0.02 \\
\hline & & 1 & -0.03 & -0.12 & 0.00 & -0.07 & -0.03 & -0.09 & -0.13 & -0.10 & 0.00 & -0.06 & -0.07 & -0.10 & 0.00 & -0.01 & -0.04 & -0.05 \\
\hline & \multirow{4}{*}{9} & 0 & -0.01 & -0.01 & -0.03 & -0.02 & -0.02 & -0.04 & 0.00 & -0.02 & -0.03 & -0.06 & 0.02 & -0.03 & -0.10 & 0.01 & -0.04 & 0.02 \\
\hline & & 0.3 & -0.06 & 0.03 & -0.01 & 0.01 & -0.01 & -0.01 & -0.04 & 0.02 & 0.00 & -0.02 & -0.03 & 0.02 & 0.00 & -0.05 & -0.01 & -0.03 \\
\hline & & 0.6 & 0.00 & -0.03 & -0.03 & -0.05 & 0.00 & -0.01 & -0.01 & -0.06 & 0.00 & -0.01 & -0.01 & -0.04 & 0.00 & -0.02 & -0.05 & -0.03 \\
\hline & & 1 & -0.03 & -0.16 & -0.01 & -0.09 & 0.00 & -0.13 & -0.11 & -0.12 & -0.01 & -0.05 & -0.08 & -0.09 & 0.00 & -0.02 & -0.05 & -0.04 \\
\hline \multirow{12}{*}{ geom } & \multirow{4}{*}{3} & 0 & -0.07 & 0.00 & 0.02 & 0.01 & 0.00 & 0.00 & -0.01 & -0.01 & 0.00 & -0.01 & 0.01 & -0.02 & -0.09 & 0.00 & 0.02 & -0.01 \\
\hline & & 0.3 & 0.00 & 0.00 & 0.00 & 0.00 & 0.00 & 0.00 & 0.00 & 0.00 & 0.00 & 0.00 & 0.00 & -0.01 & 0.00 & 0.00 & -0.01 & 0.00 \\
\hline & & 0.6 & 0.00 & -0.02 & -0.01 & -0.07 & 0.00 & -0.03 & 0.00 & -0.06 & 0.00 & -0.01 & -0.02 & -0.02 & 0.00 & 0.00 & -0.01 & -0.01 \\
\hline & & 1 & -0.06 & -0.01 & -0.01 & -0.13 & -0.04 & -0.07 & -0.09 & -0.06 & -0.02 & -0.07 & -0.02 & -0.12 & -0.01 & -0.04 & -0.02 & -0.09 \\
\hline & \multirow{4}{*}{6} & 0 & -0.03 & 0.00 & -0.02 & 0.03 & -0.08 & 0.05 & 0.02 & -0.01 & -0.08 & 0.03 & 0.05 & -0.02 & -0.03 & -0.03 & 0.06 & 0.03 \\
\hline & & 0.3 & -0.03 & 0.00 & 0.00 & -0.03 & 0.00 & -0.01 & -0.06 & -0.02 & -0.01 & -0.04 & -0.05 & 0.02 & -0.02 & -0.06 & -0.05 & 0.00 \\
\hline & & 0.6 & 0.00 & -0.02 & -0.02 & -0.09 & -0.01 & -0.02 & -0.04 & -0.07 & -0.01 & 0.00 & -0.07 & -0.02 & -0.03 & -0.05 & -0.07 & -0.04 \\
\hline & & 1 & -0.01 & -0.03 & -0.02 & -0.03 & -0.01 & -0.11 & -0.04 & -0.13 & -0.02 & -0.10 & -0.10 & -0.06 & -0.03 & -0.07 & -0.09 & -0.08 \\
\hline & \multirow{4}{*}{9} & 0 & -0.07 & 0.05 & -0.01 & 0.00 & -0.05 & 0.01 & 0.02 & 0.00 & 0.03 & 0.02 & 0.00 & -0.01 & 0.13 & -0.01 & -0.02 & -0.02 \\
\hline & & 0.3 & -0.05 & 0.00 & -0.02 & -0.02 & -0.03 & 0.00 & 0.00 & -0.05 & -0.02 & -0.01 & -0.03 & -0.04 & 0.00 & 0.00 & -0.05 & -0.02 \\
\hline & & 0.6 & -0.01 & -0.04 & -0.01 & -0.08 & -0.08 & -0.02 & -0.06 & -0.03 & -0.05 & -0.07 & -0.03 & -0.02 & -0.03 & -0.07 & -0.03 & -0.07 \\
\hline & & 1 & -0.04 & -0.08 & -0.03 & -0.05 & -0.04 & -0.09 & -0.08 & -0.11 & -0.07 & -0.11 & -0.06 & -0.05 & -0.11 & -0.06 & -0.01 & -0.09 \\
\hline
\end{tabular}

\begin{tabular}{ll|l|l|l|}
\hline Parameter Change $<=-0.1$ & $-0.05-0.09$ & $-0.04-0$ & $>0$ \\
\hline
\end{tabular} 
Table 1.9. Optimization of class-label precision parameter (Optimal Class Prc.) for a scale factor of 15 when considering change in information retention, class-label fidelity and in- to output class-count ratio as class-label precision increases. Landscape types vary in class-abundance distribution (CAD), richness ( $r c h$ ) and spatial aggregation (sptAgg). Optimal Class Prc. suggested on the basis of all three criteria. Colors indicate classified parameter changes as class-label precision increases from 100 to $50,33,25$ and 20 percent.

\begin{tabular}{|c|c|c|c|c|c|c|c|c|c|c|c|c|c|c|c|}
\hline \multirow[b]{2}{*}{ CAD } & \multirow[b]{2}{*}{ rch } & \multirow[b]{2}{*}{ sptAgg } & \multicolumn{4}{|c|}{$\begin{array}{c}\text { Information Retention } \\
\text { Change }\end{array}$} & \multicolumn{4}{|c|}{ Label Fidelity Change } & \multicolumn{4}{|c|}{ Class Ratio Change } & \multirow{2}{*}{$\begin{array}{c}\text { Optimal } \\
\text { Class } \\
\text { Prc. } \\
\end{array}$} \\
\hline & & & $\mathbf{5 0}$ & 33 & 25 & 20 & 50 & 33 & 25 & 20 & 50 & 33 & 25 & 20 & \\
\hline \multirow{12}{*}{ equal } & \multirow{4}{*}{3} & 0 & 23.2 & 5.0 & 2.7 & 2.1 & 0.00 & 0.00 & 0.00 & -0.03 & 0.3 & 1.0 & 0.7 & 0.5 & 25,20 \\
\hline & & 0.3 & 7.8 & 3.0 & 1.5 & 0.9 & 0.00 & 0.00 & -0.01 & 0.01 & 0.7 & 0.7 & 0.8 & 0.7 & 33 \\
\hline & & 0.6 & 3.1 & 1.2 & 0.7 & 0.4 & 0.00 & 0.00 & 0.00 & 0.00 & 0.7 & 0.7 & 0.7 & 0.7 & 50 \\
\hline & & 1 & 1.2 & 0.4 & 0.2 & 0.1 & 0.00 & -0.04 & -0.05 & -0.09 & 0.7 & 0.6 & 0.5 & 0.2 & 100 \\
\hline & \multirow{4}{*}{6} & 0 & 22.2 & 16.0 & 9.3 & 2.2 & -0.03 & -0.01 & 0.02 & -0.03 & 0.7 & 0.1 & -0.2 & 0.9 & 25,20 \\
\hline & & 0.3 & 22.4 & 5.0 & 3.2 & 1.8 & -0.01 & 0.00 & -0.04 & 0.01 & 0.3 & 1.2 & 0.8 & 0.9 & 25 \\
\hline & & 0.6 & 6.1 & 2.3 & 1.2 & 0.7 & 0.00 & 0.00 & 0.00 & -0.03 & 0.8 & 0.8 & 0.8 & 0.7 & 25 \\
\hline & & 1 & 2.7 & 0.8 & 0.4 & 0.1 & 0.00 & -0.06 & -0.07 & -0.10 & 0.8 & 0.6 & 0.4 & 0.2 & 50 \\
\hline & \multirow{4}{*}{9} & $\overline{0}$ & 18.3 & 14.3 & 11.4 & 7.9 & -0.03 & -0.06 & 0.02 & -0.03 & 1.2 & 0.2 & -0.1 & -0.3 & 20 \\
\hline & & 0.3 & 24.9 & 7.7 & 3.3 & 2.1 & 0.00 & -0.02 & -0.03 & 0.02 & 0.1 & 0.9 & 0.7 & 1.1 & 33 \\
\hline & & 0.6 & 10.2 & 3.4 & 1.7 & 0.9 & 0.00 & -0.01 & -0.01 & -0.04 & 0.9 & 0.9 & 0.9 & 0.8 & 50,33 \\
\hline & & 1 & 4.3 & 1.4 & 0.5 & 0.3 & -0.01 & -0.05 & -0.08 & -0.09 & 0.8 & 0.7 & 0.4 & 0.3 & 50 \\
\hline \multirow{12}{*}{ geom } & \multirow{4}{*}{3} & 0 & 18.1 & 4.1 & 2.7 & 1.6 & 0.00 & -0.01 & 0.01 & -0.02 & 0.3 & 0.9 & 0.7 & 0.3 & 33 \\
\hline & & 0.3 & 6.7 & 2.6 & 1.3 & 0.8 & 0.00 & 0.00 & 0.00 & -0.01 & 0.7 & 0.7 & 0.7 & 0.8 & 33 \\
\hline & & 0.6 & 2.4 & 0.9 & 0.5 & 0.3 & 0.00 & -0.01 & -0.02 & -0.02 & 0.7 & 0.6 & 0.6 & 0.6 & 50 \\
\hline & & 1 & 1.0 & 0.3 & 0.1 & 0.0 & -0.02 & -0.07 & -0.02 & -0.12 & 0.6 & 0.4 & 0.3 & 0.2 & 100 \\
\hline & \multirow{4}{*}{6} & 0 & 18.1 & 9.8 & 3.1 & 2.4 & -0.08 & 0.03 & 0.05 & -0.02 & -0.1 & 0.1 & 0.4 & 0.4 & 25,20 \\
\hline & & 0.3 & 11.9 & 3.2 & 1.9 & 1.0 & -0.01 & -0.04 & -0.05 & 0.02 & 0.4 & 0.7 & 0.4 & 0.6 & $\mathbf{5 0}$ \\
\hline & & 0.6 & 4.3 & 1.6 & 0.7 & 0.4 & -0.01 & 0.00 & -0.07 & -0.02 & 0.7 & 0.6 & 0.5 & 0.5 & 50 \\
\hline & & 1 & 1.3 & 0.3 & 0.1 & 0.1 & -0.02 & -0.10 & -0.10 & -0.06 & 0.5 & 0.3 & 0.1 & 0.1 & 100 \\
\hline & \multirow{4}{*}{9} & $\overline{0}$ & 17.7 & 9.6 & 3.2 & 2.7 & 0.03 & 0.02 & 0.00 & -0.01 & -0.4 & 0.1 & 0.4 & 0.3 & 25,20 \\
\hline & & 0.3 & 13.4 & 4.1 & 2.2 & 1.2 & -0.02 & -0.01 & -0.03 & -0.04 & -0.1 & 0.4 & 0.4 & 0.4 & 25,20 \\
\hline & & 0.6 & 4.7 & 1.6 & 0.9 & 0.5 & -0.05 & -0.07 & -0.03 & -0.02 & 0.3 & 0.4 & 0.4 & 0.3 & 33 \\
\hline & & 1 & 2.1 & 0.6 & 0.2 & 0.1 & -0.07 & -0.11 & -0.06 & -0.05 & 0.4 & 0.3 & 0.2 & 0.1 & 50 \\
\hline
\end{tabular}

\begin{tabular}{|c|c|c|c|c|c|c|c|c|c|c|c|}
\hline Parameter Change & $>20$ & $15-20$ & $10-15$ & $5-10$ & $<=-0.1$ & $-0.05--0.09$ & & $<0$ & $0-0.5$ & $0.5-1$ & $>=1$ \\
\cline { 2 - 10 }
\end{tabular} 


\section{FIGURES}

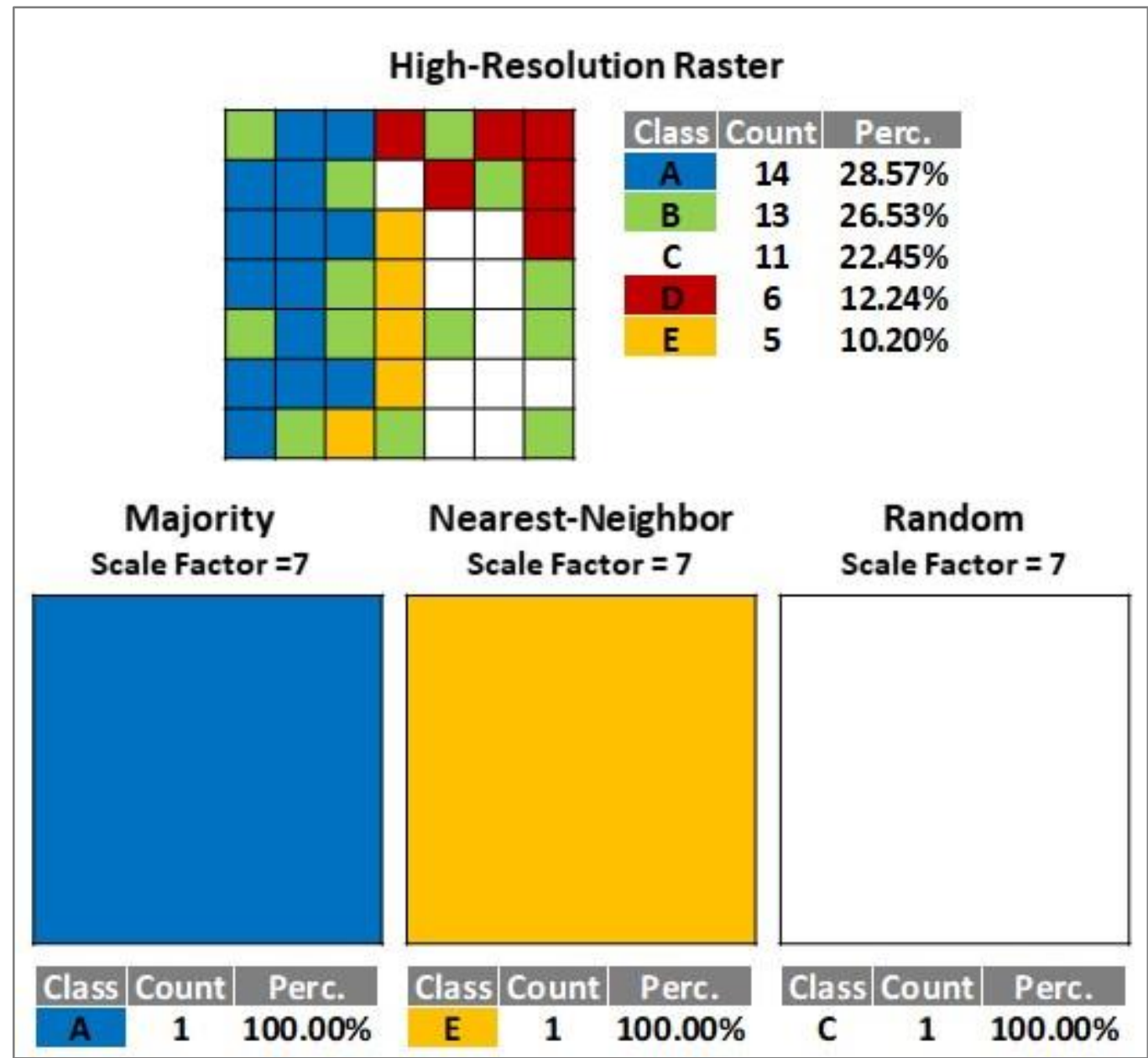

Figure 1.1. Two common issues associated with majority-, nearest-neighbor- and random-rule scaling algorithms. Scaled grid cell (below) represents only one of five original classes, when a mixed class is more representative. In all three cases, four classes are omitted from the scaled class label. The single-class scaled class label over-represents its class with $100 \%$, when in fact that class was present at only $28.57 \%$ for the outcome of the majority rule, $\mathbf{2 2 . 4 5 \%}$ for the random rule and only $\mathbf{1 0 . 2 \%}$ for the nearest-neighbor rule. 

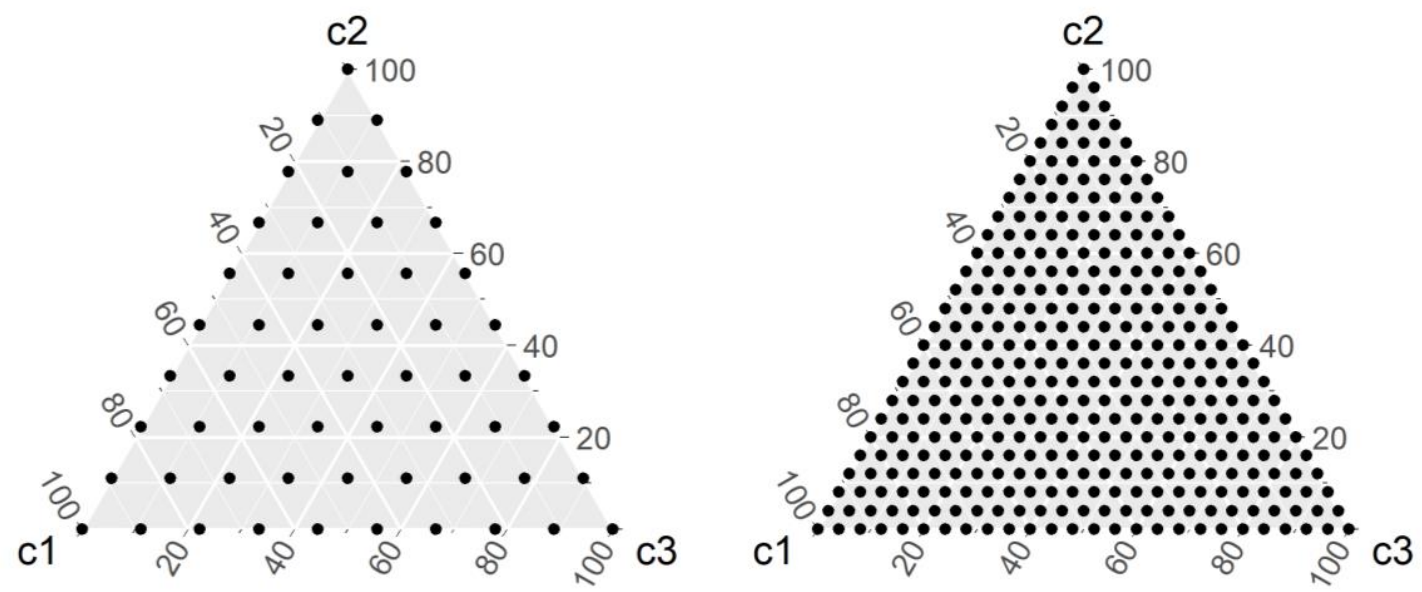

Figure 1.2. Ternary plots for three classes $(\mathrm{c1}-\mathrm{c3})$ and a scale factor of three with 55 possible combinations (left) of the three classes and a scale factor of five with 351 weak combinations (right). Numbers along the axes are proportions of classes present in each combination (dot) in percent. The outer points have one (the apices) or two classes; the inner points are composed of all three classes in differing proportions. 

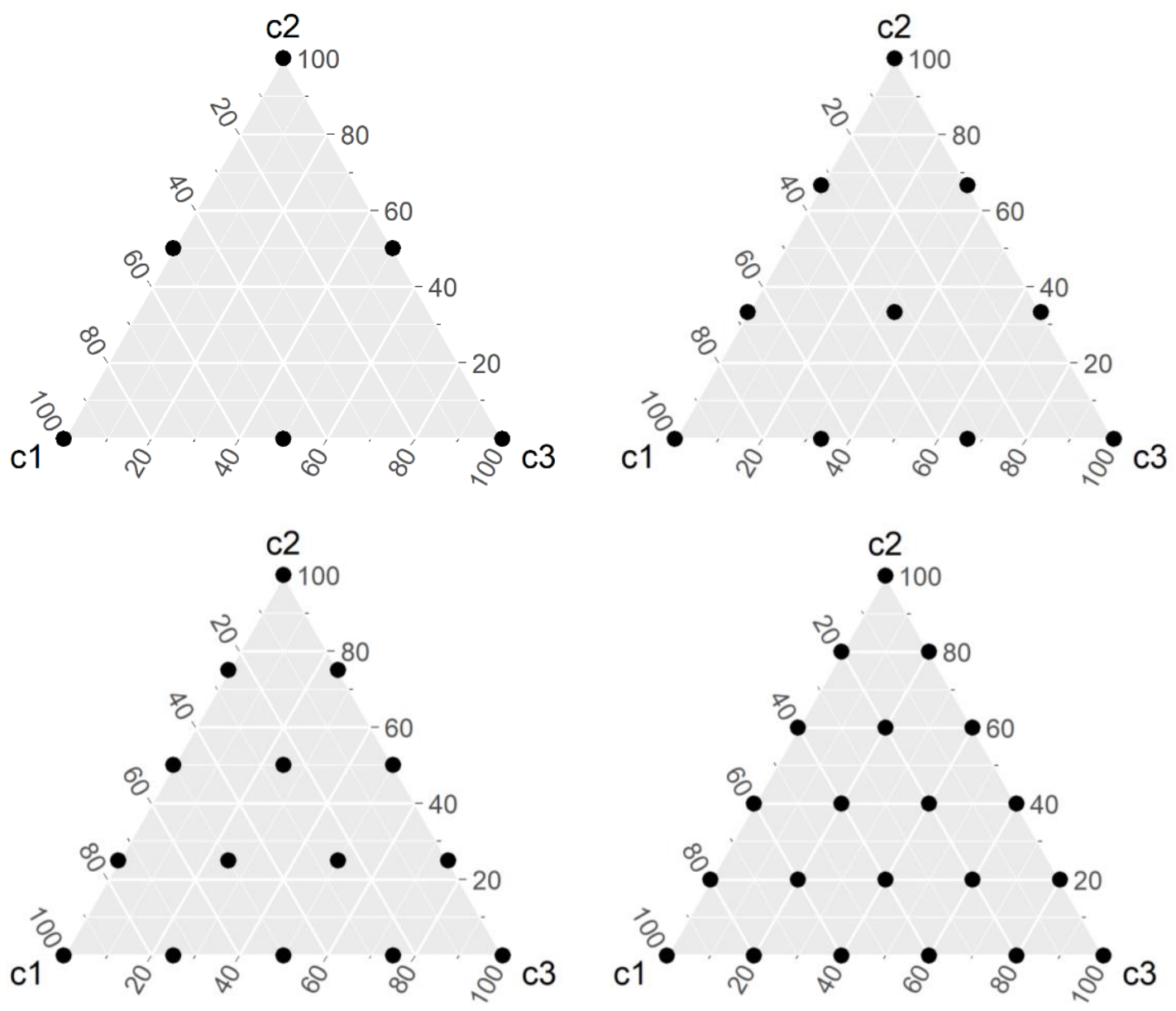

Figure 1.3. Ternary plots of three classes $(\mathrm{c1}-\mathrm{c3})$ for multi-dimensional grid points of $2,3,4$ and 5 parts representing $50 \%, 33.3 \%, 25 \%$ and $20 \%$ class-label precisions (top left to bottom right). Numbers along the axes are proportions of classes present in each combination (dot) in percent. The outer points have one (the apices) or two classes; the inner points, when present, are composed of all three classes in differing proportions. 


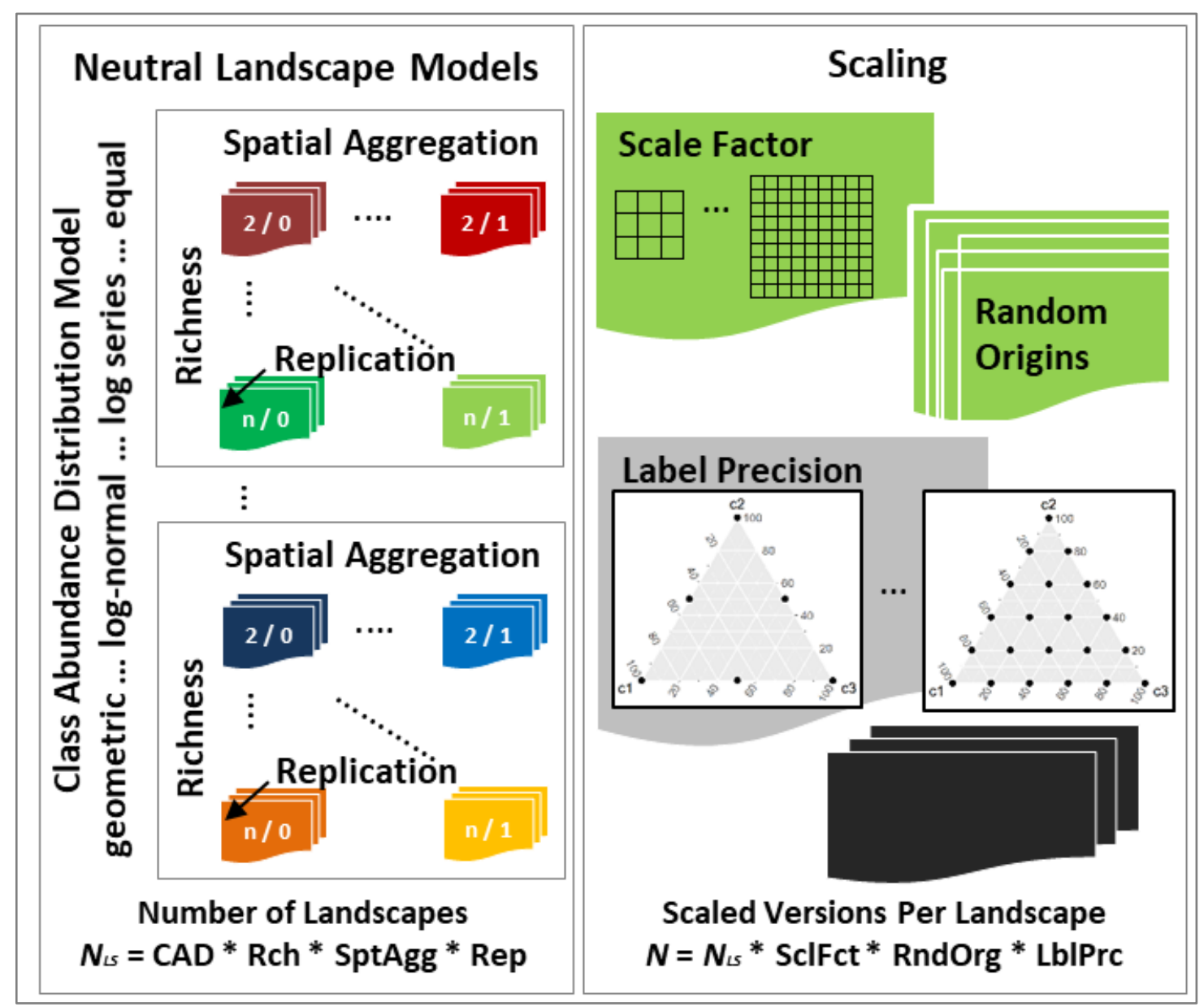

Figure 1.4. Schema of framework to test the effects of landscape characteristics, scale factor and class-label precision on information retention, class-count consistency and class-label fidelity in a full factorial design. 

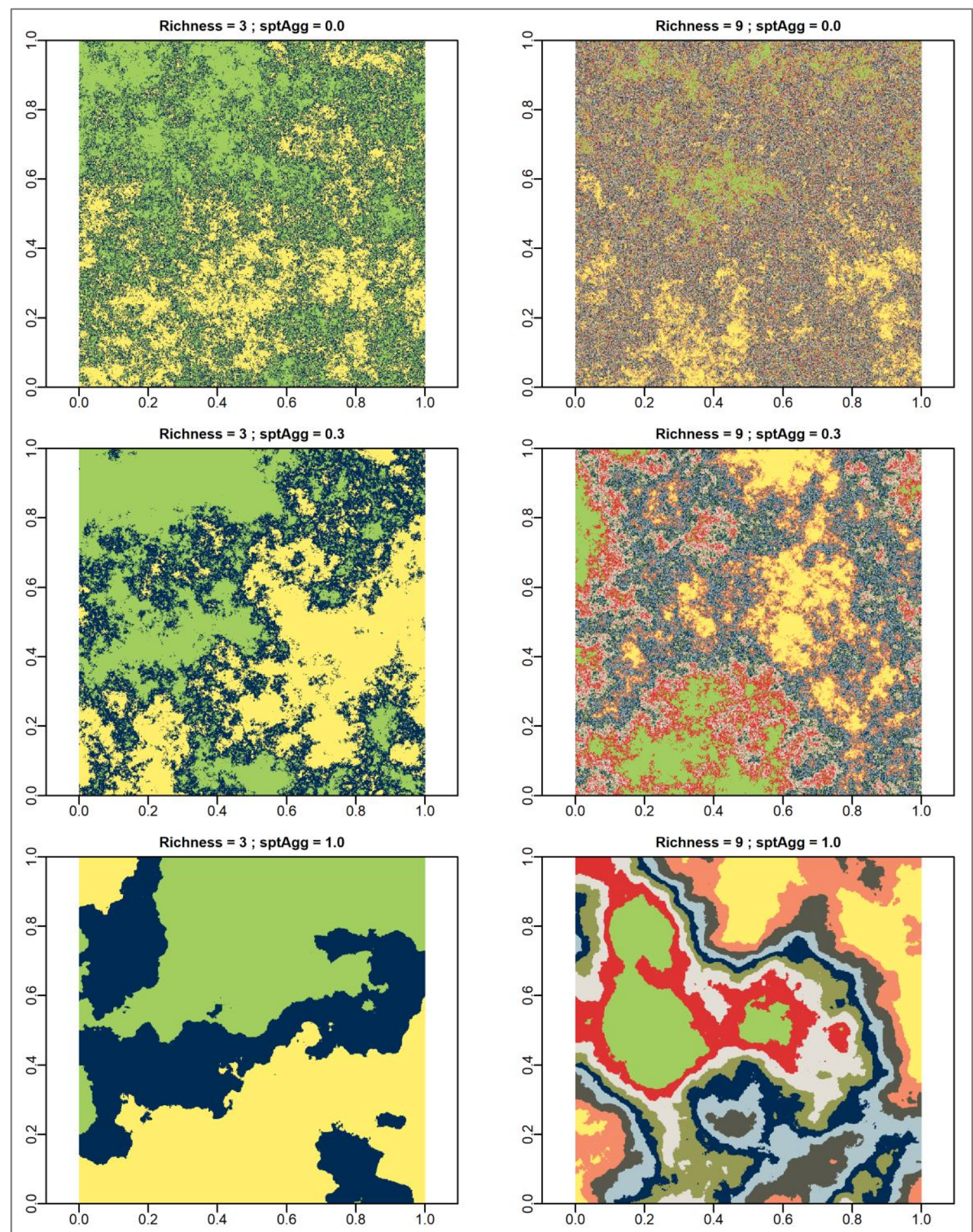

Figure 1.5. Neutral landscapes with equal class-abundance distribution of three classes (left) and nine classes (right) for the lowest spatial aggregation factor (sptAgg) 0.0 (top), an aggregation of 0.3 (middle), and the highest aggregation of 1.0 (bottom). 

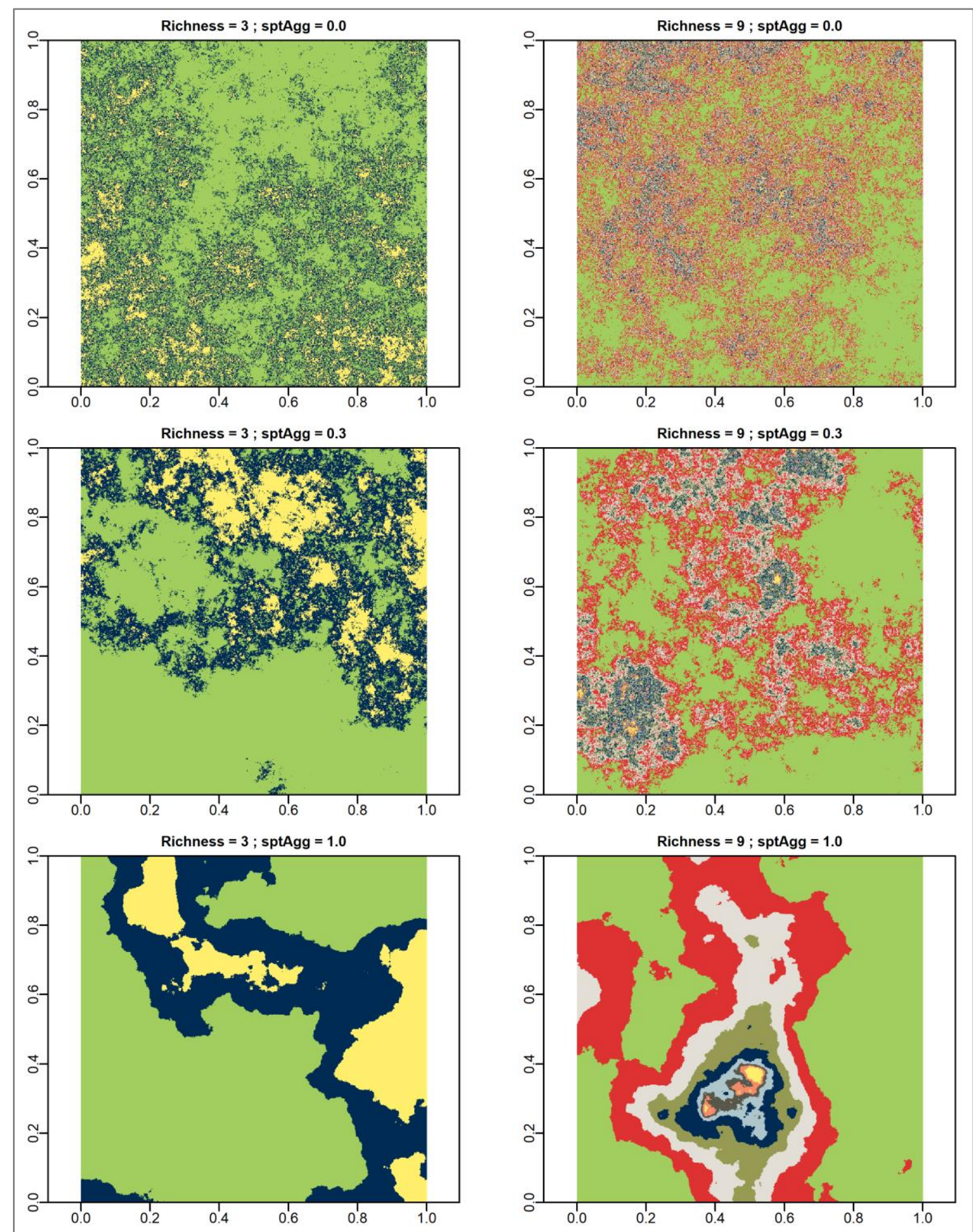

Figure 1.6. Neutral landscapes with geometric class-abundance distribution of three classes (left) and nine classes (right) for the lowest spatial aggregation factor (sptAgg) 0.0 (top), an aggregation of 0.3 (middle), and the highest aggregation of 1.0 (bottom). 


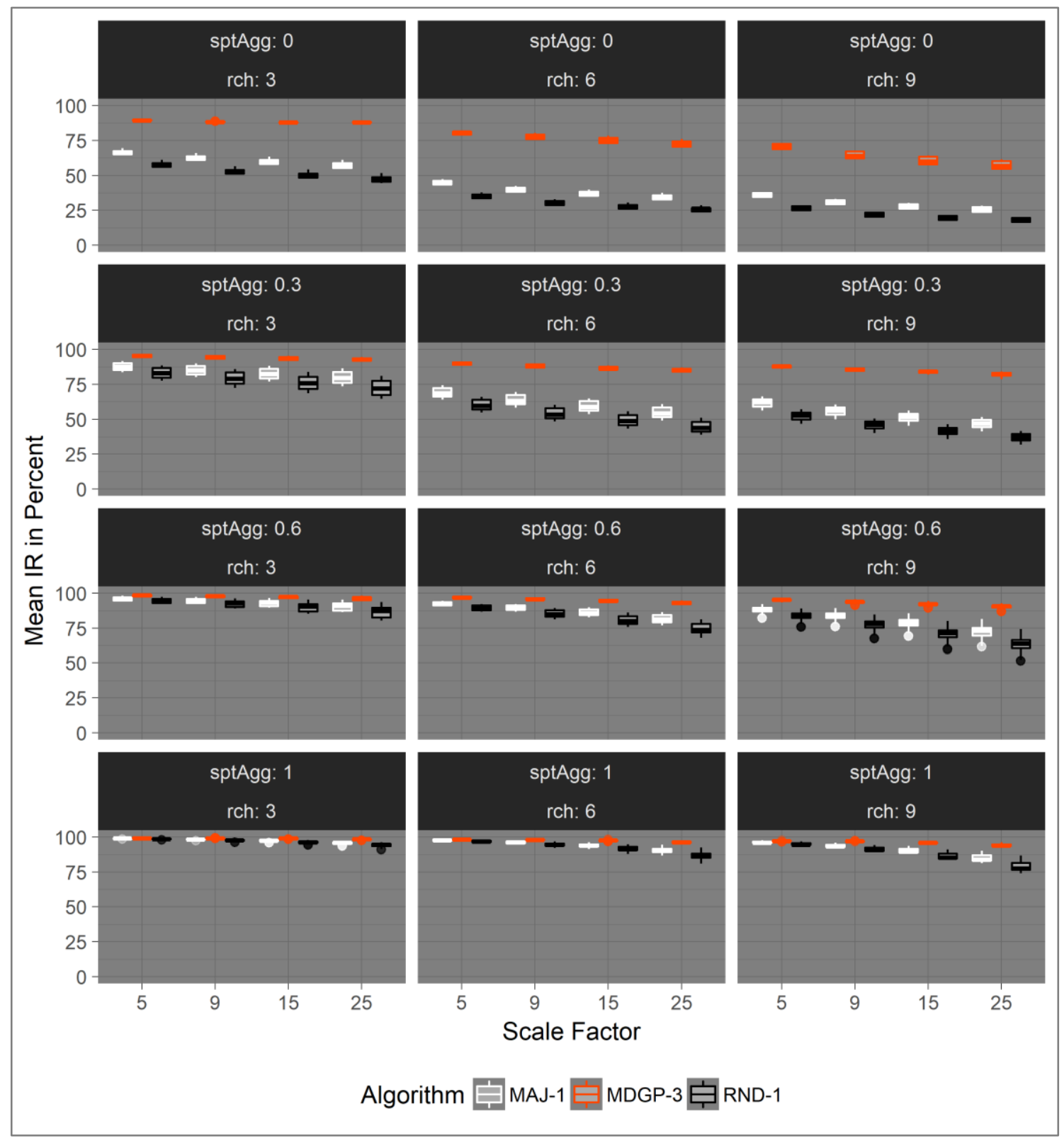

Figure 1.7. Algorithm comparison for mean information retention (IR) with increasing scale factor for three scaling algorithms in simulated landscapes with equal class-abundance distribution. Richness ( $\mathbf{r c h}$ ) varies across columns, while spatial aggregation (sptAgg) varies among rows. $\mathrm{MAJ}-1$ = majority-rule algorithm with $100 \%$ class-label precision, $\mathrm{MDGP}-3=$ multi-dimensional grid point algorithm with class-label precision of 33\%, RND-1 = randomrule algorithm with $100 \%$ class-label precision. 


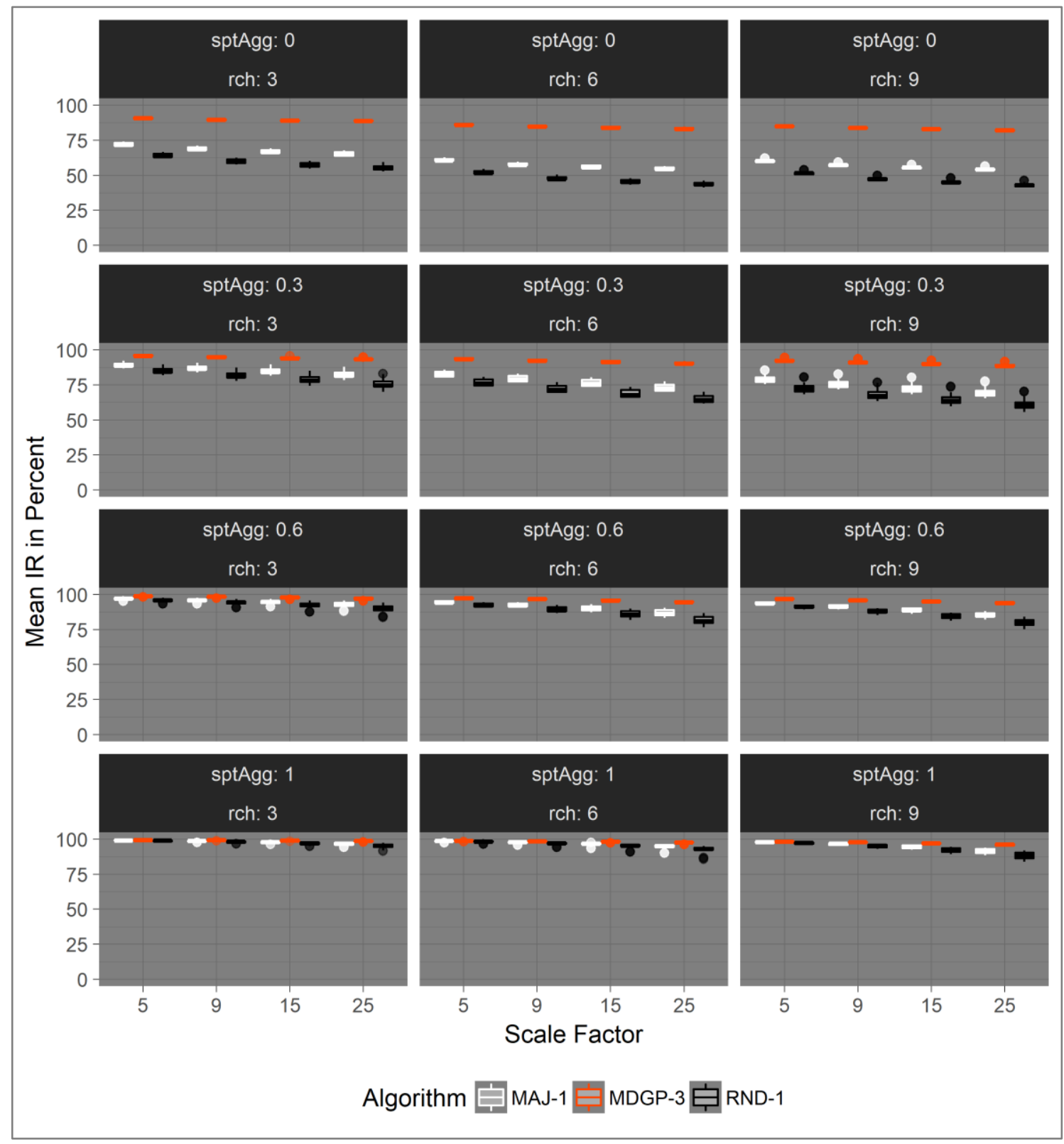

Figure 1.8. Algorithm comparison of mean information retention (IR) with increasing scale factor for three scaling algorithms in simulated landscapes with geometric class-abundance distribution. Richness (rch) increases across columns, while spatial aggregation (sptAgg) increases down rows. MAJ-1 = majority-rule algorithm with $100 \%$ class-label precision, MDGP-3 = multi-dimensional grid point algorithm with class-label precision of 33\%, RND-1 $=$ random-rule algorithm with $100 \%$ class-label precision. 


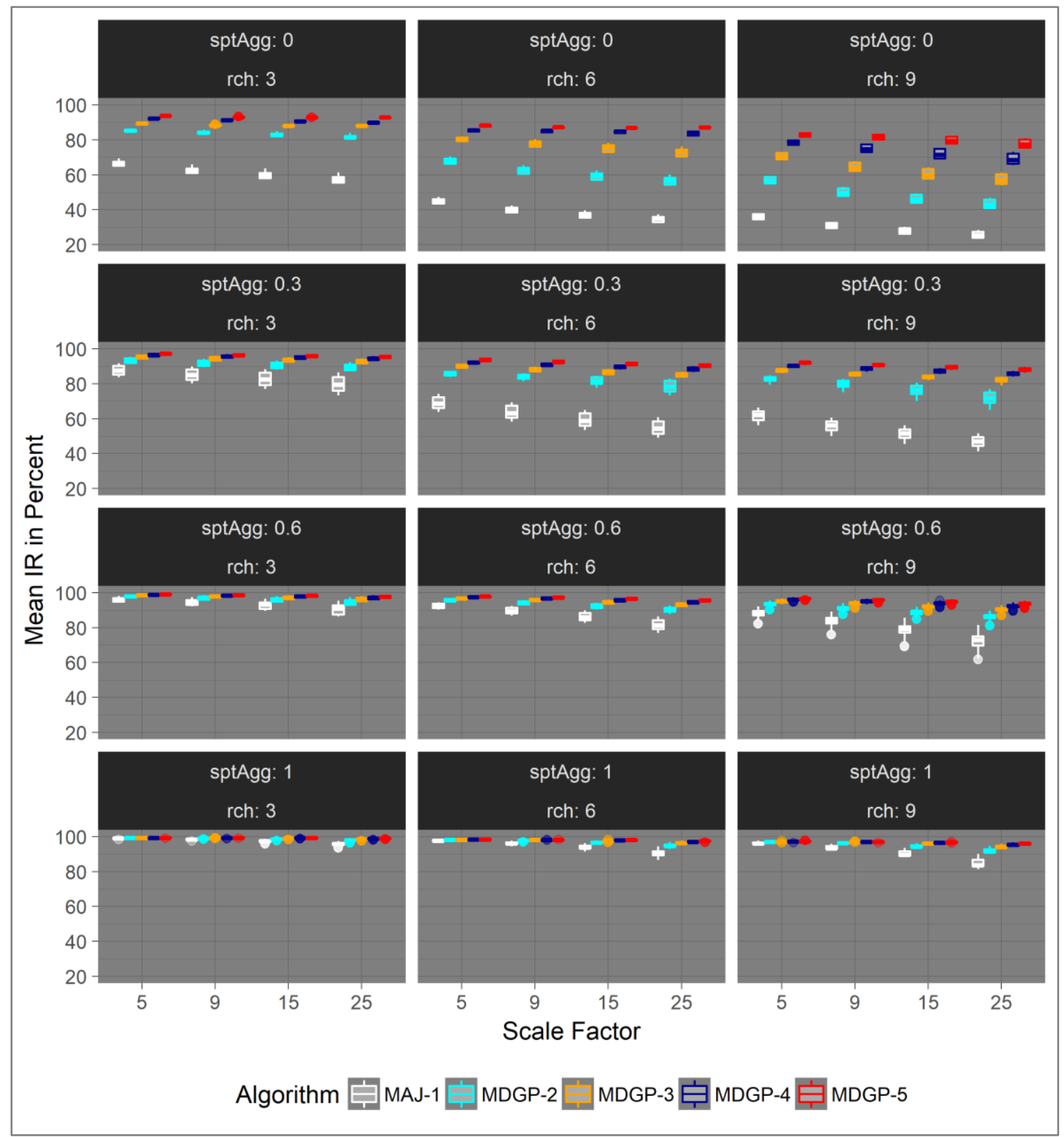

Figure 1.9. Sensitivity of information retention (IR) to landscape characteristics and scaling parameters for landscapes with equal class-abundance distribution. Richness (rch) increases across columns, while spatial aggregation (sptAgg) increases down rows. MAJ-1 = majorityrule algorithm with $100 \%$ class-label precision, MDGP = multi-dimensional grid point algorithm with $2=50 \%, 3=33 \%, 4=25 \%$ and $5=20 \%$ class-label precision. 


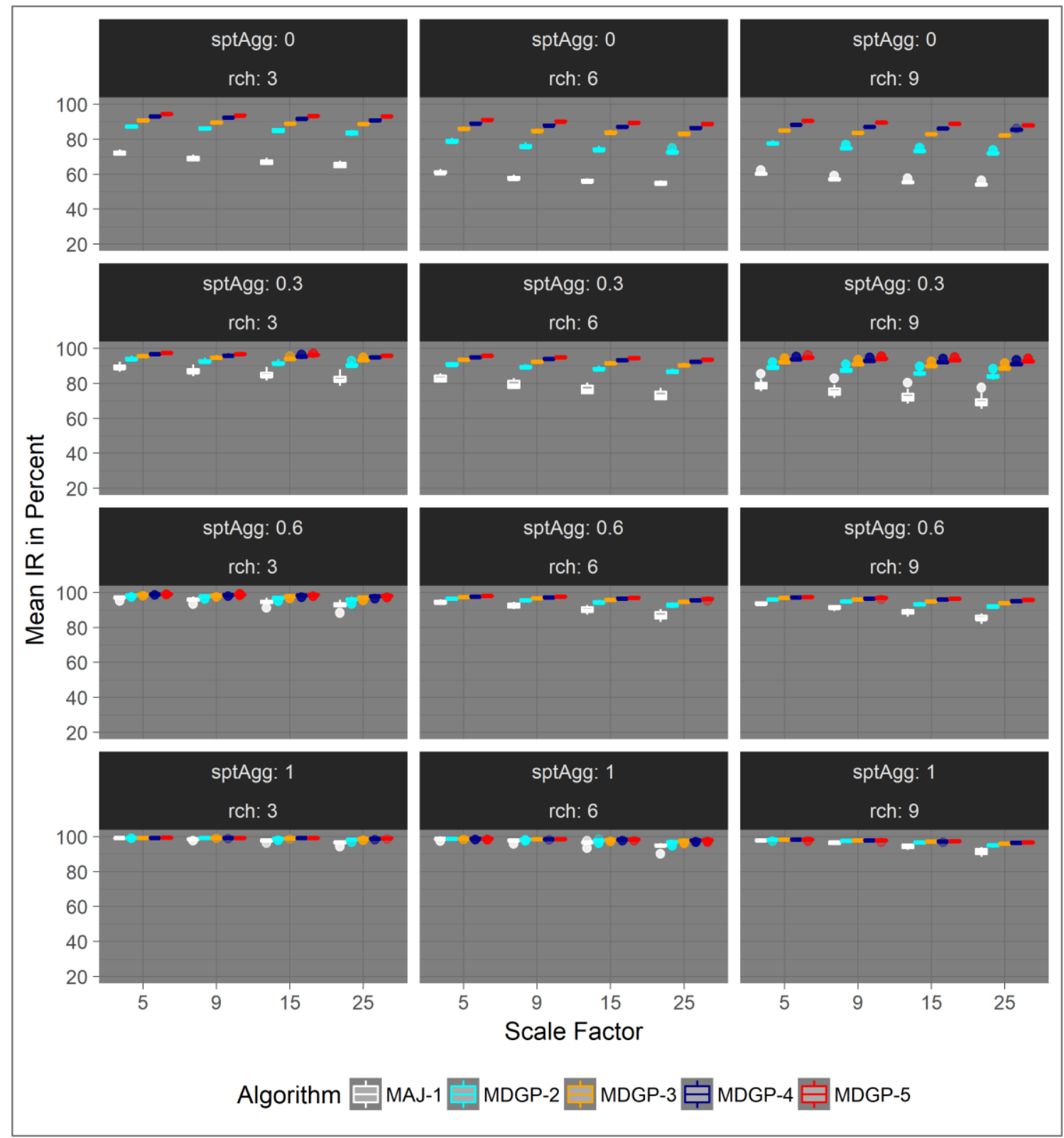

Figure 1.10. Sensitivity of information retention (IR) to landscape characteristics and scaling parameters for landscapes with geometric class-abundance distribution Richness (rch) increases across columns, while spatial aggregation (sptAgg) increases down rows. MAJ-1 = majority-rule algorithm with $100 \%$ class-label precision, MDGP $=$ multi-dimensional grid point algorithm with $2=50 \%, 3=33 \%, 4=25 \%$ and $5=20 \%$ class-label precision. 


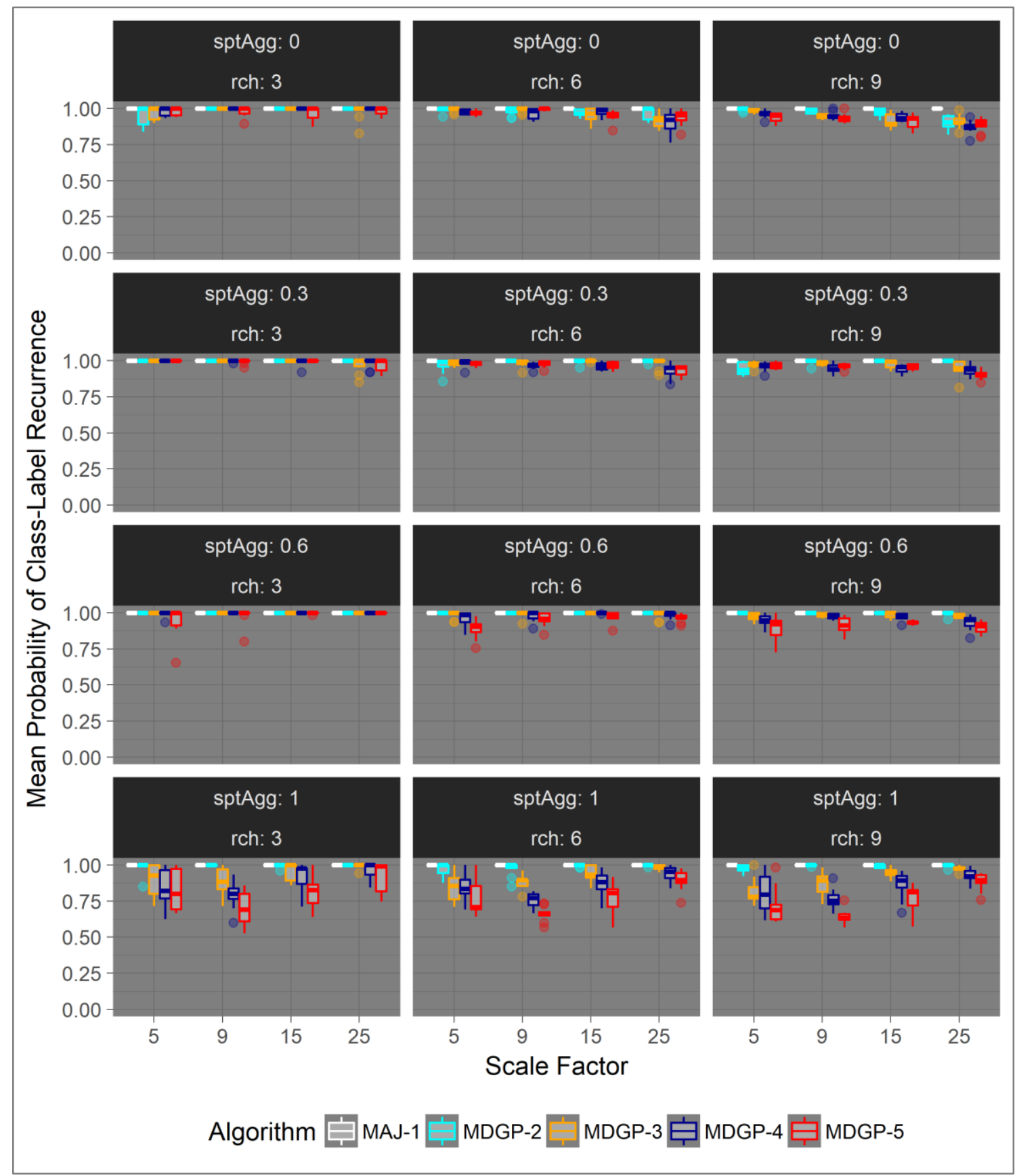

Figure 1.11. Sensitivity of class-label fidelity evaluated across all landscape iterations with the same characteristics for five random origins $(N=50)$ for landscapes with equal classabundance distribution. Richness (rch) increases across columns, while spatial aggregation (sptAgg) increases down rows. MAJ-1 = majority-rule algorithm with $100 \%$ class-label precision, MDGP = multi-dimensional grid point algorithm with $2=50 \%, 3=33 \%, 4=25 \%$ and $5=20 \%$ class-label precision. 


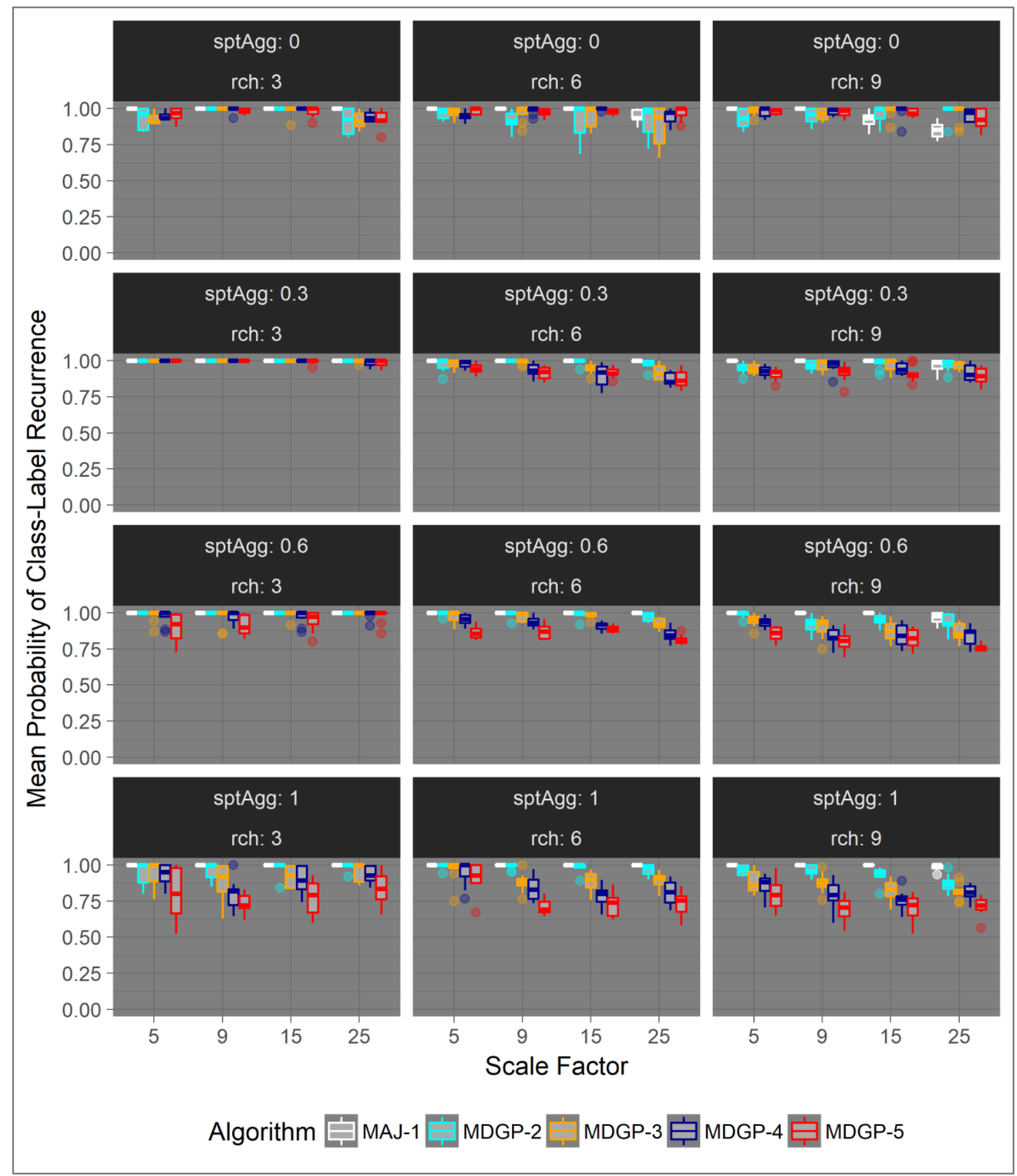

Figure 1.12. Sensitivity of class-label fidelity evaluated across all landscape iterations with the same characteristics for five random origins $(N=50)$ for landscapes with geometric classabundance distribution. Richness (rch) increases across columns, while spatial aggregation (sptAgg) increases down rows. MAJ-1 = majority-rule algorithm with $100 \%$ class-label precision, MDGP = multi-dimensional grid point algorithm with $2=50 \%, 3=33 \%, 4=25 \%$ and $5=20 \%$ class-label precision. 


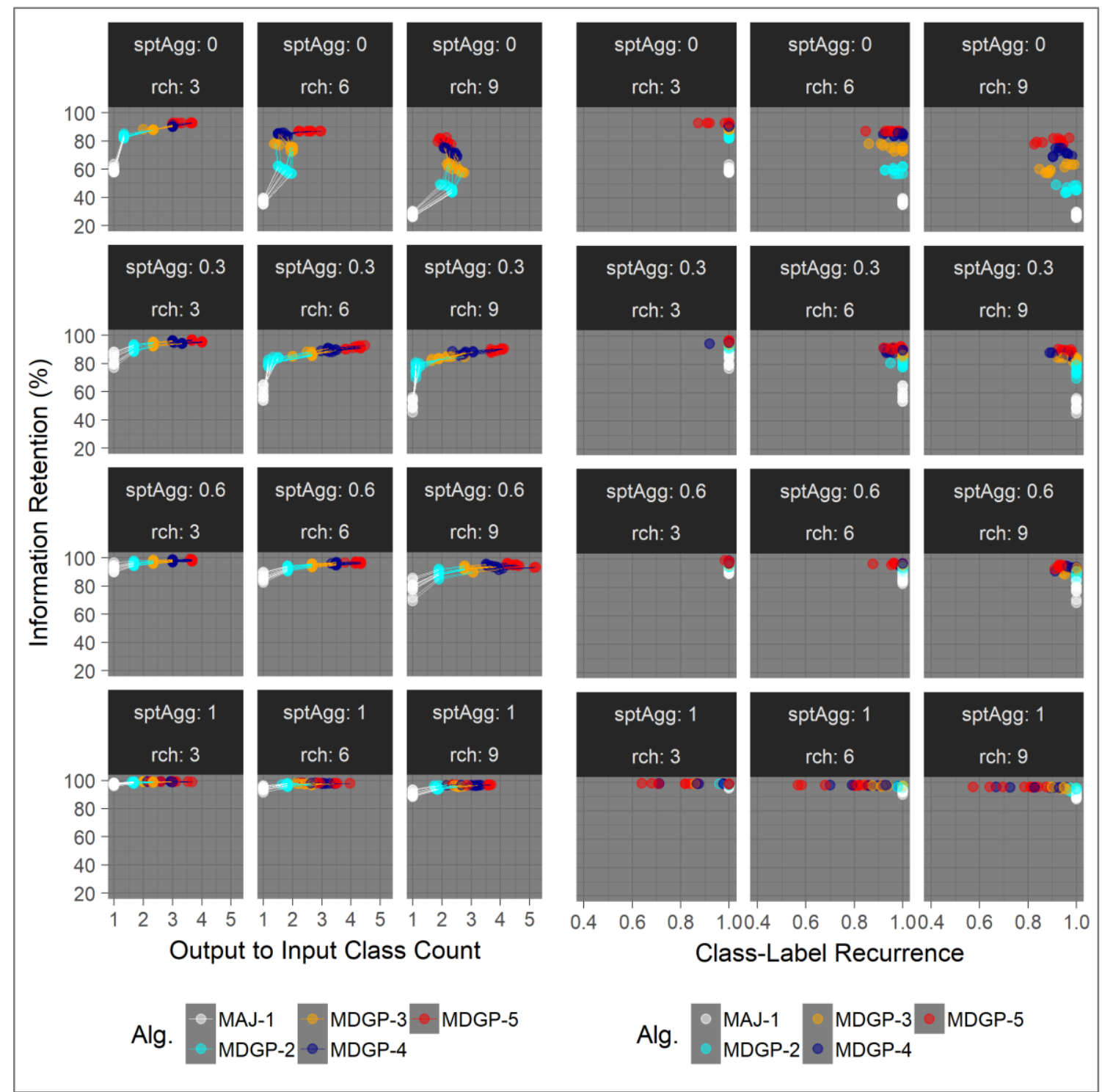

Figure 1.13. Relationships of information retention to class-count ratio (Output to Input Class Count, on left) and class-label fidelity (Class-Label Recurrence, on right) for landscapes with equal class-abundance distribution and a scale factor of $15(N=225$ original grid cells). Within each variable, richness ( $\mathrm{rch}$ ) increases across columns, while spatial aggregation (sptAgg) increases down rows. MAJ-1 = majority-rule algorithm with $100 \%$ class-label precision, MDGP = multi-dimensional grid point algorithm with $2=50 \%, 3=$ $33 \%, 4=25 \%$ and $5=20 \%$ class-label precision. Alg. $=$ Algorithm. 


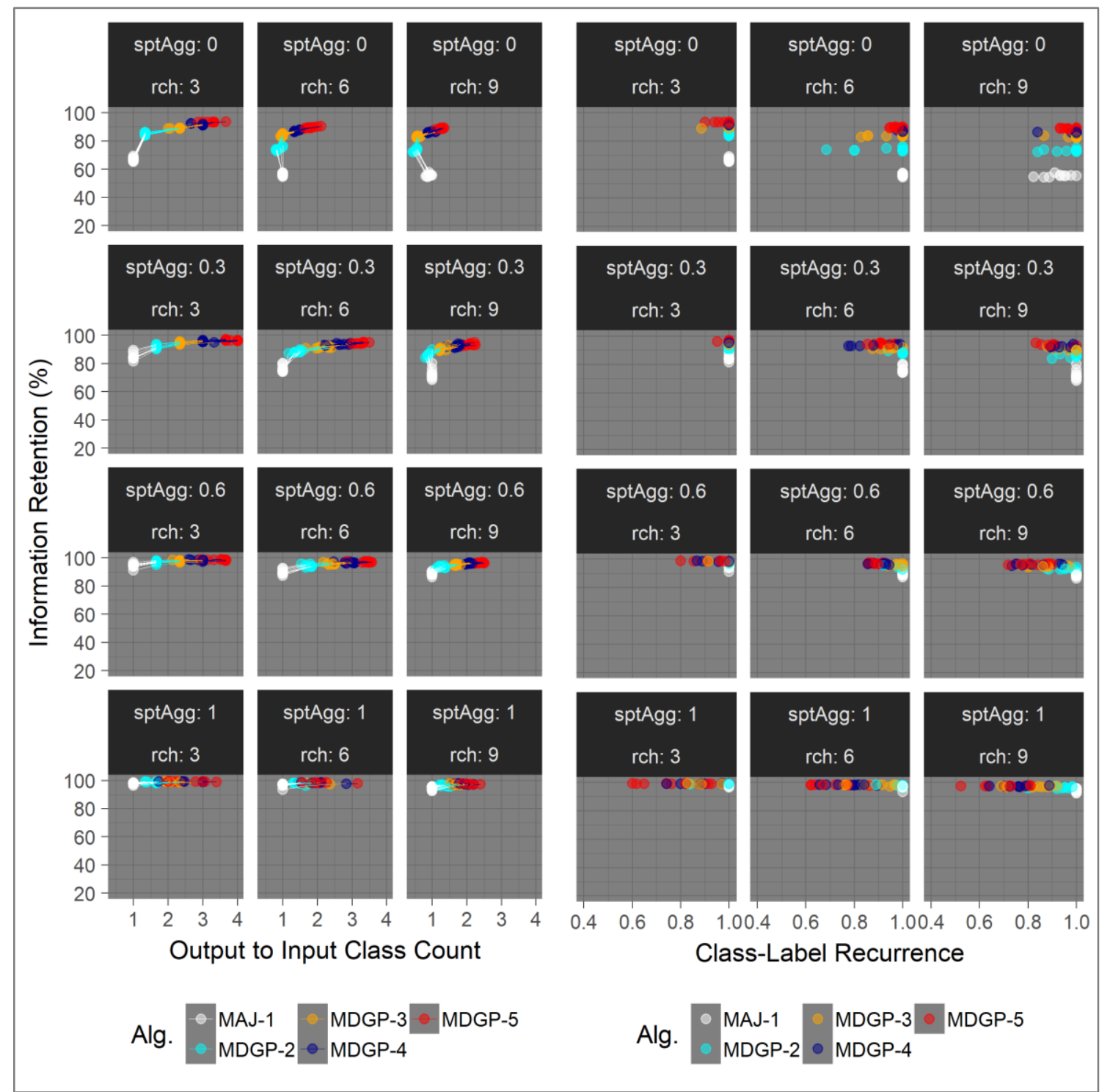

Figure 1.14. Relationships of information retention to class-count ratio (Output to Input Class Count, on left) and class-label fidelity (Class-Label Recurrence, on right) for landscapes with geometric class-abundance distribution and a scale factor of $15(N=225$ original grid cells). Within each variable, richness ( $(\mathrm{rch})$ increases across columns, while spatial aggregation (sptAgg) increases down rows. MAJ-1 = majority-rule algorithm with $100 \%$ class-label precision, MDGP = multi-dimensional grid point algorithm with $2=50 \%, 3$ $=33 \%, 4=25 \%$ and $5=20 \%$ class-label precision. Alg. = Algorithm. 


\section{REFERENCES}

Aitchison, J. 1986. The Statistical Annalysis of Compositional Data. Monographs on Statistics and Applied Probability. London: Chapman and Hall. https://doi.org/10.1007/978-94-009-4109-0.

Aitchison, J., C. Barceló-Vidal, J. A. Martín-Fernández, and V. Pawlowsky-Glahn. 2000. "Logratio Analysis and Compositional Distance." Mathematical Geology 32 (3):271-75. https://doi.org/10.1023/A:1007529726302.

Aitchison, J., and J. J. Egozcue. 2005. "Compositional Data Analysis: Where Are We and Where Should We Be Heading?” Mathematical Geology 37 (7):829-50. https://doi.org/10.1007/s11004-005-7383-7.

Alonso, D., and A. J. McKane. 2004. "Sampling Hubbell's Neutral Theory of Biodiversity." Ecology Letters 7 (10):901-10. https://doi.org/10.1111/j.14610248.2004.00640.x.

Arbelaitz, O., I. Gurrutxaga, J. Muguerza, J. Pérez, and I. Perona. 2013. “An Extensive Comparative Study of Cluster Validity Indices." Pattern Recognition 46 (1):24356. https://doi.org/10.1016/J.PATCOG.2012.07.021.

Bivand, R., T. Keitt, and B. Rowlingson. 2013. "Rgdal: Bindings for the Geospatial Data Abstraction Library". R package version 1.3. http://cran.rproject.org/package=rgdal.

Boutin, C., and P. A. Keddy. 1993. "A Functional Classification of Wetland Plants." Journal of Vegetation Science 4 (5):591-600. https://doi.org/10.2307/3236124.

Braun-Blanquet, J. 1932. Plant Sociology-The Study of Plant Communities. 1st ed. London, UK: McGraw-Hill Book Company.

1964. Pflanzensoziologie, Grundzüge Der Vegetationskunde. 3. Auflage. Wien: Springer Verlag. http://dx.doi.org/10.1007/978-3-7091-8110-2.

De Cáceres, M., X. Font, P. Vicente, and F. Oliva. 2009. "Numerical Reproduction of Traditional Classifications and Automatic Vegetation Identification." Journal of Vegetation Science 20 (4):620-28. https://doi.org/10.1111/j.16541103.2009.01081.x.

De Cáceres, M., and S. K. Wiser. 2012. "Towards Consistency in Vegetation Classification." Journal of Vegetation Science 23 (2):387-93. https://doi.org/10.1111/j.1654-1103.2011.01354.x. 
Cingolani, A. M., M. Cabido, D. E. Gurvich, D. Renison, S. Díaz. 2007. "Filtering Processes in the Assembly of Plant Communities: Are Species Presence and Abundance Driven by the Same Traits?" Journal of Vegetation Science 18 (6):911-20. https://doi.org/10.1111/j.1654-1103.2007.tb02607.x.

Clements, F. E. 1916. Plant Succession; an Analysis of the Development of Vegetation. Washington D.C.: Carnegie Institution of Washington.

Coulston, J. W., N. Zaccarelli, K. H. Riitters, F. H. Koch, and G. Zurlini. 2014. "The Spatial Scan Statistic: A New Method for Spatial Aggregation of Categorical Raster Maps." Quantitative Methods. ArXiv E-Prints August, 1-14. http://arxiv.org/abs/1408.0164.

Egozcue, J. J., V. Pawlowsky-Glahn, G. Mateu-Figueras, and C. Barceló-Vidal. 2003. "Isometric Logratio Transformations for Compositional Data Analysis." Mathematical Geology 35 (3):279-300. https://doi.org/10.1023/A:1023818214614.

Etherington, T. R., E. P. Holland, and D. O'Sullivan. 2015. "NLMpy: A Python Software Package for the Creation of Neutral Landscape Models within a General Numerical Framework." Edited by T. Poisot. Methods in Ecology and Evolution 6 (2):164-68. https://doi.org/10.1111/2041-210X.12308.

Fahrig, L. 1991. "Simulating Methods for Developing General Landscape-Level Hypotheses of Single-Species Dynamics." In Quantitative Methods in Landscape Ecology: The Analysis and Interpretation of Landscape Heterogeneity, edited by M. G. Turner R. H. Gardner, 82:417-42. New York: Springer-Verlag.

Fournier, A., D. Fussell, and L. Carpenter. 1982. "Computer Rendering of Stochastic Models." Communications of the ACM 25 (6):371-84. https://doi.org/10.1145/358523.358553.

Francis, J. M., and J. M. Klopatek. 2000. "Multiscale Effects of Grain Size on Landscape Pattern Analysis.” Annals of GIS 6 (1):27-37.

Gann, D., J. H. Richards, and H. Biswas. 2012. "Determine the Effectiveness of Vegetation Classification Using WorldView 2 Satellite Data for the Greater Everglades.” West Palm Beach, FL: South Florida Water Management District. http://digitalcommons.fiu.edu/gis/25.

Gardner, R. H. 1991. "Pattern, Process, and Predictability: The Use of Neutral Models for Landscape Analysis." In Quantitative Methods in Landscape Ecology: The Analysis and Interpretation of Landscape Heterogeneity, edited by M. G. Turner R. H. Gardner, 82:289-307. New York: Springer-Verlag. 
Gleason, H. A. 1939. "The Individualistic Concept of the Plant Association." American Midland Naturalist 21:92-110.

Gotelli, N. J., and G. R. Graves. 1996. Null Models in Ecology. Washington, D.C.: Smithsonian Institution Press.

He, H. S., S. J. Ventura, and D. J. Mladenoff. 2002. "Effects of Spatial Aggregation Approaches on Classified Satellite Imagery." International Journal of Geographical Information Science 16 (1):93-109. https://doi.org/10.1080/13658810110075978.

Hijmans, R. J., and J. van Etten. 2010. "Raster: Geographic Analysis and Modeling with Raster Data". R Package Version 2.6. https://cran.rproject.org/web/packages/raster/index.html.

Hubbell, S. P. 2005. "Neutral Theory in Community Ecology and the Hypothesis of Functional Equivalence." Functional Ecology 19 (1):166-72. https://doi.org/10.1111/j.0269-8463.2005.00965.x.

— 2006. "Neutral Theory and the Evolution of Ecological Equivalence." Ecology 87 (6):1387-98. https://doi.org/10.1890/0012-9658(2006)87[1387:ntateo]2.0.co;2.

Johnson, G. D., W. L. Myers, G. P. Patil, and C. Taillie. 2001. "Fragmentation Profiles for Real and Simulated Landscapes." Environmental and Ecological Statistics 8 (1):520.

Jonsson, B. G., and J. Moen. 1998. "Patterns in Species Associations in Plant Communities: The Importance of Scale." Journal of Vegetation Science 9 (3):32732. https://doi.org/10.2307/3237097.

Ju, J., S. Gopal, and E. D. Kolaczyk. 2005. "On the Choice of Spatial and Categorical Scale in Remote Sensing Land Cover Classification." Remote Sensing of Environment 96 (1):62-77. https://doi.org/10.1016/j.rse.2005.01.016.

Keddy, P. A. 1992. "Assembly and Response Rules: Two Goals for Predictive Community Ecology.” Journal of Vegetation Science 3 (2):157-64. https://doi.org/10.2307/3235676.

Keddy, P. A. 1993. "Do Ecological Communities Exist? A Reply to Bastow Wilson." Journal of Vegetation Science 4 (1):135-36. https://doi.org/10.2307/3235741.

Lam, N. S.-N., and D. A. Quattrochi. 1992. "On the Issues of Scale, Resolution, and Fractal Analysis in the Mapping Sciences." The Professional Geographer 44 (1):88-98. https://doi.org/10.1111/j.0033-0124.1992.00088.x. 
Martín-Fernández, J. A., C. Barceló-Vidal, and V. Pawlowsky-Glahn. 2003. "Dealing with Zeros and Missing Values in Compositional Data Sets Using Nonparametric Imputation." Mathematical Geology 35 (3):253-78. https://doi.org/10.1023/A:1023866030544.

Martín-Fernández, J. A., K. Hron, M. Templ, P. Filzmoser, and J. Palarea-Albaladejo. 2014. "Bayesian-Multiplicative Treatment of Count Zeros in Compositional Data Sets." Statistical Modelling 15 (2):134-58. https://doi.org/10.1177/1471082X14535524.

Mas, J.-F., Y. Gao, and J. A. Navarrete Pacheco. 2010. "Sensitivity of Landscape Pattern Metrics to Classification Approaches." Forest Ecology and Management 259 (7):1215-24. https://doi.org/10.1016/j.foreco.2009.12.016.

McGill, B. J., R. S. Etienne, J. S. Gray, D. Alonso, M. J. Anderson, H. K. Benecha, M. Dornelas, et al. 2007. "Species Abundance Distributions: Moving beyond Single Prediction Theories to Integration within an Ecological Framework." Ecology Letters 10 (10):995-1015. https://doi.org/10.1111/j.1461-0248.2007.01094.x.

Milligan, G. W., and M. C. Cooper. 1985. “An Examination of Procedures for Determining the Number of Clusters in a Data Set." Psychometrika 50 (2):159-79. https://doi.org/10.1007/BF02294245.

Motomura, I. 1932. "On the Statistical Treatment of Communities.” Zoological Magazine 44:379-383.

Mucina, L., J. H. Schaminée, and J. S. Rodwell. 2000. “Common Data Standards for Recording Relevés in Field Survey for Vegetation Classification.” Journal of Vegetation Science 11 (5):769-72. https://doi.org/10.2307/3236581.

O’Neill, R. V., C. T. Hunsaker, S. P. Timmins, B. L. Jackson, K. B. Jones, K. H. Riitters, and J. D. Wickham. 1996. "Scale Problems in Reporting Landscape Pattern at the Regional Scale.” Landscape Ecology 11 (3):169-80. https://doi.org/10.1007/BF02447515.

O’Neill, R. V., J. R. Krummel, R. H. Gardner, G. Sugihara, B. Jackson, D. L. DeAngelis, B. T. Milne, et al. 1988. "Indices of Landscape Pattern." Landscape Ecology 1 (3):153-62.

Ostapowicz, K., P. Vogt, K. H. Riitters, J. Kozak, and C. Estreguil. 2008. "Impact of Scale on Morphological Spatial Pattern of Forest.” Landscape Ecology 23 (9):1107-17. https://doi.org/10.1007/s10980-008-9271-2. 
Palmer, M. W. 1992. "The Coexistence of Species in Fractal Landscapes." The American Naturalist 139 (2):375-97.

Parker, V. T. 2001. "Conceptual Problems and Scale Limitations of Defining Ecological Communities: A Critique of the CI Concept (Community of Individuals)." Perspectives in Plant Ecology, Evolution and Systematics 4 (2):80-96.

Quattrochi, D. A. 1991. "Remote Sensing for Analysis of Landscapes: An Introduction." In Quantitative Methods in Landscape Ecology: The Analysis and Interpretation of Landscape Heterogeneity, edited by M. G. Turner R. H. Gardner, 82:51-76. New York: Springer-Verlag.

R Core Team. 2013. "R: A Language and Environment for Statistical Computing". R Foundation for Statistical Computing. Vienna, Austria.

Revolution Analytics, and Steve Weston. 2013. "Foreach: Foreach Looping Construct for R.” R package Version 1.4.3. http://cran.r-project.org/package=foreach.

Riitters, K. H., R. V. O'Neill, C. T. Hunsaker, J. D. Wickham, D. H. Yankee, S. P. Timmins, K. B. Jones, and B. L. Jackson. 1995. "A Factor Analysis of Landscape Pattern and Structure Metrics.” Landscape Ecology 10 (1):23-39. https://doi.org/10.1007/BF00158551.

Robertson, P. A. 1980. "Comparisons among Three Hierarchical Classification Techniques Using Simulated Coenoplanes.” Plant Ecology 40 (3):175-83. https://doi.org/10.1007/bf00228483.

Rosindell, J., S. P. Hubbell, and R. S. Etienne. 2011. "The Unified Neutral Theory of Biodiversity and Biogeography at Age Ten." Trends in Ecology \& Evolution 26 (7):340-48. https://doi.org/10.1016/j.tree.2011.03.024.

Sayre, N. F. 2005. "Ecological and Geographical Scale: Parallels and Potential for Integration." Progress in Human Geography 29 (3):276-90. https://doi.org/10.1191/0309132505ph546oa.

Scheiner, S. M., S. B. Cox, M. Willig, G. G. Mittelbach, C. Osenberg, and M. Kaspari. 2000. "Species Richness, Species-Area Curves and Simpson's Paradox." Evolutionary Ecology Research 2:792-801.

Schlup, B. M., and H. H. Wagner. 2008. "Effects of Study Design and Analysis on the Spatial Community Structure Detected by Multiscale Ordination." Journal of Vegetation Science 19 (5):621-32. https://doi.org/10.3170/2008-8-18421. 
Shipley, B., and P. A. Keddy. 1987. "The Individualistic and Community-Unit Concepts as Falsifiable Hypotheses.” Plant Ecology 69 (1):47-55. https://doi.org/10.1007/bf00038686.

Tansley, A. G. 1935. "The Use and Abuse of Vegetational Concepts and Terms." Ecology 16:284-307.

Tichý, L., M. Chytrý, M. Hájek, S. S. Talbot, and Z. Botta-Dukát. 2010. “OptimClass: Using Species-to-Cluster Fidelity to Determine the Optimal Partition in Classification of Ecological Communities." Journal of Vegetation Science 21 (2):287-99. https://doi.org/10.1111/j.1654-1103.2009.01143.x.

Tichý, L., M. Chytrý, and P. Šmarda. 2011. "Evaluating the Stability of the Classification of Community Data." Ecography 34 (5):807-13. https://doi.org/10.1111/j.16000587.2010.06599.x.

Tischendorf, L. 2001. "Can Landscape Indices Predict Ecological Processes Consistently?" Landscape Ecology 16 (3):235-54. http://dx.doi.org/10.1023/A:1011112719782.

Turner, M. G. 1989. "Landscape Ecology: The Effect of Pattern on Process." Annual Review of Ecology and Systematics 20 (1):171-97. https://doi.org/doi:10.1146/annurev.es.20.110189.001131.

Van Den Boogaart, K. G., and R. Tolosana-Delgado. 2008. “'compositions”: A Unified R Package to Analyze Compositional Data." Computers and Geosciences 34 (4):320-38. https://doi.org/10.1016/j.cageo.2006.11.017.

Van Der Maarel, E. 1975. “The Braun-Blanquet Approach in Perspective.” Plant Ecology 30:213-19.

Van Der Maarel, E. 1979. "Transformation of Cover-Abundance Values in Phytosociology and Its Effects on Community Similarity." Plant Ecology 39 (2):97-114. https://doi.org/10.1007/bf00052021.

Vendramin, L., R. J. G. B. Campello, and E. R. Hruschka. 2010. "Relative Clustering Validity Criteria: A Comparative Overview." Statistical Analysis and Data Mining 3 (4):209-35. https://doi.org/10.1002/sam.10080.

Whittaker, R. 1962. "Classification of Natural Communities." The Botanical Review 28 (1):1-239. https://doi.org/10.1007/bf02860872.

Wilcoxon, F. 1945. "Individual Comparisons by Ranking Methods." Biometrics Bulletin 1 (6):80-83. https://doi.org/10.2307/3001968. 
Wildi, O. 2010. Data Analysis in Vegetation Ecology. West Sussex, UK: WileyBlackwell.

With, K. A., and A. W. King. 1997. "The Use and Misuse of Neutral Landscape Models in Ecology.” Oikos 79 (2):219-29. https://doi.org/10.2307/3546007.

Wu, H., and Z.-L. Li. 2009. "Scale Issues in Remote Sensing: A Review on Analysis, Processing and Modeling." Sensors 9 (3):1768-93. https://doi.org/10.3390/s90301768.

Wu, J. 1999. "Hierarchy and scaling : Extrapolating information along a scaling ladder." Vol. 25. Ottawa, ON, CANADA: Canadian Aeronautics and Space Institute.

- 2004. "Effects of Changing Scale on Landscape Pattern Analysis: Scaling Relations." Landscape Ecology 19 (2):125-38. http://dx.doi.org/10.1023/B:LAND.0000021711.40074.ae.

Wu, J., and J. L. David. 2002. "A Spatially Explicit Hierarchical Approach to Modeling Complex Ecological Systems: Theory and Applications.” Ecological Modelling 153 (1-2):7-26. https://doi.org/10.1016/S0304-3800(01)00499-9.

Wu, J., W. Shen, W. Sun, and P. T. Tueller. 2002. "Empirical Patterns of the Effects of Changing Scale on Landscape Metrics.” Landscape Ecology 17 (8):761-82. http://dx.doi.org/10.1023/A:1022995922992. 


\section{CHAPTER II}

QUANTITATIVE SPATIAL UPSCALING OF CLASSIFICATION SYSTEMS

USING A MULTI-DIMENSIONAL GRID POINT CLASSIFIER - REAL LANDSCAPE

\section{APPLICATIONS}

\section{INTRODUCTION}

Spatially explicit models of landscape dynamics as a response to naturally occurring environmental trends and disturbances or to management practices have their advantages over spatially implicit models (DeAngelis and Yurek 2017), but they require the detection of spatially explicit change at adequate spatial and temporal resolutions. Detection and monitoring of land cover are common applications of remote sensing. Reliable interpretation of changes in spectral-reflectance patterns, either as they relate to biophysical parameters of the land surface or as changes in categorical land-cover classes depends on the accurate identification of land cover at the spatial, temporal and thematic precision at which changes are modeled. Often the spatially explicit models of change patterns integrate datasets that have been acquired and/or interpreted at different spatial scales and thus require reconciliation of scales by either upscaling the higher or downscaling the lower resolution data.

For instance, the relationships between spectral reflectance patterns of electromagnetic radiation within the instantaneous field of view (i.e., pixel) recorded at a remote sensor and the biophysical parameters they relate to, depend on the heterogeneity of the surface area covered by a single pixel. For landscapes that display high heterogeneity in cover types relative to the spatial resolution of the remote sensor from which biophysical parameters are derived, cover type of the pixel oversimplifies or misrepresents large 
portions of the actual cover types, which leads to large errors and uncertainty in the estimation of biophysical parameters. Complexities of spatial heterogeneity and reliable estimation of biophysical parameters using remotely sensed data have been identified and described for a suit of parameters and applications (Jacob and Weiss 2014; Liu, Hiyama, and Yamaguchi 2006; Lu 2006). For instance, Leaf Area Index (LAI) which estimates green leaf area per unit ground, and Fraction of Photosynthetically Active Radiation (FPAR), two important biophysical variables in ecosystem productivity models, rely on prior knowledge of land cover, biome or vegetation type (Ganguly et al. 2012; Le Maire et al. 2012; Steltzer and Welker 2006; Zhao et al. 2016). Lotsch et al. (2003) demonstrated the sensitivity of LAI and FPAR to land-cover class information, and heterogeneity of vegetation types within a pixel affects LAI estimates in a non-linear fashion (Garrigues et al. 2006). Tian et al. (2002) showed that LAI errors at coarse resolution are inversely related to the proportion of the dominant land cover in a pixel and that large errors were introduced when the woody component made up only a small proportion of otherwise non-woody pixels. Consequently, as the knowledge of mixed-pixel compositions is limited, the error and uncertainty of estimated LAI is high. However, the most common aggregation method for categorical vegetation maps to match the scale at which LAI and FPAR are generated is the majority or more precisely the plurality rule, which assigns the most common class to the scaled unit, regardless of how low that majority is. Plurality-rule aggregated land-cover maps that have much higher spatial resolution than remotely sensed datasets that are used for estimation of biophysical responses of ecosystems introduce large errors and uncertainty of estimates. Therefore, scaling of land-cover that maintains more precise plant community information 
could reduce error and uncertainty of biophysical parameter estimates from moderateresolution remotely sensed data.

Another application where scaling of land-cover information is required is the modeling of land-cover change across long temporal extents. For a specific geographic region the classification schemes that capture frequent occurrence and co-occurrence patterns of classes vary with scale. Spatial and spectral resolution of a remote sensor determine adequacy of a sensor to differentiate the classes of a classification scheme. Availability of adequate remotely sensed data, that are available at each time step thus dictate the spatial and thematic resolutions at which maps can be derived. As the spatial resolution of remote sensors increases over time, mixed-pixel classes can be represented by pure pixels of their constituent class components resulting in land-cover maps with increasing spatial and thematic precision. For instance, the most extensive archive of remotely sensed data used in the production of land-cover and land-cover change maps is the Landsat Program data repository. Spatial resolution of the multi-spectral data of Thematic Mapper (TM), Enhance Thematic Mapper (ETM) and the Operational Land Imager (OLI) is limited to $30 \mathrm{~m}$, the resolution at which Landsat data are distributed. Since the early 2000s, multi-spectral datasets with high spatial resolution have increasingly become available. The data range from 10-20 m resolution in the case of the European Space Agency's Sentinel-2 data, to less than $5 \mathrm{~m}$ resolution for commercial and private satellites like Digital Globe's WorldView-2 and -3, or Satellite Pour l'Observation de la Terre (SPOT) 5 and 6 data products. Airborne multi- and hyper-spectral sensors mounted on manned or unmanned aerial platforms even allow for mapping at the sub-meter resolution. Combining categorical land-cover maps that were derived at varying spatial 
resolutions requires a reliable scaling algorithm that retains as much information of the higher resolution as possible, while it generates a representative classification scheme that is valid at the lower spatial resolution. The high-information scaled class labels can then be applied to the lower resolution remotely sensed data.

Hence, assessing land-cover change or modeling biophysical response of heterogeneous landscapes across different spatial scales requires (1) consistent scaling of categorical information to lower resolutions and (2) the reliable detection of the lower resolution, scaled, land-cover information from remotely sensed data. Upscaling of categorical maps aggregates information of co-occurrence patterns of class abundances to a coarser resolution (i.e., the spatial scale of analysis). As information of multiple original map objects (i.e., pixels, grid cells) of the initial detection resolution or scale are aggregated, information is generalized and lost. Since the most commonly applied scaling algorithm for categorical data is the majority-rule algorithm, only the original classes of the highresolution map are considered and scaled class labels are assigned on the basis of plurality resulting in huge loss of information as the scale factor increases. To reduce information loss, a scaling algorithm needs to account for scale-specific mixed classes that are frequently encountered across the landscape at the coarser scales.

Limiting information loss, while generating scale-appropriate classification schemes when scaling categorical grid-based data, is the strength of the MDGP-scaling algorithm (Chapter 1). For simulated landscapes with known properties, it has been demonstrated that the $M D G P$-scaling algorithm delivers consistent and reproducible scaling results in terms of information retention (IR) and class-label fidelity (CLF) for a wide range of landscapes. The parameters that control the scaled classification scheme are scale factor, class-label 
precision and minimum representativeness of a scaled class across the larger landscape. Scale factor is determined by the ratio of the lower resolution data to that of the higher resolution. Class-label precision and representativeness across the larger landscape are determined by the user. Considerations that enter into the decision-making for these two parameters are how much detail of the original location-specific information is to be retained and what minimum representativeness of a class across the landscape is desired. In the context of detecting the scaled classes using remote-sensing methods, an additional criterion in the selection of the scaling parameters is the reliable detection of scaled classes from spectral data with high accuracies. Since the two user-defined parameters that drive the scaled classification scheme are class-label precision and landscape-level class representativeness, the effect of these parameters on classification accuracy were evaluated.

On the basis of simulation results presented in Chapter 1, the following relationships between class-label precision and its effects on IR and CLF were established:

(1) Information retention and class count increase with increasing class-label precision.

(2) Class-label fidelity generally decreases with increase in class-label precision.

Adding landscape-level representativeness to the equation, it is expected that:

(3) Information retention decreases when the constraint of landscape representativeness increases, since small classes that increase label precision are removed and grid cells are assigned to classes with less precise and less representative labels.

(4) Class-label fidelity increases with the removal of small classes that do not occur frequently across scaling results.

With the introduction of classification accuracy, two interactions regarding information retention are expected to develop. 
(5) Since class-label precision increases IR, and, therefore, more clearly associates defined thematic classes to spectral classes (signatures), it is expected that separability between classes increases, leading to increased classification accuracy. However, as the number of thematic classes also increases with class-label precision, the chance probability for class confusion increases as well, which reduces accuracy and algorithm confidence.

(6) As the minimum class-representativeness threshold increases, class count decreases, which results in a reduced class confusion and higher classification accuracy. However, as class count decreases, IR at the grid cell level is reduced and grid cells that are further from the nominal class label increase the thematic heterogeneity of the mixed class, and with it, spectral variability, which in return is expected to reduce classification accuracy.

These interactions of class-label precision and class representativeness and their effects on classification accuracy from remotely sensed spectral reflectance data were addressed in this study.

\section{METHODS}

Effects of class-label precision and landscape representativeness, the scaling parameters of the $M D G P$-scaling algorithm, on landscape-level information retention and class separability from remotely sensed, multi-spectral reflectance data were evaluated for two natural landscape types within the greater Everglades ecosystem (FL, USA) (Fig. 2.1). Plant communities for these two landscapes were mapped from WorldView-2 (WV-2; Satellite Imaging Corp., Houston, TX) multi-spectral data at a spatial resolution of $2 \mathrm{~m}$. The resulting landscapes were then scaled to $30 \mathrm{~m}$, the resolution of multi-spectral Landsat 
satellite data. With a scale factor of 15 , relative abundances of classes for $225 \mathrm{WV}-2$ grid cells ( $15 \times 15)$ were generated for each $30 \mathrm{~m}$ grid cell.

Applying the $M D G P$-scaling algorithm in a full factorial design of five options for each of the two scaling parameters, class-label precision and landscape representativeness, to the relative class abundances for each $30 \mathrm{~m}$ grid cell resulted in 25 scaled maps and their associated scale-specific classification schemes. Effects of class-precision and landscape representativeness thresholds on information retention and scaled class-label fidelity were evaluated for 10 random origins of each of the 25 landscapes. Classification accuracy of scaled classes from Landsat data was evaluated for the realized Landsat grid.

\section{Study Areas}

The two landscapes for which the scaling and detection analysis was conducted were (1) a healthy ridge-and-slough patterned landscape within southern Water Conservation Area 3A (WCA3A) and (2) a degraded sawgrass dominated wet prairie in Northeast Shark River Slough (NESRS) (Fig. 2.1). Classification schemes for both regions were developed to capture the common plant communities that can be recognized at the $2 \mathrm{~m}$ resolution of the WV-2 data.

\section{Water Conservation Area $3 A$ - Ridge and Slough}

The ridge-and-slough landscape of WCA3A is characterized by alternating deeper sloughs that are dominated by submerged aquatic, floating broadleaved and emergent graminoid freshwater species (Fig. 2.2). The most common slough species in this region is Nymphaea odorata, which forms dense mats of floating broadleaf carpets and is often accompanied by different species of Utricularia and floating mats of periphyton. The 
elevated ridges that separate the sloughs are dominated by sawgrass (Cladium jamaicense), and in the higher elevations by woody shrubs and various tree species (Fig. 2.2).

The 2-m, scale-specific, classification scheme was composed of eight classes including aquatic submerged, broadleaved floating, graminoid and broadleaved emergent vegetation plus shrubs and trees (Tbl. 2.1). Two areas of $1 \mathrm{~km}^{2}$ each were selected for this landscape, because very high resolution aerial photography had been acquired by a fixedwing unmanned aerial system in August of 2012 for those areas (Zweig et al. 2015). The aerial photography, in combination with field visits at the time of acquisition, provided the basis for the 2-m-scale classification scheme, the digitization of training samples and a design-based accuracy assessment of the mapped community classes.

\section{North-East Shark River Slough - Human Induced Wet Prairie}

The second landscape, a sawgrass dominated wet prairie in NESRS, is a degraded, former ridge-and-slough landscape that experienced decades of altered hydrological regimes, causing a reduction in topographic relief (Larsen et al. 2011; McVoy et al. 2011). As a consequence, the slough communities transitioned into remnant shallow depressions that are dominated by sedges and rushes forming distinct patches within a matrix of sawgrass-dominated communities (Fig. 2.3). These remnant patches are dominated by mixed short-graminoid species of the genera Eleocharis, Panicum, and Rhynchospora. The northern edge of Everglades National Park (ENP) is bordered by Tamiami Trail, a main traffic artery connecting the Florida east coast to its west coast. The construction of Tamiami Trail in the early 1920s bisected the Everglades watershed. Until the recent construction of a bridge, raising the road just north of NESRS, culverts connected the flow of water between a canal parallel and North of Tamiami Trail and ENP. Prior to raising the 
road, the input of nutrient enriched water at the culverts under the road led to the development of shrubby and woody plant communities that formed concentric semi-ellipses of stratified plant communities centered on the culverts; these expanded southward and laterally from the northern border of ENP (Fig. 2.3) into the graminoid-dominated marsh.

The classification scheme for NESRS consisted of 14 community classes: seven included graminoid vegetation; two, broadleaved species; four, shrub and tree components; and two were the non-vegetation classes, water and peat (Tbl. 2.2). This $4.2 \mathrm{~km}^{2}$ study area in the NESRS region, a landscape that exposed a large variety of human-induced plant community co-occurrence patterns, presented an opportunity to test the MDGP-scaling algorithm.

\section{Plant Community Maps}

The high-resolution plant communities that served as the basis for the scaling evaluation were mapped from bi-seasonal WV-2 data at a $2 \mathrm{~m}$ spatial resolution. The vegetation map for WCA3A was delineated from wet ground-condition data acquired on October 20, 2012 and dry ground-condition data from May 5, 2011. Reference data for algorithm training samples were digitized from aerial photography acquired in 2012 by an unmanned aerial system (Zweig et al. 2015). Satellite data for the NESRS map had been acquired on November 6 and 9, 2010 for the wet conditions and on May 6, 2013 for the dry ground conditions. Ortho-rectified and stereo color-infrared aerial photography of 2009 and field-acquired reference data of multiple reconnaissance helicopter flights that were conducted between 2012 and 2014 were used to inform the digitization of training data for all communities. 
All WV-2 satellite scenes were ortho-rectified using the ortho-rectification module in ENVI (Exelis Visual Information Solutions, Boulder, Colorado) applying the rational polynomial coefficients (RPC) that were provided by Digital Globe with each image. After sensor-specific radiometric calibration, atmospheric correction was performed using the Fast Line-of-sight Atmospheric Analysis of Hypercubes (FLAASH) module in ENVI (Exelis Visual Information Solutions, Boulder, Colorado).

Bi-seasonal signatures were extracted for all training samples and cross-validated supervised classification evaluation was performed by applying the random forest algorithm (Breiman 1984). The classifiers were applied to the spectral signatures of each study area to generate the maps for WCA3A (Fig. 2.2) and NESRS (Fig. 2.3). Design-based overall accuracy for the regional maps was estimated from stratified random samples. Accuracy was estimated with a $95 \%$ confidence at $91.2 \%$ for WCA3A and $89.2 \%$ for NESRS (Gann, Richards, and Sadle 2015).

\section{Scaling Parameter Evaluation - Class-Label Fidelity and Information Retention}

The correlations between CLF and IR and their dependencies on scaling parameters were evaluated in a full factorial design for landscape representativeness of 1, 510,15 and 20 percent and class-label precisions of 1, 2, 3, 4, and 5 parts. Monotypic classes that were below the landscape threshold were retained in the scaled classification scheme, since they maintain high information retention and are expected to generate pure spectral signatures with high detection probability and accuracy. To account for random error related to arbitrary grid origin,tenten $30 \mathrm{~m}$ grids with random origins were generated and relative abundance of communities was tabulated from the WV-2 derived map for each grid cell of each of the 10 random grids. Each of the 10 cell-level relative abundance datasets were 
scaled 25 times applying the MDGP-scaling algorithm with the each of the 25 parameter combinations. For each of the 250 scaling results, mean IR was calculated from grid-celllevel IR across all cells of the landscape. Class-label fidelity for each model was estimated with two parameters, the mean probability of a class to occur across the 10 random grid scaling results $\left(\mathrm{CLF}_{\mathrm{m}}\right)$, and the proportion of classes that had a recurrence probability of one $\left(\mathrm{CLF}_{\mathrm{p}}\right)$.

Interactions of $\mathrm{CLF}_{\mathrm{m}}$ and IR were plotted and significance of differences for label precision by representativeness thresholds was tested with a pairwise-paired Wilcoxon signed-rank test, where data were paired by random origin iteration. Optimal scaling parameter solutions for each landscape were identified with an index (OSI) that weighted per-class IR above a user-defined minimum-expected threshold multiplied by the CLF parameters.

$$
O S I=C L F_{m} * C L F_{p} *\left(\frac{I R-I R_{\text {min }}}{\text { class }}\right)
$$

Information retention above the expected minimum $I R_{\min }$ was normalized to per-class IR gain (Eq. 2.1) above the minimum to only give credit to models that reached the minimum expected information retention. The optimal solution model was determined by the maximum OSI across all compared models.

Scaling Parameter Evaluation - Spectral Detection Accuracy

Spectral detection of scaled classes was evaluated for Landsat Thematic Mapper (TM) multi-spectral reflectance data. Landsat 5 TM data acquired on December 25, 2010, was used for the NESRS map and an image from November 11, 2011, for WCA3A. Landsat data used for the two regions were atmospherically corrected using the FLAASH module in 
ENVI (Exelis Visual Information Solutions, Boulder, Colorado). For each study area scaled landscapes were generated for the realized $30 \mathrm{~m}$ grid outline specific to the Landsat scene path 015 row 042 (World Reference System 2). The MDGP-scaling algorithm was applied to the relative class abundances for the Landsat grid using the same set of 25 models of all combinations of the five class precisions ( 1 to 5 ) and the five representativeness thresholds $(1,5,10,15$ and 20). Information retention of all grid cells was averaged for each model and scaled class labels of each grid cell for each of the 25 models were joined with the spectral reflectance data of the corresponding pixel of the processed Landsat TM reflectance data.

Overall accuracy was used to evaluate spectral detectability and separability between classes for each of the 25 scaling models. Overall and class-specific spectral detection accuracies for each of the 25 scaled maps was estimated from the full census of grid cells within each study area using a 10-fold cross-validated classification procedure, when applying the random forest algorithm (Breiman 1984) to each of the 25 full-census training sets. The number of trees was set to 200. To determine the optimal number of randomly selected features at each node, parameter tuning as implemented in the "caret" package (Kuhn 2016) was employed. The "mtry" parameter was evaluated for a range of two to six features, the number of features in the TM dataset.

Since accuracy of class labels increases when the number of classes is low and when labels are coarse or vague, accuracy of categorical maps has to be considered in the context of precision. As class-label precision and class count increase, misclassifications are more likely, purely attributable to chance. Hence, a tradeoff exists between class-label precision and accuracy. Consequently, as class-label precision increases, and with it the number of 
classes in the classification scheme, spectral separability is expected to decrease, because more precisely defined mixed classes are expected to resemble each other spectrally. However, as class-label precision increases, the more likely it is that partial components of the assigned scaled class labels match parts of the actual class label.

A concept that accounts for less severe misclassifications is to give partial credit for labels by weighting the label errors (Cohen 1968). The weight matrix is then applied to the confusion matrix, generating class accuracies with partial-credit weights (Rossiter 2004). Applying partial-credit weights to the multi-dimensional class labels generated by the $M D G P$-scaling algorithm is straightforward, since class percentages are included in the class-label definitions. The weights for the weighted adjustment of the confusion matrix can be directly informed by the class-label discrepancy at the class-label level or even at the pixel level. The portions of the partially matching class labels were used to calculate the weights of the weight matrix. Diagonal elements of the weight matrix were 1 , because all label components match $100 \%$ and the off-diagonal elements were between 0 and 1 , with 0 indicating no agreement of any label component and 1 representing full agreement. The weight matrix calculated for partially matching class labels was calculated with equation 2.2 .

$$
W_{c k}=\sum_{i=1}^{n} p \operatorname{Min}(c i, k i)
$$

Equation 2.2

The weight $\mathrm{W}$ for class combination $c$ and $k$ is the sum of the minimum proportions $p$ Min of each matching label component $i$. For instance, the trivial case of a two-class classification system of the high-resolution reference map that has classes A and B and that is scaled with a class-label precision of $50 \%$ (two parts) theoretically results in a scaled 
three-class classification scheme with classes A100, A50_B50, and B100. The weight for the predicted and referenced class combination A100 and A50_B50 is 0.5, the sum of the minimum proportions of each component, which is 0.5 for class component $\mathrm{A}+0$ for component B. All nine weights of the $3 \times 3$ partial-agreement matrix of this example between all classes are 1, 0.5, 0, 0.5, 1, 0.5, 0, 0.5 and 1 (Eq. 2.2).

Overall accuracy and class-specific omission and commission errors were calculated on the basis of a partial credit for matching components of the detailed class labels. Applying the weights to the confusion matrix, overall (Eq. 2.3) and class-specific user's (Eq. 2.4) and producer's (Eq. 2.5) accuracies and their 95\% confidence intervals were calculated (Rossiter 2004).

$$
A_{o w}=\sum_{i=1}^{r} \sum_{j=1}^{r} w_{i j} * p_{i j}
$$

$A_{o w}$ is the weighted overall accuracy, $w_{i j}$ are the weights calculated with equation 1 , and $p_{i j}$ are the class proportions of the $i$ th predicted row counts and the $j$ th reference class column.

$$
A_{u w}=\frac{1}{p_{i+}} * \sum_{j=1}^{r} w_{i j} * p_{i j}
$$

$A_{u w}$ is the weighted user's accuracy, $w_{i j}$ are weights, and $p_{i j}$ are class proportions of the $i$ th mapped class row and $j$ th reference class column.

$$
A_{p w}=\frac{1}{p_{+j}} \sum_{i=1}^{r} w_{i j} * p_{i j}
$$

$A_{p w}$ is the weighted producer's accuracy, $w_{i j}$ are weights, and $p_{i j}$ are class proportions of the $i$ th mapped class row and $j$ th reference class column. For all 25 scaled landscapes per 
study area, weighted overall accuracies, information retention, and class-label fidelity were used to evaluate and select optimal class-label precision and representativeness parameters.

The trade-offs that have to be negotiated in the case of spectral detection from a realized grid are class-detection accuracy, information retention and representativeness of the scaled classes of a realized grid across the landscape. An index was developed to select the optimal scaling solution considering class-detection accuracy $\left(O S I_{a}\right)$, defined as

$$
O S I_{a}=C L F_{p} *\left(\frac{I R-I R_{\text {min }}}{\text { class }}\right) * A_{o w}
$$

$C L F_{p}$ is the ratio of scaled classes for the realized grid (i.e., WRS-2 of Landsat) when compared to the classes generated from the scaling results of 10 random grid origins. As $C L F_{p}$ increases, the more likely it is that the scaled classes for the current realized grid are representative classes for random locations across the landscape at the scale of interest. Information retention above the expected minimum $\left(I R_{\min }\right)$ was normalized to per-class IR gain above the minimum. $A_{o w}$ is the weighted overall accuracy. The optimal model solution is determined by the maximum of $O S I_{a}$ across all evaluated models. Final maps were generated for optimal scaling solutions that provided the highest information content, given the detectability of the classes from the Landsat reflectance data.

Scaling and spectral detection analysis were performed in R (R Core Team 2016) making extensive use of packages "raster" (Hijmans and van Etten 2010), "rgdal" (Bivand, Keitt, and Rowlingson 2013), "compositions" (Van Den Boogaart and Tolosana-Delgado 2008), and "caret" (Kuhn 2016). Data processing was performed with the highperformance-computing cluster (HPCC) of the Instructional \& Research Computing Center (IRCC) at Florida International University. 


\section{RESULTS}

Interaction effects of the scaling parameters class-label precision and landscape representativeness on information retention and class-label fidelity were similar but not uniform across the two landscape types. Results for scaling and classification accuracy are presented by study area.

\section{WCA3A: Information Retention and Class-Label Fidelity}

Scaling the eight-class plant community map of WCA3A for 10 random origin grids with $30 \mathrm{~m}$ spatial resolution, applying the MDGP-scaling algorithm for the 25 combinations of five class-precisions and five representativeness thresholds produced 250 scaled landscapes and associated scale-specific class schemes. Evaluating the effects of class-label precision and representativeness thresholds for the 250 scaled landscapes confirmed the expected increase in scaled class count and mean IR $(p<0.05)$ with increasing class-label precision for minimum class representativeness across the landscape set to $1 \%$, but increase in IR diminished with increasing class-label precision (Fig. 2.4, Tbl. 2.3). As class representativeness threshold increased to 5 and $10 \%$, the increase of IR with increasing class-label precision diminished to a point where no significant increases for class-label precisions greater than four parts $(25 \%)$ were observed. As representativeness thresholds increased to 15 and $20 \%$, significant IR increase was observed only for label precisions below three parts (33\%) (Fig. 2.4, Tbl. 2.3).

Class-label fidelity generally decreased with increase in class-label precision and representativeness. However, exceptions to the general trend indicated better-than-expected class-label fidelity when precision exceeded $50 \%$ and minimum representativeness increased above $10 \%$ (Tbl. 2.3). Setting the minimum expected IR threshold to $60 \%$, (Tbl. 
2.3), a class-label precision of $25 \%$ with a representativeness threshold of $10 \%(\mathrm{OSI}=0.73)$ or a $33 \%$ class-label precision with a landscape representativeness of $15 \%(\mathrm{OSI}=0.66)$ scored high on the OSI. The 33\%-precision solution on average yielded seven $(S D=0.67)$ scaled classes, with an average IR of $73.5 \%(S D=1.43 \%)$ across the landscape and a mean probability of class-label recurrence of 0.78 , (Fig. 2.4, Tbl. 2.3) with $44 \%$ of classes recurring with a probability of 1 (Tbl. 2.3). The $25 \%$ class-label precision solution produced on average $7.9(S D=0.74)$ scaled classes, which on average retained $77.7 \%(S D=0.73 \%)$ of information and had a mean probability of class-label recurrence of 0.72 (Fig. 2.4, Tbl. 2.3 ), with $45 \%$ of classes re-occurring with a probability of 1 (Tbl. 2.3). In both cases, the class-label fidelity was higher than expected, which increased the optimal scale index.

\section{WCA3A: Spectral-Detection Accuracy}

Scaling the landscape to the specific realized grid of Landsat, IR on average was $1.1 \%(S D=2.33 \%)$ greater than the mean IR across the 10 random origin grids. Overall accuracy ranged from $66.6 \%$ for majority rule with a $1 \%$ class representativeness to $78.2 \%$ (95\% CI: $76.6 \%-79.8 \%$ ) for a $20 \%$ class-label precision and a minimum landscape representativeness of $15 \%$ for each of the five classes the classification scheme produced (Fig. 2.5, Tbl. 2.4).

All scaling solutions with a 1-part or 100\% class-label precision had a mean IR of less than $65 \%($ Mean $=61.9 \% ; S D=0.5 \%)$, which was significantly lower than the MDGPscaled solutions for the two- to five- part label precisions, and produced significantly lower overall accuracies than the corresponding multi-part solutions $(p<0.05)$. The three 2-part (50\%) class precision models that were above $70 \%$ overall accuracy were those with representativeness thresholds of $10 \%$ and greater (Fig. 2.5, Tbl. 2.4). Comparing the three 
solutions showed that the classification schemes were identical and that the differences in accuracy were minor (Tbl. 2.4).

Adding the spectral detection accuracy to the optimal scaling index provided the same scaling solutions as those identified by the OSI. The $33 \%$ class-label precision with a $15 \%$ representativeness threshold produced a classification scheme with 8 classes, an IR of $74.2 \%$ and a class ratio of realized Landsat grid to random origin class solutions of 0.89 (Tbl. 2.4). The overall classification accuracy was $73.9 \%$ (95\% CI: $72.1 \%-75.6 \%$ ), with an $\mathrm{OSI}_{\mathrm{a}}$ of 1.166 (Fig. 2.5, Tbl. 2.4). The second highest $\mathrm{OSI}_{\mathrm{a}}$ was 1.122 scored by the $25 \%$ class-label precision and 10\% representativeness threshold model (Tbl. 2.4). This solution also produced eight scaled classes, retained a slightly higher IR of $76.4 \%$ and had a higher classification accuracy of $75.3 \%(95 \% C I: 73.6 \%-77.0 \%)$, with a class-label count ratio of 0.72 (Fig. 2.4, Tbl. 2.3).

The maps for the two optimal solutions indicate that only the $33 \%$ class-label precision solution maintained the shrub/tree label in of the scaled classes (Tbl. 2.5). This solution was selected as the best-scaled map for a minimum requirement of a $60 \%$ information retention when compared to the original high-resolution input map. This solution had eight classes of which four were monotypic input classes and the other four were mixed classes (Tbl. 2.5). Two of the high-resolution community classes, "Aquatic Submerged" and "Shrub-Tree", which accounted for $2.58 \%$ cover of the high resolution map (Tbl. 2.1) were not maintained in the scaled community class labels. Except for "trees" all class names were included in other mixed class names (Tbl. 2.5). The scaled map and its associated location-specific information retention map are presented in Figure 2.5, and the spectrally classified map and location-specific classifier confidence in Figure 2.6. 
NESRS: Class-Label Fidelity and Information Retention

Results for NESRS were similar to those for WCA3A. The original high-resolution map of NESRS had 14 plant community classes, six more than the WCA3A map. Applying the MDGP-scaling algorithm for all 25 class-label precision and representativenessthreshold combinations confirmed the expected increase in class number and mean IR when class-label precision increased (Fig. 2.8, Tbl. 2.6). With increasing class-label precision, increase in IR diminished, and the differences for consecutive pairwise comparisons became insignificant $(p \geq 0.05$ ), when representativeness was greater than $1 \%$ (Fig. 2.8, Tbl. 2.6). For representativeness of $5 \%$, the four- and five-part label precisions had insignificant differences in IR. As representativeness threshold increased the pairs with insignificant differences increased: for the $10 \%$ representativeness, two- and three-, three- and four-, and four- and five-part solutions; for the 15\% three-, four- and five-part; and for $20 \%$ representativeness also the two- and four-part solutions did not show any difference in IR $(p \geq 0.05)$ (Fig. 2.8, Tbl. 2.6).

Class-label fidelity was significantly higher than for WCA3A. For mean class recurrence probability, the paired comparison by class-label precision and representativeness threshold was $0.15(S D=0.09)$; for class proportion with recurrence probability of 1 , it was $0.24(S D=0.16)$. With a minimum expected IR threshold of $60 \%$, the two solutions, a class-label precision of $33 \%$ with a representativeness threshold of $15 \%$ and a class-label precision of $50 \%$ with representativeness of $5 \%$, both scored an identical OSI of 0.79 , which was the highest when compared to the other 23 models. The $33 \%$ labelprecision solution on average yielded $11.3(S D=0.82)$ scaled classes, with an average IR of $72.7 \%(S D=0.82 \%)$ and a mean probability of class-label recurrence of 0.94 (Fig. 2.8, 
Tbl. 2.6), with $75 \%$ of classes recurring across all random origin iterations (Tbl. 2.6). The $25 \%$ class-label precision solution produced $13.3(S D=0.67)$ scaled classes, which on average retained $74.2 \%(S D=0.88 \%)$ of the information and had a mean probability of class-label recurrence of 0.95 (Fig. 2.8, Tbl. 2.6), with 79\% of classes recurring across all random origin landscapes (Tbl. 2.6).

\section{NESRS: Spectral-Detection Accuracy}

The difference of IR for the Landsat grid scaled maps when compared to the mean IR of the random origins was on average $4.6 \%$ higher $(S D=2.5)$. Overall accuracy ranged from $\sim 69 \%$ for majority-rule solutions to the highest accuracy of $73.2 \%$ (95\% CI: $72.8 \%$ 73.6\%). As in the case of WCA3A, the highest accuracy was achieved for a $20 \%$ classlabel precision and a minimum landscape representativeness of $15 \%$. The number of scaled classes in the classification scheme for this solution was eight (Fig. 2.9, Tbl. 2.7).

All scaling solutions with a 1-part or 100\% label precision had a mean IR of 70.3\% $(S D=0.0 \%)$, which was significantly lower than the $M D G P$-scaled solutions for the twoto five- part label precisions. Accuracy was significantly higher for all multi-part solutions with a class representativeness greater than $5 \%(p<0.05)$ (Fig. 2.9, Tbl. 2.7). For classlabel precisions of $50 \%$ and less, the $15 \%$ and $20 \%$ representativeness thresholds produced identical classification solutions.

Adding spectral-detection accuracy to the optimal scaling index suggests that the $33 \%$ class-label precision with a $10 \%$ class representativeness threshold produced the best scaling result $(\mathrm{OSI}=1.105)$, generating 13 scaled classes that were detected from multispectral Landsat data with an accuracy of 70.7\% (95\% CI: 70.3\% - 71.1\%) (Tbl. 2.7). The information retained for this solution was $80.3 \%$, and the class-label count ratio was 1 , 
indicating that all classes derived for the Landsat-grid were represented in the random origin solutions (Fig. 2.9, Tbl. 2.7).

Scaled community classes for the optimal solution of 33\% class-label precision included three mixed classes and 10 monotypic input classes (Tbl. 2.8). The community types of the original classes that were omitted in the scaled class labels were "Broadleaved Floating", "Tree Hammock", "Water", and "Peat" (Tbls. 2.2 and 2.8). These four classes, however, only accounted for $0.4 \%$ of cover in the original map (Tbl. 2). The small class of "Tree Bayhead" was maintained as a monotypic class with the exact same cover percentage of $0.28 \%$ as the original map and a mean information retention of $82.5 \%(S D=19.6)$. The scaled map and its associated information retention by grid cell are presented in Figure 2.10, and the spectrally classified map with location-specific classifier confidence in Figure 2.11.

\section{DISCUSSION}

Ecological models of ecosystem responses to climate change, management practices and natural disturbance rely on spatially explicit and exhaustive datasets with adequate spatial and temporal resolutions. Remotely sensed datasets that are integrated in these models are often acquired at different spatial resolutions. This discrepancy in spatial resolutions requires upscaling of the high-resolution data or derived products to that of the low-resolution data. Combining low-resolution datasets that have high temporal resolution with the up-scaled products of high spatial but low temporal resolution is more effective when information retention of the scaled product is maximized.

The objective of my study was to evaluate the effects of class-label precision on class-label fidelity and information retention when scaling high-precision vegetation maps of real-world landscapes using the $M D G P$-scaling algorithm and to determine optimal class- 
label precision and class-representativeness thresholds. The second objective was to evaluate the correlation of class-label precision and representativeness of scaled classes to their detectability from remotely sensed data, acquired at a lower spatial resolution than the original scaled map. Results of the scaling analysis for two natural wetland landscapes indicated that optimizing class-label precision and representativeness is possible when implemented in a full factorial evaluation framework. The analysis also demonstrated that precise and representative classes were detectable from low resolution remotely sensed data with acceptable accuracy, and that class-detection accuracy increased when compared to landscapes scaled with the standard majority-rule method.

Differences in information retention for optimal scaling solutions were significantly higher than the trivial majority-rule solutions, regardless of landscape. For both landscapes, intermediate class-label precisions of $33 \%$ were suitable solutions, negotiating information retention, class-label fidelity and class-detection accuracy. While for both landscapes, information retention increased and class-label fidelity decreased with increasing class-label precision, the pattern was observed only for landscape representativeness thresholds below 15 percent (Figs. 2.2 and 2.3).

The trade-offs of information retention, class-label fidelity, and spectral detectability of scaled classes from multi-spectral data indicate that there no single-best solutions exist. Weighting these criteria when selecting the optimal solution is user- and applicationdependent. Class-specific accuracy and classification scheme preferences can be used in the selection of the optimal parameter selection. The presented method provides a framework that integrates the quantitative evaluation of scaling parameter selection and its effects on representativeness of classification systems, information retention at the local 
(pixel) and at the landscape level, and the spectral-detection probabilities of the scaled classes. Applying this method allows for user-specific and preference-optimized solutions where previously no weighting of effects was possible.

The algorithm in the current version requires user-defined values for class-label precision and class-representativeness as a percentage of landscape cover. To simplify parameter selection the user-defined minimum class representativeness thresholds could be implemented as a step-wise class-removal procedure. The feedback of the step-wise removal could be the three criteria, IR, CLF and classification accuracy. Successful implementation of a step-wise class-removal procedure depends on the correlation of location-specific (i.e., grid cell), class-specific, and landscape-level information retention to overall and class-specific classification accuracy, which still needs to be evaluated.

The effects of scaling optimization and parameter selection on the accuracy of categorical vegetation-change detection using the Landsat archive of $30+$ years and to evaluate and estimate gains in estimate precision and reduction of error and uncertainty when estimating biophysical response parameters (e.g., respiration, biomass, LAI) needs to be tested. Interpreting biophysical response parameters over large regions using remotesensing techniques requires not only understanding of the relationship between the variable or parameter of interest in relation to the remotely sensed data, but also the land-cover compositions over the area for which response parameters are estimated. Differences in relative abundance of each vegetation type affects the calibration and performance of models. Biogeochemical models that simulate carbon and nitrogen fluxes between soil, vegetation and the atmosphere often use spatially low-resolution data with the advantage that they have much higher temporal resolution than the datasets that are used to generate 
vegetation maps. The availability of vegetation maps at high spatial resolutions that provide the spatial precision required to separate and distinguish borders of plant communities, assemblages or ecotones of interest can serve as model landscapes from which scaleappropriate vegetation-classification systems can be derived by applying the $M D G P$-scaling algorithm. Other scaling methods that only use input classes of the high-resolution cover maps do not account for class co-occurrence patterns at different spatial scales, and, therefore, reduce information content that can aid in the high-precision estimation of biophysical parameters.

Spatially explicit and exhaustive ecosystem response models that use multi-spectral reflectance data at high temporal resolutions are limited by their low spatial resolution, integrating and interpreting reflectance patterns over large areas of a single response unit (pixel). For instance, combining LAI or FPAR products derived from Moderate Resolution Imaging Spectroradiometer (MODIS) (Knyazikhin et al. 1999; Myneni, Knyazikhin, and Park 2015), which has have a high temporal resolution (i.e., daily) but low spatial resolution (i.e., $500 \mathrm{~m}$ ), with vegetation maps that are the product of high-precision mapping requires downscaling of the vegetation maps. Knowing the approximate relative abundance of vegetation cover types within each response unit (pixel, grid cell) of the lower-resolution data, allows for more precise modeling of response variables (i.e., LAI, FPAR). Hence, scaling high-resolution land-cover maps to match low-resolution data layers of response variables with high scale factors using the $M D G P$-scaling algorithm generates classification schemes that retain more detailed ground cover information, which allows for estimates that are more precise. This analysis demonstrated that detection of scaled classes from lower resolution spectral data was possible and that the evaluation framework facilitates parameter 
selection that optimizes scaling results. Quantifying class-specific and location-specific information retention for the scaled products also enables the estimation of spatially explicit confidence or error at the low-resolution grid cell level and thus error propagation to model results.

Application of the MDGP-scaling algorithm for change detection of land cover can be performed for frequently co-occurring classes. With the increasing availability of highresolution remotely sensed data, high-precision land-cover maps will become more common, but the detection of past changes requires robust and reliable aggregation methods of categorical data. Only when scaling procedures produce representative, scale-appropriate low-resolution land-cover maps with scaled classes that can be detected from low-resolution spectral reflectance patterns will change-detection analysis deliver accurate change patterns with acceptable confidence. 


\section{TABLES}

Table 2.1. Classification scheme of high-resolution plant community map for WCA3A (Fig. 2.2) and mapped class proportions for the study area.

\begin{tabular}{|c|c|c|c|}
\hline Region & Class Abbr & Class Name & Class Prop. (\%) \\
\hline \multirow{8}{*}{ 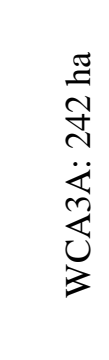 } & $\mathrm{aS}$ & Aquatic Submerged & 1.81 \\
\hline & aS-p-blF & Aquatic Submerged - Periphyton - Broadleaved Floating & 28.16 \\
\hline & blF-aS & Broadleaved Floating - Aquatic Submerged & 10.82 \\
\hline & blFNy & Broadleaved Floating Nymphaea & 10.3 \\
\hline & gM-blE & Graminoid Marsh - Broadleaved Emergent & 18.93 \\
\hline & $\mathrm{gMCl}$ & Graminoid Marsh Cladium & 24.73 \\
\hline & s-gM-blE & Shrub - Graminoid Marsh - Broadleaved Emergent & 4.49 \\
\hline & $\mathrm{s}-\mathrm{t}$ & Shrub - Tree & 0.77 \\
\hline
\end{tabular}

Table 2.2. Classification scheme of high-resolution plant community map for NESRS (Fig. 2.3) and mapped class proportions for the study area.

\begin{tabular}{|c|c|c|c|}
\hline Region & Class Abbr & Class Name & Class Prop. (\%) \\
\hline \multirow{14}{*}{ 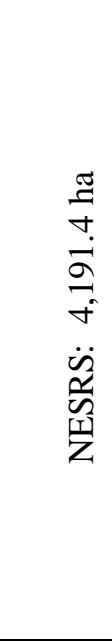 } & $\mathrm{bF}$ & Broadleaved Floating & 0.02 \\
\hline & $\mathrm{gM}-\mathrm{bE}$ & Graminoid Marsh - Broadleaved Emergent & 2.92 \\
\hline & gM_S & Graminoid Marsh Sparse & 7.99 \\
\hline & gM_D & Graminoid Marsh Dense & 2.3 \\
\hline & $\mathrm{gMCl}$ & Graminoid Marsh Cladium & 29.3 \\
\hline & gMCl_D & Graminoid Marsh Cladium Dense & 13.66 \\
\hline & gMCl_S & Graminoid Marsh Cladium Sparse & 35.46 \\
\hline & gMTy & Graminoid Marsh Typha & 2.67 \\
\hline & $\mathrm{sB}$ & Shrub Bayhead & 2.01 \\
\hline & sSa & Shrub Salix & 3.02 \\
\hline & $\mathrm{tB}$ & Tree Bayhead & 0.28 \\
\hline & $\mathrm{tH}$ & Tree Hammock & 0.06 \\
\hline & w & Water & 0.07 \\
\hline & $\mathrm{pt}$ & Peat & 0.25 \\
\hline
\end{tabular}


Table 2.3. WCA3A scaling results for varying class-label precisions (Parts) and landscape representativeness thresholds (Rpr.). prbMn = mean probability of class recurrence across 10 random origin iterations; prp1 = proportion of classes across 10 iterations with recurrence probability of 1 ; cls-Tot $=$ total number of classes across 10 iterations; cls-Mix = number of mixed classes; cls-Mono = number of monotypic classes; clsMn-RO = mean number of classes for iterations; clsSD-RO = standard deviation of class numbers; irMn-LS = mean information retention across all 10 scaled landscapes; irSD-LS = standard deviation of information retention across all 10 scaled landscapes; OSI = Optimal Scaling Index (i.e., credit for information retention greater than $60 \%$ ). Best two solutions are marked in dark grey.

\begin{tabular}{|c|c|c|c|c|c|c|c|c|c|c|c|c|}
\hline \multirow[b]{2}{*}{ Region } & \multirow[b]{2}{*}{ Parts } & \multirow[b]{2}{*}{ Rpr. } & \multicolumn{7}{|c|}{ Class Fidelity } & \multicolumn{2}{|c|}{ Information } & \multirow{2}{*}{$\begin{array}{c}\text { OSI } \\
\text { IR }>60 \%\end{array}$} \\
\hline & & & prbMn & prp1 & $\begin{array}{r}\text { cls } \\
\text { Tot } \\
\end{array}$ & $\begin{array}{r}\text { cls } \\
\text { Mix } \\
\end{array}$ & $\begin{array}{r}\text { cls } \\
\text { Mono } \\
\end{array}$ & $\begin{array}{r}\text { clsMn } \\
\text { RO } \\
\end{array}$ & $\begin{array}{r}\text { clsSD } \\
\text { RO } \\
\end{array}$ & $\begin{array}{r}\text { irMn } \\
\text { LS } \\
\end{array}$ & $\begin{array}{r}\text { irSD } \\
\mathrm{LS} \\
\end{array}$ & \\
\hline \multirow{25}{*}{$\sum_{3}^{\frac{\pi}{3}}$} & 1 & 1 & 1.00 & 1.00 & 7 & 0 & 7 & 7.0 & 0.00 & $57.3 \%$ & $0.45 \%$ & -0.392 \\
\hline & 1 & 5 & 0.89 & 0.71 & 7 & 0 & 7 & 6.2 & 0.63 & $58.0 \%$ & $0.57 \%$ & -0.205 \\
\hline & 1 & 10 & 0.80 & 0.71 & 7 & 0 & 7 & 5.6 & 0.70 & $58.6 \%$ & $0.63 \%$ & -0.147 \\
\hline & 1 & 15 & 0.80 & 0.71 & 7 & 0 & 7 & 5.6 & 0.70 & $58.6 \%$ & $0.63 \%$ & -0.147 \\
\hline & 1 & 20 & 0.80 & 0.71 & 7 & 0 & 7 & 5.6 & 0.70 & $58.6 \%$ & $0.63 \%$ & -0.147 \\
\hline & 2 & 1 & 0.89 & 0.79 & 19 & 12 & 7 & 17.0 & 1.15 & $74.8 \%$ & $0.62 \%$ & 0.613 \\
\hline & 2 & 5 & 0.80 & 0.53 & 15 & 8 & 7 & 12.0 & 0.67 & $72.0 \%$ & $0.53 \%$ & 0.426 \\
\hline & 2 & 10 & 0.76 & 0.40 & 10 & 3 & 7 & 7.6 & 0.97 & $70.0 \%$ & $0.92 \%$ & 0.399 \\
\hline & 2 & 15 & 0.72 & 0.30 & 10 & 3 & 7 & 7.2 & 1.14 & $69.5 \%$ & $0.87 \%$ & 0.285 \\
\hline & 2 & 20 & 0.67 & 0.30 & 10 & 3 & 7 & 6.7 & 0.95 & $67.8 \%$ & $2.07 \%$ & 0.233 \\
\hline & 3 & 1 & 0.89 & 0.82 & 34 & 29 & 5 & 30.1 & 1.20 & $80.5 \%$ & $0.29 \%$ & 0.497 \\
\hline & 3 & 5 & 0.67 & 0.37 & 19 & 14 & 5 & 12.8 & 0.63 & $76.8 \%$ & $0.62 \%$ & 0.327 \\
\hline & 3 & 10 & 0.61 & 0.29 & 14 & 9 & 5 & 8.5 & 0.71 & $74.9 \%$ & $0.95 \%$ & 0.304 \\
\hline & 3 & 15 & 0.78 & 0.44 & 9 & 4 & 5 & 7.0 & 0.67 & $73.5 \%$ & $1.43 \%$ & 0.664 \\
\hline & 3 & 20 & 0.66 & 0.22 & 9 & 4 & 5 & 5.9 & 0.57 & $70.5 \%$ & $1.74 \%$ & 0.259 \\
\hline & 4 & 1 & 0.78 & 0.58 & 52 & 48 & 4 & 40.3 & 1.16 & $83.4 \%$ & $0.20 \%$ & 0.260 \\
\hline & 4 & 5 & 0.67 & 0.35 & 20 & 16 & 4 & 13.4 & 1.26 & $79.7 \%$ & $0.55 \%$ & 0.345 \\
\hline & 4 & 10 & 0.72 & 0.45 & 11 & 7 & 4 & 7.9 & 0.74 & $77.7 \%$ & $0.73 \%$ & 0.732 \\
\hline & 4 & 15 & 0.57 & 0.18 & 11 & 7 & 4 & 6.3 & 0.67 & $75.9 \%$ & $1.57 \%$ & 0.263 \\
\hline & 4 & 20 & 0.54 & 0.22 & 9 & 5 & 4 & 4.9 & 0.32 & $71.6 \%$ & $1.44 \%$ & 0.287 \\
\hline & 5 & 1 & 0.61 & 0.36 & 78 & 75 & 3 & 47.8 & 1.62 & $85.2 \%$ & $0.19 \%$ & 0.116 \\
\hline & 5 & 5 & 0.48 & 0.14 & 29 & 26 & 3 & 13.8 & 1.14 & $80.6 \%$ & $0.76 \%$ & 0.098 \\
\hline & 5 & 10 & 0.57 & 0.07 & 14 & 11 & 3 & 8.0 & 0.82 & $77.6 \%$ & $1.33 \%$ & 0.090 \\
\hline & 5 & 15 & 0.46 & 0.08 & 12 & 9 & 3 & 5.5 & 0.53 & $74.4 \%$ & $1.74 \%$ & 0.100 \\
\hline & 5 & 20 & 0.58 & 0.13 & 8 & 5 & 3 & 4.6 & 0.52 & $72.8 \%$ & $1.65 \%$ & 0.199 \\
\hline
\end{tabular}


Table 2.4. Scaling effects on spectral detection accuracies for WCA3A for varying class-label precisions (Parts) and landscape representativeness thresholds (Rpr.). IR-RL = mean information retention across the landscape for the realized landscape (Landsat grid); clsLS = number of scaled classes; clsLS/clsRO = the ration of scaled classes for the realized landscape to the number of all classes identified across 10 random grid origins; $\mathrm{cvOA}=$ cross-validated overall classification accuracy; OA-CIL and OA-CIU = lower and upper confidence estimates of the overall accuracy; OSIa = Optimal Scaling Index for classdetection accuracy. Best two solutions are marked in dark grey.

\begin{tabular}{|c|c|c|c|c|c|c|c|c|c|}
\hline Region & \multicolumn{2}{|c|}{ Parts Rpr. } & \multicolumn{3}{|c|}{ IR-RL clsLS clsLS/clsRO } & \multirow{2}{*}{ cvOA } & \multirow{2}{*}{ OA-CIL } & \multirow{2}{*}{$\frac{\text { OA-CIU }}{68.5 \%}$} & \multirow{2}{*}{$\begin{array}{c}\text { OSIa } \\
0.247\end{array}$} \\
\hline \multirow{25}{*}{$\sum_{3}^{\frac{\pi}{3}}$} & 1 & 1 & $62.6 \%$ & 7 & 1.00 & & & & \\
\hline & 1 & 5 & $62.3 \%$ & 6 & 0.86 & $67.1 \%$ & $65.3 \%$ & $68.9 \%$ & 0.222 \\
\hline & 1 & 10 & $61.5 \%$ & 5 & 0.71 & $70.0 \%$ & $68.2 \%$ & $71.8 \%$ & 0.151 \\
\hline & 1 & 15 & $61.5 \%$ & 5 & 0.71 & $69.6 \%$ & $67.8 \%$ & $71.4 \%$ & 0.150 \\
\hline & 1 & 20 & $61.5 \%$ & 5 & 0.71 & $70.4 \%$ & $68.6 \%$ & $72.2 \%$ & 0.152 \\
\hline & 2 & 1 & $77.1 \%$ & 16 & 0.84 & $68.3 \%$ & $66.5 \%$ & $70.1 \%$ & 0.615 \\
\hline & 2 & 5 & $75.5 \%$ & 12 & 0.80 & $70.4 \%$ & $68.6 \%$ & $72.2 \%$ & 0.726 \\
\hline & 2 & 10 & $71.3 \%$ & 7 & 0.70 & $72.3 \%$ & $70.5 \%$ & $74.0 \%$ & 0.817 \\
\hline & 2 & 15 & $71.3 \%$ & 7 & 0.70 & $71.8 \%$ & $70.1 \%$ & $73.6 \%$ & 0.812 \\
\hline & 2 & 20 & $71.3 \%$ & 7 & 0.70 & $72.1 \%$ & $70.4 \%$ & $73.9 \%$ & 0.815 \\
\hline & 3 & 1 & $82.8 \%$ & 29 & 0.85 & $69.2 \%$ & $67.4 \%$ & $71.0 \%$ & 0.464 \\
\hline & 3 & 5 & $78.6 \%$ & 12 & 0.63 & $71.5 \%$ & $69.7 \%$ & $73.3 \%$ & 0.698 \\
\hline & 3 & 10 & $76.0 \%$ & 9 & 0.64 & $72.1 \%$ & $70.3 \%$ & $73.8 \%$ & 0.822 \\
\hline & 3 & 15 & $74.2 \%$ & 8 & 0.89 & $73.9 \%$ & $72.1 \%$ & $75.6 \%$ & 1.166 \\
\hline & 3 & 20 & $69.2 \%$ & 6 & 0.67 & $72.9 \%$ & $71.2 \%$ & $74.6 \%$ & 0.749 \\
\hline & 4 & 1 & $85.9 \%$ & 40 & 0.77 & $69.5 \%$ & $67.7 \%$ & $71.3 \%$ & 0.346 \\
\hline & 4 & 5 & $80.2 \%$ & 14 & 0.70 & $72.5 \%$ & $70.8 \%$ & $74.3 \%$ & 0.734 \\
\hline & 4 & 10 & $76.4 \%$ & 8 & 0.73 & $75.3 \%$ & $73.6 \%$ & $77.0 \%$ & 1.122 \\
\hline & 4 & 15 & $75.1 \%$ & 7 & 0.64 & $76.1 \%$ & $74.4 \%$ & $77.7 \%$ & 1.041 \\
\hline & 4 & 20 & $71.0 \%$ & 5 & 0.56 & $74.8 \%$ & $73.1 \%$ & $76.5 \%$ & 0.918 \\
\hline & 5 & 1 & $87.1 \%$ & 47 & 0.60 & $69.8 \%$ & $68.0 \%$ & $71.6 \%$ & 0.242 \\
\hline & 5 & 5 & $80.5 \%$ & 13 & 0.45 & $73.2 \%$ & $71.5 \%$ & $75.0 \%$ & 0.517 \\
\hline & 5 & 10 & $75.2 \%$ & 8 & 0.57 & $78.0 \%$ & $76.4 \%$ & $79.7 \%$ & 0.847 \\
\hline & 5 & 15 & $71.4 \%$ & 5 & 0.42 & $78.2 \%$ & $76.6 \%$ & $79.8 \%$ & 0.741 \\
\hline & 5 & 20 & $68.9 \%$ & 4 & 0.50 & $77.5 \%$ & $75.8 \%$ & $79.1 \%$ & 0.857 \\
\hline
\end{tabular}


Table 2.5. WCA3A class schema and class proportions (Class Prop.) for two good scaling solutions of a 3-part class-label precision with a landscape representativeness of $15 \%$ (top) and a 4-part class-label precision with a representativeness of $15 \%($ bottom $)$. irMn = mean information retention; irSD = standard deviation of information retention.

\begin{tabular}{lccc}
\hline Class Name - 33\% Precision 15\% Representativeness & Class Prop. (\%) & irMn (\%) & irSD (\%) \\
\hline 100 Aquatic Submerged - Periphyton - Broadleaved Floating * & 15.7 & 88.9 & 9.2 \\
\hline $\begin{array}{l}\text { 67 Aquatic Submerged - Periphyton - Broadleaved Floating } \\
\text { 33 Broadleaved Floating Nymphaea }\end{array}$ & 19.9 & 68.0 & 14.7 \\
\hline 100 Broadleaved Floating - Aquatic Submerged * & 9.7 & 65.6 & 19.2 \\
\hline 33 Broadleaved Floating Nymphaea \\
$\begin{array}{l}\text { 33 Graminoid Marsh - Broadleaved Emergent } \\
\text { 33 Graminoid Marsh Cladium }\end{array}$ & 21.8 & 65.1 & 11.8 \\
\hline 100 Graminoid Marsh - Broadleaved Emergent * & & & \\
\hline 100 Graminoid Marsh Cladium* & 5.2 & 69.0 & 11.7 \\
\hline $\begin{array}{l}\text { 67 Graminoid Marsh Cladium } \\
\text { 33 Graminoid Marsh - Broadleaved Emergent }\end{array}$ & 6.6 & 89.5 & 7.2 \\
\hline 100 Shrub - Graminoid Marsh - Broadleaved Emergent * & 18.4 & 81.2 & 11.0 \\
\hline
\end{tabular}

Class Name - 25\% Precision 10\% Representativeness

Class Prop. (\%) irMn (\%) irSD (\%)

\begin{tabular}{|c|c|c|c|}
\hline 100 Aquatic Submerged - Periphyton - Broadleaved Floating * & 13.0 & 89.3 & 16.6 \\
\hline $\begin{array}{l}25 \text { Aquatic Submerged - Periphyton - Broadleaved Floating } \\
25 \text { Broadleaved Floating - Aquatic Submerged } \\
25 \text { Broadleaved Floating Nymphaea } \\
25 \text { Graminoid Marsh - Broadleaved Emergent }\end{array}$ & 12.8 & 62.6 & 17.0 \\
\hline $\begin{array}{l}25 \text { Aquatic Submerged - Periphyton - Broadleaved Floating } \\
25 \text { Broadleaved Floating - Aquatic Submerged } \\
25 \text { Graminoid Marsh - Broadleaved Emergent } \\
25 \text { Graminoid Marsh Cladium }\end{array}$ & 15.9 & 68.6 & 15.0 \\
\hline $\begin{array}{l}75 \text { Aquatic Submerged - Periphyton - Broadleaved Floating } \\
25 \text { Broadleaved Floating Nymphaea }\end{array}$ & 14.0 & 78.1 & 12.4 \\
\hline 100 Broadleaved Floating - Aquatic Submerged* & 7.0 & 74.0 & 14.5 \\
\hline $\begin{array}{l}50 \text { Graminoid Marsh - Broadleaved Emergent } \\
25 \text { Broadleaved Floating Nymphaea } \\
25 \text { Graminoid Marsh Cladium } \\
\end{array}$ & 16.0 & 72.8 & 9.9 \\
\hline 100 Graminoid Marsh Cladium* & 5.0 & 92.4 & 5.1 \\
\hline $\begin{array}{l}67 \text { Graminoid Marsh Cladium } \\
33 \text { Graminoid Marsh - Broadleaved Emergent }\end{array}$ & 16.2 & 82.9 & 10.4 \\
\hline
\end{tabular}


Table 2.6. NESRS scaling results for varying class-label precisions (Parts) and landscape representativeness thresholds $(\mathrm{LS}-\mathrm{Rpr}$.$) . prbMn =$ mean probability of class recurrence across 10 random origin iterations; prp1 = proportion of classes across 10 iterations with recurrence probability of 1 ; clsTot $=$ total number of classes across 10 iterations; clsMix = number of mixed classes; clsMono = number of monotypic classes; clsMn-RO = mean number of classes for iterations; clsSD-RO = standard deviation of class numbers; irMn-LS = mean information retention across all 10 scaled landscapes; irSD-LS = standard deviation of information retention across all 10 scaled landscapes; OSI = Optimal Scaling Index (i.e., credit for information retention greater than $60 \%$ ). Best two solutions are marked in dark grey.

\begin{tabular}{|c|c|c|c|c|c|c|c|c|c|c|c|c|}
\hline \multirow[b]{2}{*}{ Region } & \multirow[b]{2}{*}{ Parts } & \multirow[b]{2}{*}{ Rpr. } & \multicolumn{7}{|c|}{ Class Fidelity } & \multicolumn{2}{|c|}{ Information } & \multirow{2}{*}{$\begin{array}{c}\text { OSI } \\
\text { IR }>60 \%\end{array}$} \\
\hline & & & prbMn & prp1 & $\begin{array}{r}\text { cls } \\
\text { Tot } \\
\end{array}$ & $\begin{array}{r}\text { cls } \\
\text { Mix } \\
\end{array}$ & $\begin{array}{r}\text { cls } \\
\text { Mono } \\
\end{array}$ & $\begin{array}{r}\text { clsMn } \\
\text { RO }\end{array}$ & $\begin{array}{r}\text { clsSD } \\
\text { RO } \\
\end{array}$ & $\begin{array}{r}\text { irMn } \\
\text { LS } \\
\end{array}$ & $\begin{array}{r}\text { irSD } \\
\text { LS } \\
\end{array}$ & \\
\hline \multirow{25}{*}{$\begin{array}{l}\mathscr{2} \\
\frac{\pi}{Z} \\
\frac{\pi}{Z}\end{array}$} & 1 & 1 & 1.00 & 1.00 & 11 & 0 & 11 & 11.0 & 0.00 & $66.4 \%$ & $0.21 \%$ & 0.582 \\
\hline & 1 & 5 & 1.00 & 1.00 & 11 & 0 & 11 & 11.0 & 0.00 & $66.4 \%$ & $0.21 \%$ & 0.582 \\
\hline & 1 & 10 & 1.00 & 1.00 & 11 & 0 & 11 & 11.0 & 0.00 & $66.4 \%$ & $0.21 \%$ & 0.582 \\
\hline & 1 & 15 & 1.00 & 1.00 & 11 & 0 & 11 & 11.0 & 0.00 & $66.5 \%$ & $0.20 \%$ & 0.587 \\
\hline & 1 & 20 & 1.00 & 1.00 & 11 & 0 & 11 & 11.0 & 0.00 & $66.5 \%$ & $0.20 \%$ & 0.587 \\
\hline & 2 & 1 & 0.91 & 0.78 & 23 & 12 & 11 & 20.9 & 0.57 & $77.3 \%$ & $0.50 \%$ & 0.588 \\
\hline & 2 & 5 & 0.95 & 0.79 & 14 & 3 & 11 & 13.3 & 0.67 & $74.2 \%$ & $0.88 \%$ & 0.794 \\
\hline & 2 & 10 & 0.95 & 0.77 & 13 & 2 & 11 & 12.3 & 0.67 & $72.2 \%$ & $0.96 \%$ & 0.722 \\
\hline & 2 & 15 & 0.94 & 0.83 & 12 & 1 & 11 & 11.3 & 0.67 & $70.2 \%$ & $0.69 \%$ & 0.710 \\
\hline & 2 & 20 & 0.94 & 0.83 & 12 & 1 & 11 & 11.3 & 0.67 & $70.2 \%$ & $0.69 \%$ & 0.710 \\
\hline & 3 & 1 & 0.85 & 0.67 & 30 & 20 & 10 & 25.4 & 1.17 & $81.2 \%$ & $0.41 \%$ & 0.471 \\
\hline & 3 & 5 & 0.90 & 0.65 & 17 & 7 & 10 & 15.3 & 0.82 & $78.1 \%$ & $1.50 \%$ & 0.690 \\
\hline & 3 & 10 & 0.92 & 0.62 & 13 & 3 & 10 & 12.0 & 0.94 & $74.1 \%$ & $1.56 \%$ & 0.667 \\
\hline & 3 & 15 & 0.94 & 0.75 & 12 & 2 & 10 & 11.3 & 0.82 & $72.7 \%$ & $0.82 \%$ & 0.793 \\
\hline & 3 & 20 & 0.93 & 0.67 & 12 & 2 & 10 & 11.2 & 0.79 & $72.5 \%$ & $1.02 \%$ & 0.696 \\
\hline & 4 & 1 & 0.78 & 0.62 & 42 & 32 & 10 & 32.7 & 0.95 & $83.9 \%$ & $0.30 \%$ & 0.353 \\
\hline & 4 & 5 & 0.84 & 0.65 & 20 & 10 & 10 & 16.8 & 1.03 & $81.5 \%$ & $0.98 \%$ & 0.699 \\
\hline & 4 & 10 & 0.80 & 0.53 & 15 & 5 & 10 & 12.0 & 0.82 & $76.5 \%$ & $1.47 \%$ & 0.587 \\
\hline & 4 & 15 & 0.73 & 0.50 & 14 & 4 & 10 & 10.2 & 1.32 & $73.0 \%$ & $0.71 \%$ & 0.465 \\
\hline & 4 & 20 & 0.69 & 0.43 & 14 & 4 & 10 & 9.7 & 1.49 & $72.0 \%$ & $1.41 \%$ & 0.367 \\
\hline & 5 & 1 & 0.63 & 0.48 & 61 & 52 & 9 & 38.5 & 1.08 & $85.7 \%$ & $0.19 \%$ & 0.200 \\
\hline & 5 & 5 & 0.60 & 0.36 & 28 & 19 & 9 & 16.8 & 1.03 & $82.5 \%$ & $0.62 \%$ & 0.287 \\
\hline & 5 & 10 & 0.69 & 0.38 & 16 & 7 & 9 & 11.1 & 1.20 & $76.7 \%$ & $1.23 \%$ & 0.391 \\
\hline & 5 & 15 & 0.69 & 0.36 & 14 & 5 & 9 & 9.7 & 0.67 & $73.7 \%$ & $0.93 \%$ & 0.349 \\
\hline & 5 & 20 & 0.67 & 0.36 & 14 & 5 & 9 & 9.4 & 0.70 & $73.2 \%$ & $1.45 \%$ & 0.336 \\
\hline
\end{tabular}


Table 2.7. Scaling effects on spectral detection accuracies for NESRS for varying class-label precisions (Parts) and landscape representativeness thresholds (Rpr.). IR-RL = mean information retention across the landscape for the realized landscape (Landsat grid); clsLS = number of scaled classes; clsLS/clsRO = the ration of scaled classes for the realized landscape to the number of all classes identified across 10 random grid origins; $\mathrm{cvOA}=$ cross-validated overall classification accuracy; OA-CIL and OA-CIU = lower and upper confidence estimates of the overall accuracy; OSIa = Optimal Scaling Index for class detection accuracy. Best solution is marked in dark grey.

\begin{tabular}{|c|c|c|c|c|c|c|c|c|c|}
\hline Region & Parts & Rpr. & IR-RL & clsLS & clsLS/clsRO & cvOA & OA-CIL & OA-CIU & OSIa \\
\hline \multirow{25}{*}{$\begin{array}{l}\mathscr{2} \\
\frac{\alpha}{2} \\
\frac{\pi}{Z}\end{array}$} & 1 & 1 & $70.3 \%$ & 11 & 1.00 & $69.4 \%$ & $68.9 \%$ & $69.8 \%$ & 0.648 \\
\hline & 1 & 5 & $70.3 \%$ & 11 & 1.00 & $69.4 \%$ & $69.0 \%$ & $69.8 \%$ & 0.649 \\
\hline & 1 & 10 & $70.3 \%$ & 11 & 1.00 & $69.5 \%$ & $69.1 \%$ & $70.0 \%$ & 0.650 \\
\hline & 1 & 15 & $70.3 \%$ & 11 & 1.00 & $69.4 \%$ & $68.9 \%$ & $69.8 \%$ & 0.648 \\
\hline & 1 & 20 & $70.3 \%$ & 11 & 1.00 & $69.3 \%$ & $68.9 \%$ & $69.8 \%$ & 0.648 \\
\hline & 2 & 1 & $82.5 \%$ & 21 & 0.91 & $70.2 \%$ & $69.7 \%$ & $70.6 \%$ & 0.685 \\
\hline & 2 & 5 & $80.3 \%$ & 13 & 0.93 & $70.8 \%$ & $70.4 \%$ & $71.2 \%$ & 1.026 \\
\hline & 2 & 10 & $78.7 \%$ & 12 & 0.92 & $71.0 \%$ & $70.6 \%$ & $71.4 \%$ & 1.022 \\
\hline & 2 & 15 & $76.4 \%$ & 11 & 0.92 & $71.1 \%$ & $70.7 \%$ & $71.5 \%$ & 0.973 \\
\hline & 2 & 20 & $76.4 \%$ & 11 & 0.92 & $71.1 \%$ & $70.6 \%$ & $71.5 \%$ & 0.973 \\
\hline & 3 & 1 & $86.2 \%$ & 26 & 0.87 & $69.7 \%$ & $69.3 \%$ & $70.1 \%$ & 0.610 \\
\hline & 3 & 5 & $83.3 \%$ & 16 & 0.94 & $70.1 \%$ & $69.7 \%$ & $70.5 \%$ & 0.962 \\
\hline & 3 & 10 & $80.3 \%$ & 13 & 1.00 & $70.7 \%$ & $70.3 \%$ & $71.1 \%$ & 1.105 \\
\hline & 3 & 15 & $78.3 \%$ & 12 & 1.00 & $70.5 \%$ & $70.1 \%$ & $70.9 \%$ & 1.077 \\
\hline & 3 & 20 & $78.3 \%$ & 12 & 1.00 & $70.5 \%$ & $70.1 \%$ & $70.9 \%$ & 1.077 \\
\hline & 4 & 1 & $88.2 \%$ & 33 & 0.79 & $69.6 \%$ & $69.2 \%$ & $70.0 \%$ & 0.468 \\
\hline & 4 & 5 & $85.0 \%$ & 18 & 0.90 & $70.6 \%$ & $70.2 \%$ & $71.0 \%$ & 0.883 \\
\hline & 4 & 10 & $82.5 \%$ & 14 & 0.93 & $70.3 \%$ & $69.9 \%$ & $70.8 \%$ & 1.055 \\
\hline & 4 & 15 & $77.7 \%$ & 11 & 0.79 & $71.4 \%$ & $71.0 \%$ & $71.8 \%$ & 0.900 \\
\hline & 4 & 20 & $77.7 \%$ & 11 & 0.79 & $71.3 \%$ & $70.9 \%$ & $71.7 \%$ & 0.900 \\
\hline & 5 & 1 & $89.1 \%$ & 37 & 0.61 & $69.4 \%$ & $69.0 \%$ & $69.8 \%$ & 0.331 \\
\hline & 5 & 5 & $84.0 \%$ & 15 & 0.54 & $72.1 \%$ & $71.7 \%$ & $72.5 \%$ & 0.618 \\
\hline & 5 & 10 & $78.9 \%$ & 10 & 0.63 & $72.6 \%$ & $72.1 \%$ & $73.0 \%$ & 0.857 \\
\hline & 5 & 15 & $77.1 \%$ & 8 & 0.57 & $73.2 \%$ & $72.8 \%$ & $73.6 \%$ & 0.897 \\
\hline & 5 & 20 & $77.1 \%$ & 8 & 0.57 & $73.1 \%$ & $72.7 \%$ & $73.5 \%$ & 0.895 \\
\hline
\end{tabular}


Table 2.8. NESRS class schema and class proportions (Class Prop.) for an optimal scaling solution of a 3-part class-label precision with a landscape representativeness of $10 \%$. irMn = mean information retention; irSD $=$ standard deviation of information retention.

\begin{tabular}{lccc}
\hline Class Name - 33\% Precision 10\% Representativeness & Class Prop. $(\%)$ & irMn $(\%)$ & irSD $(\%)$ \\
\hline 100 Graminoid Marsh - Broadleaved Emergent * & 2.1 & 56.4 & 16.1 \\
\hline 100 Graminoid Marsh Cladium * & 8.5 & 89.8 & 7.6 \\
\hline $\begin{array}{l}\text { 67 Graminoid Marsh Cladium } \\
\text { 33 Graminoid Marsh Cladium Dense }\end{array}$ & 12.1 & 76.1 & 14.8 \\
\hline $\begin{array}{l}\text { 67 Graminoid Marsh Cladium } \\
\text { 33 Graminoid Marsh Cladium Sparse }\end{array}$ & 14.9 & 82.8 & 12.9 \\
\hline 100 Graminoid Marsh Cladium Dense * & 9.6 & 74.5 & 16.6 \\
\hline 100 Graminoid Marsh Cladium Sparse * & 18.0 & 90.3 & 8.1 \\
\hline 67 Graminoid Marsh Cladium Sparse & 20.7 & 54.8 & 16.3 \\
\hline 33 Graminoid Marsh Cladium & 1.1 & 70.8 & 17.6 \\
\hline 100 Graminoid Marsh Dense * & 5.5 & 60.1 & 17.7 \\
\hline 100 Graminoid Marsh Sparse * & 2.0 & 74.1 & 19.9 \\
\hline 100 Graminoid Marsh Typha * & 1.9 & 74.1 & 19.9 \\
\hline 100 Shrub Bayhead * & 3.3 & 74.1 & 20.2 \\
\hline 100 Shrub Salix * & 0.3 & 82.5 & 19.6 \\
\hline 100 Tree Bayhead *
\end{tabular}




\section{FIGURES}

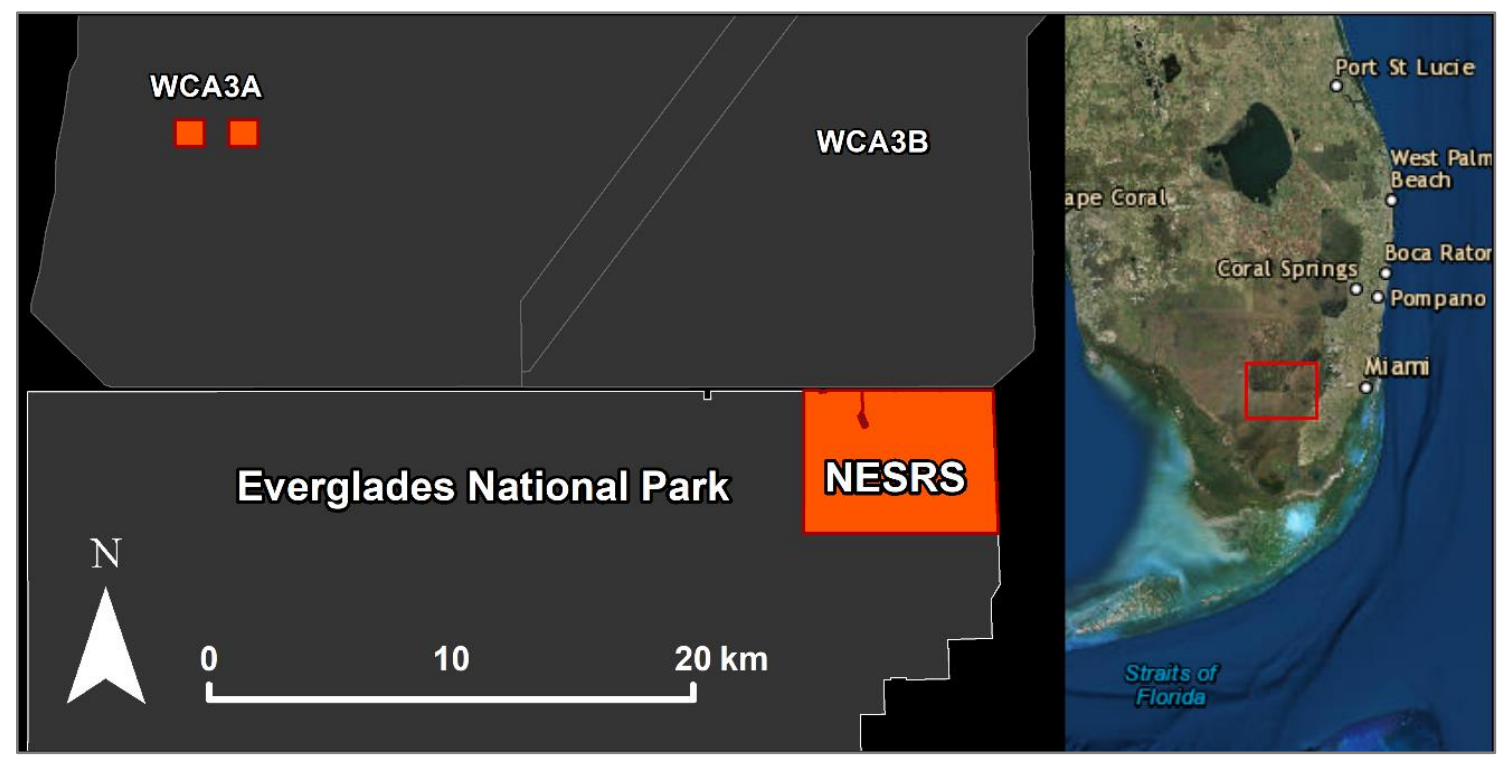

Figure 2.1. Study areas in Water Conservation Area 3A (WCA3A) and Northeast Shark River Slough (NESRS) within the boundaries of Everglades National Park. 


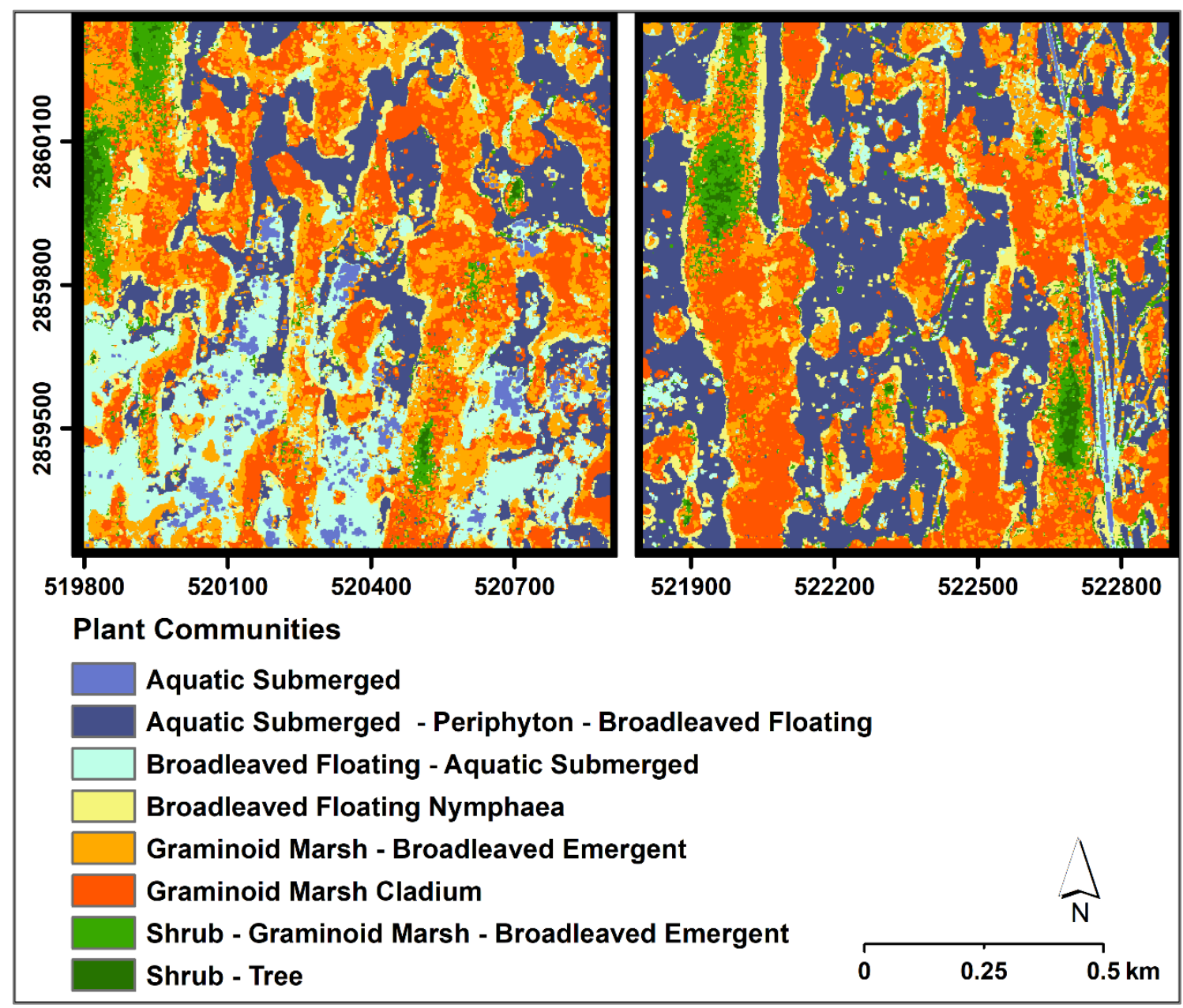

Figure 2.2. High-resolution plant communities for WCA3A. 


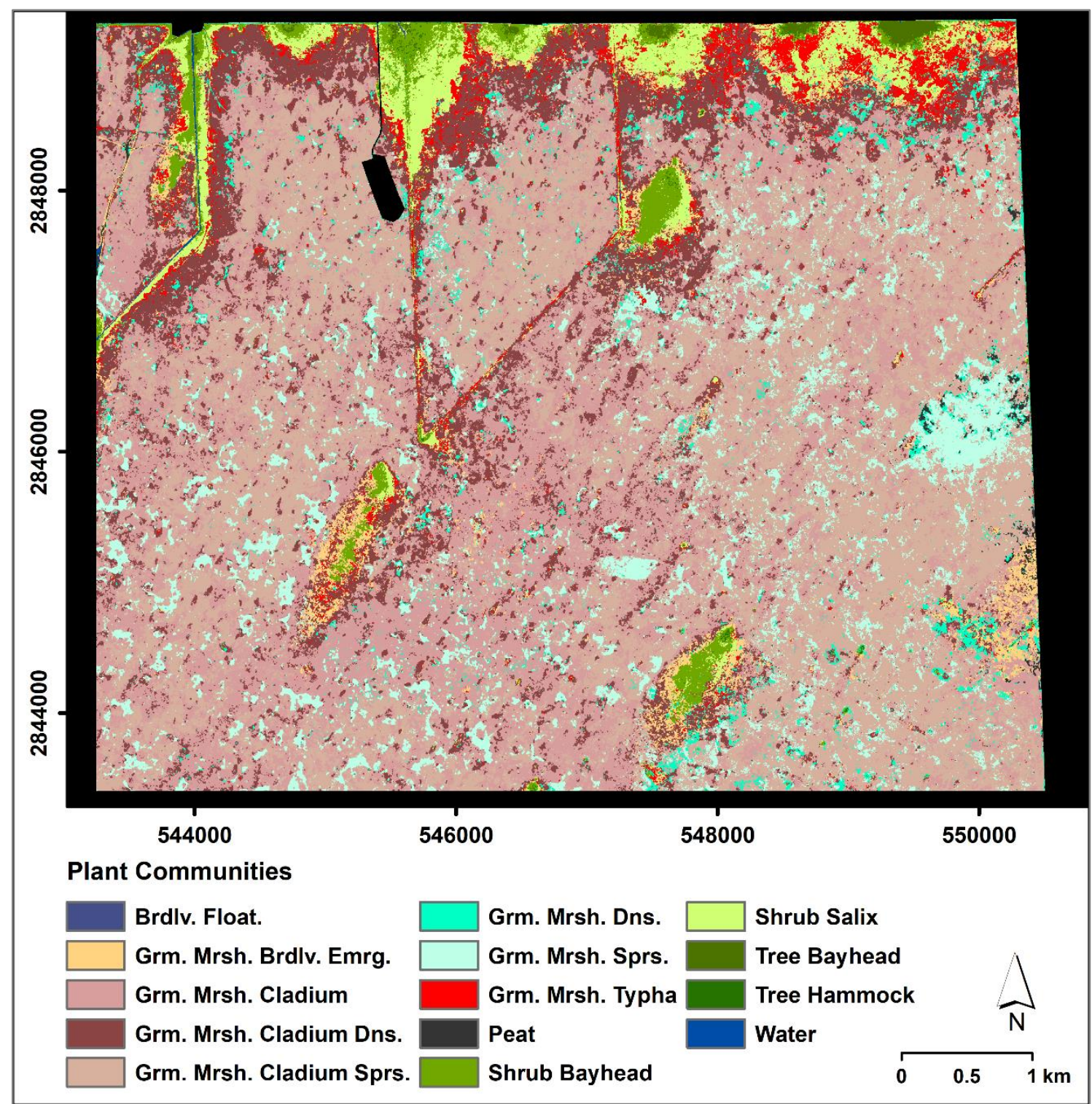

Figure 2.3. High-resolution plant communities for NESRS. Brdlv. = Broadleaved; Float. = Floating; Emrg. = Emergent; Grm. = Graminoid, Mrsh. = Marsh; Dns. = Dense; Sprs. = Sparse. 


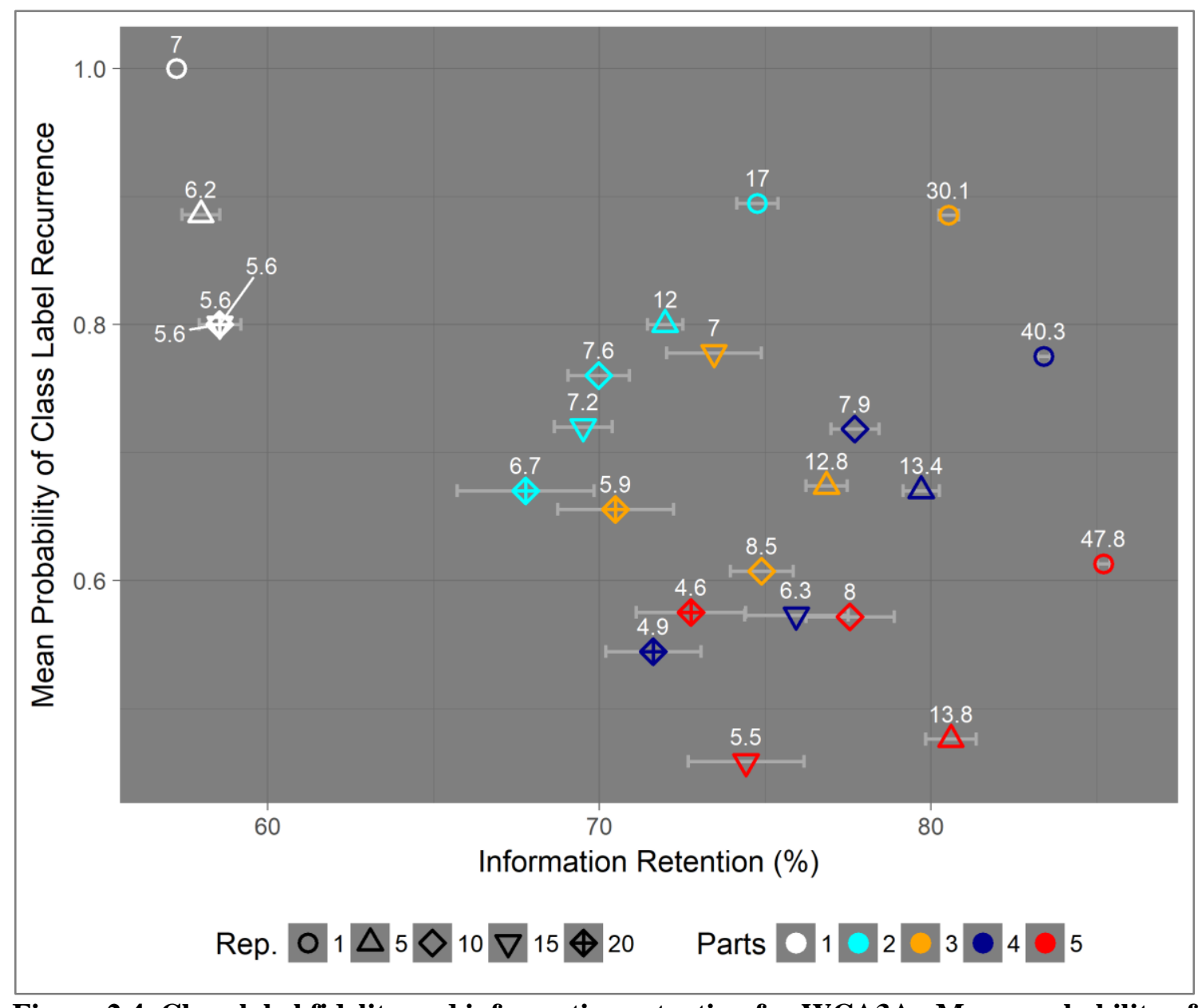

Figure 2.4. Class-label fidelity and information retention for WCA3A. Mean probability of class-label recurrence across all random-origin scaling results vs. mean landscape-level information retention. Models are displayed by class-label precision (Parts) in color and scaled landscape representativeness threshold (Rep.) with shape. Labels represent the mean number of classes generated for each model across the 10 random origins. 


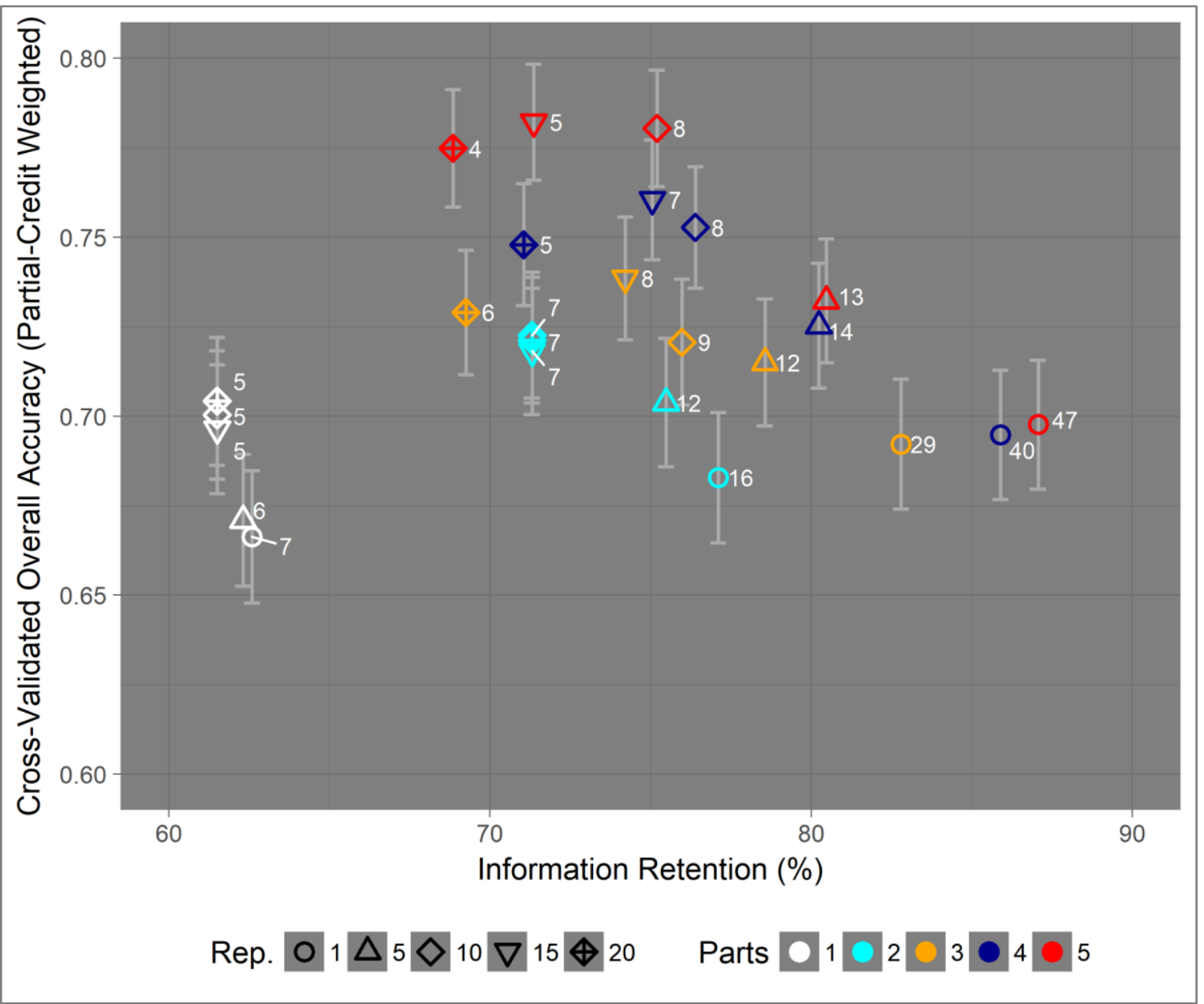

Figure 2.5. Cross-validated overall accuracy for WCA3A. For the same class-label precision (Parts), as representativeness (Rep.) increases and small classes are removed, information retention decreases and classification accuracy increases. Models are displayed by classlabel precision (Parts) in color and scaled landscape representativeness threshold (Rep.) with shape. 


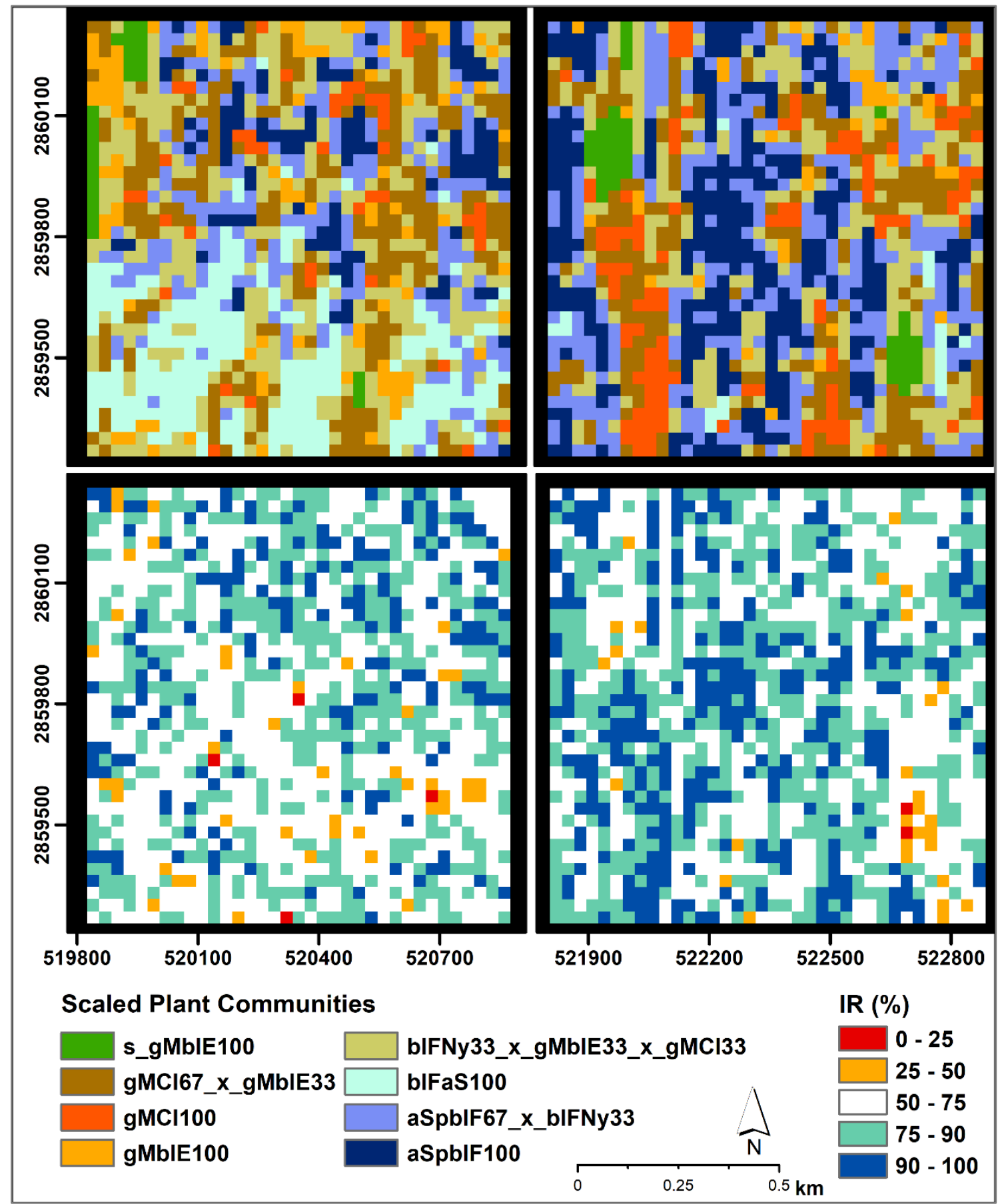

Figure 2.6. Scaled plant communities for WCA3A (top) and location-specific information retention (IR) in percent for the assigned community class label when compared to the highresolution map (Fig. 2) (bottom). Class label abbreviations: $s=$ shrub, gMblE = Graminoid Marsh - Broadleaved Emergent; gMCl = Graminoid Marsh Cladium; blFaS = Broadleaved Floating - Aquatic Submerged; blFNy = Broadleaved Floating Nymphaea $;$ aSpblF = Aquatic Submerged - Periphyton - Broadleaved Floating. 


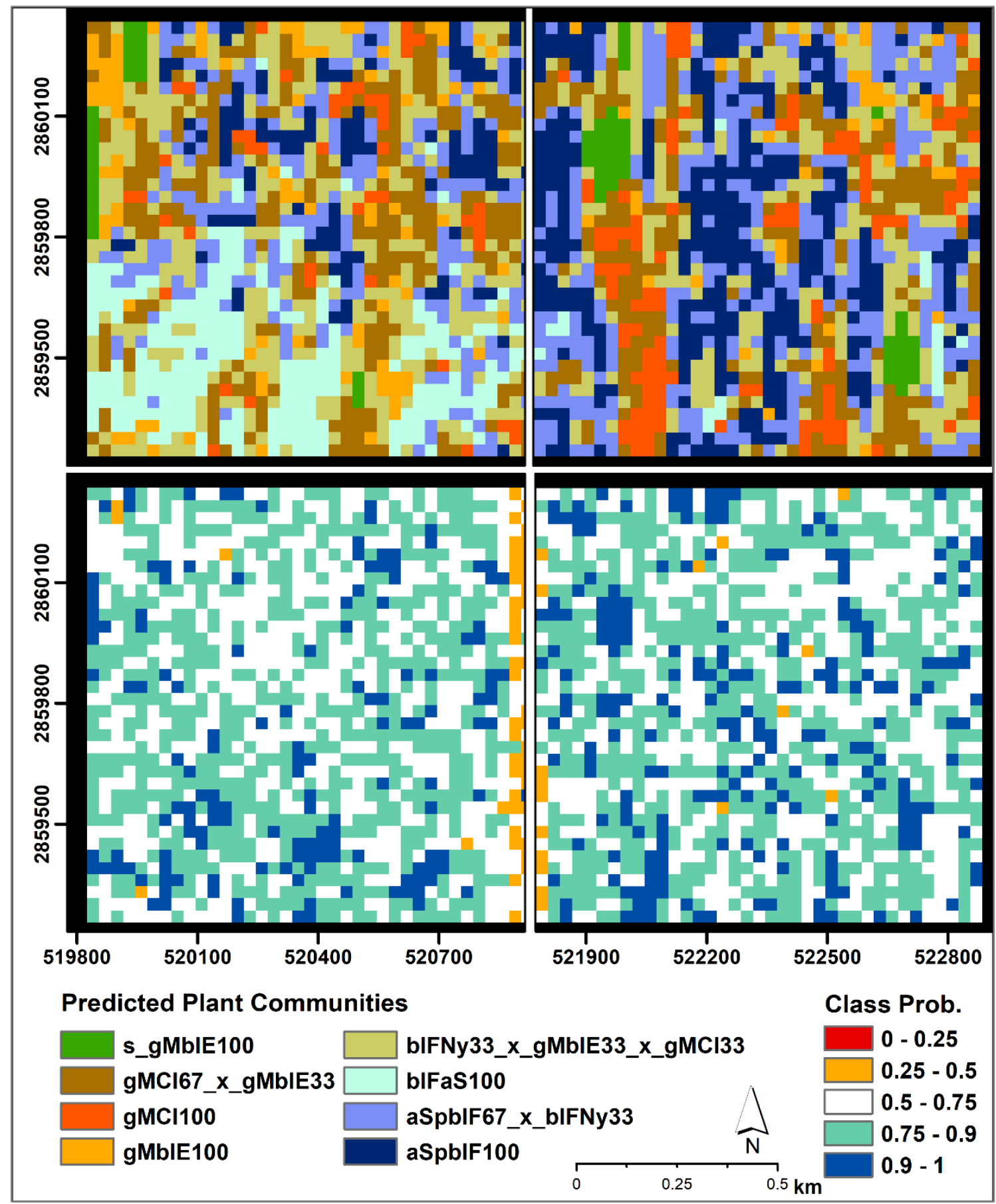

Figure 2.7. Scaled plant-community classes predicted from Landsat spectral data (top) and location-specific classifier probability for class-label assignment (bottom) for WCA3A. Class label abbreviations: $\mathrm{s}=$ shrub, gMblE $=$ Graminoid Marsh - Broadleaved Emergent; $\mathbf{g M C l}=$ Graminoid Marsh Cladium; blFaS = Broadleaved Floating - Aquatic Submerged; blFNy = Broadleaved Floating Nymphaea; aSpblF = Aquatic Submerged - Periphyton - Broadleaved Floating. 


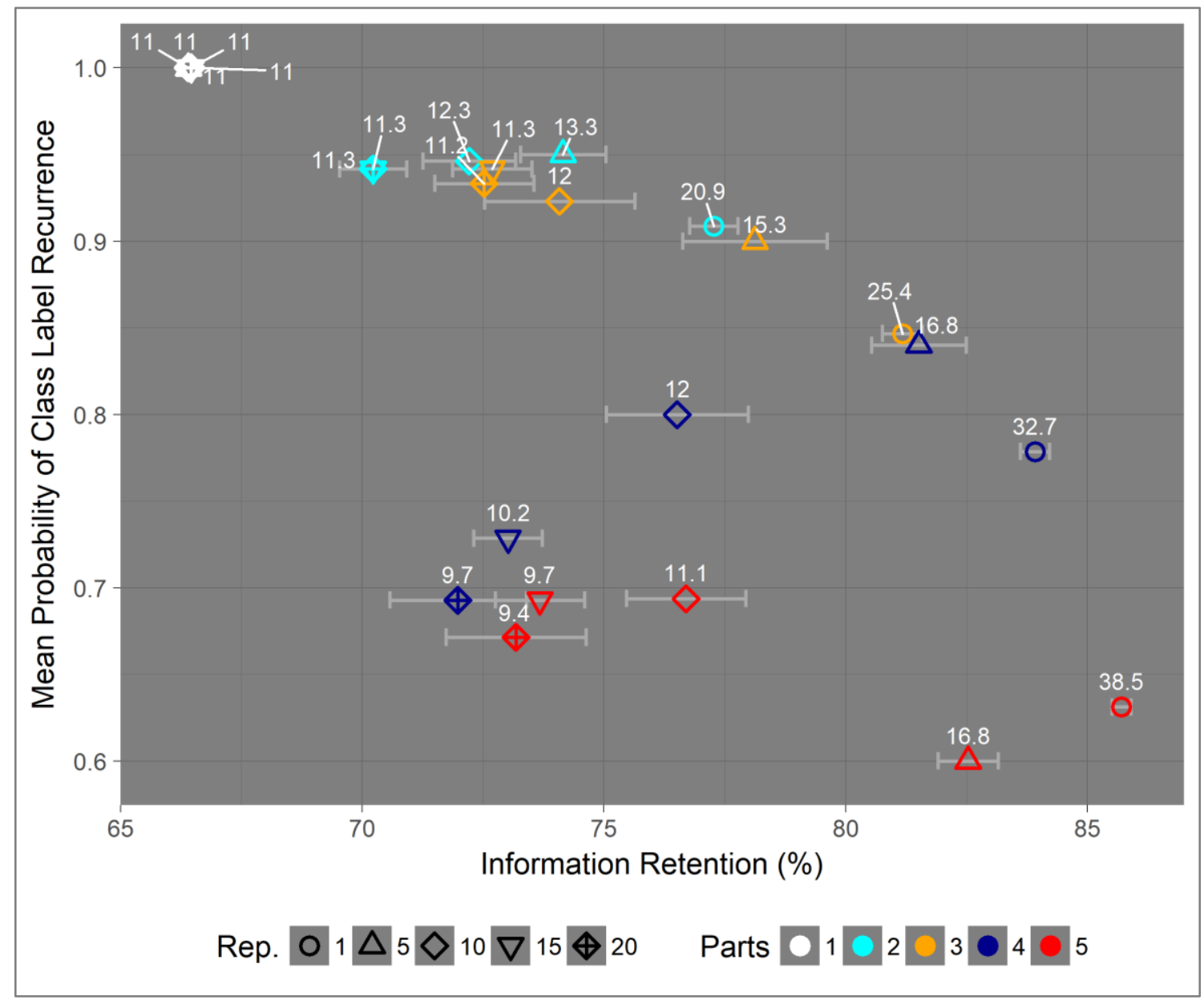

Figure 2.8. Class-label fidelity and information retention for NESRS. Mean probability of class-label recurrence across all random-origin scaling results vs. mean landscape-level information retention. Models are displayed by class-label precision (Parts) in color and scaled landscape representativeness threshold (Rep.) with shape. Labels represent the mean number of classes generated for each model across the 10 random origins. 


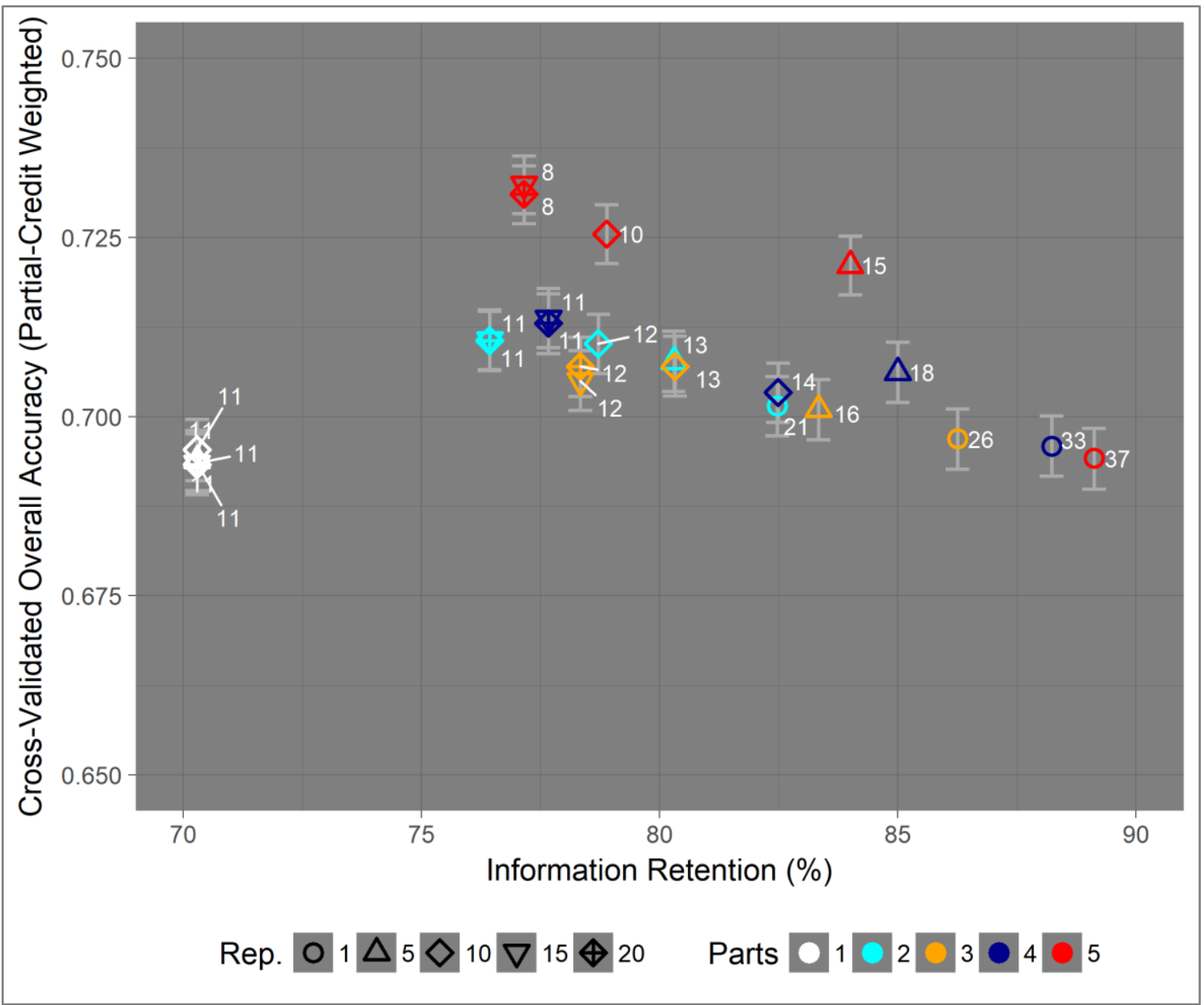

Figure 2.9. Cross-validated overall accuracy for NESRS. For the same class-label precision (Parts), as representativeness (Rep.) increases and small classes are removed, information retention decreases and classification accuracy increases. Models are displayed by classlabel precision (Parts) in color and scaled landscape representativeness threshold (Rep.) with shape. 


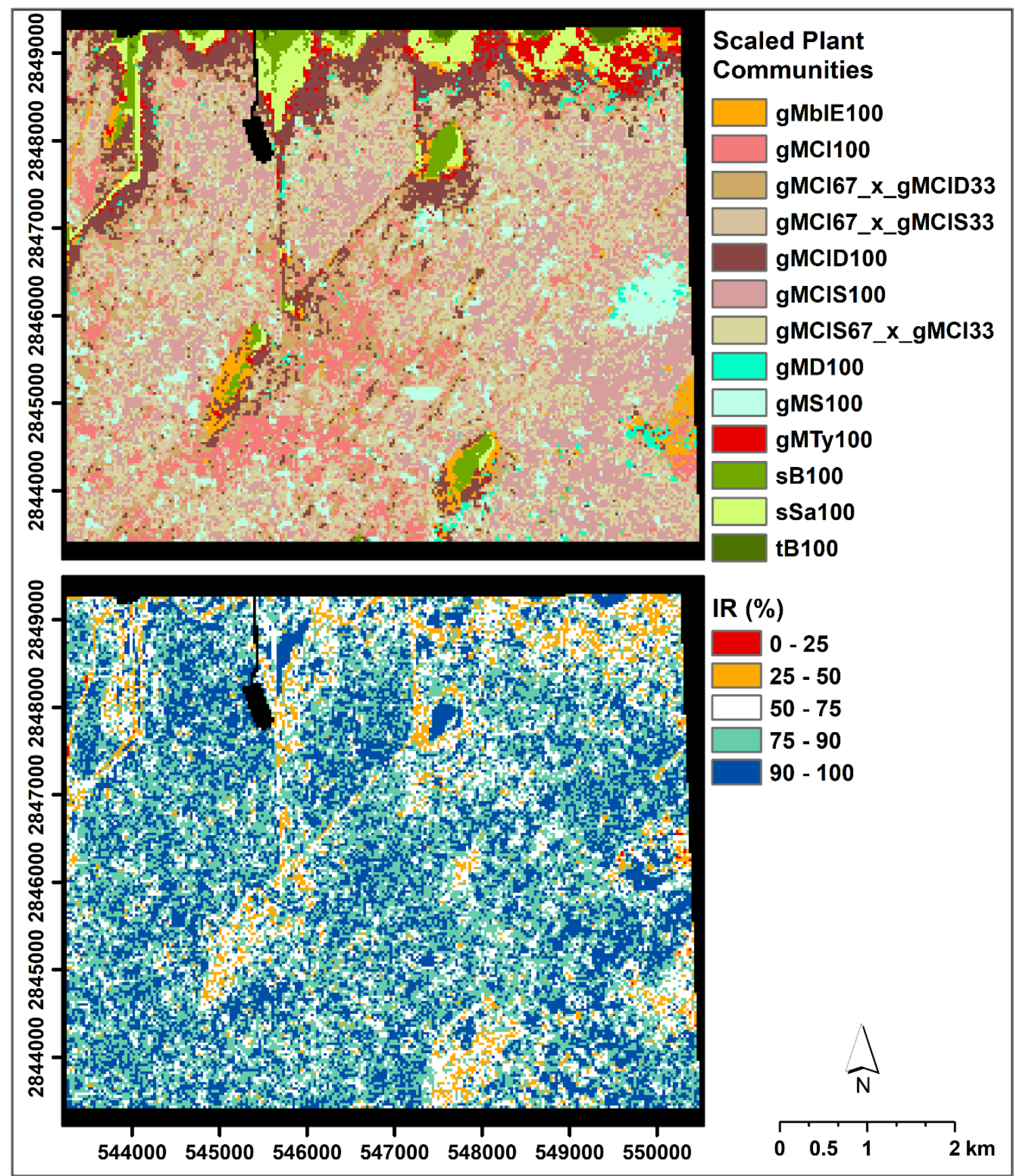

Figure 2.10. Scaled plant communities for NESRS (top) and location-specific information retention (IR) in percent for the assigned community class label when compared to the highresolution map (Fig. 3) (bottom). Class label abbreviations: gMblE = Graminoid Marsh Broadleaved Emergent; gMCl = Graminoid Marsh Cladium; gMCID = Graminoid Marsh Cladium Dense; gMCIS = Graminoid Marsh Cladium Sparse; gMD = Graminoid Marsh Dense; $\mathbf{g M S}$ = Graminoid Marsh Sparse; $\mathbf{s B}=$ shrub Bayhead; $\mathbf{s S a}=$ shrub Salix; $\mathbf{t B}=$ tree Bayhead. 


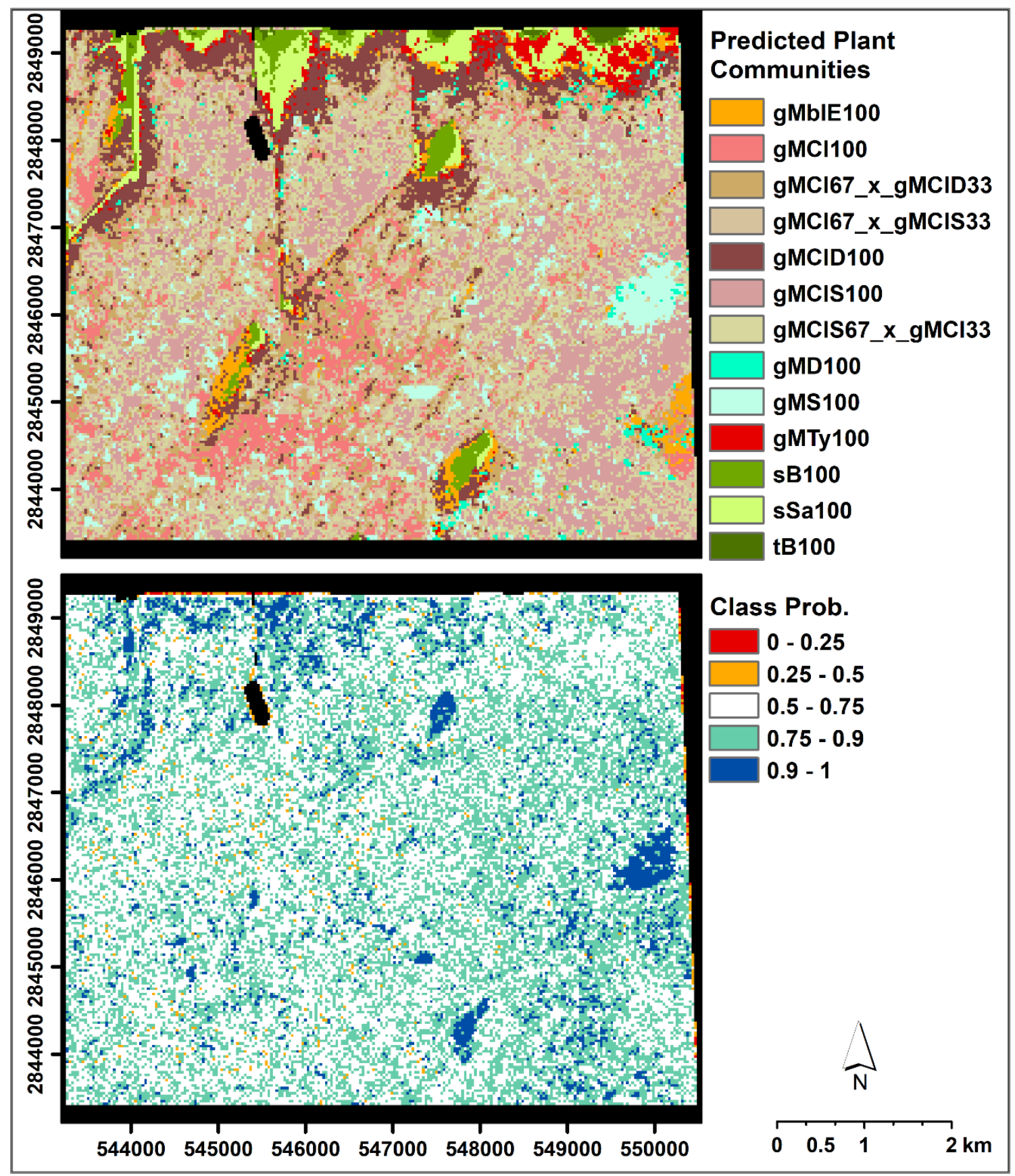

Figure 2.11. Scaled plant-community classes predicted from Landsat spectral data (top) and location-specific classifier probability for class-label assignment (bottom) for NESRS. Class label abbreviations: $\mathbf{g M b l E}$ = Graminoid Marsh - Broadleaved Emergent; $\mathbf{g M C l}=$ Graminoid Marsh Cladium; gMCID = Graminoid Marsh Cladium Dense; gMCIS = Graminoid Marsh Cladium Sparse; gMD = Graminoid Marsh Dense; gMS = Graminoid Marsh Sparse; $\mathbf{s B}=$ shrub Bayhead; $\mathbf{s S a}=$ shrub Salix; $\mathbf{t B}=$ tree Bayhead. 


\section{REFERENCES}

Bivand, R., T. Keitt, and B. Rowlingson. 2013. "Rgdal: Bindings for the Geospatial Data Abstraction Library". R package version 1.3. http://cran.rproject.org/package $=$ rgdal.

Breiman, L. 1984. Classification and Regression Trees. The Wadsworth Statistics/Probability Series. Belmont, CA: Wadsworth International Group.

Cohen, Jacob. 1968. "Weighted Kappa: Nominal Scale Agreement Provision for Scaled Disagreement or Partial Credit." Psychological Bulletin 70 (4):213-20. https://doi.org/10.1037/h0026256. https://doi.org/10.1037/h0026256.

DeAngelis, D. L., and S. Yurek. 2017. "Spatially Explicit Modeling in Ecology: A Review." Ecosystems 20 (2):284-300. https://doi.org/10.1007/s10021-016-0066-z.

Ganguly, S., R. R. Nemani, G. Zhang, H. Hashimoto, C. Milesi, A. Michaelis, W. Wang, et al. 2012. "Generating Global Leaf Area Index from Landsat: Algorithm Formulation and Demonstration." Remote Sensing of Environment 122:185-202. https://doi.org/10.1016/j.rse.2011.10.032.

Gann, D., J. H. Richards, and J. Sadle. 2015. "Vegetation Trends in Indicator Regions of Everglades National Park; Part II - Establishing Vegetation Trends in Northeast Shark River Slough - Everglades National Park Using WorldView-2 and Landsat Thematic Mapper Remotely Sensed Data". Florida City, FL. http://digitalcommons.fiu.edu/gis/29/.

Hijmans, R. J., and J. van Etten. 2010. "Raster: Geographic Analysis and Modeling with Raster Data". R Package Version 2.6. https://cran.rproject.org/web/packages/raster/index.html.

Jacob, F., and M. Weiss. 2014. "Mapping Biophysical Variables From Solar and Thermal Infrared Remote Sensing: Focus on Agricultural Landscapes With Spatial Heterogeneity." IEEE Geoscience and Remote Sensing Letters 11 (10):1844-48. https://doi.org/10.1109/LGRS.2014.2313592.

Knyazikhin, Y., J. Glassy, J. L. Privette, Y. Tian, A. Lotsch, Y. Zhang, Y. Wang, et al. 1999. "MODIS Leaf Area Index (LAI) and Fraction of Photosynthetically Active Radiation Absorbed by Vegetation (FPAR) Product (MOD15) Algorithm Theoretical Basis Document". http://eospso.gsfc.nasa.gov/atbd/modistables.html.

Kuhn, M., Contributins from J. Wing, S. Weston, A. Williams, C. Keefer, A. Engelhardt, T. Cooper, et al. 2016. "Caret: Classification and Regression Training”. R package Version 6.0. https://cran.r-project.org/web/packages/caret/index.html. 
Larsen, L., N. Aumen, C. Bernhardt, V. Engel, T. Givnish, S. Hagerthey, J. Harvey, et al. 2011. "Recent and Historic Drivers of Landscape Change in the Everglades Ridge, Slough, and Tree Island Mosaic." Critical Reviews in Environmental Science and Technology 41:sup1, 344-81. https://doi.org/10.1080/10643389.2010.531219.

Le Maire, G., C. Marsden, Y. Nouvellon, J. L. Stape, and F. J. Ponzoni. 2012. "Calibration of a Species-Specific Spectral Vegetation Index for Leaf Area Index (LAI) Monitoring: Example with MODIS Reflectance Time-Series on Eucalyptus Plantations." Remote Sensing 4 (12):3766-80. https://doi.org/10.3390/rs4123766.

Liu, Y., T. Hiyama, and Y. Yamaguchi. 2006. "Scaling of Land Surface Temperature Using Satellite Data: A Case Examination on ASTER and MODIS Products over a Heterogeneous Terrain Area." Remote Sensing of Environment 105 (2):115-28. https://doi.org/10.1016/j.rse.2006.06.012.

Lotsch, A., Y. Tian, M. A. Friedl, and R. B. Myneni. 2003. "Land Cover Mapping in Support of LAI and FPAR Retrievals from EOS-MODIS and MISR: Classification Methods and Sensitivities to Errors." International Journal of Remote Sensing 24 (10):1997-2016. https://doi.org/10.1080/01431160210154858.

Lu, D. 2006. "The Potential and Challenge of Remote Sensing-based Biomass Estimation.” International Journal of Remote Sensing 27 (7) 1297-1328. https://doi.org/10.1080/01431160500486732.

McVoy, C. W., W. P. Said, J. Obeysekera, J. A. VanArman, and T. W. Dreschel. 2011. Landscapes and Hydrology of the Predrainage Everglades. Gainesville, FL: University Press of Florida.

Myneni, R., Y. Knyazikhin, and T. Park. 2015. "MOD15A2H MODIS/Terra Leaf Area Index/FPAR 8-Day L4 Global 500m SIN Grid V006." NASA EOSDIS Land Processes DAAC. https://doi.org/doi: 10.5067/MODIS/MOD15A2H.006.

R Core Team. 2013. "R: A Language and Environment for Statistical Computing”. R Foundation for Statistical Computing. Vienna, Austria.

Rossiter, D. G. 2014. Technical Note: Statistical Methods for Accuracy Assesment of Classified Thematic Maps. Enschede, Netherlands. http://www.itc.nl/personal/rossiter/teach/R/R_ac.pdf.

Steltzer, H., and J. M. Welker. 2006. "Modeling the Effect of Photosynthetic Vegetation Properties on the NDVI-LAI Relationship." Ecology 87 (11):2765-72. https://doi.org/10.1890/0012-9658(2006)87[2765:MTEOPV]2.0.CO;2. 
Tian, Y., Y. Wang, Y. Zhang, Y. Knyazikhin, J. Bogaert, and R. B. Mynemi. 2002. "Radiative Transfer Based Scaling of LAI Retrieval from Reflectance Data of Different Resolutions." Remote Sensing of the Environment 84:143-59. https://doi.org/10.1016/S0034-4257(02)00102-5.

Van Den Boogaart, K. G., and R. Tolosana-Delgado. 2008. “compositions': A Unified R Package to Analyze Compositional Data." Computers and Geosciences 34 (4):320-38. https://doi.org/10.1016/j.cageo.2006.11.017.

Zhao, J., Y. Wang, H. Zhang, Z. Zhang, X. Guo, S. Yu, and W. Du. 2016. "Spatially and Temporally Continuous LAI Datasets Based on the Mixed Pixel Decomposition Method." SpringerPlus 5:516. https://doi.org/10.1186/s40064-016-2166-9.

Zweig, C. L., M. A. Burgess, H. F. Percival, and W. M. Kitchens. 2015. "Use of Unmanned Aircraft Systems to Delineate Fine-Scale Wetland Vegetation Communities." Wetlands 35 (2):303-9. https://doi.org/10.1007/s13157-014-06124. 
CHAPTER III

EFFECTS OF SAMPLING INTENSITY, SEASONALITY AND CLASSIFIER

SELECTION ON CLASSIFICATION ACCURACY, CONFIDENCE AND

EFFICIENCY IN WETLAND VEGETATION MAPPING

\section{INTRODUCTION}

Categorical maps are models of landscapes that have errors and uncertainty associated with them (Lunetta et al. 1991). Knowledge of class-specific and spatially explicit errors and uncertainties is crucial for evaluation of error and uncertainty propagation in spatially explicit models that incorporate such maps (Guisan and Zimmermann 2000; Heuvelink 2002; Kyriakidis and Dungan 2001; Langford et al. 2006; Wang et al. 2005;). The value of location-specific confidence estimation for quantitative estimates of geostatistical models has been demonstrated (Heuvelink 2002; Wang et al. 2005), but locationspecific uncertainty in the categorical information entering spatially explicit models is generally ignored, because it is not commonly available for categorical maps.

Furthermore, maximization of classification accuracy is a primary goal of classifier development, and comparison of performance between methods is a powerful tool to develop and fine-tune specific classifiers for an application of interest. However, to determine superiority of one classifier over another, comparing performance of two or more classifiers requires statistically sound proof of accuracy differences (Foody 2009). Commonly used metrics for map accuracy assessment in remote sensing are overall and class-specific map accuracies. Efficacy of a classifier is assessed by statistical analysis of the error matrix, calculating overall and class-specific accuracies and errors of omission and 
commission (Congalton and Green 1999); the error matrix is the cross-tabulation of random or stratified random samples (pixels or polygons) of a classified map vs. reference data consisting of class labels established from ground observation, aerial photography, or higher-resolution satellite images (Jensen 2015). Just comparing two overall accuracy values of two maps, however, cannot provide a solid base for determining which classifier achieved higher accuracies or if the differences were statistically significant. Comparing efficacy of two classifiers requires that accuracy estimates for two maps produced with different classifiers take into account sampling error and classifier consistency (Dietterich 1998; Langford et al. 2006; Smits, Dellepiane, and Schowengerdt 1999). Studies that compare classifiers often lack proof for a statistical difference of map accuracies between methods (Li et al. 2014; Mustapha, Lim, and Mat Jafri 2010; Szantoi et al. 2015), and studies that provide model-based accuracy with confidence estimates often use cross-validation methods with large overlapping sample sets in a re-sampling framework of non-randomlyselected training samples (Huang, Davis, and Townshend 2002; Rogan et al. 2008), which produces a less-biased classifier assessment, but not an unbiased estimate of map accuracy differences. In only a few cases has design-based statistical significance of classifier differences been established based on random samples (Pal 2005; Pal and Mather 2005).

Providing proof of superiority of one method over another is important if decisions on resource allocation for large mapping or monitoring programs is based on performance differences, and it is even more important if map production costs of the different evaluated methods vary significantly. In these cases, in addition to significant differences in accuracy, the increase in cost associated with an increase in accuracy needs to be considered in decision-making. 
The growing need for thematically accurate vegetation maps with high spatial resolution is supported by the trend in remote-sensor technology to deliver data with ever increasing spatial, temporal, spectral and radiometric resolutions. Increase in data resolutions, however, leads to an exponential per-area-unit increase in data storage and processing cost. Despite a drastic decrease in cost of powerful computing environments, production cost of maps with high accuracy and precision is an important factor when evaluating the efficacy of a classification method and the feasibility of its application to mapping projects or monitoring programs, especially when these programs require analysis of multi-temporal remotely sensed datasets for large spatial extents over long periods.

Optimizing classification methods (i.e., maximizing accuracy while reducing production costs) requires the evaluation of tradeoffs and interactions of process choices made when developing classification methods and classifiers. Three major decisions related to production cost and map accuracy for supervised classification methods are (1) the choice of a statistical classifier or algorithm, (2) the number of training samples required for adequate class separation, and (3) feature-space selection and dimensionality.

\section{Classifiers}

In remote sensing, maximum likelihood classifiers were the gold standard for a long time, but in the past $20+$ years the remote sensing community has increasingly embraced non-parametric classifiers and algorithms such as decision trees (Brown de Colstoun and Walthall 2006; Friedl and Brodley 1997), random forests (Belgiu and Drăguţ 2016; Breiman 2001; Gislason, Benediktsson, and Sveinsson 2006; Ho 1998), support vector machines (Cortes and Vapnik 1995; Boser, Guyon, and Vapnik 1992; Bovolo, Camps-Valls, and Bruzzone 2010; Foody and Mathur 2004), and neural networks (Atkinson and Tatnall 1997; 
Jakomulska and Radomski 2002; Miller, Kaminsky, and Rana 1995). Superiority of individual parametric or non-parametric classifiers or algorithms applied in remote sensing studies is not consistent across domains because of variability and interactions of classification schemes, sensor and data characteristics, and the variability in environmental conditions and class phenologies across studies; thus the optimal choice of classifier needs to be evaluated in the context of study-specific circumstances (Huang, Davis, and Townshend 2002; Li et al. 2014; Ozesmi and Bauer 2002). Ultimately, it is the multivariate variable space of class signatures that determines which classification algorithm is most efficient and accurate when compared to others under a specific set of circumstances.

In the case of multimodal or skewed distributions of spectral reflectance patterns in multivariate space, non-parametric classifiers are theoretically expected to be less biased than parametric classifiers, because, by definition, assumptions of the parametric classifiers are violated. However, machine learning algorithms are computationally more expensive than parametric statistical classifiers (e.g., maximum likelihood or naïve Bayes classifiers) when establishing a classifier from training sample data.

\section{Training Sample Size and Feature-Space Dimensionality}

The number of training samples required to construct an effective parametric classifier is estimated to be between 10 and 30 times the number of features in a dataset (Jensen 2005). Van Niel et al. (2005) suggest that the number of required training samples depends on data-, site- and phenomenon-specific characteristics of the study, and that casespecific evaluation of accuracy increase as a function of training sample size is required to determine the optimal sample size for classifier training (Foody, McCulloch, and Yates 1995; Shao and Lunetta 2012). As training sample size increases, calculation of class- 
specific multivariate mean and co-variance parameter estimates is computationally less expensive than multi-pass algorithms that search the dataset for optimal class boundaries or splits. While an increase in training samples adds cost in reference data gathering, digitization, and time required to train a classifier, it does not increase classification time once the classifier is established and applied to the full dataset.

With increasing sample size, processing time of classifier establishment is expected to increase exponentially, while accuracy is expected to increase according to the law of diminishing returns (Mitscherlich 1909). Hence, knowing the percent increase in processing time and accuracies as the number of training samples per class increases is more valuable than calculating the minimum number of samples required for adequate classifier performance. Even more useful is knowing the point at which adding samples does not significantly enhance classifier efficacy but keeps adding cost to the classification process. Estimating the maximum achievable accuracy, given a specific feature set, provides valuable information in the context of budget constraints.

Accuracy and efficiency also varies with feature-space dimensionality (e.g., uni- vs. multi-temporal feature sets). Increasing the feature space increases both the time required for classifier establishment and the classification time needed to assign a class to each sample (pixel). Feature space can be expanded using multi-seasonal images and/or texture variables and indices derived from the reflectance bands of an image. In many cases, including texture variables has increased classification accuracy (Rodriguez-Galiano et al. 2012; Szantoi et al. 2015), but adding more sophisticated spatio-texture variables (Li et al. 2014) into the feature space increased processing time and data storage requirements. 
Using multi-seasonal data to capitalize on phenological differences among classes has also increased accuracy in differentiating and mapping tree species (Dymond, Mladenoff, and Radeloff 2002; Gao et al. 2015), tidal marsh vegetation (Gao and Zhang 2006; Gilmore et al. 2008), and agricultural crops (Sakamoto et al. 2005). Multi-seasonal data is expected to have greater information content than local texture if the phenotypic variability of classes and the associated spectral patterns that differentiate these classes are greater than the spatial variability of those spectral patterns at any given point in time. Including multi-seasonal data and local texture is justified if the class-specific accuracy is improved significantly.

However, such inclusion increases the number of features, and as the dimensionality of the feature space increases, the minimum number of required training samples increases as well (Jain and Chandrasekaran 1982; Kanal and Chandrasekaran 1971). Additional spectral reflectance patterns (i.e., multi-seasonal data) that capture phenological cycles of classes are expected to add greater information value to a classifier than adding training samples from a uni-seasonal dataset, and hence an increase in feature space could potentially allow for a reduction in the number of training samples while maintaining or even increasing map accuracy. It is, therefore, of interest to evaluate the effects and interactions of feature set expansion, training sample size, and classification accuracy. Understanding these interactions and trade-offs and their effects on class- and location-specific classifier accuracy and cost will allow optimization of case-specific classification methods.

The above discussion has described current limitations and constraints when choosing a classifier for mapping using remotely sensed data: (1) the limitations of previous studies in unbiased evaluation of performance differences of supervised classification 
methods; (2) the lack of spatially exhaustive, location-specific, confidence estimates of maps; and (3) the need to make informed decisions about resource allocation. Given this, the objectives of this paper were to develop a framework that facilitates the evaluation of classification performance as a function of three major aspects of the classification process: (1) choice of classification algorithms; (2) the sizes of training sample sets; and (3) data volume and the number of variables used in the feature space.

A framework to model the interactions of training sample intensity, feature space, and their effects on mapping accuracies and processing time was developed. This framework was then used to develop and optimize a mapping method for wetland plant communities from high-resolution, multi-spectral, satellite data. The Everglades in southern Florida, USA, was selected as a study site because large scale restoration efforts are being implemented in this wetland (National Research Council 2014; Sklar et al. 2001), and remote sensing technology can provide an effective way to monitor and evaluate restoration success. Estimating monitoring cost and expected accuracies for alternative monitoring strategies is crucial for adequate budget allocation.

Challenges wetlands present to using spectral remote sensing from single images are seasonally varying water levels and phenology of vegetation in response to water cycles and climatic conditions (Gann et al. 2015; Jones, Desmond, and Henkle 2012; Ozesmi and Bauer 2002). Fluctuating water levels lead to alternate submersion and exposure of vegetation and substrate. Hence, depending on occurrence along a topographic gradient, a plant community type is expected to manifest a variety of spectral signatures. In addition, seasonal occurrence of algae and cyanobacteria in the form of epiphytic periphyton attached to floating or emergent vegetation or of benthic periphyton mats adds to inter-seasonal and 
inter-annual spectral variability for many vegetation communities (Gann et al. 2015; Jones 2011; Kim et al. 2014). This bi-seasonal phenological cycle, therefore, makes the Everglades wetland a good environment to explore the benefits and trade-offs of utilizing multi-seasonal datasets and to model classification performance that allows decision makers to develop a cost-effective monitoring strategy.

\section{METHODS}

Study Area

The study was conducted in two $1-\mathrm{km}^{2}$ plots located in the southwestern corner of Water Conservation Area 3A, part of the greater Florida Everglades ecosystem, USA (Fig. 3.1). This ridge and slough landscape is comprised of higher elevation ridges dominated by Cladium jamaicense (graminoid) interspersed with broadleaf species and island-forming shrub and tree species. The ridges alternate with lower elevation sloughs that are dominated by the floating-leaved species Nymphaea odorata, Utricularia spp. and submerged aquatic species (Fig. 3.1); shallower sloughs and transitional areas are dominated by dense Eleocharis spp. and other short sedges, grasses, and broad-leaved emergent species.

\section{Classification Scheme}

Two morphological landscape classes were differentiated, (1) graminoid- and broadleaf-dominated ridges, interspersed with isolated shrub and tree-dominated communities, and (2) slough communities including submerged aquatic vegetation, floating-leaved and emergent broadleaf vegetation, and periphyton (Tbl. 3.1). For each of the morphological classes, four lower-level plant community classes were established.

In ridges a monotypic Cladium-dominated community was differentiated from a graminoid-broadleaf mixed marsh class that primarily included mixes of the graminoid 
species (Eleocharis spp., and Panicum spp.), and emergent broadleaf species such as Peltandra virginica, Sagittaria lancifolia, Crinum americanum and Pontederia cordata. The shrub and tree component of the ridges was divided into a less-dense shrub community made up of Salix caroliniana, Myrica cerifera, Ilex cassine, Persea borbonia, and Chrysobalanus icaco, interspersed with several broadleaf and fern species, and a dense shrub-tree class that was dominated by dense shrubs and trees (Tbl. 3.1).

The morphological slough class was also divided into four community classes (Tbl. 3.1). A submerged aquatic class was identified as mainly open water with submerged or floating non-broadleaf vegetation (mainly Potamogeton illinoensis, Chara spp., and Utricularia spp.). This was differentiated from a submerged aquatic and mixed broadleaf class. A third class had the same mix but periphyton dominated. The fourth class was a Nymphaea odorata-dominated class. Short graminoid patches of Eleocharis, Rhynchospora or Panicum in sloughs have great inter-annual variability (Zweig and Kitchens 2009) and were not encountered densely enough during the time frame of the study to be acknowledged in our classification scheme. In most cases, these short graminoid taxa were present at low densities within Nymphaea slough and dominant submerged aquatic species. A complete list of classes is provided in Table 3.1.

\section{Datasets}

Multi-spectral bi-seasonal satellite data were used for the spectral separability analysis and detection of vegetation types. In situ sample data were gathered and used for confidence building in interpreting vegetation types from two sources of aerial photography, a very-high resolution $(3-5 \mathrm{~cm})$ aerial photography dataset acquired by an unmanned aerial 
system and stereo- and ortho-photography were used for training set establishment and accuracy assessment (Fig. 3.1).

\section{Multi-spectral Multi-temporal Satellite Data}

Two WorldView-2 (WV-2; Satellite Imaging Corp., Houston, TX) datasets with a spatial resolution of $2 \mathrm{~m}$ and 8 spectral bands ranging from $400 \mathrm{~nm}$ to $1,040 \mathrm{~nm}$ were acquired on May $5^{\text {th }}, 2011$, and October $20^{\text {th }}$, 2012. Images were geometrically coreferenced to 1-ft ortho-photographs of 2012 (2012 Digital Orthophotos - Miami-Dade County, Tallahassee, FL) and atmospherically corrected using ENVI's Fast Line-of-sight Atmospheric Analysis of Hypercubes (FLAASH) (Exelis Visual Information Solutions, Boulder, Colorado).

\section{Reference Data}

Training samples for each of the vegetation classes were collected in the field at the time of acquisition of the UAS aerial photography in September 2012 (Zweig et al. 2015). Primary reference information source for training set digitization and evaluation of random samples for accuracy assessment was the very-high resolution mosaic of the UAS aerial photography produced by the United States Army Corps of Engineers in collaboration with University of Florida (Zweig et al. 2015). The photographs were taken in September 2012, just one month before the WV-2 dataset for the wet season was acquired. The 10-megapixel Olympus® E-420 ${ }^{\mathrm{TM}}$ (Olympus Corp., Shinjuku, Tokyo, Japan) digital single-lens reflex (dSLR) camera with a fixed focal length $25 \mathrm{~mm}$ Olympus ${ }^{\circledR}$ Zuiko Digital ${ }^{\mathrm{TM}}$ pancake lens was mounted on a Nova 2.1 (Altavian Inc., Gainesville, FL) fixed-wing unmanned aerial system (UAS) developed by the University of Florida Unmanned Aircraft Systems Research Program. Two mosaics of approximately $1 \mathrm{~km}^{2}$ each were generated from the photographs 
using Agisoft LLC PhotoScan software (Agisoft LLC, St. Petersburg, Russia). The mosaics had a nominal spatial resolution of $5 \mathrm{~cm}$, with raw data images providing even higher spatial resolutions of $1-3 \mathrm{~cm}$.

In addition to the high-resolution aerial mosaic, a stereo-photography set from 2011 was used for height references to establish shrub and tree heights and to separate them from shorter woody and broadleaved vegetation. The stereo aerial photography was acquired with an UltraCam X (Vexcel Imaging GmbH., Graz, Austria) frame-based digital camera as part of the Comprehensive Everglades Restoration Plan (CERP) Restoration Coordination and Verification (RECOVER) vegetation mapping project.

Vegetation detection and interpretation from the high-resolution aerial photography was evaluated on the basis of two independent visual interpretations of $6492 \times 2 \mathrm{~m}$ grid cells interpreted by two field-trained interpreters. Class detection agreement for 19 species-level classes was $96.5 \% \pm 3.2 \%$ (Mean $\pm S D$ ). Classes that were most consistently detected with an agreement $\geq 98 \%$ by both interpreters were broadleaved species Nymphaea odorata, Sagittaria lancifolia, and Pontederia cordata, graminoid species Cladium jamaicense and Panicum hemitomon, and all tree species (Gann and Richards 2013).

\section{Training and Accuracy Evaluation Dataset}

Training samples for each class for the spectral separability analysis from WV-2 data were digitized from the high resolution UAS aerial photography and the stereo photography. To estimate sampling error for classification results on the basis of random samples with varying sample numbers, the pool of training samples had to be large. A minimum of 1,000 samples (pixels) per class were digitized, for a total of 17,000 pixels. 
For the design-based classifier accuracy evaluation a simple random sample of an additional 2,500 samples, or $0.5 \%$ of the population of pixels, was drawn within the study area (two $1-\mathrm{km}^{2}$ areas of 250,000 pixels of $4 \mathrm{~m}^{2}$ each). No random sample for accuracy assessment was a member of the training sample set. A wet and dry season community class was assigned to each training sample and each accuracy assessment sample on the basis of a combined evaluation of the $3 \mathrm{~cm}$ resolution aerial mosaic, stereo-photography and spectral signature of the two WV-2 images.

\section{Model Definitions and Evaluation Framework}

A full factorial sampling and evaluation framework was developed that facilitated evaluating the effects of classifier algorithm choice, feature selection, and training sample intensity on accuracy and efficiency. At the highest level of the evaluation framework, a wrapper method for feature selection was incorporated. The feature selection wrapper provided meaningful feature subspaces to compare classification accuracy differences for datasets of single and multi-seasonal reflectance data only and in combination with local texture variables. Feature subspaces of interest were the reflective bands of a single wet season ( 8 bands) vs. a single dry season ( 8 bands) vs. the combined wet and dry season feature space of both images (16 bands). For the dry and wet season images, texture or local spectral variability in the form of data ranges within a $3 \times 3$ kernel was calculated for each of the eight original reflective bands of each image. For each of the three datasets, a version with the local data range was included in the feature-space subsets, resulting in a total of six variable sets or feature subspaces (three with and three without texture) (Tbl. 3.2). Embedded, classifier-specific, feature selection procedures then searched for optimal feature subsets within those subspaces. The local texture bands (eight per image) were 
generated in R (R Core Team 2013) using the focal function in the "raster" package (Hijmans and van Etten 2010), resulting in a data cube with 32 layers (eight dry, eight wet, and their 16 texture layers).

Each of the six described variable sets was evaluated for training sample sizes of 12 , 25, 50, 100, 200, and 300 samples per class. For each of the six sample intensities, one parametric and one non-parametric classifier method was constructed and applied to the full dataset to generate classified maps and their spatially explicit, algorithm-determined class label probability maps. The full factorial design yielded 72 models of interest (Tbl. 3.2). To account for sampling error in the model performance comparison and to establish classifier stability, each model was evaluated 10 times using bootstrap re-samples of the training sample pool of 1,000 samples per class for each of the training sample intensities. Each of the 60 sample sets (10 per sampling intensity) was evaluated for each of the six variable sets and by two classifiers for a total of 720 models (60x6x2).

Random forest and naïve Bayes classifiers were selected to represent the nonparametric and parametric methods, respectively. Best practice tuning parameters for each of the classifiers were employed to ensure that model performance was optimized for each model individually. For the random forest models, test runs for different samples sizes and variable sets concluded that 200 trees were a good threshold beyond which no significant increase in classification accuracy was observed, and therefore the number of trees was set to a constant 200. The parameter for optimal number of random variables selected at each split ("mtry") was established for each random forest model with a built-in tuning routine, considering all possible options from two to the maximum number of all features in the evaluated feature subset (Kuhn et al. 2016The naïve Bayes tuning parameters were optimal 
feature selection using a backward feature selection algorithm and whether a kernel density or normal density estimator were used (Weihs et al. 2005). For both classifiers, the count of optimal features was recorded for each model.

\section{Classifier Evaluation}

Performance of all classifier models was evaluated on the basis of (1) overall accuracy; (2) computational efficiency of the classifier, including training time and prediction time; (3) location-specific class confidence; and (4) class-specific user's and producer's accuracies. Overall accuracy for each of the 720 models was estimated from confusion matrices that were constructed for the 2,500 random samples. Class labels that were assigned by the classifier were cross-tabulated with the analyst-assigned labels and accuracy calculated as a percentage of correctly classified samples of the 2,500 labeled random samples. Confidence intervals of accuracy for all 72 models were estimated from the results of the 10 random sample training sets evaluated for each model.

Computational efficiency of the classifiers was assessed for classifier training time and classifier prediction time. Training time was then modeled as a function of training sample size, the number of variables in the model-specific feature subspace, and the number of classes. Prediction time was modeled as a function of the optimal number of features selected from the feature subspace and the number of classes in the classification scheme. The number of optimal features could vary from 2 to the dimensionality of feature subspace of 8,16 or 32 variables, and the number of classes varied between 8 for the single season models and 17 wet/dry season class combinations for the bi-seasonal models. The 17 combined wet/dry season classes were composed of the eight original ridge and slough classes and nine slough classes that had different vegetation class labels assigned for the 
two seasons (e.g., submerged aquatic vegetation in the dry season that was dominated by periphyton or floating broadleaf species during the wet season).

Model performance in terms of overall accuracy and efficiency differences between models were evaluated using Mann-Whitney tests, pooling accuracy and timing results for the 10 replicates of each model $(\alpha=0.05)$. Differences were assessed for classifier type, feature subspace, and sampling intensity by estimating the shift in median locations between models and their 95\% confidence intervals (CI) using the Hodges-Lehmann estimator (Hodges and Lehmann 1963). Median location shift estimates of accuracy and efficiency were used to determine whether the differences, when statistically significant, were also meaningful in the context of mapping vegetation classes, thus justifying more complex classifiers and more training samples (Foody 2009).

The effects of increase in sampling intensities and feature subspace expansion on overall accuracy and processing time were evaluated by fitting regression models that aimed at estimating approximate increase in percent accuracy and processing times for percent increase in sampling intensity and feature space (Foody and Arora 1997). Increase in overall accuracy was expected to follow the law of diminishing returns as sampling intensity increased with an upper limit bound on accuracy. Asymptotes of accuracy as a function of sampling intensity for each variable set were estimated based on a nonlinear growth model (Equation 3.1).

$$
o a=\beta 1 * \alpha e^{(-\beta 2 * \operatorname{smpInt})}
$$

Equation 3.1

In equation $3.1 \mathrm{oa}$ is the overall accuracy, smpInt is the sampling intensity, and $\beta 1$ the predicted accuracy boundary or asymptote. Asymptote confidence intervals for overall accuracies for the different models were established using a bootstrap method with 999 re- 
samples (Baty et al. 2015). Results of the overall accuracy and computational efficiency analysis informed the selection of classifier type for which location-specific and classspecific assessments were performed.

Location-specific classification consistency as an indicator of confidence was evaluated in a spatially explicit and exhaustive manner. Location-specific confidence was defined as the proportion of the class most frequently assigned a pixel. Spatially explicit classification confidence was calculated from the classified maps of each model's 10 replicates. Significance of differences in location-specific confidence was tested with a onesided pairwise-paired Wilcoxon signed-rank test (Wilcoxon 1945) evaluated pairwise for each combination of sampling intensities within variable sets and between variable sets for equal sampling intensities. The datasets were paired by pixel. The null hypothesis was that pairs did not differ in confidence with sampling intensity and feature-space dimensionality, while the alternative hypothesis was that confidence increased with sampling intensity and feature-space dimensionality. Test $p$-values were adjusted using the Bonferroni correction for multiple comparisons by multiplying $p$-values by the number of pairwise comparisons (15 variable sets and 15 sampling intensity combinations). Location-specific confidence estimates were then aggregated across all pixels and the percent area of confidence of at least $90 \%$ was compared across all models.

Class-specific user's and producer's accuracies (Congalton and Green 1999) were assessed from the confusion matrices of the models for the sampling intensity beyond which increase in overall accuracy and location-specific confidence increase were not justified (significant). Significance in differences of class-specific user's and producer's accuracies was assessed for each class and for 7 selected combinations of variable sets (feature 
subspaces) using Mann-Whitney tests. Accuracy estimates for the 10 replicates of each model were pooled and shifts in median locations between models and their $95 \%$ confidence intervals $(C I)$ were estimated using the Hodges-Lehmann estimator (Hodges and Lehmann 1963). Class-specific accuracies were evaluated for each of the original eight classes (four slough and four ridge classes) as they occurred in the wet and dry seasons, because I was interested in the gain of individual class map accuracy of each class and not in the combined wet-dry class combination detection accuracies.

\section{Computing Environment}

All data analyses were performed with R (Revolution R 7.4, Revolution Analytics, Mountain View, CA). Classifications were performed in the unifying modeling framework of the "caret' package ( et al. 2016), using the "rf" function for random forest and the naïve Bayes algorithm as implemented in the "klaR" package (Weihs et al. 2005). In addition, packages "raster" (Hijmans and van Etten 2010), "rgdal" (Bivand, Keitt, and Rowlingson 2013), "foreach" and "doParallel" (Revolution Analytics and Weston 2013) were extensively used. All processing was performed on a desktop computer with six dedicated i7-4930K $3.4 \mathrm{GHz}$ processors and $32 \mathrm{~GB}$ of dedicated RAM with all read and write operations executed to and from a local static-state disc. No other processes or applications were allowed to run during the processing timeframe to ensure comparability of timing results.

\section{RESULTS}

\section{Overall Accuracy}

For each of the 72 model types, random forest classifier models consistently had significantly higher overall accuracies compared to the corresponding naïve Bayes models 
$(p<0.001 ; N=10)$ (Fig. 3.2). This was the case for all variable sets and sampling intensities except for 12 samples. For 12 samples and the "Dry + texture" variable set, the $3 \%$ higher accuracy of the random forest classifier was insignificant $(C I:-0.2 \%$ to $5.2 \%, p=0.064)$, and for wet and bi-seasonal datasets with and without texture, $p$-values were between 0.001 and 0.005 (Fig. 3.2, Tbl. 3.3). Significant differences in accuracy between the naïve Bayes and random forest classifier ranged from 5.5\% (CI: 3.2\% to 7.6\%) for "Dry" season models of 12 samples to $17.8 \%$ (CI: $16.2 \%$ to $19.0 \%$ ) for wet season data and 300 training samples (Fig. 3.2, Tbl. 3.3).

Overall accuracy for both classifiers was consistently higher for wet season than for dry season models, and differences were highest for bi-seasonal vs. dry season only data (Fig. 3.2, Tbl. 3.4 and 3.5). Mean increase in median accuracy across all sampling intensities for wet season over dry season data was $8.9 \%(S D= \pm 0.9 \%, p<0.001$, pairwise $t$-test) for naïve Bayes and $12.1 \%(S D= \pm 1.1 \%, p<0.001)$ for random forest (Tbl. 3.4 and 3.5). Combining data of both seasons increased accuracy on average by an additional $5.6 \%$ $(S D= \pm 0.42 \%, p<0.001)$ and $3.9 \%(S D= \pm 1 \%, p<0.001)$ for naïve Bayes and random forest, respectively (Tbl. 3.4 and 3.5). Adding texture had a significant positive effect only for dry season data and naïve Bayes models, increasing accuracies by $1 \%(S D= \pm 0.54 \%, p$ $=0.012)$. In the case of random forest models, the use of texture variables did not have a significant effect for bi-seasonal and wet season data, but decreased accuracy on average by $0.5 \%(S D= \pm 0.6 \%, p=0.048)$ for the dry season $($ Tbl. 3.4 and 3.5$)$.

For the naïve Bayes algorithm, asymptotic accuracy as modeled with a non-linear growth model ranged from $61.2 \%(C I: 60.7-61.7 \%$, bootstrapped $n=999)$ for dry season data to $77 \%(C I: 75.6-87.3 \%$, bootstrapped $n=999)$ for the bi-seasonal reflective variable 
set. For random forest models, achievable accuracy for the same variable sets was estimated to be about $13 \%$ higher, with $74.5 \%(C I: 73.8-75.2 \%$, bootstrapped $n=999)$ for the dry season and $90.6 \%$ for the bi-seasonal reflective variable set (CI: $89.6-91.4 \%$, bootstrapped $n=999$ ) (Fig. 3.2). Linear-log regression models for overall accuracy as a function of sampling intensity by variable set indicated that, in the case of random forest models, doubling the sampling intensity increased overall accuracy by $2.5 \%\left(R^{2}=0.86\right)$ for the dry season, by $3.1 \%\left(R^{2}=0.76\right)$ for the wet season and by $2.5 \%\left(R^{2}=0.78\right)$ for the combined seasons. For the naïve Baye classifiers, the increase in accuracy ranged between only $0.8 \%$ and $1 \%$ when doubling training samples ( $R^{2}$ ranging from 0.21 to 0.4$)$.

\section{Training and Prediction Time}

Training time for the two classifier methods as a function of number of training samples, number of variables, and number of classes indicated that for both classifiers, all three variables were significant $(p<0.001)$. Classifier training times were significantly faster for all random forest models when compared to naïve Bayes models for equal sampling intensities and number of variables. Average speed across all models was twice as fast for random forest models $(0.51 \pm 0.19)$ (Fig. 3.3). For the naïve Bayes classifier, doubling the number of training samples increased average training time by $57 \%$ (Fig. 3.3), but adding another image or texture increased training time by $98 \%$; doubling the number of classes increased it by $206 \%\left(R^{2}=0.98 ; p<0.001\right)$ (Fig 3.3). For the random forest models, doubling the number of training samples increased training times on average by 86\%. Doubling the feature space by adding an additional image or including texture for each spectral band increased training times by $89 \%$ (Fig. 3.3), and doubling the number of 
classes increased it by $106 \%\left(R^{2}=0.98 ; p<0.001\right)$ when each of the other two variables was held constant (Fig 3.3).

Prediction times were affected most for the naïve Bayes classifier when a kernel estimator was used during the tuning process (Fig. 3.4). Prediction times for kernel estimator models were mostly affected by number of optimal features selected and number of classes, where doubling the number of features increased prediction time by $91 \%$ and doubling the number of classes increased prediction times on average by $85 \%\left(R^{2}=0.99 ; p\right.$ $<0.001$ ) (Fig 3.4). Naïve Bayes models that did not use kernel estimates saw an average increase in prediction time by $3.5 \%$ when the selected features increased by one and by $7 \%$ for doubling the number of classes $\left(R^{2}=0.95 ; p<0.001\right)$ (Fig 3.4). Random forest prediction times were not correlated with number of features selected during the feature selection process of the training process or with number of classes $\left(R^{2}=0.07 ; p<0.001\right)$ (Fig. 3.4). Since overall accuracy for random forests was consistently higher for all variable sets, and training and prediction times were less affected by feature space and sampling intensity, class-specific accuracy and location-specific confidence were evaluated for random forest models, only.

\section{Location-specific Confidence Estimates}

Aggregated area of confidence $\geq 90 \%$ increased with sampling intensity for all variable sets in the range of the evaluated sampling intensities (Fig. 3.5, Tbl. 3.6). Similar to overall accuracy, confidence had a diminished return with increase in sampling intensity. Increasing sampling intensity from 200 to 300 samples reduced the gain in confidence to less than $3 \%$ and for wet and bi-seasonal data to less than 2\% (Fig. 3.5, Tbl. 3.6). 
Test results for one-sided pairwise-paired Wilcoxon signed-rank tests indicated that increasing sampling intensities and number of features in variable sets increased locationspecific confidence $(p<0.001)$. The same was observed when using wet season vs. dry season data $(p<0.001)$. The only exceptions were bi-seasonal vs. bi-seasonal + texture for sampling intensities of 12 and 50 samples, where the fewer features (no texture) had a significantly higher number of pixels with higher confidence $(p<0.001)$, which was also observed in the increased area of aggregated confidence $\geq 90 \%$ (Fig. 3.5, Tbl. 3.6).

The pattern of local variability of location-specific confidence for the bi-seasonal + texture dataset and for three confidence thresholds $(\geq 90 \% ; 50 \leq x<90 \% ;<50 \%)$ showed that even at low sampling intensities of 12 samples, only $2.4 \%$ of the area had a confidence of less than $50 \%$, but only $55 \%$ had a confidence of at least $90 \%$ (Fig. 3.6). Increasing sampling intensity to 25 reduced the area with confidence $\leq 50 \%$ by $50 \%$ and increased the high confidence area $\geq 90 \%$ by $14 \%$ (Fig. 3.6, Tbl. 3.6). Doubling sampling intensity for bi-seasonal data, the percent of area with location-specific confidence $\geq 90 \%$ increased by $5.8 \pm 0.83 \%\left(R^{2}=0.95 ; p<0.001\right)$; doubling sampling intensity for individual wet and dry season increased this area by $7.0 \pm 1.1 \%\left(R^{2}=0.95 ; p<0.001\right)$ and $6.3 \pm 0.86 \%\left(R^{2}=0.96\right.$; $p<0.001)$, respectively.

Overall, bi-seasonal data on average produced a $2.6 \%(S D= \pm 1.35 \%, p<0.001)$ increase in area with spatial confidence $\geq 90 \%$ when compared to the wet season and a $15.4 \%$ increase in area $(S D= \pm 3.34 \%, p<0.001)$ when compared to the dry season. Overall accuracy and location-specific confidence analysis suggest that a sampling intensity of 200 samples is a good cut-off point; beyond this sampling intensity, the increase in accuracy was reduced to $0.8 \%(S D= \pm 0.001 \%)$ and confidence to $1.9 \%(S D= \pm 0.59 \%)$. Class- 
specific user's and producer's accuracies were therefore evaluated only for sampling intensity of 200 samples.

Class-specific User's and Producer's Accuracy

Class-specific user's and producer's accuracies for the six variable sets are presented in Table 3.7. Mean accuracy for all classes and variable sets was $86.3 \%(S D= \pm 14 \%)$ for producer's accuracy and $84.2 \%(S D= \pm 15.7 \%)$ for user's accuracy. Mean standard error was $0.8 \%(S D= \pm 0.4 \%$ ) for both producer's and user's accuracies (Fig. 3.7 and 3.8, Tbl. 3.7).

\section{$\underline{\text { Single season comparison }}$}

A comparison of user's accuracies indicated that there was a significant difference between wet and dry season data for all classes (Tbl. 3.8). For two of the four slough classes ("Submerged Aquatic" and "Floating Broadleaf / Submerged Aquatic") and one of the four ridge classes (“Shrub / Graminoid Marsh / Emergent Broadleaf”), the dry season had significantly higher accuracies, whereas the other five classes had higher accuracies for the wet season data (Tbl. 3.8; Figs. 3.7 and 3.8). Dry season accuracies for these three classes were $30.4 \%$ (CI: $27.6-32 \%$ ), $4.8 \%$ (CI: $2.3-7.3 \%$ ), and $25.8 \%$ (CI: $21.6-29.1 \%$ ) higher than for the wet season (Tbl. 3.8).

Wet season data had a significantly greater user's accuracy for the other 5 classes, ranging from $9.6 \%$ (CI: $7.1-13.1 \%$ ) greater for "Nymphaea" to $59.2 \%$ (CI: $54.5-63.9 \%)$ greater for the "Shrub / Tree" class (Tbl. 3.8). The "Shrub / Tree" class had the lowest user's accuracy of the dry season with $34.6 \%(S E= \pm 0.8 \%)$, followed by "Graminoid Marsh / Emergent Broadleaf" mix class with 43.7\% $(S E= \pm 0.9 \%)$ accuracy (Tbl. 3.7). These classes were detected from wet season data at a mean accuracy of 93.7\% $(S E= \pm 1.7 \%)$ and 
$69.9 \%(S E= \pm 1.1 \%)$, respectively. A similar overall pattern of variable set and class detectability was observed for producer's accuracy except for the "Submerged Aquatic" class, which had a slightly higher accuracy for the wet season data $(2.9 \%$ (CI: $1.3-4.2 \%)$, significant at $\alpha=0.05$ ) and the "Nymphaea" class, where differences were not statistically significant (Tbl. 3.9).

\section{$\underline{\text { Bi-seasonal data }}$}

Combining wet and dry season signatures significantly increased user's accuracies for predicting wet or dry class labels for all ridge classes when compared to wet or dry season signatures only (Tbl. 3.8, Fig. 3.7). The largest increase of 64.1\% (CI: $62.2-66.7 \%)$ was observed for the "Shrub / Tree" class when compared to dry season data, followed by $42.3 \%$ (CI: $39.9-44.4 \%), 20.6 \%(C I: 18.6-21.9 \%)$ and $11.8 \%(C I: 10-13.7 \%)$ for the “Graminoid Marsh / Emergent Broadleaf”, “Cladium”, and the "Shrub / Graminoid Marsh / Emergent Broadleaf' classes, respectively. The class that benefitted most from the biseasonal data when compared to wet season only was the "Shrub / Graminoid Marsh / Emergent Broadleaf", which increased by 37.9\% (Tbl. 3.8, Fig. 3.7).

For the slough classes, when predicted from bi-seasonal data, the largest increase of $18.8 \%(C I: 16.4-23.5 \%)$ was recorded for the dry season class label "Submerged Aquatic / Periphyton / Floating Broadleaf" when compared to dry season data only (Tbl. 3.8, Fig. 3.8). However, accuracy of the same class was reduced by $0.7 \%(C I: 0.3-1 \%)$ when compared to the wet season data, a small but statistically significant reduction. An even larger accuracy reduction was observed for the "Floating Broadleaf / Submerged Aquatic" class, where bi-seasonal data reduced accuracy by 4.7\% (CI: $2.4-7 \%$ ) (Tbl. 3.8, Fig. 3.8). 
The "Nymphaea" class experienced an approximately $3 \%$ increase when predicting from biseasonal vs. single wet or dry season data (Tbl. 3.8, Fig. 3.8).

Producer's accuracy increased significantly when using bi-seasonal vs. dry season data for all classes except the two slough classes "Submerged Aquatic" and "Floating Broadleaf / Submerged Aquatic", for which the differences were insignificant (Tbl. 3.9). When compared to wet season data only, bi-seasonal data improved the "Shrub / Graminoid Marsh / Emergent Broadleaf" by 41.1\% (CI: 38 -45.7\%) (Tbl. 3.9, Fig. 3.7). The other ridge class benefiting from the bi-seasonal signature was the "Cladium" class. The two slough classes for which the producer's accuracies increased significantly with bi-seasonal data were "Submerged Aquatic / Periphyton / Floating Broadleaf" and "Floating Broadleaf / Submerged Aquatic" (Tbl. 3.9, Fig. 3.8).

\section{$\underline{\text { Texture }}$}

Adding local texture variables for a single season did not affect user's accuracies significantly (Tbl. 3.8, Figs. 3.7 and 3.8). Only "Graminoid Marsh / Emergent Broadleaf" had a statistically significant increase of $1.9 \%(\alpha=0.05)$ for the dry season data. For biseasonal data, texture improved user's accuracy for the ridge class "Graminoid Marsh / Emergent Broadleaf" by 2.2\% $(\alpha=0.05)$ (Tbl. 3.8, Fig. 3.7). For the slough class "Floating Broadleaf / Submerged Aquatic", adding texture produced a 3.7\% $(\alpha=0.001)$ increase for the wet season and a 2.3\% $(\alpha=0.01)$ reduction for the dry season (Tbl. 3.8, Fig. 3.8). Texture only affected producer's accuracy for the ridge class "Graminoid Marsh / Emergent Broadleaf" for the wet season data, increasing it by $4.2 \%(\alpha=0.05)$ (Tbl. 3.9, Fig. 3.7). For the slough class "Floating Broadleaf / Submerged Aquatic", the reversed pattern to user's 
accuracy was observed: for the bi-seasonal data, as texture was added, accuracy dropped by $1.9 \%(\alpha=0.01)$ and increased by $2.4 \%(\alpha=0.05)$ for the dry season (Tbl. 3.9, Fig. 3.8).

\section{DISCUSSION}

Maximizing map classification accuracy and confidence is the primary goal when mapping land-cover from remotely sensed data, and knowledge of class-specific as well as spatially explicit errors and uncertainties are essential for propagation of errors and uncertainties in subsequent models that incorporate these maps. Evaluating and selecting mapping methods that promise high accuracies at high spatial resolution (precision) while keeping production costs low is especially important when mapping large spatial extents at multiple time-intervals. Many monitoring programs rely on high-precision class detection and mapping products. Results of this study show that the comparison of mapping methods

on the basis of overall accuracy, confidence estimates, and classifier efficiency allows for optimization of mapping methods. In this study, alternative models were evaluated in an integrated way using a framework that considered not only sampling intensities and variable sets but also their interactions and their effects on accuracy, confidence, and classifier efficiency. Significance of performance differences was tested, and confidence intervals for difference estimates were established.

\section{Classifier Performance}

Classifier performance and efficiency depend on evaluation criteria and vary across different disciplines and subject domains, indicating that there is no inherently superior classification method that consistently minimizes variance and/or bias of accuracy estimates (Duda, Hart, and Stork 2001). Evaluations of classification methods across different disciplines, however, suggests that parametric classifiers such as naïve Bayes can be more 
efficient than machine learning algorithms (Xhemali, Hind, and Stone 2009). The results of this study demonstrated that overall map accuracy was significantly higher for the random forest models and, therefore, out-performed naïve Bayes classifiers.

Training times for random forest and naïve Bayes were affected by sampling intensity and class and feature numbers in different ways. While doubling training sample size had a greater impact on the percent increase in training time for random forest algorithms, random forest algorithms had faster absolute training times when compared to naïve Bayes classifiers for every model run across all sampling intensities. Doubling the feature space and increasing class number affected training times for naïve-Bayes models more than random forest models. However, these results could be an artifact of the implementation of the algorithms as they are implemented and programmed in the $\mathrm{R}$ packages that were used for this analysis. Training times for either algorithm never exceeded 12 minutes even for 300 training samples per class and is, therefore, not a major concern for applications. Prediction times, however, are of concern as the study area extent increases. For the naïve-Bayes classifier, especially when kernel density estimators were used, prediction times increased rapidly and could become unfeasible for large mapping areas. For example, classifying a $100 \mathrm{~km}^{2}$ study area using bi-seasonal WV-2 data with a spatial resolution of $2 \mathrm{~m}$ can be processed with the test computer in approximately 3.5 hours but could take more than 100 hours when using the naïve-Bayes classifier and months if using the tuning parameter and kernel density estimators. This demonstrates that algorithm evaluation is crucial when selecting appropriate methods for large mapping projects or monitoring programs. Both data processing time and accuracy need to be considered. 
Since random forest classifier training and prediction times were virtually unaffected by number of features or classes, training sample digitization and data acquisition costs are the determining factors that should be considered for method evaluations when using random forest classifiers. Increasing sampling intensity requires an increase in digitization time and often an increase in associated field visits, which are costly, as they require an increase in human work hours and transportation costs. If the addition of a second image acquired at a different time increases accuracy, as shown in this study, it might be worthwhile to invest in more frequent data acquisition rather than increased sampling intensity.

\section{Bi-Seasonal Data}

In this study, overall accuracy significantly increased with bi-seasonal data for both classifiers and regardless of sampling intensities for naïve Bayes classifiers and random forest models. This can be explained by the differential spectral response of vegetation classes during the wet and dry seasons. The seasonal variability is mainly due to the hydrological and plant specific phenological cycles. These intra-annual variabilities contribute to confusion and can lead to misclassification of vegetation classes at a single time, but they can also assist in the identification of otherwise confounded vegetation types when multi-temporal data are used (Gilmore et al. 2008). Vegetation phenology and hydrological cycles play a major role in selecting optimal multi-seasonal remotely sensed data (features) with the purpose to maximize phenological differences between vegetation types that otherwise are confused. In the case of wetlands, it has been demonstrated that detection of wetland communities and vegetation structure (Davranche, Lefebvre, and Poulin 2010; Poulin, Davranche, and Lefebvre 2010) and spectral distinction of species at 
different times throughout a year (Gao and Zhang 2006; Gilmore et al. 2008) can increase with strategically chosen images that maximize the intra-annual, between-class spectral variability. Intra-annual variability of wetland vegetation benefits from incorporating phenological cycles in the data selection process, especially for communities that include sub-canopy vegetation or seasonally shifting species dominance (e.g., shifts in grassdominated vs. sedge- or rush-dominated communities throughout the year) and growth densities, or as in the case of this study, floating and seasonally occurring slough vegetation components.

The presented results also indicate that increase in sampling intensity boosts accuracies in a reasonably predictable fashion. However, the addition of a second image acquired at a time when ground conditions and phenological differences in vegetation types are observed had a much higher impact on classification accuracy, increasing accuracy even at very low sampling intensities to levels that were never reached with a single season image. If bi-seasonal data acquisition is not possible, knowing which timeframe maximizes single season imagery is crucial as the second-best option. Wet-season data provided the second-best solution, but the analysis also showed that some classes benefitted more from dry-season data, which emphasizes the need to consult class-specific user's and producer's accuracies when specific classes are of higher priority than others.

Vegetation that was consistent or stable between the two seasons (same class label) benefitted from the use of a bi-seasonal dataset. For instance, utilizing the phenological cycles of the vegetation improved the separation of shrub and broadleaved mix classes, which were confused in single season imagery. During the late dry season, the time when several shrub and tree species have new leaves, the abundance of the shrub/tree class was 
over-predicted, while at the end of the wet season, when they shed their leaves, the models under-predicted this class and committed it to the broadleaf emergent and graminoid marsh classes. The combined signature benefitted from the exposed understory signature of the tree and shrub classes during the late wet season. If high seasonal variability in phenology across classes exists, using more than two images could further increase accuracies of some classes. If those classes are common classes across the landscape, the effect on spatial accuracy distribution and location-specific confidence across the landscape will be affected as well. Significance of such changes on class-specific accuracy and location-specific confidence needs to be investigated.

For vegetation classes that exist in a highly variable and dynamic seasonal environment, multi-seasonal data not only allow for estimation of surface cover, taking advantage of capturing phenology, but also make it possible to estimate percent cover change of classes between dates. Seasonal variability of slough communities, for instance, when estimated from bi-seasonal mapped classes showed that within this study area, periphyton areal cover was $12.6 \%(C I: 12.0-13.2 \%)$ for the dry season and $21.2 \%(C I$ : $20.7-21.7 \%$ ) for the wet season, with $10.8 \%$ (CI: $10.5-11.1 \%$ ) of the study area covered by periphyton during both seasons on the days of data acquisition. Using multiple images in the analysis allows for making spatially explicit maps of changes between dates with high accuracy and spatial precision. Highly variable and dynamic classes, therefore, benefit from datasets acquired at multiple times throughout a year. This is especially an important factor when attempting to derive dynamics of spatial patterns of phenomena with high intra- and inter-annual variability. For instance, modeling nutrient gradients and hydrological changes and their impacts on the production and decomposition of periphyton could be possible, 
because seasonal and inter-annual patterns of variability can be tracked using multitemporal remotely sensed data over large extents.

Categorical maps are generally accompanied by metadata that provide only overall accuracy, and at the most, error matrices or class-specific user's and producer's accuracies, but not location-specific confidence estimates of class membership probability as determined by the classifier. Location-specific confidence maps that accompany categorical maps can be incorporated into spatially explicit ecological models and, therefore, contribute to estimating model output confidence in a spatially explicit manner. Further study is needed to determine if location-specific confidence is correlated with classspecific accuracy, and what factors determine spatial patterns of location-specific confidence. Since a common method to derive categorical maps is by application of supervised classification methods to remotely sensed data, estimation of spatially explicit accuracy and confidence should become an integral part of the map production process.

The integrated framework using a training data re-sampling method to determine sampling error was useful in establishing and comparing classifier consistency and modeling classifier accuracy and efficiency as a function of training sample size and feature space. Since no single classification method maximizes overall and class-specific accuracies across all classes of interest, quantifying cost associated with alternative methods is crucial for the selection of the optimal classification method, and needs to be evaluated in the context of specific project goals. The use of linear and non-linear regression models demonstrated that it was possible to estimate accuracy changes and predict upper accuracy limits as a function of sampling intensity and for different feature-space subsets. These results indicate that a re-sampling framework is useful to model and predict expected overall 
map accuracies for different resource allocation strategies - e.g., multi-seasonal analysis vs. increase in sampling intensities. The regression results show that the predictive power is very high with coefficients of determination for all models greater than $0.95(p<0.001)$. These types of models are useful for estimating mapping costs and accuracy return for alternative investments. 


\section{TABLES}

Table 3.1. Classification scheme for the high-resolution plant community maps of the ridge and slough landscape in Water Conservation Area 3A (Fig. 3.1).

\section{Morphological}

Class

\section{Community Class Name}

Submerged Aquatic

Slough Submerged Aquatic / Periphyton / Floating Broadleaf

Floating Broafleaf / Submerged Aquatic

Floating Broadleaf Nymphaea

Graminoid Marsh / Emergent Broadleaf

Ridge Graminoid marsh Cladium

Shrub / Graminoid Marsh / Emergent Broadleaf

Shrub / Tree

Table 3.2. The 72 model sets for each combination of a full factorial design of two classifiers, six variable sets and six sampling intensities $(2 \times 6 \times 6)$.

\begin{tabular}{c|c|c}
\hline \multicolumn{1}{c}{ Classifier } & Variable Set & Sampling Intensity \\
\hline \hline \multirow{4}{*}{ Random Forest } & Dry & 12 \\
& Dry + Texture & 25 \\
& Wet & 50 \\
\multirow{4}{*}{ Naïve Bayes } & Wet + Texture & 100 \\
& Wet \& Dry & 200 \\
& Wet \& Dry + Texture & 300 \\
\hline
\end{tabular}


Table 3.3. Comparison of overall accuracy for naïve Bayes and random forest classifiers by variable set and sampling intensity. Var. = Variable; Samp. Int. = Sampling Intensity; Diff. Loc. = Estimated Median Location Difference; $\mathbf{C I}=$ Confidence Interval; Sign. = Significance, where -, *, ** and $* * *$ indicate not significant, significant at alpha $<0.05,0.01$ and 0.001 , respectively; $\mathbf{T x t}=$ Texture.

\begin{tabular}{|c|c|c|c|c|c|c|c|c|c|c|c|c|}
\hline \multirow[b]{2}{*}{$\begin{array}{l}\text { Var. } \\
\text { Set }\end{array}$} & \multirow[b]{2}{*}{$\begin{array}{l}\text { Samp. } \\
\text { Int. }\end{array}$} & \multicolumn{3}{|c|}{ naïve Bayes } & \multicolumn{3}{|c|}{ Random Forest } & \multicolumn{5}{|c|}{ Mann-Whitney Test } \\
\hline & & Mdn. & $\begin{array}{c}\text { CI - } \\
\text { Lower }\end{array}$ & $\begin{array}{c}\text { CI - } \\
\text { Upper }\end{array}$ & Mdn. & $\begin{array}{c}\text { CI - } \\
\text { Lower }\end{array}$ & $\begin{array}{c}\text { CI - } \\
\text { Upper }\end{array}$ & $\begin{array}{l}\text { Diff. } \\
\text { Loc. }\end{array}$ & $\begin{array}{c}\text { CI - } \\
\text { Lower }\end{array}$ & $\begin{array}{c}\text { CI - } \\
\text { Upper }\end{array}$ & $\begin{array}{c}\text { p- } \\
\text { value }\end{array}$ & Sign. \\
\hline \multirow{6}{*}{$\vec{\theta}$} & 12 & $57.5 \%$ & $53.4 \%$ & $60.8 \%$ & $62.8 \%$ & $60.6 \%$ & $65.4 \%$ & $-5.5 \%$ & $-7.6 \%$ & $-3.2 \%$ & $<0.000$ & **** \\
\hline & 25 & $9.8 \%$ & $58.1 \%$ & $62.6 \%$ & $8.0 \%$ & $62.8 \%$ & $70.2 \%$ & $-7.9 \%$ & $-9.9 \%$ & $-5.4 \%$ & $<0.000$ & $* * *$ \\
\hline & 50 & $0.5 \%$ & $59.5 \%$ & $62.5 \%$ & $0.6 \%$ & $68.9 \%$ & $71.7 \%$ & $-9.4 \%$ & $-10.5 \%$ & $-8.4 \%$ & $<0.000$ & $* * *$ \\
\hline & 100 & $0.3 \%$ & $58.2 \%$ & $61.4 \%$ & $72.6 \%$ & $71.1 \%$ & $73.7 \%$ & $-12.3 \%$ & $-13.3 \%$ & $-11.5 \%$ & $<0.000$ & $* * *$ \\
\hline & 200 & $2.0 \%$ & $60.8 \%$ & $62.5 \%$ & $74.7 \%$ & $73.4 \%$ & $75.4 \%$ & $-12.8 \%$ & -13 & $-12.0 \%$ & $<0.000$ & $* * *$ \\
\hline & 300 & $61.7 \%$ & $60.1 \%$ & $62.7 \%$ & $75.1 \%$ & $73.5 \%$ & $75.5 \%$ & $-13.3 \%$ & $-13.9 \%$ & $-12.1 \%$ & $<0.000$ & $* * *$ \\
\hline \multirow{6}{*}{$\begin{array}{l}\vec{A} \\
\vec{\theta} \\
+ \\
\vec{\Delta}\end{array}$} & 12 & $9.5 \%$ & $53.9 \%$ & $61.2 \%$ & $61.2 \%$ & $57.2 \%$ & $64.8 \%$ & $-3.0 \%$ & $-5.2 \%$ & $0.2 \%$ & 0.064 & - \\
\hline & 25 & $61.9 \%$ & $56.0 \%$ & $64.1 \%$ & $8.3 \%$ & $65.0 \%$ & $69.6 \%$ & $-6.3 \%$ & $-8.5 \%$ & $-4.6 \%$ & $<0.000$ & $* * *$ \\
\hline & 50 & $61.8 \%$ & $59.2 \%$ & $63.5 \%$ & $69.8 \%$ & $68.5 \%$ & $71.3 \%$ & $-8.1 \%$ & $-9.8 \%$ & $-7.1 \%$ & $<0.000$ & $* * *$ \\
\hline & 100 & $61.9 \%$ & $59.3 \%$ & $62.7 \%$ & $72.4 \%$ & & $73.3 \%$ & $-10.7 \%$ & $-11.6 \%$ & $-9.8 \%$ & $<0.000$ & $* * *$ \\
\hline & 200 & $62.3 \%$ & $61.9 \%$ & $63.6 \%$ & $73.6 \%$ & $72.6 \%$ & $74.6 \%$ & $-11.2 \%$ & -11 & $-10.4 \%$ & $<0.000$ & $* * *$ \\
\hline & 300 & $62.0 \%$ & $61.1 \%$ & $62.8 \%$ & $74.3 \%$ & 73. & $75.5 \%$ & $-12.4 \%$ & -13 & $-11.8 \%$ & $<0.000$ & $* * *$ \\
\hline \multirow{6}{*}{$\sum^{0}$} & 12 & $67.5 \%$ & $64.2 \%$ & $70.5 \%$ & $74.3 \%$ & 66. & $79.2 \%$ & $-7.3 \%$ & & $-3.1 \%$ & 0.001 & $* *$ \\
\hline & 25 & $67.2 \%$ & $64.3 \%$ & $72.5 \%$ & $78.4 \%$ & 73.7 & $83.9 \%$ & $-10.2 \%$ & -13 & $-7.1 \%$ & $<0.000$ & $* * *$ \\
\hline & 50 & $.6 \%$ & $64.6 \%$ & $71.9 \%$ & $2.2 \%$ & 79.3 & $84.6 \%$ & $-13.4 \%$ & & $-10.9 \%$ & $<0.000$ & \\
\hline & 100 & $.1 \%$ & $69.1 \%$ & $71.9 \%$ & $85.8 \%$ & 83. & $87.0 \%$ & $-15.4 \%$ & & $1 \%$ & $<0.000$ & $* * *$ \\
\hline & 200 & $0.9 \%$ & $66.9 \%$ & $73.1 \%$ & $7.1 \%$ & & $88.7 \%$ & $-16.2 \%$ & & & $<0$. & $* * *$ \\
\hline & 300 & $70.5 \%$ & $69.3 \%$ & $72.6 \%$ & $8.6 \%$ & & & $-17.5 \%$ & & -16 & $<0$. & $* * *$ \\
\hline \multirow{6}{*}{$\begin{array}{l}\vec{t} \\
\vec{A} \\
+ \\
\overrightarrow{0} \\
\overrightarrow{0}\end{array}$} & 12 & $65.5 \%$ & $62.4 \%$ & $72.9 \%$ & $73.9 \%$ & $69.9 \%$ & $79.8 \%$ & $-7.8 \%$ & -11 & $-3.0 \%$ & 0.0 & $* *$ \\
\hline & 25 & $67.2 \%$ & $63.3 \%$ & $70.3 \%$ & $77.8 \%$ & $73.2 \%$ & $79.5 \%$ & $-10.0 \%$ & $-12.4 \%$ & $-7.6 \%$ & $<0.000$ & $* * *$ \\
\hline & 50 & $67.9 \%$ & $64.9 \%$ & $73.4 \%$ & $82.1 \%$ & $79.9 \%$ & $83.5 \%$ & $-13.6 \%$ & -16.4 & $-10.3 \%$ & $<0.000$ & $* * *$ \\
\hline & 100 & $70.7 \%$ & $68.6 \%$ & $73.4 \%$ & $5.6 \%$ & $83.6 \%$ & $86.5 \%$ & $-14.2 \%$ & -15 & $-12.9 \%$ & $<0.000$ & $* * *$ \\
\hline & 200 & $70.0 \%$ & $68.4 \%$ & $72.2 \%$ & $7.1 \%$ & $86.5 \%$ & $88.7 \%$ & $-17.3 \%$ & -18 & $-16.4 \%$ & $<0.000$ & $* * *$ \\
\hline & 300 & $69.9 \%$ & $68.1 \%$ & $71.9 \%$ & $87.7 \%$ & $87.1 \%$ & $88.3 \%$ & $-17.8 \%$ & $-19.0 \%$ & $-16.2 \%$ & $<0.000$ & $* * *$ \\
\hline \multirow{6}{*}{$\begin{array}{l}\overrightarrow{0} \\
\dot{0} \\
\dot{0} \\
\overrightarrow{0}\end{array}$} & 12 & $73.3 \%$ & $68.2 \%$ & $76.0 \%$ & $80.4 \%$ & $72.7 \%$ & $83.1 \%$ & $-7.6 \%$ & $-10.2 \%$ & $-4.5 \%$ & 0.003 & $* *$ \\
\hline & 25 & $74.9 \%$ & $68.8 \%$ & $78.0 \%$ & $3.1 \%$ & 78.9 & $85.7 \%$ & $-9.2 \%$ & -12 & $-5.9 \%$ & $<0.000$ & $* * *$ \\
\hline & 50 & $73.8 \%$ & $72.0 \%$ & $76.8 \%$ & $86.9 \%$ & $85.0 \%$ & $88.8 \%$ & $-12.8 \%$ & $-14.5 \%$ & $-11.2 \%$ & $<0.000$ & $* * *$ \\
\hline & 100 & $75.6 \%$ & $72.0 \%$ & $77.2 \%$ & $89.0 \%$ & $88.0 \%$ & $90.1 \%$ & $-13.7 \%$ & -16 & $-12.4 \%$ & $<0.000$ & $* * *$ \\
\hline & 200 & $76.7 \%$ & $74.2 \%$ & $78.4 \%$ & $90.6 \%$ & $89.8 \%$ & $91.3 \%$ & $-13.9 \%$ & -15 & $-13.3 \%$ & $<0.000$ & $* * *$ \\
\hline & 300 & $76.7 \%$ & $75.2 \%$ & $77.7 \%$ & $90.7 \%$ & $89.9 \%$ & $91.2 \%$ & $-14.0 \%$ & -14 & $-13.4 \%$ & $<0.000$ & $* * *$ \\
\hline \multirow{6}{*}{ 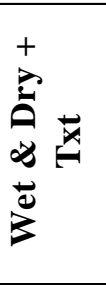 } & 12 & $69.3 \%$ & $63.8 \%$ & $75.9 \%$ & $77.0 \%$ & $71.7 \%$ & $84.3 \%$ & $-7.3 \%$ & -12 & $-2.4 \%$ & 0.001 & $* *$ \\
\hline & 25 & $72.1 \%$ & $64.9 \%$ & $75.6 \%$ & $82.9 \%$ & $80.3 \%$ & $85.2 \%$ & $-11.4 \%$ & $-14.8 \%$ & $-8.4 \%$ & $<0.000$ & $* * *$ \\
\hline & 50 & $70.7 \%$ & $68.8 \%$ & $75.1 \%$ & $86.0 \%$ & $84.4 \%$ & $87.6 \%$ & $-15.5 \%$ & $-16.9 \%$ & $-13.2 \%$ & $<0.000$ & $* * *$ \\
\hline & 100 & $73.6 \%$ & $71.9 \%$ & $75.0 \%$ & $88.8 \%$ & $87.7 \%$ & $89.7 \%$ & $-15.1 \%$ & $-16.1 \%$ & $-14.2 \%$ & $<0.000$ & $* * *$ \\
\hline & 200 & $74.3 \%$ & $72.1 \%$ & $76.0 \%$ & $89.9 \%$ & $88.9 \%$ & $91.1 \%$ & $-16.0 \%$ & $-17.4 \%$ & $-14.6 \%$ & $<0.000$ & $* * *$ \\
\hline & 300 & $74.5 \%$ & $72.5 \%$ & $76.4 \%$ & $91.2 \%$ & $90.3 \%$ & $91.8 \%$ & $-16.5 \%$ & $-17.8 \%$ & $-15.6 \%$ & $<0.000$ & $* * *$ \\
\hline
\end{tabular}


Table 3.4. Comparison of overall accuracy differences for the naïve Bayes classifier when comparing variable sets by sampling intensity. Var. $=$ Variable; Smp. Int. $=$ Sampling Intensity; Est. Diff. = Estimated Difference; Conf. = Confidence Interval; Sign. = Significance, where,$- * * *$ and $* * *$ indicate not significant, significant at alpha $<0.05,0.01$ and 0.001 , respectively; $T x t=$ Texture.

\begin{tabular}{|c|c|c|c|c|c|c|}
\hline \multirow{2}{*}{$\frac{\text { Var. Set } 1}{\text { Dry }}$} & \multirow{2}{*}{$\frac{\text { Var. Set } 2}{\text { Wet }}$} & \multirow{2}{*}{$\begin{array}{c}\text { Smp. Int. } \\
12\end{array}$} & \multirow{2}{*}{$\begin{array}{c}\text { Est. Diff. } \\
-9.74 \%\end{array}$} & \multicolumn{2}{|c|}{ 95\% Conf. } & \multirow{2}{*}{$\begin{array}{r}\text { Sign. } \\
* * *\end{array}$} \\
\hline & & & & $-12.36 \%$ & $-7.24 \%$ & \\
\hline Dry & Wet & 25 & $-7.60 \%$ & $-10.32 \%$ & $-5.57 \%$ & $* * *$ \\
\hline Dry & Wet & 50 & $-8.04 \%$ & $-10.48 \%$ & $-5.40 \%$ & $* * *$ \\
\hline Dry & Wet & 100 & $-9.92 \%$ & $-11.16 \%$ & $-9.08 \%$ & $* * *$ \\
\hline Dry & Wet & 200 & $-9.06 \%$ & $-10.00 \%$ & $-7.80 \%$ & $* * *$ \\
\hline Dry & Wet & 300 & $-8.86 \%$ & $-10.08 \%$ & $-7.92 \%$ & $* * *$ \\
\hline Dry & Dry + Txt & 12 & $-1.16 \%$ & $-3.76 \%$ & $1.36 \%$ & - \\
\hline Dry & Dry + Txt & 25 & $-1.74 \%$ & $-3.72 \%$ & $0.64 \%$ & - \\
\hline Dry & Dry + Txt & 50 & $-1.03 \%$ & $-2.16 \%$ & $0.64 \%$ & - \\
\hline Dry & Dry + Txt & 100 & $-1.55 \%$ & $-2.32 \%$ & $-0.48 \%$ & $* *$ \\
\hline Dry & Dry + Txt & 200 & $-0.52 \%$ & $-1.32 \%$ & $0.00 \%$ & $*$ \\
\hline Dry & Dry + Txt & 300 & $-0.38 \%$ & $-1.07 \%$ & $0.20 \%$ & - \\
\hline Wet & $\mathrm{Wet}+\mathrm{Txt}$ & 12 & $0.81 \%$ & $-3.44 \%$ & $4.00 \%$ & - \\
\hline Wet & Wet + Txt & 25 & $0.48 \%$ & $-2.08 \%$ & $3.72 \%$ & - \\
\hline Wet & Wet + Txt & 50 & $-0.16 \%$ & $-3.04 \%$ & $3.00 \%$ & - \\
\hline Wet & Wet + Txt & 100 & $-0.80 \%$ & $-1.93 \%$ & $0.52 \%$ & - \\
\hline Wet & Wet + Txt & 200 & $0.84 \%$ & $-1.48 \%$ & $2.13 \%$ & - \\
\hline Wet & Wet + Txt & 300 & $0.70 \%$ & $-0.92 \%$ & $1.96 \%$ & - \\
\hline Wet & Wet \& Dry & 12 & $-5.60 \%$ & $-8.08 \%$ & $-2.60 \%$ & $* *$ \\
\hline Wet & Wet \& Dry & 25 & $-5.94 \%$ & $-9.48 \%$ & $-2.52 \%$ & $* *$ \\
\hline Wet & Wet \& Dry & 50 & $-5.52 \%$ & $-8.08 \%$ & $-3.00 \%$ & $* * *$ \\
\hline Wet & Wet \& Dry & 100 & $-4.83 \%$ & $-6.24 \%$ & $-2.84 \%$ & $* * *$ \\
\hline Wet & Wet \& Dry & 200 & $-5.66 \%$ & $-7.32 \%$ & $-4.44 \%$ & $* * *$ \\
\hline Wet & Wet \& Dry & 300 & $-6.00 \%$ & $-6.92 \%$ & $-5.04 \%$ & $* * *$ \\
\hline Dry & Wet \& Dry & 12 & $-15.20 \%$ & $-18.12 \%$ & $-12.40 \%$ & $* * *$ \\
\hline Dry & Wet \& Dry & 25 & $-14.36 \%$ & $-16.80 \%$ & $-10.60 \%$ & $* * *$ \\
\hline Dry & Wet \& Dry & 50 & $-13.12 \%$ & $-14.92 \%$ & $-11.72 \%$ & $* * *$ \\
\hline Dry & Wet \& Dry & 100 & $-15.20 \%$ & $-16.43 \%$ & $-12.81 \%$ & $* * *$ \\
\hline Dry & Wet \& Dry & 200 & $-14.72 \%$ & $-15.36 \%$ & $-13.68 \%$ & $* * *$ \\
\hline Dry & Wet \& Dry & 300 & $-15.02 \%$ & $-15.76 \%$ & $-14.16 \%$ & $* * *$ \\
\hline Wet \& Dry & Wet \& Dry + Txt & 12 & $2.61 \%$ & $-1.55 \%$ & $6.79 \%$ & - \\
\hline Wet \& Dry & Wet \& Dry + Txt & 25 & $2.91 \%$ & $-1.16 \%$ & $6.00 \%$ & - \\
\hline Wet \& Dry & Wet \& Dry + Txt & 50 & $3.30 \%$ & $1.24 \%$ & $5.08 \%$ & $* *$ \\
\hline Wet \& Dry & Wet \& Dry + Txt & 100 & $1.57 \%$ & $-0.48 \%$ & $2.88 \%$ & - \\
\hline Wet \& Dry & Wet \& Dry + Txt & 200 & $2.18 \%$ & $1.00 \%$ & $4.12 \%$ & $* *$ \\
\hline Wet \& Dry & Wet \& Dry + Txt & 300 & $2.08 \%$ & $1.04 \%$ & $3.35 \%$ & $* *$ \\
\hline
\end{tabular}


Table 3.5. Comparison of overall accuracy differences for the random forest classifier when comparing variable sets by sampling intensity. Var. = Variable; Smp. Int. = Sampling Intensity; Est. Diff. = Estimated Difference; Conf. = Confidence Interval; Sign. = Significance, where,$- * * *$ and $* * *$ indicate not significant, significant at alpha $<0.05,0.01$ and 0.001 , respectively; $T x t=$ Texture.

\begin{tabular}{|c|c|c|c|c|c|c|}
\hline Var. Set 1 & Var. Set 2 & Smp. Int. & Est. Diff. & \multicolumn{2}{|c|}{ 95\% Conf. } & Sign. \\
\hline Dry & Wet & 12 & $-11.66 \%$ & $-15.32 \%$ & $-7.72 \%$ & $* * *$ \\
\hline Dry & Wet & 25 & $-10.46 \%$ & $-14.56 \%$ & $-7.76 \%$ & $* * *$ \\
\hline Dry & Wet & 50 & $-11.79 \%$ & $-13.27 \%$ & $-10.28 \%$ & $* * *$ \\
\hline Dry & Wet & 100 & $-12.98 \%$ & $-14.04 \%$ & $-12.04 \%$ & $* * *$ \\
\hline Dry & Wet & 200 & $-12.34 \%$ & $-13.60 \%$ & $-11.40 \%$ & $* * *$ \\
\hline Dry & Wet & 300 & $-13.45 \%$ & $-14.12 \%$ & $-12.52 \%$ & $* * *$ \\
\hline Dry & Dry + Txt & 12 & $1.40 \%$ & $-0.79 \%$ & $4.03 \%$ & - \\
\hline Dry & Dry + Txt & 25 & $-0.33 \%$ & $-2.20 \%$ & $1.56 \%$ & - \\
\hline Dry & Dry + Txt & 50 & $0.35 \%$ & $-0.79 \%$ & $1.28 \%$ & - \\
\hline Dry & Dry + Txt & 100 & $0.23 \%$ & $-0.84 \%$ & $1.12 \%$ & - \\
\hline Dry & Dry + Txt & 200 & $0.95 \%$ & $0.24 \%$ & $1.52 \%$ & * \\
\hline Dry & Dry + Txt & 300 & $0.44 \%$ & $-0.48 \%$ & $1.17 \%$ & - \\
\hline Wet & Wet + Txt & 12 & $-0.36 \%$ & $-3.84 \%$ & $4.52 \%$ & - \\
\hline Wet & Wet + Txt & 25 & $0.70 \%$ & $-1.88 \%$ & $4.56 \%$ & - \\
\hline Wet & Wet + Txt & 50 & $0.12 \%$ & $-1.51 \%$ & $1.56 \%$ & - \\
\hline Wet & Wet + Txt & 100 & $0.20 \%$ & $-0.77 \%$ & $1.48 \%$ & - \\
\hline Wet & Wet + Txt & 200 & $-0.36 \%$ & $-1.48 \%$ & $0.68 \%$ & - \\
\hline Wet & Wet + Txt & 300 & $0.53 \%$ & $-0.28 \%$ & $1.28 \%$ & - \\
\hline Wet & Wet \& Dry & 12 & $-4.88 \%$ & $-9.96 \%$ & $-1.52 \%$ & $* *$ \\
\hline Wet & Wet \& Dry & 25 & $-4.62 \%$ & $-7.84 \%$ & $-0.92 \%$ & * \\
\hline Wet & Wet \& Dry & 50 & $-4.80 \%$ & $-6.39 \%$ & $-3.04 \%$ & $* * *$ \\
\hline Wet & Wet \& Dry & 100 & $-3.30 \%$ & $-4.36 \%$ & $-2.44 \%$ & $* * *$ \\
\hline Wet & Wet \& Dry & 200 & $-3.66 \%$ & $-4.68 \%$ & $-2.44 \%$ & $* * *$ \\
\hline Wet & Wet \& Dry & 300 & $-2.24 \%$ & $-3.31 \%$ & $-1.76 \%$ & $* * *$ \\
\hline Dry & Wet \& Dry & 12 & $-17.38 \%$ & $-19.52 \%$ & $-14.84 \%$ & $* * *$ \\
\hline Dry & Wet \& Dry & 25 & $-15.22 \%$ & $-17.56 \%$ & $-11.92 \%$ & $* * *$ \\
\hline Dry & Wet \& Dry & 50 & $-16.39 \%$ & $-18.04 \%$ & $-15.48 \%$ & $* * *$ \\
\hline Dry & Wet \& Dry & 100 & $-16.34 \%$ & $-17.40 \%$ & $-15.48 \%$ & $* * *$ \\
\hline Dry & Wet \& Dry & 200 & $-15.92 \%$ & $-16.68 \%$ & $-15.36 \%$ & $* * *$ \\
\hline Dry & Wet \& Dry & 300 & $-15.76 \%$ & $-16.76 \%$ & $-15.16 \%$ & $* * *$ \\
\hline Wet \& Dry & Wet \& Dry + Txt & 12 & $1.70 \%$ & $-2.48 \%$ & $7.28 \%$ & - \\
\hline Wet \& Dry & Wet \& Dry + Txt & 25 & $0.00 \%$ & $-2.52 \%$ & $2.72 \%$ & - \\
\hline Wet \& Dry & Wet \& Dry + Txt & 50 & $0.72 \%$ & $-0.64 \%$ & $1.84 \%$ & - \\
\hline Wet \& Dry & Wet \& Dry + Txt & 100 & $0.23 \%$ & $-0.55 \%$ & $0.92 \%$ & - \\
\hline Wet \& Dry & Wet \& Dry + Txt & 200 & $0.61 \%$ & $-0.07 \%$ & $1.20 \%$ & - \\
\hline Wet \& Dry & Wet \& Dry + Txt & 300 & $-0.53 \%$ & $-1.05 \%$ & $-0.04 \%$ & $*$ \\
\hline
\end{tabular}


Table 3.6. Location-specific confidence $\geq 90 \%$ aggregated for all pixels across landscape by variable set and sampling intensity. Increase in confidence diminishes with sampling intensity (bottom) (Fig. 3.5); Txt = Texture.

\begin{tabular}{c|cccccc}
\hline \multicolumn{6}{c}{ Aggregated Location-Specific Confidence in Percent } \\
Vampling Intensity \\
Variable Set & $\mathbf{1 2}$ & $\mathbf{2 5}$ & $\mathbf{5 0}$ & $\mathbf{1 0 0}$ & $\mathbf{2 0 0}$ & $\mathbf{3 0 0}$ \\
\hline \hline Dry & 38.6 & 46.1 & 59.9 & 64.4 & 70.1 & 72.3 \\
Dry + Txt & 50.3 & 54.2 & 62.6 & 67.3 & 73.9 & 76.8 \\
\hline Wet & 52.9 & 64.6 & 73.1 & 79.1 & 83.5 & 84.8 \\
Wet + Txt & 58.8 & 66.8 & 74.5 & 80.0 & 83.5 & 85.5 \\
\hline Wet \& Dry & 61.2 & 68.5 & 79.2 & 82.0 & 84.5 & 85.9 \\
Wet \& Dry + Txt & 54.9 & 69.2 & 75.7 & 82.5 & 85.7 & 87.3
\end{tabular}

Increase in Confidence with Sampling Intensity

\begin{tabular}{c|cccccc} 
& \multicolumn{7}{c}{ Sampling Intensity } \\
Variable Set & $\mathbf{1 2}$ & $\mathbf{2 5}$ & $\mathbf{5 0}$ & $\mathbf{1 0 0}$ & $\mathbf{2 0 0}$ & $\mathbf{3 0 0}$ \\
\hline \hline Dry & & 7.5 & 13.9 & 4.5 & 5.6 & 2.3 \\
Dry + Txt & & 3.8 & 8.5 & 4.7 & 6.6 & 2.9 \\
\hline Wet & & 11.7 & 8.5 & 6.0 & 4.4 & 1.3 \\
Wet + Txt & & 8.0 & 7.7 & 5.5 & 3.6 & 2.0 \\
\hline Wet \& Dry & & 7.3 & 10.7 & 2.8 & 2.4 & 1.5 \\
Wet \& Dry + Txt & & 14.4 & 6.4 & 6.8 & 3.2 & 1.6 \\
\hline
\end{tabular}


Table 3.7. Class-specific mean and standard error (SE) estimates of producer's and user's accuracies in percent by variable set. Parameters were estimated from the 10 model results for random forest classifier models for a sample size of 200. Ridge classes in white (Fig. 3.7), slough classes in grey (Fig. 3.8). Var. Set $=$ Variable set; Refl. $=$ Reflective bands; Txt. = Texture variables; $\mathbf{B i}=$ Bi-seasonal data Dry $=$ Dry season predicted class label; Wet $=$ Wet season predicted class label.

\begin{tabular}{|c|c|c|c|c|c|}
\hline \multirow{2}{*}{$\begin{array}{c}\text { Var. } \\
\text { Set }\end{array}$} & \multirow[t]{2}{*}{ Class Name } & \multicolumn{2}{|c|}{$\begin{array}{c}\text { Producer's } \\
\text { Accuracy }\end{array}$} & \multicolumn{2}{|c|}{$\begin{array}{c}\text { User's } \\
\text { Accuracy }\end{array}$} \\
\hline & & Mean & SE & Mean & SE \\
\hline \multirow{8}{*}{ 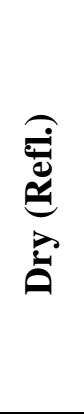 } & Submerged Aquatic & $96.5 \%$ & $0.5 \%$ & $98.6 \%$ & $0.3 \%$ \\
\hline & Submerged Aquatic / Periphyton / Floating Broadleaf & $82.7 \%$ & $0.4 \%$ & $72.4 \%$ & $1.4 \%$ \\
\hline & Floating Broadleaf / Submerged Aquatic & $89.6 \%$ & $0.9 \%$ & $93.6 \%$ & $0.8 \%$ \\
\hline & Floating Broadleaf Nymphaea & $85.2 \%$ & $1.4 \%$ & $78.7 \%$ & $1.0 \%$ \\
\hline & Graminoid Marsh / Emergent Broadleaf & $45.5 \%$ & $1.5 \%$ & $43.7 \%$ & $0.9 \%$ \\
\hline & Graminoid marsh Cladium & $62.7 \%$ & $1.2 \%$ & $73.1 \%$ & $0.3 \%$ \\
\hline & Shrub / Graminoid Marsh / Emergent Broadleaf & $65.3 \%$ & $1.1 \%$ & $84.6 \%$ & $0.7 \%$ \\
\hline & Shrub / Tree & $70.0 \%$ & $2.1 \%$ & $34.6 \%$ & $0.8 \%$ \\
\hline \multirow{8}{*}{ 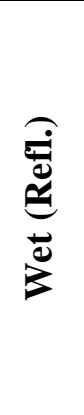 } & Submerged Aquatic & $98.8 \%$ & $0.8 \%$ & $68.8 \%$ & $1.4 \%$ \\
\hline & Submerged Aquatic / Periphyton / Floating Broadleaf & $92.0 \%$ & $0.7 \%$ & $99.0 \%$ & $0.1 \%$ \\
\hline & Floating Broadleaf / Submerged Aquatic & $84.4 \%$ & $1.0 \%$ & $88.8 \%$ & $0.8 \%$ \\
\hline & Floating Broadleaf Nymphaea & $86.8 \%$ & $0.8 \%$ & $88.6 \%$ & $0.9 \%$ \\
\hline & Graminoid Marsh / Emergent Broadleaf & $83.5 \%$ & $1.0 \%$ & $69.9 \%$ & $1.1 \%$ \\
\hline & Graminoid marsh Cladium & $91.2 \%$ & $0.5 \%$ & $87.2 \%$ & $0.4 \%$ \\
\hline & Shrub / Graminoid Marsh / Emergent Broadleaf & $45.0 \%$ & $1.3 \%$ & $59.1 \%$ & $1.4 \%$ \\
\hline & Shrub / Tree & $99.1 \%$ & $0.6 \%$ & $93.7 \%$ & $1.7 \%$ \\
\hline \multirow{8}{*}{ 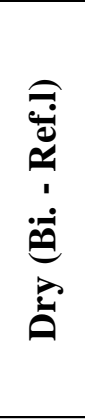 } & Submerged Aquatic & $97.7 \%$ & $0.4 \%$ & $98.0 \%$ & $0.2 \%$ \\
\hline & Submerged Aquatic / Periphyton / Floating Broadleaf & $92.9 \%$ & $0.4 \%$ & $91.6 \%$ & $0.4 \%$ \\
\hline & Floating Broadleaf / Submerged Aquatic & $89.3 \%$ & $0.4 \%$ & $97.0 \%$ & $0.4 \%$ \\
\hline & Floating Broadleaf Nymphaea & $92.8 \%$ & $0.6 \%$ & $81.9 \%$ & $0.3 \%$ \\
\hline & Graminoid Marsh / Emergent Broadleaf & $85.4 \%$ & $0.9 \%$ & $85.8 \%$ & $0.7 \%$ \\
\hline & Graminoid marsh Cladium & $94.4 \%$ & $0.6 \%$ & $93.4 \%$ & $0.5 \%$ \\
\hline & Shrub / Graminoid Marsh / Emergent Broadleaf & $86.0 \%$ & $1.3 \%$ & $96.5 \%$ & $0.7 \%$ \\
\hline & Shrub / Tree & $98.3 \%$ & $1.3 \%$ & $98.8 \%$ & $0.6 \%$ \\
\hline \multirow{8}{*}{ 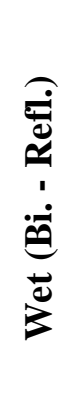 } & Submerged Aquatic & $100.0 \%$ & $0.0 \%$ & $73.3 \%$ & $1.6 \%$ \\
\hline & Submerged Aquatic / Periphyton / Floating Broadleaf & $95.7 \%$ & $0.3 \%$ & $98.3 \%$ & $0.1 \%$ \\
\hline & Floating Broadleaf / Submerged Aquatic & $93.7 \%$ & $0.3 \%$ & $83.9 \%$ & $0.7 \%$ \\
\hline & Floating Broadleaf Nymphaea & $88.5 \%$ & $0.8 \%$ & $91.3 \%$ & $0.9 \%$ \\
\hline & Graminoid Marsh / Emergent Broadleaf & $85.4 \%$ & $0.9 \%$ & $85.8 \%$ & $0.7 \%$ \\
\hline & Graminoid marsh Cladium & $94.4 \%$ & $0.6 \%$ & $93.4 \%$ & $0.5 \%$ \\
\hline & Shrub / Graminoid Marsh / Emergent Broadleaf & $86.0 \%$ & $1.3 \%$ & $96.5 \%$ & $0.7 \%$ \\
\hline & Shrub / Tree & $98.3 \%$ & $1.3 \%$ & $98.8 \%$ & $0.6 \%$ \\
\hline
\end{tabular}


Table 3.7. Continued.

\begin{tabular}{|c|c|c|c|c|c|}
\hline \multirow{2}{*}{$\begin{array}{l}\text { Var. } \\
\text { Set }\end{array}$} & \multirow[t]{2}{*}{ Class Name } & \multicolumn{2}{|c|}{$\begin{array}{c}\text { Producer's } \\
\text { Accuracy }\end{array}$} & \multicolumn{2}{|c|}{$\begin{array}{c}\text { User's } \\
\text { Accuracy }\end{array}$} \\
\hline & & Mean & SE & Mean & SE \\
\hline \multirow{8}{*}{ 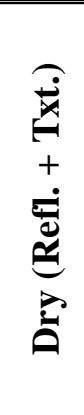 } & Submerged Aquatic & $95.9 \%$ & $0.8 \%$ & $98.9 \%$ & $0.3 \%$ \\
\hline & Submerged Aquatic / Periphyton / Floating Broadleaf & $83.2 \%$ & $0.6 \%$ & $69.8 \%$ & $0.8 \%$ \\
\hline & Floating Broadleaf / Submerged Aquatic & $91.2 \%$ & $0.9 \%$ & $93.0 \%$ & $0.9 \%$ \\
\hline & Floating Broadleaf Nymphaea & $87.3 \%$ & $1.5 \%$ & $77.2 \%$ & $1.6 \%$ \\
\hline & Graminoid Marsh / Emergent Broadleaf & $45.9 \%$ & $0.9 \%$ & $45.4 \%$ & $0.6 \%$ \\
\hline & Graminoid marsh Cladium & $59.8 \%$ & $1.1 \%$ & $74.0 \%$ & $0.6 \%$ \\
\hline & Shrub / Graminoid Marsh / Emergent Broadleaf & $65.9 \%$ & $1.5 \%$ & $83.0 \%$ & $0.7 \%$ \\
\hline & Shrub / Tree & $66.1 \%$ & $1.7 \%$ & $32.6 \%$ & $1.4 \%$ \\
\hline \multirow{8}{*}{ 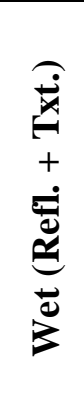 } & Submerged Aquatic & $100.0 \%$ & $0.0 \%$ & $66.5 \%$ & $0.7 \%$ \\
\hline & Submerged Aquatic / Periphyton / Floating Broadleaf & $91.3 \%$ & $0.5 \%$ & $99.2 \%$ & $0.2 \%$ \\
\hline & Floating Broadleaf / Submerged Aquatic & $82.3 \%$ & $0.9 \%$ & $88.2 \%$ & $0.9 \%$ \\
\hline & Floating Broadleaf Nymphaea & $86.5 \%$ & $1.2 \%$ & $85.8 \%$ & $1.0 \%$ \\
\hline & Graminoid Marsh / Emergent Broadleaf & $87.8 \%$ & $1.1 \%$ & $71.6 \%$ & $1.2 \%$ \\
\hline & Graminoid marsh Cladium & $90.3 \%$ & $1.0 \%$ & $88.1 \%$ & $0.6 \%$ \\
\hline & Shrub / Graminoid Marsh / Emergent Broadleaf & $47.1 \%$ & $1.5 \%$ & $59.8 \%$ & $2.4 \%$ \\
\hline & Shrub / Tree & $98.7 \%$ & $0.9 \%$ & $91.1 \%$ & $1.7 \%$ \\
\hline \multirow{8}{*}{ 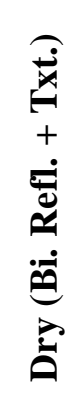 } & Submerged Aquatic & $97.2 \%$ & $0.3 \%$ & $98.4 \%$ & $0.3 \%$ \\
\hline & Submerged Aquatic / Periphyton / Floating Broadleaf & $91.9 \%$ & $0.3 \%$ & $93.3 \%$ & $0.3 \%$ \\
\hline & Floating Broadleaf / Submerged Aquatic & $91.8 \%$ & $0.6 \%$ & $94.4 \%$ & $0.7 \%$ \\
\hline & Floating Broadleaf Nymphaea & $90.9 \%$ & $1.0 \%$ & $81.9 \%$ & $1.0 \%$ \\
\hline & Graminoid Marsh / Emergent Broadleaf & $87.5 \%$ & $0.8 \%$ & $88.2 \%$ & $0.7 \%$ \\
\hline & Graminoid marsh Cladium & $95.5 \%$ & $0.4 \%$ & $93.6 \%$ & $0.4 \%$ \\
\hline & Shrub / Graminoid Marsh / Emergent Broadleaf & $87.8 \%$ & $0.5 \%$ & $96.4 \%$ & $0.4 \%$ \\
\hline & Shrub / Tree & $99.6 \%$ & $0.4 \%$ & $98.0 \%$ & $0.9 \%$ \\
\hline \multirow{8}{*}{ 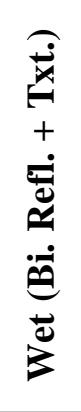 } & Submerged Aquatic & $100.0 \%$ & $0.0 \%$ & $75.0 \%$ & $1.1 \%$ \\
\hline & Submerged Aquatic / Periphyton / Floating Broadleaf & $95.6 \%$ & $0.3 \%$ & $98.0 \%$ & $0.2 \%$ \\
\hline & Floating Broadleaf / Submerged Aquatic & $91.8 \%$ & $0.4 \%$ & $87.7 \%$ & $0.6 \%$ \\
\hline & Floating Broadleaf Nymphaea & $89.3 \%$ & $1.0 \%$ & $88.4 \%$ & $0.9 \%$ \\
\hline & Graminoid Marsh / Emergent Broadleaf & $87.5 \%$ & $0.8 \%$ & $88.2 \%$ & $0.7 \%$ \\
\hline & Graminoid marsh Cladium & $95.5 \%$ & $0.4 \%$ & $93.6 \%$ & $0.4 \%$ \\
\hline & Shrub / Graminoid Marsh / Emergent Broadleaf & $87.8 \%$ & $0.5 \%$ & $96.4 \%$ & $0.4 \%$ \\
\hline & Shrub / Tree & $99.6 \%$ & $0.4 \%$ & $98.0 \%$ & $0.9 \%$ \\
\hline
\end{tabular}


Table 3.8. Class-specific user's accuracy differences by variable set. Upper and lower 95\% confidence estimates and median difference estimates (Hodges-Lehmann estimator). Ridge classes in white (Fig. 3.7), slough classes in grey (Fig. 3.8). Var. = Variable; Est. Diff. = Estimated Difference; Conf. $=$ Confidence Interval; Sign. $=$ Significance, where $-,{ }^{*}, * *$ and $* * *$ indicate not significant, significant at alpha $<0.05,0.01$ and 0.001 , respectively. $\mathbf{T x t} .=$ Texture; Refl. $=$ Reflective bands; Txt. $=$ Texture variables; $\mathbf{B} \mathbf{i}=$ Bi-seasonal data; Dry $=$ Dry season predicted class label; Wet $=$ Wet season predicted class label.

\begin{tabular}{|c|c|c|c|c|c|}
\hline $\begin{array}{l}\text { Var. } \\
\text { Set }\end{array}$ & Class Name & Est. Diff. & $95 \%$ & Conf. & Sign. \\
\hline \multirow{8}{*}{ 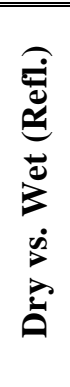 } & Submerged Aquatic & $30.4 \%$ & $27.6 \%$ & $32.0 \%$ & $* * *$ \\
\hline & Submerged Aquatic / Periphyton / Floating Broadleaf & $-26.0 \%$ & $-30.8 \%$ & $-24.9 \%$ & $* * *$ \\
\hline & Floating Broadleaf / Submerged Aquatic & $4.8 \%$ & $2.3 \%$ & $7.3 \%$ & $* * *$ \\
\hline & Floating Broadleaf Nymphaea & $-9.6 \%$ & $-13.1 \%$ & $-7.1 \%$ & $* * *$ \\
\hline & Graminoid Marsh / Emergent Broadleaf & $-26.2 \%$ & $-29.2 \%$ & $-23.4 \%$ & $* * *$ \\
\hline & Graminoid marsh Cladium & $-14.1 \%$ & $-15.4 \%$ & $-13.1 \%$ & $* * *$ \\
\hline & Shrub / Graminoid Marsh / Emergent Broadleaf & $25.8 \%$ & $21.6 \%$ & $29.1 \%$ & $* * *$ \\
\hline & Shrub / Tree & $-59.2 \%$ & $-63.9 \%$ & $-54.5 \%$ & $* * *$ \\
\hline \multirow{8}{*}{ 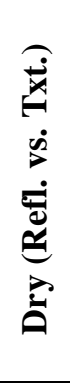 } & Submerged Aquatic & $-0.3 \%$ & $-1.0 \%$ & $0.3 \%$ & - \\
\hline & Submerged Aquatic / Periphyton / Floating Broadleaf & $2.8 \%$ & $-1.5 \%$ & $6.5 \%$ & - \\
\hline & Floating Broadleaf / Submerged Aquatic & $0.4 \%$ & $-2.2 \%$ & $3.2 \%$ & - \\
\hline & Floating Broadleaf Nymphaea & $2.4 \%$ & $-2.4 \%$ & $5.9 \%$ & - \\
\hline & Graminoid Marsh / Emergent Broadleaf & $-1.9 \%$ & $-3.7 \%$ & $-0.2 \%$ & $*$ \\
\hline & Graminoid marsh Cladium & $-0.8 \%$ & $-2.4 \%$ & $0.3 \%$ & - \\
\hline & Shrub / Graminoid Marsh / Emergent Broadleaf & $1.7 \%$ & $-0.7 \%$ & $4.3 \%$ & - \\
\hline & Shrub / Tree & $1.8 \%$ & $-1.4 \%$ & $5.3 \%$ & - \\
\hline \multirow{8}{*}{ 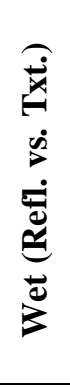 } & Submerged Aquatic & $2.6 \%$ & $0.0 \%$ & $5.4 \%$ & - \\
\hline & Submerged Aquatic / Periphyton / Floating Broadleaf & $-0.1 \%$ & $-0.6 \%$ & $0.4 \%$ & - \\
\hline & Floating Broadleaf / Submerged Aquatic & $0.3 \%$ & $-2.3 \%$ & $3.1 \%$ & - \\
\hline & Floating Broadleaf Nymphaea & $3.2 \%$ & $-0.3 \%$ & $6.2 \%$ & - \\
\hline & Graminoid Marsh / Emergent Broadleaf & $-2.2 \%$ & $-5.7 \%$ & $1.4 \%$ & - \\
\hline & Graminoid marsh Cladium & $-1.0 \%$ & $-2.7 \%$ & $0.9 \%$ & - \\
\hline & Shrub / Graminoid Marsh / Emergent Broadleaf & $-1.1 \%$ & $-7.5 \%$ & $5.9 \%$ & - \\
\hline & Shrub / Tree & $3.4 \%$ & $-3.3 \%$ & $8.0 \%$ & - \\
\hline
\end{tabular}


Table 3.8. Continued.

\begin{tabular}{|c|c|c|c|c|c|}
\hline $\begin{array}{l}\text { Var. } \\
\text { Set }\end{array}$ & Class Name & Est. Diff. & $95 \%$ & Conf. & Sign. \\
\hline \multirow{8}{*}{ 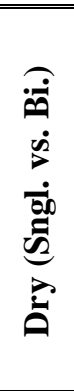 } & Submerged Aquatic & $0.6 \%$ & $0.0 \%$ & $1.3 \%$ & - \\
\hline & Submerged Aquatic / Periphyton / Floating Broadleaf & $-18.8 \%$ & $-23.5 \%$ & $-16.4 \%$ & $* * *$ \\
\hline & Floating Broadleaf / Submerged Aquatic & $-3.5 \%$ & $-5.8 \%$ & $-1.1 \%$ & $* *$ \\
\hline & Floating Broadleaf Nymphaea & $-3.1 \%$ & $-5.9 \%$ & $-0.1 \%$ & $*$ \\
\hline & Graminoid Marsh / Emergent Broadleaf & $-42.3 \%$ & $-44.4 \%$ & $-39.9 \%$ & $* * *$ \\
\hline & Graminoid marsh Cladium & $-20.6 \%$ & $-21.9 \%$ & $-18.6 \%$ & $* * *$ \\
\hline & Shrub / Graminoid Marsh / Emergent Broadleaf & $-11.8 \%$ & $-13.7 \%$ & $-10.0 \%$ & $* * *$ \\
\hline & Shrub / Tree & $-64.1 \%$ & $-66.7 \%$ & $-62.2 \%$ & $* * *$ \\
\hline \multirow{8}{*}{ 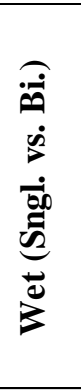 } & Submerged Aquatic & $-4.4 \%$ & $-9.3 \%$ & $0.0 \%$ & $*$ \\
\hline & Submerged Aquatic / Periphyton / Floating Broadleaf & $0.7 \%$ & $0.3 \%$ & $1.0 \%$ & $* * *$ \\
\hline & Floating Broadleaf / Submerged Aquatic & $4.7 \%$ & $2.4 \%$ & $7.0 \%$ & $* * *$ \\
\hline & Floating Broadleaf Nymphaea & $-2.9 \%$ & $-5.4 \%$ & $-0.1 \%$ & $*$ \\
\hline & Graminoid Marsh / Emergent Broadleaf & $-16.2 \%$ & $-19.1 \%$ & $-13.1 \%$ & $* * *$ \\
\hline & Graminoid marsh Cladium & $-6.2 \%$ & $-7.9 \%$ & $-4.6 \%$ & $* * *$ \\
\hline & Shrub / Graminoid Marsh / Emergent Broadleaf & $-37.9 \%$ & $-41.2 \%$ & $-33.6 \%$ & $* * *$ \\
\hline & Shrub / Tree & $-4.3 \%$ & $-8.0 \%$ & $0.0 \%$ & $*$ \\
\hline \multirow{8}{*}{ 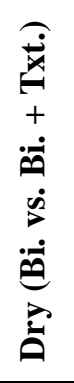 } & Submerged Aquatic & $-0.3 \%$ & $-1.2 \%$ & $0.3 \%$ & - \\
\hline & Submerged Aquatic / Periphyton / Floating Broadleaf & $-1.7 \%$ & $-2.7 \%$ & $-0.5 \%$ & $* *$ \\
\hline & Floating Broadleaf / Submerged Aquatic & $2.3 \%$ & $1.6 \%$ & $3.2 \%$ & $* *$ \\
\hline & Floating Broadleaf Nymphaea & $0.1 \%$ & $-2.0 \%$ & $2.4 \%$ & - \\
\hline & Graminoid Marsh / Emergent Broadleaf & $-2.2 \%$ & $-4.5 \%$ & $-0.2 \%$ & $*$ \\
\hline & Graminoid marsh Cladium & $0.1 \%$ & $-1.8 \%$ & $1.4 \%$ & - \\
\hline & Shrub / Graminoid Marsh / Emergent Broadleaf & $0.0 \%$ & $-1.1 \%$ & $1.7 \%$ & - \\
\hline & Shrub / Tree & $0.0 \%$ & $0.0 \%$ & $4.2 \%$ & - \\
\hline \multirow{8}{*}{ 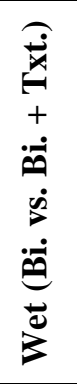 } & Submerged Aquatic & $-3.1 \%$ & $-6.4 \%$ & $3.4 \%$ & - \\
\hline & Submerged Aquatic / Periphyton / Floating Broadleaf & $0.4 \%$ & $-0.1 \%$ & $0.8 \%$ & - \\
\hline & Floating Broadleaf / Submerged Aquatic & $-3.7 \%$ & $-5.6 \%$ & $-1.8 \%$ & $* * *$ \\
\hline & Floating Broadleaf Nymphaea & $3.1 \%$ & $0.4 \%$ & $5.7 \%$ & $*$ \\
\hline & Graminoid Marsh / Emergent Broadleaf & $-2.2 \%$ & $-4.5 \%$ & $-0.2 \%$ & $*$ \\
\hline & Graminoid marsh Cladium & $0.1 \%$ & $-1.8 \%$ & $1.4 \%$ & - \\
\hline & Shrub / Graminoid Marsh / Emergent Broadleaf & $0.0 \%$ & $-1.1 \%$ & $1.7 \%$ & - \\
\hline & Shrub / Tree & $0.0 \%$ & $0.0 \%$ & $4.2 \%$ & - \\
\hline
\end{tabular}


Table 3.9. Class-specific producer's accuracy differences by variable set. Upper and lower 95\% confidence estimates and median difference estimates (Hodges-Lehmann estimator). Ridge classes in white (Fig. 3.7), slough classes in grey (Fig. 3.8). Var. = Variable; Est. Diff. = Estimated Difference; Conf. $=$ Confidence Interval; Sign. $=$ Significance, where $-,{ }^{*}, * *$ and $* * *$ indicate not significant, significant at alpha $<0.05,0.01$ and 0.001 , respectively. Txt. = Texture; Refl. $=$ Reflective bands; Txt. $=$ Texture variables; $\mathbf{B} \mathbf{i}=$ Bi-seasonal data; Dry $=$ Dry season predicted class label; Wet $=$ Wet season predicted class label.

\begin{tabular}{|c|c|c|c|c|c|}
\hline $\begin{array}{l}\text { Var. } \\
\text { Set }\end{array}$ & Class Name & Est. Diff. & $95 \%$ & Conf. & Sign. \\
\hline \multirow{8}{*}{ 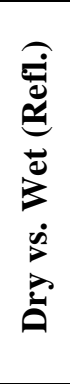 } & Submerged Aquatic & $-2.9 \%$ & $-4.2 \%$ & $-1.3 \%$ & * \\
\hline & Submerged Aquatic / Periphyton / Floating Broadleaf & $-9.5 \%$ & $-11.3 \%$ & $-7.4 \%$ & $* * *$ \\
\hline & Floating Broadleaf / Submerged Aquatic & $5.0 \%$ & $1.9 \%$ & $8.4 \%$ & $* *$ \\
\hline & Floating Broadleaf Nymphaea & $-1.7 \%$ & $-5.7 \%$ & $2.4 \%$ & - \\
\hline & Graminoid Marsh / Emergent Broadleaf & $-37.8 \%$ & $-42.6 \%$ & $-33.9 \%$ & $* * *$ \\
\hline & Graminoid marsh Cladium & $-28.8 \%$ & $-30.6 \%$ & $-25.6 \%$ & $* * *$ \\
\hline & Shrub / Graminoid Marsh / Emergent Broadleaf & $19.8 \%$ & $16.3 \%$ & $24.8 \%$ & $* * *$ \\
\hline & Shrub / Tree & $-30.4 \%$ & $-34.8 \%$ & $-26.1 \%$ & $* * *$ \\
\hline \multirow{8}{*}{ 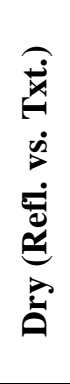 } & Submerged Aquatic & $0.6 \%$ & $-1.6 \%$ & $2.9 \%$ & - \\
\hline & Submerged Aquatic / Periphyton / Floating Broadleaf & $-0.4 \%$ & $-1.8 \%$ & $1.1 \%$ & - \\
\hline & Floating Broadleaf / Submerged Aquatic & $-1.6 \%$ & $-4.4 \%$ & $1.4 \%$ & - \\
\hline & Floating Broadleaf Nymphaea & $-2.4 \%$ & $-6.6 \%$ & $2.2 \%$ & - \\
\hline & Graminoid Marsh / Emergent Broadleaf & $0.3 \%$ & $-4.5 \%$ & $4.2 \%$ & - \\
\hline & Graminoid marsh Cladium & $2.9 \%$ & $-0.8 \%$ & $6.9 \%$ & - \\
\hline & Shrub / Graminoid Marsh / Emergent Broadleaf & $-0.8 \%$ & $-5.4 \%$ & $3.1 \%$ & - \\
\hline & Shrub / Tree & $4.4 \%$ & $-4.3 \%$ & $8.7 \%$ & - \\
\hline \multirow{8}{*}{ 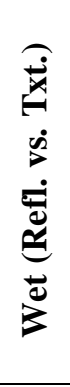 } & Submerged Aquatic & $0.0 \%$ & $0.0 \%$ & $0.0 \%$ & - \\
\hline & Submerged Aquatic / Periphyton / Floating Broadleaf & $1.0 \%$ & $-1.3 \%$ & $3.0 \%$ & - \\
\hline & Floating Broadleaf / Submerged Aquatic & $2.3 \%$ & $-1.1 \%$ & $5.3 \%$ & - \\
\hline & Floating Broadleaf Nymphaea & $0.1 \%$ & $-3.1 \%$ & $3.6 \%$ & - \\
\hline & Graminoid Marsh / Emergent Broadleaf & $-4.2 \%$ & $-7.3 \%$ & $-1.1 \%$ & $*$ \\
\hline & Graminoid marsh Cladium & $0.2 \%$ & $-1.7 \%$ & $4.5 \%$ & - \\
\hline & Shrub / Graminoid Marsh / Emergent Broadleaf & $-1.6 \%$ & $-7.0 \%$ & $1.6 \%$ & - \\
\hline & Shrub / Tree & $0.0 \%$ & $0.0 \%$ & $0.0 \%$ & - \\
\hline
\end{tabular}


Table 3.9. Continued.

\begin{tabular}{|c|c|c|c|c|c|}
\hline $\begin{array}{l}\text { Var. } \\
\text { Set }\end{array}$ & Class Name & Est. Diff. & $95 \%$ & Conf. & Sign. \\
\hline \multirow{8}{*}{ 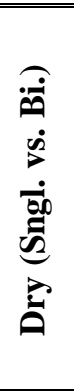 } & Submerged Aquatic & $-1.3 \%$ & $-2.6 \%$ & $0.3 \%$ & - \\
\hline & Submerged Aquatic / Periphyton / Floating Broadleaf & $-10.5 \%$ & $-11.5 \%$ & $-8.9 \%$ & $* * *$ \\
\hline & Floating Broadleaf / Submerged Aquatic & $-0.1 \%$ & $-2.0 \%$ & $3.0 \%$ & - \\
\hline & Floating Broadleaf Nymphaea & $-7.2 \%$ & $-11.1 \%$ & $-3.9 \%$ & $* * *$ \\
\hline & Graminoid Marsh / Emergent Broadleaf & $-39.5 \%$ & $-44.0 \%$ & $-35.3 \%$ & $* * *$ \\
\hline & Graminoid marsh Cladium & $-31.8 \%$ & $-34.2 \%$ & $-28.7 \%$ & $* * *$ \\
\hline & Shrub / Graminoid Marsh / Emergent Broadleaf & $-20.9 \%$ & $-24.8 \%$ & $-17.1 \%$ & $* * *$ \\
\hline & Shrub / Tree & $-30.4 \%$ & $-34.8 \%$ & $-21.7 \%$ & $* * *$ \\
\hline \multirow{8}{*}{ 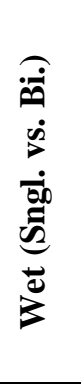 } & Submerged Aquatic & $0.0 \%$ & $0.0 \%$ & $0.0 \%$ & - \\
\hline & Submerged Aquatic / Periphyton / Floating Broadleaf & $-3.2 \%$ & $-5.4 \%$ & $-1.7 \%$ & $* * *$ \\
\hline & Floating Broadleaf / Submerged Aquatic & $-9.8 \%$ & $-11.7 \%$ & $-6.4 \%$ & $* * *$ \\
\hline & Floating Broadleaf Nymphaea & $-1.5 \%$ & $-4.1 \%$ & $1.0 \%$ & - \\
\hline & Graminoid Marsh / Emergent Broadleaf & $-2.0 \%$ & $-5.0 \%$ & $1.4 \%$ & - \\
\hline & Graminoid marsh Cladium & $-3.0 \%$ & $-5.0 \%$ & $-1.9 \%$ & $* *$ \\
\hline & Shrub / Graminoid Marsh / Emergent Broadleaf & $-41.1 \%$ & $-45.7 \%$ & $-38.0 \%$ & $* * *$ \\
\hline & Shrub / Tree & $0.0 \%$ & $0.0 \%$ & $0.0 \%$ & - \\
\hline \multirow{8}{*}{$\begin{array}{l}\stackrel{\dot{\vec{x}}}{\vec{H}} \\
+ \\
\dot{\theta} \\
\dot{\vec{n}} \\
\dot{\vec{\theta}} \\
\dot{\vec{\theta}} \\
\overrightarrow{\dot{\theta}}\end{array}$} & Submerged Aquatic & $0.6 \%$ & $0.0 \%$ & $1.6 \%$ & - \\
\hline & Submerged Aquatic / Periphyton / Floating Broadleaf & $1.1 \%$ & $0.0 \%$ & $2.1 \%$ & $*$ \\
\hline & Floating Broadleaf / Submerged Aquatic & $-2.4 \%$ & $-4.1 \%$ & $-0.7 \%$ & $*$ \\
\hline & Floating Broadleaf Nymphaea & $2.3 \%$ & $-1.1 \%$ & $5.0 \%$ & - \\
\hline & Graminoid Marsh / Emergent Broadleaf & $-1.8 \%$ & $-4.8 \%$ & $0.8 \%$ & - \\
\hline & Graminoid marsh Cladium & $-1.1 \%$ & $-2.8 \%$ & $0.5 \%$ & - \\
\hline & Shrub / Graminoid Marsh / Emergent Broadleaf & $-1.5 \%$ & $-3.9 \%$ & $1.5 \%$ & - \\
\hline & Shrub / Tree & $0.0 \%$ & $0.0 \%$ & $0.0 \%$ & - \\
\hline \multirow{8}{*}{ 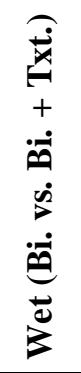 } & Submerged Aquatic & $0.0 \%$ & $0.0 \%$ & $0.0 \%$ & - \\
\hline & Submerged Aquatic / Periphyton / Floating Broadleaf & $0.1 \%$ & $-0.7 \%$ & $0.7 \%$ & - \\
\hline & Floating Broadleaf / Submerged Aquatic & $1.9 \%$ & $0.7 \%$ & $3.0 \%$ & $* *$ \\
\hline & Floating Broadleaf Nymphaea & $-1.0 \%$ & $-3.6 \%$ & $2.0 \%$ & - \\
\hline & Graminoid Marsh / Emergent Broadleaf & $-1.8 \%$ & $-4.8 \%$ & $0.8 \%$ & - \\
\hline & Graminoid marsh Cladium & $-1.1 \%$ & $-2.8 \%$ & $0.5 \%$ & - \\
\hline & Shrub / Graminoid Marsh / Emergent Broadleaf & $-1.5 \%$ & $-3.9 \%$ & $1.5 \%$ & - \\
\hline & Shrub / Tree & $0.0 \%$ & $0.0 \%$ & $0.0 \%$ & - \\
\hline
\end{tabular}




\section{FIGURES}
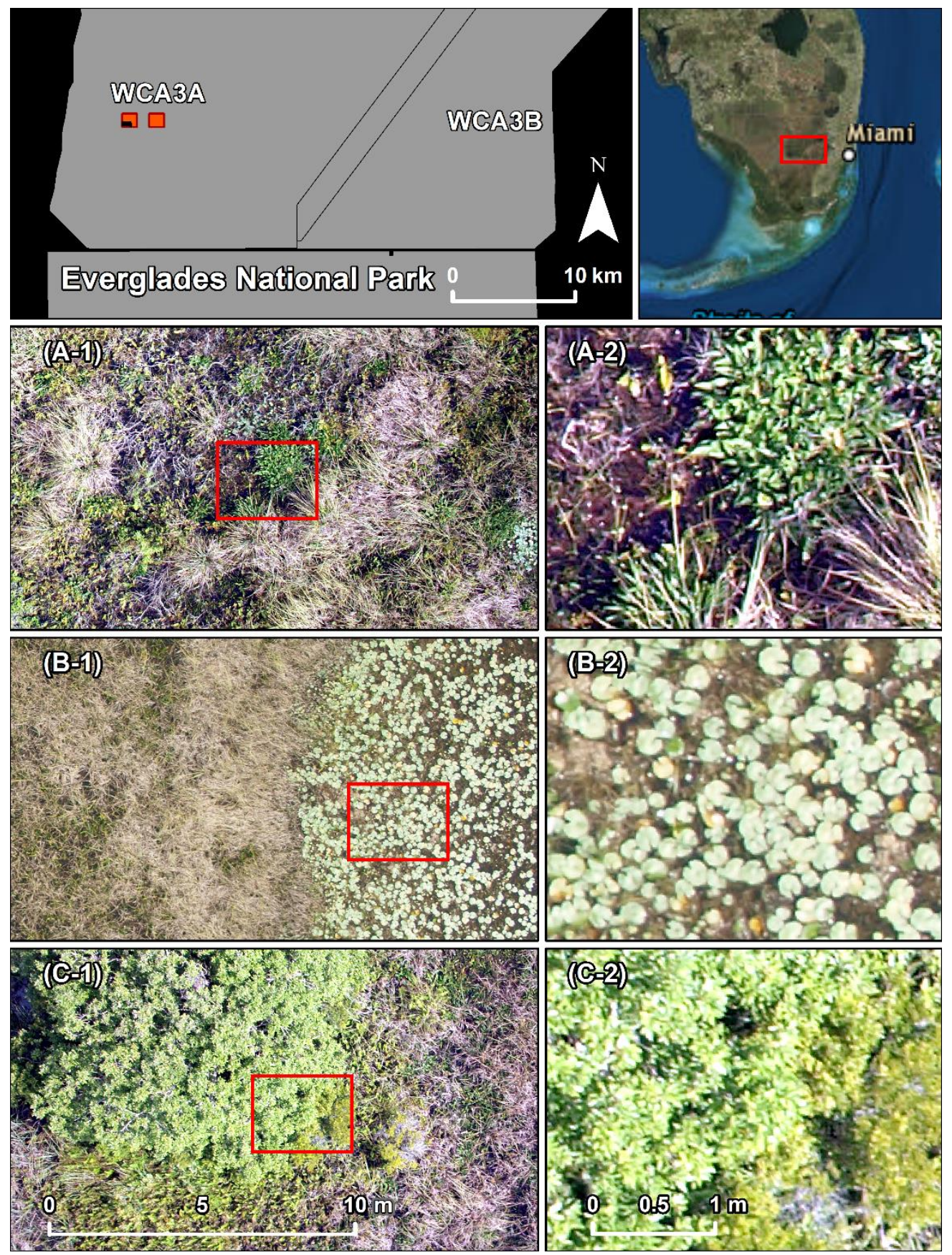

Figure 3.1. Study area: Two $1 \mathrm{~km}^{2}$ areas (red, West and East) located in southern WCA3A (top left, overview top right) and three examples of ridge and slough vegetation types. (A1 \& A2) Graminoid \& Broadleaf Marsh, (B1 \& B2) Nymphaea odorata, (C1 \& C2) Shrub \& Tree. Aerial photography ( $1 \mathrm{~cm}$ resolution) was acquired by a fixed-wing Unmanned Aerial System in 2012. 

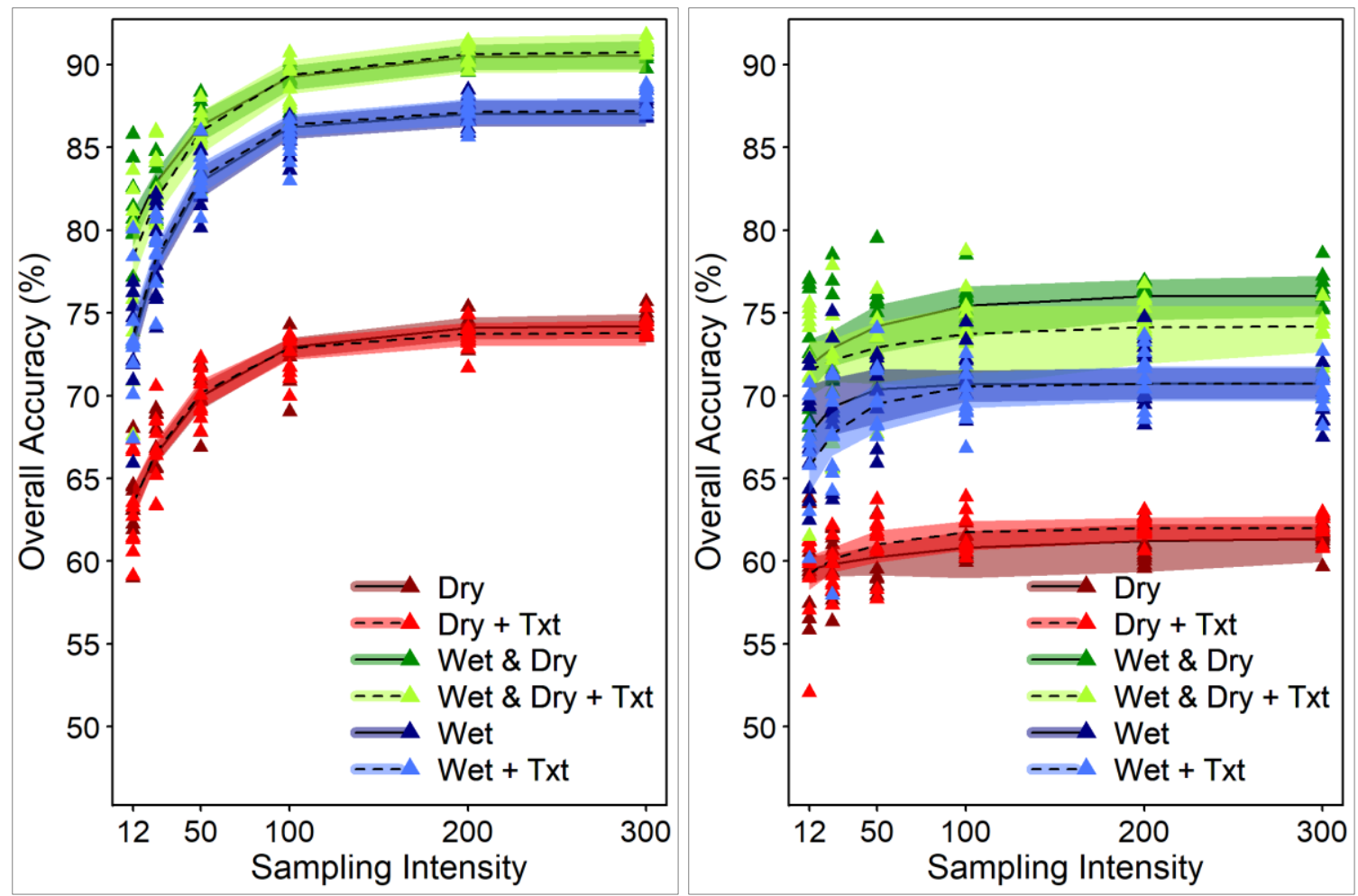

Figure 3.2. Overall accuracy (\%) by sampling intensity and variable set. Diminished returns regression model for random forest classifier (left) and naïve Bayes classifier (right); shading shows $95 \%$ confidence intervals of model mean using Monte Carlo error propagation model for non-linear models. 


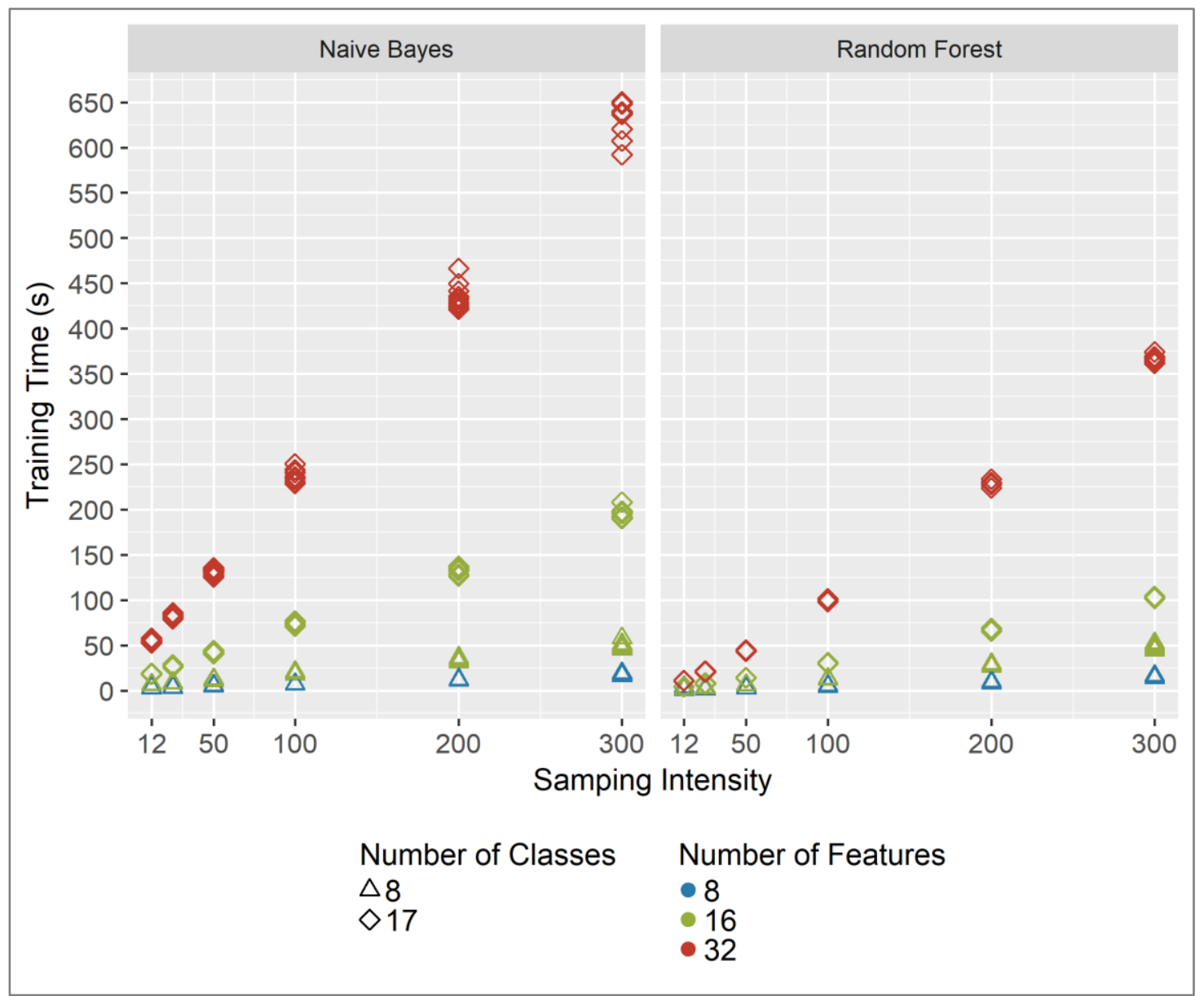

Figure 3.3. Training time in seconds as a function of sampling intensity, number of features and number of classes for Naïve Bayes and Random Forest classifiers. 


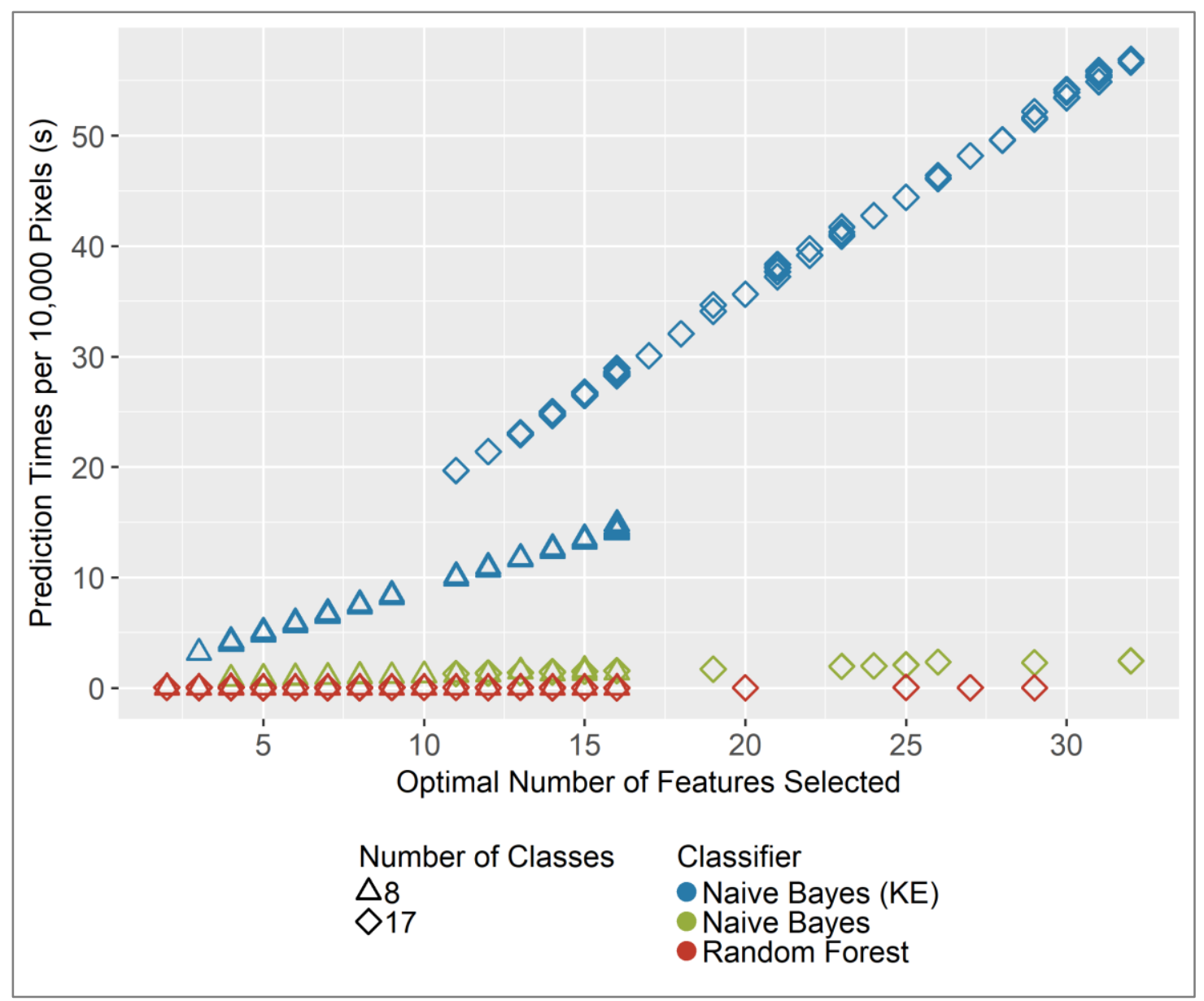

Figure 3.4. Prediction times for Naïve Bayes and Random Forest classifiers as a function of optimal number of selected features and number of classes. Naïve Bayes classifier was divided into tuned parameter with and without Kernel Estimator (KE). 


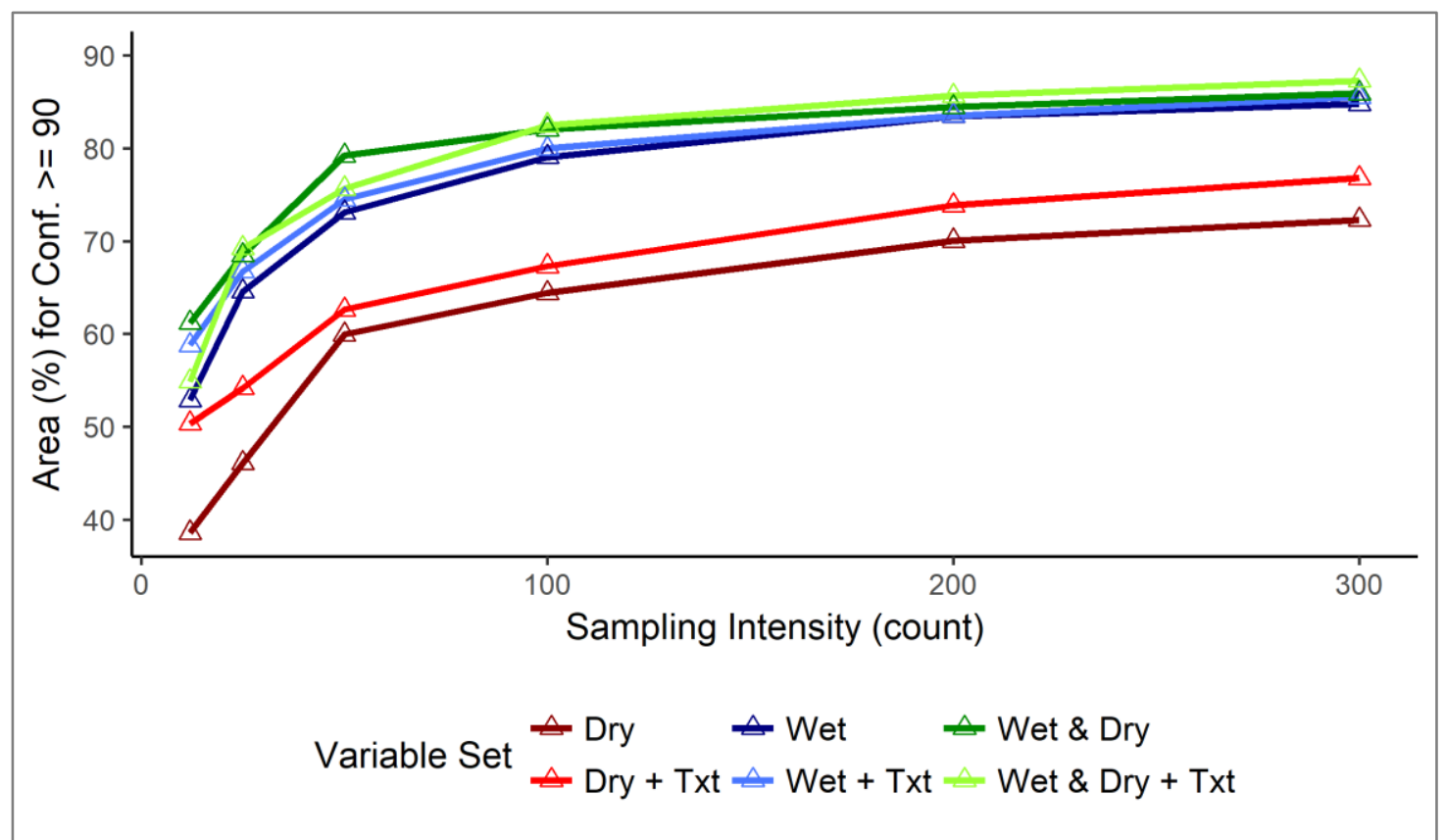

Figure 3.5. Percentage of map area for which location-specific confidence $\geq 90 \%(N=10)$ (Tbl. 3.6); Txt $=$ Texture. 


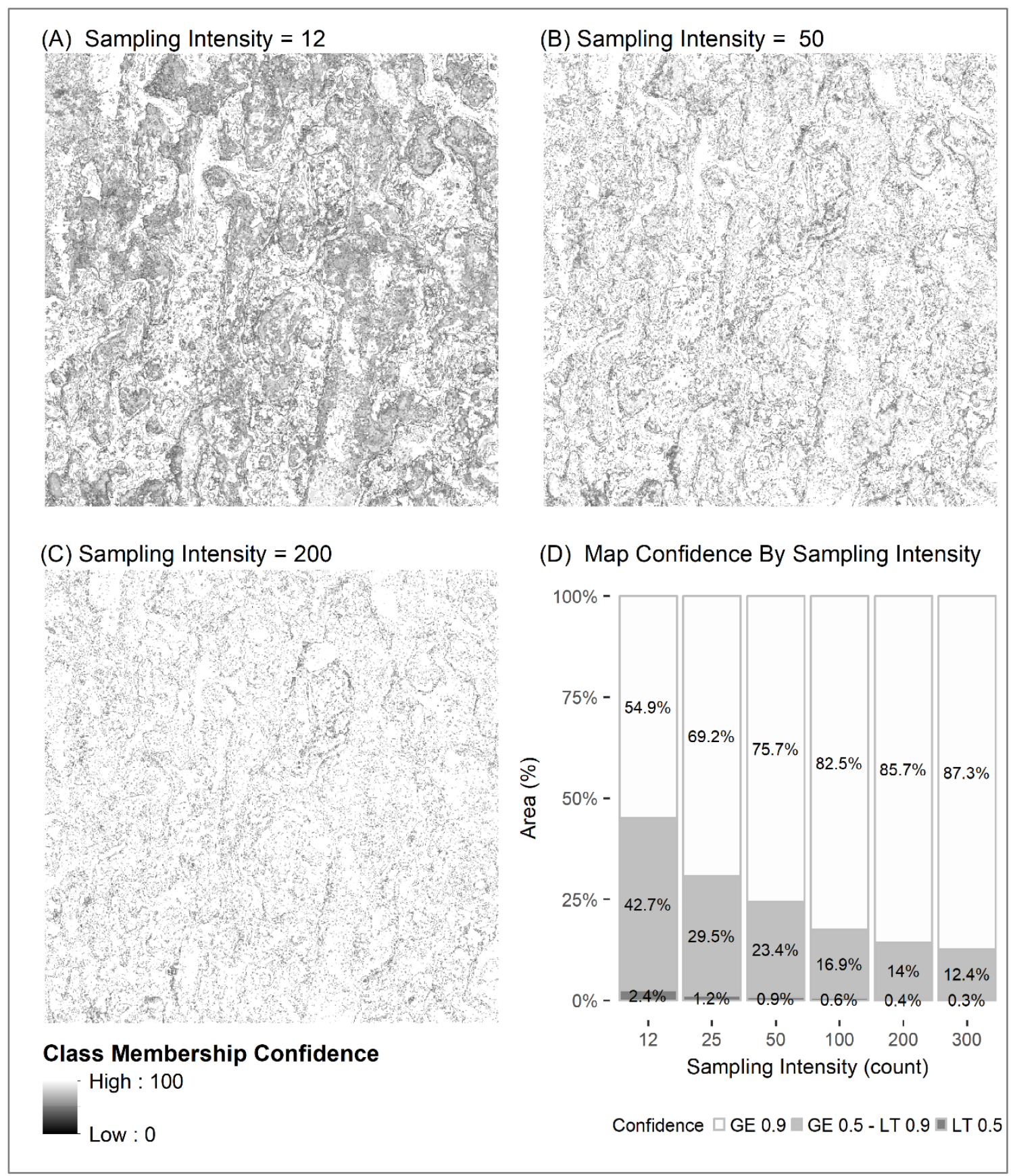

Figure 3.6. Location-specific class membership confidence $(N=10)$ by sampling intensity for sub-region East (Fig. 3.1, top left). (A) Sampling intensity = 12; (B) Sampling intensity = 50; (C) Sampling intensity $=200$. (D) Cumulative distribution of class confidence aggregated across full region of interest (Fig. 3.1., top left, East and West). For confidence brackets: GE = Greater Equal; LT $=$ Less Than. Numbers inside bars are percent of total area for each confidence bracket; note very low percentage for LT 0.5 


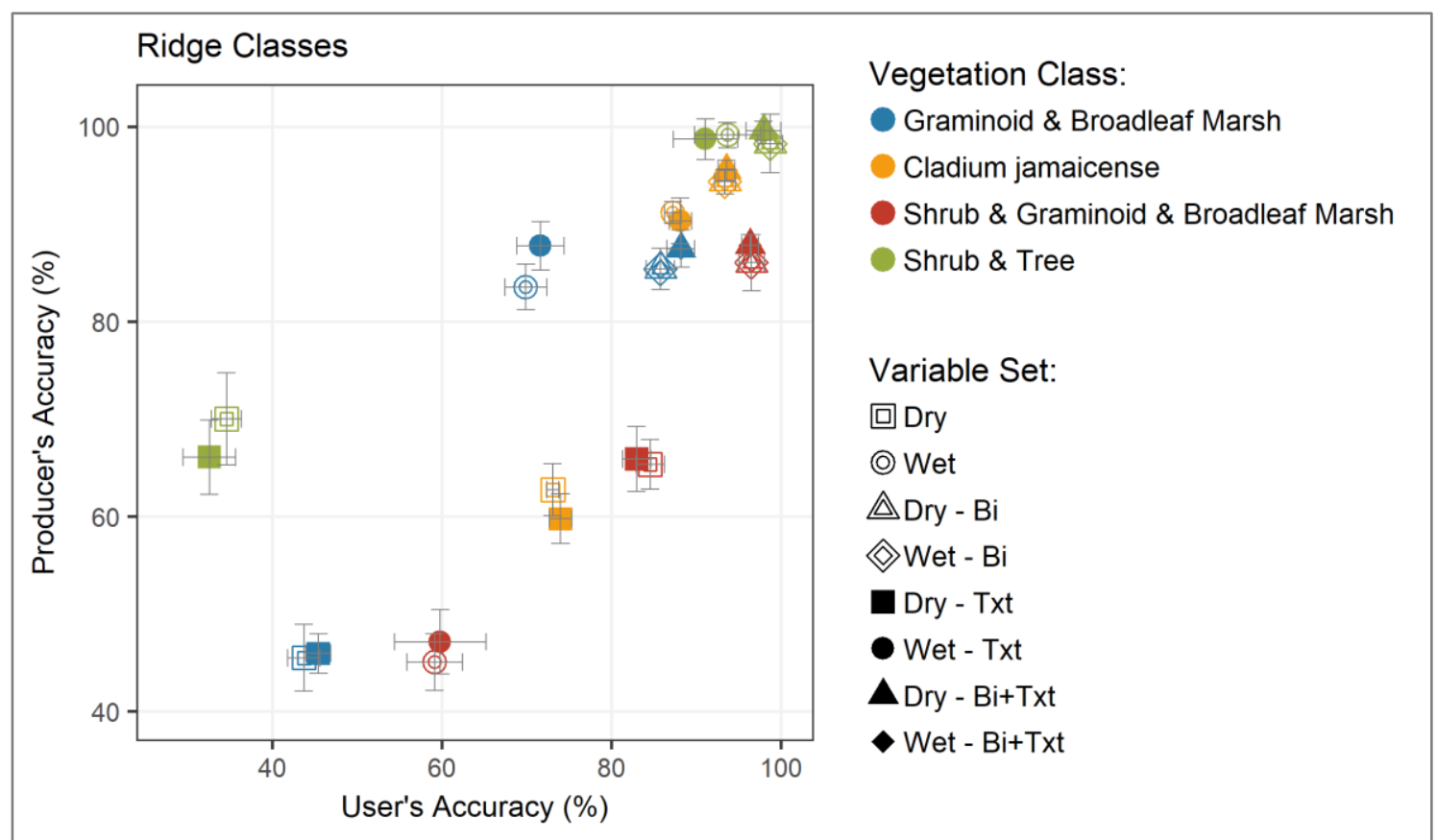

Figure 3.7. Class-specific user's and producer's accuracies and $95 \%$ confidence intervals for ridge classes; $\mathbf{B i}=$ Biseasonal; $\mathbf{T x t}=$ Texture.

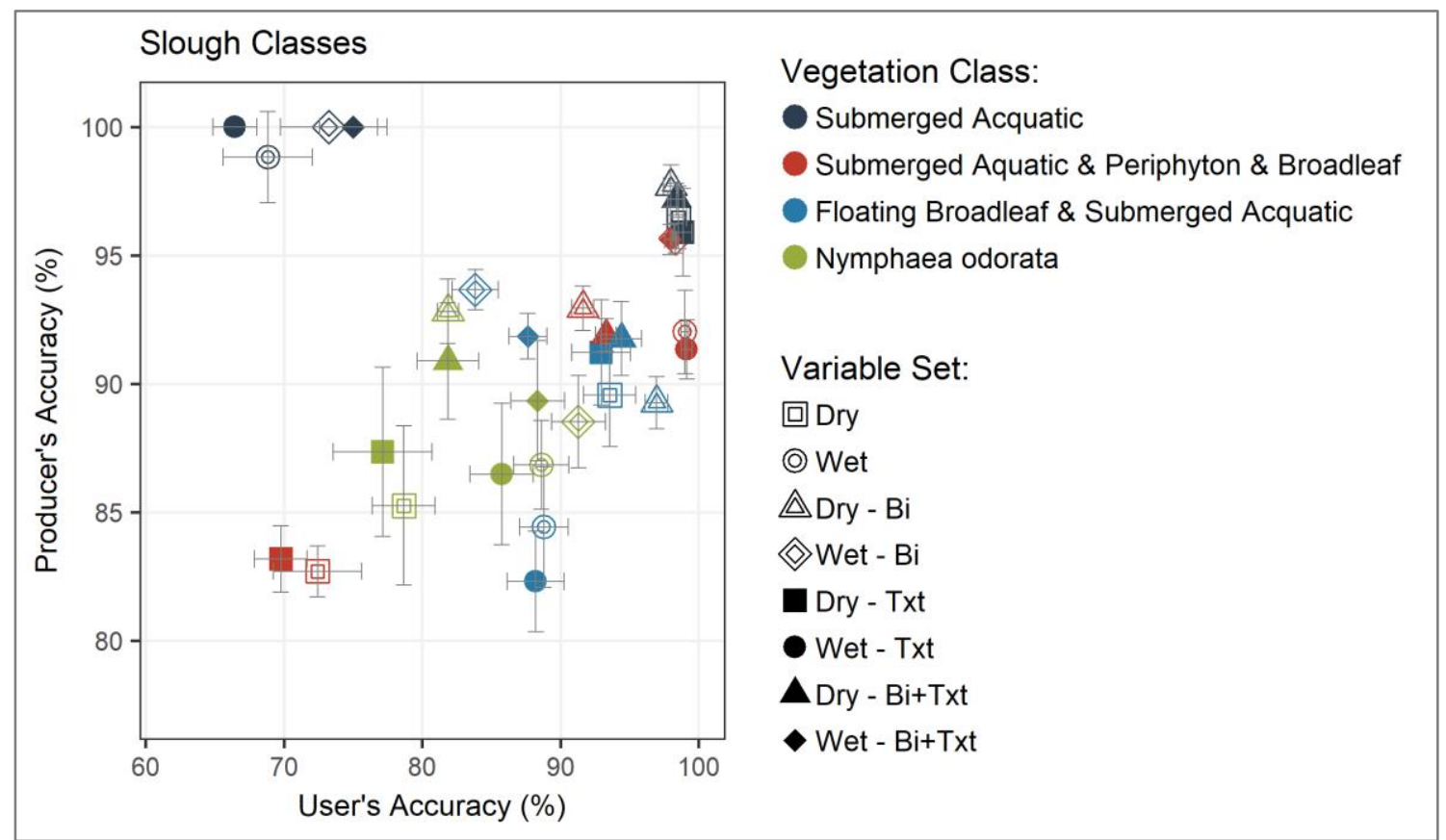

Figure 3.8. Class-specific user's and producer's accuracies and $95 \%$ confidence intervals for slough classes; $\mathbf{B i}=$ Biseasonal; $\mathbf{T x t}=$ Texture. 


\section{REFERENCES}

Atkinson, P. M., and A. R. L. Tatnall. 1997. "Introduction Neural Networks in Remote Sensing.” International Journal of Remote Sensing 18 (4):699-709. https://doi.org/10.1080/014311697218700.

Baty, F., C. Ritz, S. Charles, M. Brutsche, J. Flandrois, and M. Delignette-Muller. 2015. "A Toolbox for Nonlinear Regression in R: The Package Nlstools." Journal of Statistical Software 66 (5):1-21.

Belgiu, M., and L. Drăguţ. 2016. "Random Forest in Remote Sensing: A Review of Applications and Future Directions." ISPRS Journal of Photogrammetry and Remote Sensing 114 (April):24-31. https://doi.org/10.1016/j.isprsjprs.2016.01.011.

Bivand, R., T. Keitt, and B. Rowlingson. 2013. "Rgdal: Bindings for the Geospatial Data Abstraction Library". R package version 1.3. http://cran.rproject.org/package $=$ rgdal.

Boser, B. E., I. M. Guyon, and V. N. Vapnik. 1992. "A Training Algorithm for Optimal Margin Classifiers." In Proceedings of the Fifth Annual Workshop on Computational Learning Theory, 144-52. COLT' 92. New York, NY, USA: ACM. https://doi.org/10.1145/130385.130401.

Bovolo, F., G. Camps-Valls, and L. Bruzzone. 2010. "A Support Vector Domain Method for Change Detection in Multitemporal Images." Pattern Recognition Letters 31 (10):1148-54. https://doi.org/http://dx.doi.org/10.1016/j.patrec.2009.07.002.

Breiman, L. 2001. "Random Forests.” Machine Learning 45 (1):5-32. https://doi.org/10.1023/a:1010933404324.

Brown de Colstoun, E. C., and C. L. Walthall. 2006. "Improving Global Scale Land Cover Classifications with Multi-Directional POLDER Data and a Decision Tree Classifier." Remote Sensing of Environment 100 (4):474-85.

Congalton, R. G., and K. Green. 1999. Assessing the Accuracy of Remotely Sensed Data: Principles and Practices. Boca Raton, Florida 33431: Lewis Publishers.

Cortes, C., and V. Vapnik. 1995. "Support-Vector Networks.” Machine Learning 20 (3):273-97. https://doi.org/10.1007/bf00994018.

Davranche, A., G. Lefebvre, and B. Poulin. 2010. "Wetland Monitoring Using Classification Trees and SPOT-5 Seasonal Time Series." Remote Sensing of Environment 114 (3):552-62. https://doi.org/10.1016/j.rse.2009.10.009. 
Dietterich, T. G. 1998. “Approximate Statistical Tests for Comparing Supervised Classification Learning Algorithms.” Neural Computation 10 (7):1895-1923. https://doi.org/10.1162/089976698300017197.

Duda, R. O., P. E. Hart, and D. G. Stork. 2001. Pattern Classification. Wiley.

Dymond, C. C., D. J. Mladenoff, and V. C. Radeloff. 2002. "Phenological Differences in Tasseled Cap Indices Improve Deciduous Forest Classification.” Remote Sensing of Environment 80 (3):460-72.

Foody, G. M. 2009. “Classification Accuracy Comparison: Hypothesis Tests and the Use of Confidence Intervals in Evaluations of Difference, Equivalence and NonInferiority." Remote Sensing of Environment 113 (8):1658-63. https://doi.org/10.1016/j.rse.2009.03.014.

Foody, G. M., and M. K. Arora. 1997. "An Evaluation of Some Factors Affecting the Accuracy of Classification by an Artificial Neural Network." International Journal of Remote Sensing 18 (4):799-810. https://doi.org/10.1080/014311697218764.

Foody, G. M., and A. Mathur. 2004. "A Relative Evaluation of Multiclass Image Classification by Support Vector Machines.” IEEE Transactions on Geoscience and Remote Sensing 42 (6):1335-43.

Foody, G. M., M. B. McCulloch, and W. B. Yates. 1995. "The Effect of Training Set Size and Composition on Artificial Neural Network Classification." International Journal of Remote Sensing 16 (9):1707-23. https://doi.org/10.1080/01431169508954507.

Friedl, M. A., and C. E. Brodley. 1997. "Decision Tree Classification of Land Cover from Remotely Sensed Data.” Remote Sensing of Environment 61 (3):399-409. https://doi.org/10.1016/S0034-4257(97)00049-7.

Gann, D., and J. H. Richards. 2013. "Evaluating High-Resolution Aerial Photography Acquired by Unmanned Aerial Systems for Use in Mapping Everglades Wetland Plant Associations.” Jacksonville, FL: U.S. Army Corps of Engineers. http://digitalcommons.fiu.edu/gis/27.

Gann, D., J. H. Richards, S. Lee, and E. Gaiser. 2015. “Detecting Calcareous Periphyton Mats in the Greater Everglades Using Passive Remote Sensing Methods." In Microbiology of the Everglades Ecosystem, edited by J. A. Entry, A. D. Gottlieb, K. J., and A. Ogram, 350-72. CRC Press. https://doi.org/doi:10.1201/b18253-17. 
Gao, T., J. Zhu, X. Zheng, G. Shang, L. Huang, and S. Wu. 2015. "Mapping Spatial Distribution of Larch Plantations from Multi-Seasonal Landsat-8 OLI Imagery and Multi-Scale Textures Using Random Forests.” Remote Sensing 7 (2):170220. https://doi.org/10.3390/rs70201702.

Gao, Z. G., and L. Q. Zhang. 2006. "Multi-Seasonal Spectral Characteristics Analysis of Coastal Salt Marsh Vegetation in Shanghai, China." Estuarine, Coastal and Shelf Science 69 (1-2):217-24. https://doi.org/10.1016/j.ecss.2006.04.016.

Gilmore, M. S., E. H. Wilson, N. Barrett, D. L. Civco, S. Prisloe, J. D. Hurd, and C. Chadwick. 2008. "Integrating Multi-Temporal Spectral and Structural Information to Map Wetland Vegetation in a Lower Connecticut River Tidal Marsh.” Remote Sensing of Environment 112 (11):4048-60. https://doi.org/10.1016/j.rse.2008.05.020.

Gislason, P. O., J. A. Benediktsson, and J. R. Sveinsson. 2006. "Random Forests for Land Cover Classification.” Pattern Recognition Letters 27 (4):294-300. https://doi.org/10.1016/j.patrec.2005.08.011.

Guisan, A., and N. E. Zimmermann. 2000. "Predictive Habitat Distribution Models in Ecology." Ecological Modelling 135 (2-3):147-86.

Heuvelink, G. B. M. 2002. "Analysing Uncertainty Propagation in GIS: Why Is It Not That Simple?" In Uncertainty in Remote Sensing and GIS, 155-65. John Wiley \& Sons, Ltd. https://doi.org/10.1002/0470035269.ch10.

Hijmans, R. J., and J. van Etten. 2010. "Raster: Geographic Analysis and Modeling with Raster Data". R Package Version 2.6. https://cran.rproject.org/web/packages/raster/index.html.

Ho, T. K. 1998. "The Random Subspace Method for Constructing Decision Forests." IEEE Transactions on Pattern Analysis and Machine Intelligence 20 (8):832-44. https://doi.org/10.1109/34.709601.

Hodges, J. L., and E. L. Lehmann. 1963. "Estimates of Location Based on Rank Tests." The Annals of Mathematical Statistics 34 (2):598-611. https://doi.org/10.1214/aoms/1177704172.

Huang, C., L. S. Davis, and J. R. G. Townshend. 2002. "An Assessment of Support Vector Machines for Land Cover Classification." International Journal of Remote Sensing 23 (4):725-49. https://doi.org/10.1080/01431160110040323. 
Jain, A. K., and B. Chandrasekaran. 1982. "Dimensionality and Sample Size Considerations in Pattern Recognition Practice." In Handbook of Statistics, 2:835-55. Elsevier. https://doi.org/10.1016/S0169-7161(82)02042-2.

Jakomulska, A. M., and J. P. Radomski. 2002. "Uncertainty in Land Cover Mapping from Remotely Sensed Data Using Textural Algorithm and Artificial Neural Networks." In Uncertainty in Remote Sensing and GIS, 99-118. John Wiley \& Sons, Ltd. https://doi.org/10.1002/0470035269.ch7.

Jensen, J. R. 2005. Introductory Digital Image Processing: A Remote Sensing Perspective. Edited by K. C. Clarke. Prentice Hall Series in Geographic Information Science. 3rd ed. Upper Saddle River, NJ 07459: Prentice Hall.

Jones, J. W. 2011. "Remote Sensing of Vegetation Pattern and Condition to Monitor Changes in Everglades Biogeochemistry." Critical Reviews in Environmental Science and Technology 41 (supp1):64-91.

Jones, J. W., G. B. Desmond, and C. Henkle. 2012. "An Approach to Regional Wetland Digital Elevation Model Development Using a Differential Global Positioning System and a Custom-Built Helicopter-Based Surveying System." International Journal of Remote Sensing 33 (November 2011):37-41.

Kanal, L., and B. Chandrasekaran. 1971. "On Dimensionality and Sample Size in Statistical Pattern Classification." Pattern Recognition 3 (3):225-34. https://doi.org/10.1016/0031-3203(71)90013-6.

Kim, J.-W., Z. Lu, J. W. Jones, C. K. Shum, H. Lee, and Y. Jia. 2014. "Monitoring Everglades Freshwater Marsh Water Level Using L-Band Synthetic Aperture Radar Backscatter." Remote Sensing of Environment 150:66-81. https://doi.org/10.1016/j.rse.2014.03.031.

Kuhn, M., Contributins from J. Wing, S. Weston, A. Williams, C. Keefer, A. Engelhardt, T. Cooper, et al. 2016. "Caret: Classification and Regression Training". R package Version 6.0. https://cran.r-project.org/web/packages/caret/index.html.

Kyriakidis, P. C., and J. L. Dungan. 2001. "A Geostatistical Approach for Mapping Thematic Classification Accuracy and Evaluating the Impact of Inaccurate Spatial Data on Ecological Model Predictions." Environmental and Ecological Statistics $8(4): 311-30$.

Langford, W. T., S. E. Gergel, T. G. Dietterich, and W. Cohen. 2006. “Map Misclassification Can Cause Large Errors in Landscape Pattern Indices: Examples from Habitat Fragmentation." Ecosystems 9 (3):474-88. 
Li, M., S. Zang, B. Zhang, S. Li, and C. Wu. 2014. "A Review of Remote Sensing Image Classification Techniques: The Role of Spatio-Contextual Information."

European Journal of Remote Sensing 47:389-411. https://doi.org/doi: 10.5721/EuJRS20144723.

Lunetta, R. S., R. G. Congalton, L. K. Fenstermaker, K. C. McGwire, and L. R. Tinney. 1991. "Remote Sensing and Geographic Information System Data Integration: Error Sources and Research Issues." Photogrammetric Engineering and Remote Sensing 57 (6):677-87.

Miller, D. M., E. J. Kaminsky, and S. Rana. 1995. "Neural Network Classification of Remote-Sensing Data." Computers \& Geosciences 21 (3):377-86. https://doi.org/10.1016/0098-3004(94)00082-6.

Mitscherlich, E. A. 1909. "The Law of the Minimum and the Law of Diminishing Soil Productivity (In German).” Landwirtschaftliche Jahrbücher 38:537-52.

National Research Council. 2014. Progress Toward Restoring the Everglades: The Fifth Biennial Review: 2014. Washington, DC: The National Academies Press. https://doi.org/10.17226/18809.

Ozesmi, S. L., and M. E. Bauer. 2002. "Satellite Remote Sensing of Wetlands.” Wetlands Ecology and Management 10 (5):381-402. https://doi.org/10.1023/a:1020908432489.

Pal, M. 2005. "Random Forest Classifier for Remote Sensing Classification." International Journal of Remote Sensing 26 (1):217-22. https://doi.org/10.1080/01431160412331269698.

Pal, M., and P. M. Mather. 2005. "Support Vector Machines for Classification in Remote Sensing.” International Journal of Remote Sensing 26 (5):1007-11. https://doi.org/10.1080/01431160512331314083.

Poulin, B., A. Davranche, and G. Lefebvre. 2010. "Ecological Assessment of Phragmites Australis Wetlands Using Multi-Season SPOT-5 Scenes.” Remote Sensing of Environment 114 (7):1602-9. https://doi.org/10.1016/j.rse.2010.02.014.

R Core Team. 2013. "R: A Language and Environment for Statistical Computing”. R Foundation for Statistical Computing. Vienna, Austria.

Revolution Analytics, and Steve Weston. 2013. "Foreach: Foreach Looping Construct for R.” R package Version 1.4.3. http://cran.r-project.org/package=foreach. 
Rodriguez-Galiano, V. F., M. Chica-Olmo, F. Abarca-Hernandez, P. M. Atkinson, and C. Jeganathan. 2012. "Random Forest Classification of Mediterranean Land Cover Using Multi-Seasonal Imagery and Multi-Seasonal Texture." Remote Sensing of Environment 121 (June):93-107. https://doi.org/10.1016/j.rse.2011.12.003.

Rogan, J., J. Franklin, D. Stow, J. Miller, C. Woodcock, and D. Roberts. 2008. "Mapping Land-Cover Modifications over Large Areas: A Comparison of Machine Learning Algorithms." Remote Sensing of Environment 112 (5):2272-83. https://doi.org/10.1016/j.rse.2007.10.004.

Sakamoto, T., M. Yokozawa, H. Toritani, M. Shibayama, N. Ishitsuka, and H. Ohno. 2005. "A Crop Phenology Detection Method Using Time-Series MODIS Data." Remote Sensing of Environment 96 (3-4):366-74. https://doi.org/10.1016/j.rse.2005.03.008.

Shao, Y., and R. S. Lunetta. 2012. "Comparison of Support Vector Machine, Neural Network, and CART Algorithms for the Land-Cover Classification Using Limited Training Data Points." ISPRS Journal of Photogrammetry and Remote Sensing 70:78-87. https://doi.org/10.1016/j.isprsjprs.2012.04.001.

Sklar, F. H., H. C. Fitz, Y. Wu, R. Van Zee, and C. McVoy. 2001. "South Florida: The Reality of Change and the Prospects for Sustainability: The Design of Ecological Landscape Models for Everglades Restoration." Ecological Economics 37 (3):379-401. https://doi.org/http://dx.doi.org/10.1016/S0921-8009(01)00180-X.

Smits, P. C., S. G. Dellepiane, and R. A. Schowengerdt. 1999. "Quality Assessment of Image Classification Algorithms for Land-Cover Mapping: A Review and a Proposal for a Cost-Based Approach." International Journal of Remote Sensing 20 (8):1461-86. https://doi.org/10.1080/014311699212560.

Szantoi, Z., F. J. Escobedo, A. Abd-Elrahman, L. Pearlstine, B. Dewitt, and S. Smith. 2015. "Classifying Spatially Heterogeneous Wetland Communities Using Machine Learning Algorithms and Spectral and Textural Features."

Environmental Monitoring and Assessment 187 (5):1-15. https://doi.org/10.1007/s10661-015-4426-5.

Van Niel, T., T. Mc Vicar, and B. Datt. 2005. "On the Relationship between Training Sample Size and Data Dimensionality: Monte Carlo Analysis of Broadband Multi-Temporal Classification." Remote Sensing of Environment 98 (4):468-80. https://doi.org/10.1016/j.rse.2005.08.011.

Wang, G. X., G. Z. Gertner, S. F. Fang, and A. B. Anderson. 2005. "A Methodology for Spatial Uncertainty Analysis of Remote Sensing and GIS Products." Photogrammetric Engineering and Remote Sensing 71 (12):1423-32. 
Weihs, C., U. Ligges, K. Luebke, and N. Raabe. 2005. "KlaR Analyzing German Business Cycles." In Data Analysis and Decision Support, edited by D. Baier, R. Decker, and L. Schmidt-Thieme, 335-43. Berlin: Springer-Verlag.

Wilcoxon, F. 1945. "Individual Comparisons by Ranking Methods." Biometrics Bulletin 1 (6):80-83. https://doi.org/10.2307/3001968.

Xhemali, D., C. J. Hind, and R. G. Stone. 2009. "Naïve Bayes vs. Decision Trees vs. Neural Networks in the Classification of Training Web Pages." International Journal of Computer Science Issues 4 (1):16-23.

Zweig, C. L., M. A. Burgess, H. F. Percival, and W. M. Kitchens. 2015. "Use of Unmanned Aircraft Systems to Delineate Fine-Scale Wetland Vegetation Communities." Wetlands 35 (2):303-9. https://doi.org/10.1007/s13157-014-06124.

Zweig, C. L., and W. M. Kitchens. 2009. "Multi-State Succession in Wetlands: A Novel Use of State and Transition Models." Ecology 90 (7):1900-1909. 


\section{CONCLUSIONS}

When aggregating data, information is generalized and, therefore, information is lost. Generalization of fine-scale data is often necessary to support coarser-scale modeling efforts, but the optimal degree of generalization is subjective. Validity of generalized data is application-specific, and the scientific question posed by the researcher is ultimately the deciding factor in determining the data's adequacy. In the context of categorical data scaling, two thresholds are of interest: the minimum level of thematic class precision that is required to maintain enough information to answer the scientific question; and the threshold for a class's representativeness, beyond which it is of no ecological interest at the aggregated scale. The minimum level of class precision is the point beyond which generalization reduces the information content to levels where the question of interest can no longer be adequately addressed. Both parameters, precision and representativeness, need to be determined with respect to ecological validity and significance. The purpose of this study was to develop a new scaling algorithm that addresses the problem of uncontrolled information loss and allows the data analyst or modeler to conduct sensitivity analysis for the effects of precision and representativeness on modeling results.

The MDGP-scaling algorithm proposed in Chapter 1 was developed in the context of landscape ecology and integrates concepts of community ecology and phytosociology, acknowledging variability in co-occurrence patterns of species or community classes as spatial scales change (i.e., cell size increases). The algorithm overcomes the limitations of methods for quantitative grouping often employed in the fields of community ecology and phytosociology, such as cluster algorithms. The 
proposed criteria for evaluation of algorithm consistency were location-specific information retention integrated across the landscape, scaled class count consistency, and class-label fidelity. An information-retention metric was introduced as an effective metric to compare agreement of categorical data vectors; this metric can be used to evaluate information loss and to classify samples to a quantitatively-defined classification system. Information agreement is a true metric and, hence, is a valid evaluation parameter that facilitates direct comparison of scaling results between algorithms or between different parameter settings. The results of this research strongly supports the application of the newly developed algorithm to scale categorical landscape representations to lower (coarser) spatial resolutions.

The simulation study in Chapter 1 was conducted to establish confidence in the scaling properties of the algorithm and the applicability across a large range of landscape settings and categorical data scaling needs. The algorithm was robust in consistently generating representative class labels while significantly increasing information retention for the scaled landscapes when compared to other commonly used algorithms. Low variability in class-count ratios and class-label fidelity provide the foundation for confidence in reproducibility and reliability of the MDGP-scaling algorithm.

Simulation results suggest that, especially when dealing with less aggregated, patchy landscapes, the $M D G P$-scaling algorithm was very successful in retaining information at a high level when other algorithms (i.e., majority and random rule) failed to do so. Information loss for an aggregated spatial unit of a scaled map increased drastically with richness of the original landscape and with scale factor. Implementing a scaling parameter that controlled class-label precision effectively reduced information 
loss of scaled landscapes when class-label precision was increased. Results were consistent for all evaluated landscapes and scale factors.

The MDGP-scaling algorithm is the first algorithm that generates data-driven scale-appropriate classification schemes while conducting spatial data aggregation. This study demonstrated that the algorithm consistently delivers representative class descriptors (labels), generating new, scale-specific classification systems. To attain adequate precision in the thematic domain that supports the use of the aggregated product in subsequent ecological models, the algorithm provides a control parameter that allows for optimization of information retention and label fidelity in the thematic domain.

The secondary objectives of this study were to (1) evaluate the effects of classlabel precision on class-label fidelity and information retention when scaling highprecision vegetation maps of real-world landscapes using the $M D G P$-scaling algorithm, (2) to determine optimal class-label precision and class representativeness thresholds and (3) to evaluate the correlation of class-label precision and representativeness of scaled classes to their detectability from remotely sensed data of lower spatial resolution. In Chapter 2, results of the scaling analysis for two natural wetlands landscapes indicate that optimizing class-precision parameters and representativeness is possible. Differences in information retention for optimal scaling solutions were significantly higher than the trivial majority-rule solutions, regardless of landscape. Precise and representative classes were detectable from lower resolution remotely sensed data with acceptable accuracy, and that accuracy increased when compared to the standard majority-rule method. 
The trade-offs of information retention, label fidelity, and spectral detectability of scaled classes from multi-spectral data indicate that there is not a single best solution. Weighting these criteria when selecting the optimal solution is user- and applicationdependent. Class-specific accuracy and classification scheme preferences can be used in the selection of the optimal parameter selection. The method presented provides a framework that integrates the quantitative evaluation of scaling parameter selection and its effects on representativeness of classification systems, information retention at the local (pixel) and at the landscape level, and for the spectral detection probabilities of the scaled classes. Applying this method allows for user-specific and preference optimized solutions where previously no weighting of effects was possible.

Since scaling of high-resolution categorical maps relies on accurate representations of the landscape, the effects of classification methods on classification accuracy and method efficiency were assessed in Chapter 3. Trade-offs exist for training sample size and feature-space dimensionality on overall accuracy, location-specific classification confidence, class-specific accuracies and classifier training and prediction times. A framework to model the interactions of training sample intensity, feature space, and their effects on mapping accuracies and processing efficiency was developed. This framework was then used to develop and optimize a mapping method for wetland plant communities from high resolution multi-spectral satellite data. Evaluating and selecting mapping methods that promise high accuracies at high spatial resolution (precision) while keeping production costs low is especially important when mapping large spatial extents at multiple time-intervals. Many monitoring programs rely on high precision class detection and mapping products. Results of this study show that the comparison of 
mapping methods on the basis of overall accuracy, confidence estimates, and classifier efficiency allows for optimization of mapping methods.

The results from Chapter 3 indicate that increase in sampling intensity boosts accuracies in a reasonably predictable fashion. However, the addition of a second image acquired at a time when ground conditions and phenological differences in vegetation types are observed had a much higher impact on classification accuracy, increasing accuracy even at very low sampling intensities to levels that were never reached with a single season image. If bi-seasonal data acquisition is not possible, knowing which timeframe maximizes single season imagery is crucial as the second-best option. In this study, wet-season data provided this second-best option, but the analysis also showed that some classes benefit more from dry-season and others from wet-season data, which emphasizes the need to consult class-specific user's and producer's accuracies when specific classes are of higher priority than others. Selecting data on the basis of overall accuracy alone does not provide enough information in these cases. Vegetation that was consistent or stable between the two seasons (same class label) benefitted from the use of a bi-seasonal dataset. 
VITA

\section{DANIEL GANN}

Born, Leonberg, Germany

1992 - 1998 1.Staatsexamen Pedagogy; Education (Biology, Physical Education, ESL) Pädagogische Hochschule, Ludwigsburg Germany.

2000 - $2003 \quad$ M.S. Environmental Sciences, Florida International University, Miami, FL.

2005-2018 Adjunct Professor, Department of Earth and Environment and Department of Biological Sciences, Florida International University, Miami, FL.

2005 - 2018 Research Associate, GIS-RS Center, Florida International University, Miami, FL.

2012 - 2018 Doctoral Candidate, Florida International University, Miami, FL.

\section{PUBLICATIONS}

Gann, D. 2014. Remote Sensing Supported Vegetation Detection in the Hole-in-theDonut Restoration Areas. Everglades National Park, Florida City, FL. Report. 31 pgs.

Gann, D. \& J. H. Richards. 2015. Quantitative Comparison of Plant Community Hydrology Using Large-Extent, Long-Term Data. Wetlands, 35(1), 81-93. http://doi.org/10.1007/s13157-014-0594-2

Gann, D., J.H. Richards, and H. Biswas. 2012. Determine the Effectiveness of Vegetation Classification Using WorldView 2 Satellite Data for the Greater Everglades. South Florida Water Management District, West Palm Beach, FL. Report. 62 pgs.

Gann, D., J. H. Richards, and H. Biswas. 2013. Evaluating accuracy improvements in wetland vegetation classification at two scales using bi-seasonal remotely sensed data. MultiTemp 2013. Banff, Alberta, Canada. Oral.

Gann, D., J. H. Richards, and A. Gottlieb. 2012. Evaluation of WorldView-2 and Landsat data to differentiate and map freshwater marsh plant communities at different spatial scales for two Everglades landscapes. 9th INTECOL International Wetlands Conference 2012. Orlando, FL., USA. Oral. 
Gann, D., J. H. Richards, S. Lee, E. Gaiser. 2015. Detecting Calcareous Periphyton Mats in the Greater Everglades Using Passive Remote Sensing Methods. In J.

A. Entry, A. D. Gottlieb, K. Jayachandran, \& A. Ogram (Eds.), Microbiology of the Everglades Ecosystem, 350-372. CRC Press. http://doi.org/doi:10.1201/b18253-17

Gann, D, J. H. Richards \& P. Olivas. 2016. Monitoring vegetation change patterns in coastal wetlands under sea-level rise - a multi-scale remote sensing approach. Ecological Society of America Annual meeting, Ft. Lauderdale, FL., August 2016. Oral.

Gann, D., J. H. Richards, J. Sadle. 2015. Detecting vegetation change in northern Shark River Slough from remotely-sensed data. Greater Everglades Ecosystem Restoration (GEER). Coral Springs, FL., USA, Apr. 23, 2015. Oral.

Mango, L. M., A. M. Melesse, M. E. McClain, D. Gann, S. G. Setegn. 2010. A modeling approach to determine the impacts of land use and climate change scenarios on the water flux of the upper Mara River. Hydrol. Earth Syst. Sci. Discuss., 7(4), 5851-5893. http://doi.org/10.5194/hessd-7-5851-2010

Mango, L. M., A. M. Melesse, M. E. McClain, D. Gann, S. G. Setegn. 2011. Land use and climate change impacts on the hydrology of the upper Mara River Basin, Kenya: results of a modeling study to support better resource management. Hydrology and Earth System Sciences, 15(7), 2245-2258. http://doi.org/10.5194/hess-15-2245-2011

Richards, J. H. \& D. Gann. 2015. Vegetation Trends in Indicator Regions of Everglades National Park. Everglades National Park, Florida City, FL. Report. 171 pgs.

Richards, J. H., D. Gann, L. Scinto, Y. Cai, and G. Liu. 2017. Environmental Monitoring and Assessment Program (REMAP) IV: Greater Everglades Whole-Ecosystem Monitoring and Assessment Final Report. 208 pgs.

Wendelberger, K., Gann, D., \& Richards, J. H. 2018. Using Bi-Seasonal WorldView-2 Multi-Spectral Data and Supervised Random Forest Classification to Map Coastal Plant Communities in Everglades National Park. Sensors, 18(3). https://doi.org/10.3390/s18030829

Zhang, K., B. Thapa, M. Ross, D. Gann. 2016. Remote Sensing of Seasonal Changes and Disturbance in Mangrove Forest: A Case Study from South Florida. Ecosphere 7(6). http://doi.org/10.1002/ecs2.1366 